Universidade de São Paulo

Instituto de Física

\title{
Robustez do Emaranhamento em Variáveis Contínuas e Fotodetecção de Feixes Intensos no Domínio Espectral
}

\author{
Felippe Alexandre Silva Barbosa
}

Orientador: Prof. Dr. Marcelo Martnelli

Tese de doutorado apresentada ao Instituto de Física para a obtenção do título de Doutor em Ciências

Banca Examinadora:

Carlos Henrique Monken (UFMG) Almir Ordacgi Caldeira (UNICAMP)

Antônio Fernando Ribeiro de Toledo Piza (USP)

Paulo Teotônio Sobrinho (USP)

Marcelo Martinelli (USP) 
FICHA CATALOGRÁFICA

Preparada pelo Serviço de Biblioteca e Informação do Instituto de Física da Universidade de São Paulo

Barbosa, Felippe Alexandre Silva

Robustez do emaranhamento em variáveis contínuas e fotodetecção de feixes intensos no domínio espectral. São Paulo, 2013.

Tese (Doutorado) - Universidade de São Paulo.

Instituto de Física, Departamento de Física Experimental.

Orientador: Prof. Dr. Marcelo Martinelli

Área de Concentração: Ótica quântica

Unitermos: 1. Óptica quântica; 2. Informação quântica; 3. Óptica não linear.

USP/IF/SBI-068/2013 


\section{Resumo}

Investigamos o emaranhamento gerado pelo oscilador paramétrico ótico (OPO) operando acima do limiar. Mostramos que o sistema tripartite, preparado diretamente usando o OPO e formado pelos feixes gêmeos e pelo campo de bombeio refletido, é inseparável. Esta foi a primeira observação de emaranhamento entre três frequências distintas de luz. Também investigamos a dinâmica deste emaranhamento sob a ação de perdas por transmissão. Dependendo da região de potência do campo de bombeio, pudemos preparar estados tripartites cujo emaranhamento inicial entre bombeio e feixes gêmeos se mantém ou é perdido após a atenuação dos mesmos. Estendemos o estudo para o sistema bipartite formado pelos feixes gêmeos. Neste sistema mais simples, pudemos investigar em detalhes a robustez do emaranhamento e as condições nas quais o emaranhamento se torna frágil. Também conduzimos um estudo teórico e derivamos uma condição suficiente para que um estado emaranhado formado por dois modos seja robusto frente a perdas. Esta condição se torna necessária para estados gaussianos e nos permite traçar uma fronteira no espaço de estados emaranhados que separa os estados robustos dos frágeis.

Estudamos em mais detalhes o processo de medição de flutuações de intensidades no domínio da frequência. As flutuações na intensidade são convertidas para frequências de rádio, com banda limitada pela detecção. Isto se reflete no fato de que cada componente de frequência $\Omega$ da fotocorrente contém a informação referente a dois modos do campo eletromagnético, as chamadas bandas laterais. Isto faz com que cada campo de luz que descrevemos seja de fato formado por dois modos, fazendo o nosso sistema mais rico. Demonstramos que o sistema hexapartite gerado pelo OPO, e formado pelas duas bandas laterais de cada um dos campos, é inseparável. Também descrevemos as condições dentro das quais a aproximação de um modo por campo, utilizada nos nossos outros trabalhos e por quase toda a comunidade, é válida. Mostramos também que a deteç̧ão com cavidades é capaz de caracterizar completamente o sistema gaussiano formado pelas duas bandas laterais de cada campo e que a deteç̧ão homodina, o método mais usado para caracterizar o estado quântico em variáveis contínuas, falha em medir todas as correlações presentes no sistema bimodal. 



\section{Abstract}

We investigate the entanglement generated by an optical parametric oscillator (OPO) operating above threshold. We showed that the tripartite system, prepared directly from the OPO and composed by the twin beams and the reflected pump field, is completely inseparable. This was the first observation of entanglement between three different frequencies of light. We also investigated the dynamics of entanglement under transmission losses. Depending on the injected pump power, we were able to prepare states with tripartite entanglement between the initial pump and twin beams that was maintained or lost after transmission losses. We extended the study to the bipartite system formed by the twin beams. In this simpler system, we investigated in more detail the robustness of entanglement and the conditions under which the entanglement becomes fragile. We also conducted a theoretical study and derived a sufficient condition for the entangled state formed by two modes to be robust against losses. This condition also become necessary for Gaussian states.

We also studied the measurement process of intensity fluctuations in the frequency domain. The fluctuations are converted to the radiofrequency range, with bandwidth limited by the detector. This is also reflected in the fact that each frequency component, $\Omega$, of the photocurrent contains the information relative to the two modes of the electromagnetic field equally spaced from its center frequency, the sidebands. Therefore each light beam we describe is actually composed by two modes, making our system more rich. We were able to demonstrate that this hexapartite system generated by our OPO, and composed by the two sidebands of each field, is completely inseparable. We also described under which conditions the approximation of one mode per field, used in our prior work and for almost the entire community, is valid. We also showed that resonator detection is able to completely characterize the system composed by the two sidebands of each field if the state is Gaussian and that homodyne detection, the method used to characterize the quantum state for continuous variables, fails to measure all correlations present in this bimodal system. 



\section{Agradecimentos}

Em primeiro lugar agradeço ao Marcelo Martinelli pela orientação realizada durante esses últimos seis anos. A sua atuação no momento de solucionar questões do dia-a-dia do laboratório foi essencial para todos os resultados que obtivemos durante meu doutorado. Pude aprender muito com a sua maneira de abordar os problemas físicos.

Agradeço ao Paulo Nussenzveig também pela orientação e pelas interessantes discussões físicas, ainda que não tão frequentes. Pude aprender muito com ele e com a visão que tem da Física como um todo.

Agradeço aos membros da banca examinadora, Carlos Monken, Almir Caldeira, Antônio de Toledo Piza e Paulo Teotônio Sobrinho por terem aceitado participar da minha banca examinadora e pelas contribuições ao texto da tese.

Agradeço ao meu parceiro de experimento, Antonio Sales M. Coelho, por todas as experiências compartilhadas. Os resultados que apresento nesta tese não seriam possíveis sem a sua presença. Agradeço por tudo! Em especial pelo trabalho lado-alado e sempre com o compromisso de conseguir os melhores dados possíveis.

Agradeço ao Alessandro Villar e ao Alencar de Faria pela parceria nas partes teóricas dos nossos trabalhos. Grande parte do que aprendi durante o doutorado foi fruto das discussões que tive com ambos. Mesmo algumas não sendo muito agradáveis, as nossas conversas sempre me desafiaram a pensar e entender mais e melhor sobre o sistema físico que estudamos.

Agrdeço à Katiúscia Cassemiro pelo trabalho desenvolvido em conjunto. Agradeço também ao Claude Fabre e ao Julian Laurat por terem me recebido no LKB e me proporcionado a oportunidade de trabalhar em um laboratório com tamanha história dentro da Física.

Também extendo os meus mais calorosos agradecimentos a todos os outros amigos e colegas que passaram pelo LMCAL, em especial ao Paulo Valente, Rodrigo Alves de Lima, Clodoaldo da Silva, Hélio Zhang He, Hans Florez, Márcio Heraclyto, Luciado 
da Cruz, Laércio Benedito, Flávio de Moraes, Luís Xavier, Rayssa Bruzaca, Renato Domeneguetti, Paula Meirelles, Carlos Gonzales, Klara Theophilo, Igor Konieczniak, Roberto Tsukino, Rodrigo Thomas, Renné Medeiros e Ashok Kumar.

Agradeço a todos que me receberam muito bem no período que passei na França, em especial a Olivier Morin, Roman Schmeissn, Nicolas Treps, Clement, Jian Li, John Roslund e ao Renné Medeiros, por, além de tudo, ter me hospedado em sua casa no início da minha estadia em Paris.

Agradeço aos demais professores que participaram da minha formação e que me influenciaram de maneira positiva. Em especial agradeço a Ademir Santana, Tarcísio Marciano, Paulo Caldas, Wadih Maluf, Joaquim Neto, Otil Lara, Nigel Pitt, Renata Funchal, Sílvio Salinas e Josif Frenkel. Agradeço também às funcionárias da secretaria da FEP - Edi e Juliane - e aos da oficina da Mecânica Central e da FEP - Wilson, Marcos, Donato e José Carlos - pelo seu trabalho sério e competente e por estarem sempre dispostos a me ajudar. Agradeço também a todos os maus professores e aos malditos burocratas que tive o desprazer de encontrar durante a minha vida e que deixarei inominados, pois não merecem espaço distinto na minha memória. Com eles também pude aprender uma lição muito importante: o que, definitivamente, não fazer!

Agradeço ao Jonatas, João, Álvaro e Patriota pelas infindáveis e interessantes conversas sobre todo e qualquer assunto, às vezes física, matemática, filosofia, política, às vezes esportes, vídeo games e diversas outras bobagens! Tenho também um agradecimento especial a fazer ao Alexandre Patriota pelas interessantes e produtivas discussões sobre os métodos estatísticos utilizados nesta tese.

Agradeço à minha namorada Ana Carolina pela parceria e companheirismo, pelo apoio e pelos momentos maravilhosos que vivemos juntos. Ter você ao meu lado, me faz mais feliz a cada dia! Agradeço também a sua família, em especial aos seus pais, José e Vera.

Meus agradecimentos mais importantes são reservados aos meus pais, Maria Ester e Simplício, aos meus irmãos, Túlio e Ícaro, e a todos os meus familiares e amigos que, por serem muitos, não vou nomeá-los por medo de cometer alguma injustiça. Cada um ajudou a moldar a minha personalidade e a vocês devo tudo o que eu sou!

E por último, agradeço à FAPESP pelo fomento. 
Toda mudança é uma ameaça à estabilidade. Essa é outra razão que nos torna pouco propensos a utilizar invenções novas. Toda descoberta da ciência pura é potencialmente subversiva: até a ciência deve, às vezes, ser tratada como um inimigo possível. Sim, a própria ciência.

- Ciência? O Selvagem franziu a testa. Conhecia a palavra. O que significava exatamente, porém, ele não o sabia. Shakespeare e os velhos do pueblo nunca se haviam referido à ciência ... Fez um esforço desesperado para compreender o que o Administrador queria dizer.

- Sim - continuou Mustafá Mond - essa é outra parcela no custo da estabilidade. Não é somente a arte que é incompatível com a felicidade, também o é a ciência. Ela é perigosa; temos de mantê-la cuidadosamente acorrentada e amordaçada.

- O quê? - exclamou Helmholtz, assombrado. - Mas nós vivemos repetindo que a ciência é tudo ... E toda a propaganda da ciência que fazemos no Colégio.

- Sim, mas que espécie de ciência? - perguntou sarcasticamente Mustafá Mond. - Os senhores não receberam instrução científica, de modo que não têm condições de julgar. Quanto a mim, fui um bom físico, no meu tempo. Bom demais, bastante bom para compreender que toda a nossa ciência é simplesmente um livro de cozinha, com uma teoria ortodoxa de arte culinária que ninguém tem o direito de contestar ... Pus-me a cozinhar um pouco a meu modo. Cozinha heterodoxa, cozinha ilícita. Um pouco de ciência verdadeira, em suma. Calou-se.

- E que aconteceu? - perguntou Helmholtz Watson.

O Adminsitrador suspirou. - Quase aconteceu o mesmo que vai acontecer aos senhores, meus jovens amigos. Estive a ponto de ser mandado para uma ilha.

Aldous Huxley - Admirável Mundo Novo 

Dedicada a todos que procuram fazer deste mundo um lugar melhor 



\section{Sumário}

\begin{tabular}{llr}
\hline 1 & Introdução & p. 16
\end{tabular}

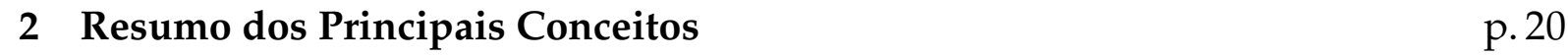

$2.1 \quad$ Emaranhamento e Desigualdades de Bell $\ldots \ldots \ldots$. . . . . . . . p. 20

2.2 Paradoxo EPR . . . . . . . . . . . . . . . . . p. 23

2.3 Oscilador Paramétrico Ótico e Conversão Paramétrica Descendente . . p.26

2.4 Cavidades Óticas $\ldots \ldots \ldots \ldots$. . . . . . . . . . . . . . . . . . . . . . . . 29

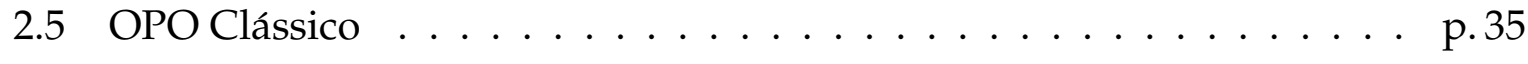

3 Métodos de Medida das Flutuações da Luz p.43

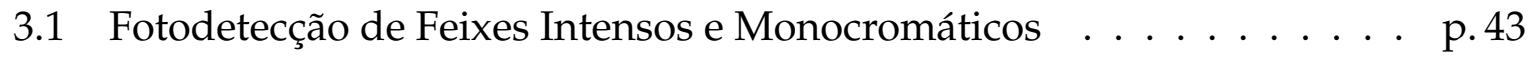

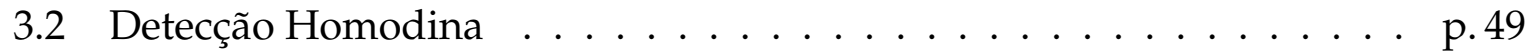

3.3 Limite Quântico Padrão - Shot Noise . . . . . . . . . . . . . . . . . . p.51

3.4 Medidas no Domínio da Frequência - Demodulação . . . . . . . . . . . p.53

3.4.1 Teorema de Wiener-Khinchin e Fotocorrente Estacionária . . . . p. p.53

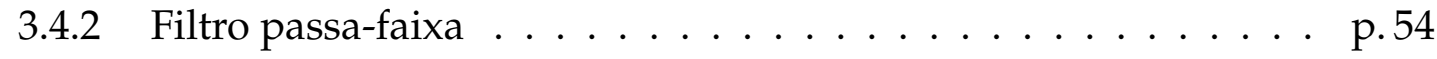

3.4 .3 Demodulação . . . . . . . . . . . . . . . . p. 57

3.4 .4 Dupla Demodulação . . . . . . . . . . . . . . . . p. 59

3.4 .5 Difusão de Fase $\ldots \ldots \ldots \ldots \ldots$. . . . . . . . . . . . . . . .

3.5 Detecção com Cavidade . . . . . . . . . . . . . . . . . p. 64

3.6 Generalização para Mais de um Campo . . . . . . . . . . . . . . . p.72

3.6 .1 Detecção Homodina . . . . . . . . . . . . . . . p. 72 
3.6 .2 Deteç̧ão com Cavidades $\ldots \ldots \ldots \ldots \ldots$. . . . . . . p.74

3.6.3 Relações entre Operadores Espectrais e das Bandas Laterais . . p.77

\begin{tabular}{lll}
\hline & Descrição do Aparato Experimental & p. 80
\end{tabular}

$4.1 \quad$ Laser de Bombeio, OPO e Cavidade de Filtro $\ldots \ldots \ldots \ldots$. . . . p. 81

4.2 Detetores e Filtros Eletrônicos $\ldots \ldots \ldots \ldots \ldots \ldots \ldots$ p. . . . . . . .

4.3 Cadeia de Demodulação e Aquisição $\ldots \ldots \ldots \ldots \ldots$. . . . . . . 85

4.4 Calibração do Shot Noise $\ldots \ldots \ldots \ldots \ldots \ldots$. . . . . . . . . 87

4.5 Estabilização de Frequência e Temperatura $\ldots \ldots \ldots \ldots$. . . . . p.90

5 Emaranhamento em Variáveis Contínuas $\quad$ p.95

$5.1 \quad$ Emaranhamento $\ldots \ldots \ldots \ldots \ldots \ldots \ldots \ldots \ldots \ldots \ldots$

5.2 Variáveis Contínuas $\ldots \ldots \ldots \ldots \ldots \ldots \ldots \ldots \ldots \ldots$

5.3 Transformações Simpléticas e PPT em Variáveis Contínuas . . . . . . p p.102

5.3 .1 Transformações Simpléticas . . . . . . . . . . . . . . . . . . p. p. 103

5.3.2 Autovalores Simpléticos e Teorema de Williamson. . . . . . . p p.105

5.3 .3 Sistemas de Dois Modos . . . . . . . . . . . . . . . p. p. 106

5.4 Análise da Robustez do Emaranhamento em Sistemas de Variáveis

Contínuas de Dois Modos . . . . . . . . . . . . . . . . . . p.111

5.4 .1 Critério PPT após Atenuação … . . . . . . . . . . . p. p.111

5.4 .2 Critérios para a Robustez do Emaranhamento . . . . . . . . p.115

$5.4 .3 \quad$ Exemplos de Estados Simples. . . . . . . . . . . . . . . . . p. 119

5.4 .4 Comentário Sobre Estados Não-Gaussianos . . . . . . . . . . . p.123

6 Descrição Teórica do OPO Triplamente Ressonante $\quad$ p.125

6.1 Descrição Quântica dos Processos Físicos Envolvidos … . . . . . . p. 125

6.2 Análise Espectral do OPO . . . . . . . . . . . . . . . . . . . . . p. 129

6.2 .1 Função de Wigner e Equações de Langevin . . . . . . . . . . . . p.129 
6.2 .2 Solução Espectral para as Flutuações. . . . . . . . . . . . . . . p.131

6.2.3 Emaranhamento e Excesso de Ruído de Fase Introduzido pelos Fônons . . . . . . . . . . . . . . . . . . . . . p.135

$6.2 .4 \quad$ Squeezing de Dois Modos $\ldots \ldots \ldots \ldots$. . . . . . . p. . . . . .

6.3 Correlações EPR no OPO . . . . . . . . . . . . . . . . . p. 140

7 Medidas de Emaranhamento e Desemaranhamento no OPO p.142

$7.1 \quad$ Emaranhamento e Robustez no OPO $\ldots \ldots \ldots \ldots \ldots$. . . . . . . . 142

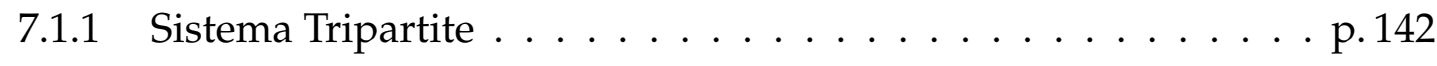

7.1.2 Sistema Bipartite formado pelos Feixes Gêmeos . . . . . . . . p. 146

7.2 Descrição do OPO nas Bandas Laterais. . . . . . . . . . . . . . . p. 148

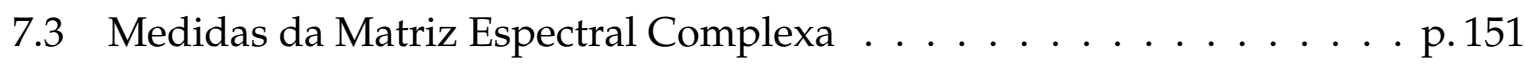

$7.3 .1 \quad$ Ajustes dos Ruídos e Correlações Espectrais … . . . . . . p. p.153

7.3 .2 Estimativa das Barras de Erro . . . . . . . . . . . . . p. 154

$7.4 \quad$ Emaranhamento Hexapartite no OPO $\ldots \ldots \ldots \ldots \ldots$. . . . . . . . 157

7.5 Comparação da Detecção Homodina com a Detecção com Cavidades · p.159

7.6 Discussão sobre Gaussianidade $\ldots$. . . . . . . . . . . . . . . p p. 161

7.6 .1 Teste de Hipóteses . . . . . . . . . . . . . . . . . . . . p.161

7.6 .2 Teste de Shapiro-Wilk $\ldots \ldots \ldots \ldots \ldots \ldots \ldots$. . . . . . . . . . . .

8 Conclusões e Perspectivas $\quad$ p. 168

$\begin{array}{ll}\text { Referências Bibliográficas } & \text { p. } 170\end{array}$ 


\section{Introdução}

O emaranhamento é uma propriedade presente somente em sistemas quânticos, pois não pode ser explicada por meio de uma teoria pobabilística clássica. Ele está associado a tipos especiais de correlação e foi tema de uma discussão entre Einstein, Bohr e Schrödinger durante os anos de 1935 e 1936. O emaranhamento possui, além do interesse fundamental por se tratar de uma propriedade intrinsecamente quântica, aplicações em diversos protocolos para processamento, armazenamento e comunicação de informação codificada em estados quânticos, assim como em protocolos de criptografia, que o utilizam para garantir a segurança da informação [1]. Dentre as aplicações de estados emaranhados estão o teletransporte [2, 3], a codificação densa [4] e a computação quântica com estados puros [5]. Os últimos anos tem presenciado um imenso esforço coletivo para implementação dos sistemas de processamento, comunicação e armazenamento de informação quântica, que tem como objetivo principal permitir a manipulação de estados quânticos, fazendo com que seja possível utilizá-los para codificar informação. Este tipo de programa possui diversas vertentes e grandes desafios em todas elas, como a construção de computadores quânticos [6, 7], a implementação de redes quânticas de processamento e armazenamento de informação [8, 9] e sistemas de criptografia quântica [10]. Estas implementações necessitam de diversos recursos, dentre eles o emaranhamento. Nosso trabalho se encaixa como uma peça neste esforço coletivo.

O principal objetivo neste trabalho foi estudar o emaranhamento em variáveis contínuas, continuando uma linha de pesquisa sobre emaranhamento no oscilador paramétrico ótico $(O P O)$ já em andamento no laboratório [11, 12, 13, 14, 15, 16, 17]. Para isto, preparamos estados emaranhados entre três feixes intensos de luz, um deles com comprimento de onda de $532 \mathrm{~nm}$ (bombeio) e os outros dois por volta de $1064 \mathrm{~nm}$ (feixes gêmeos). Utilizamos um $O P O$, que consiste de um cristal não-linear colocado dentro de uma cavidade ótica ressonante, para preparar os feixes emaranhados. $\mathrm{Ob}$ servamos o emaranhamento através de flutuações de fase e intensidade dos campos 
que deixam a cavidade. No contexto quântico, elas são descritas por operadores com a mesmas relações de comutação que a posição e o momento de uma partícula. Elas são chamadas de variáveis contínuas por possuírem espectro de autovalores contínuo, em contraste com observáveis cujos espaços de Hilbert possuem espectro discreto, como spins e níveis atômicos de energia [1]. Devido à presença de emaranhamento entre diferentes frequências, podemos utilizar os estados gerados pelo OPO para transportar um estado quântico entre frequências distintas do espectro eletromagnético por meio do teletransporte. O processo não-linear responsável pelo emaranhamento no OPO é a Conversão Paramétrica Descendente, que é caracterizada pela absorção de um fóton do bombeio e a emissão de dois outros cuja soma das energias se iguala à do primeiro. Os fótons gerados são realimentados pela cavidade fazendo com que ocorra ganho. Quando este ganho supera as perdas na cavidade o sistema oscila gerando outros dois feixes de luz, cuja soma das frequências é igual à do primeiro. Estes campos possuem fortes correlações de intensidade e fase, que são as responsáveis pelo emaranhamento.

Além da preparação, investigamos também a dinâmica deste emaranhamento frente a perdas por transmissão. É bem conhecido que o emaranhamento em geral é degradado por perdas, e isto constitui um problema de grande importância para a comunicação quântica e motivou diversas ideias para contorná-lo. Protocolos para concentrar (destilar) o emaranhamento em sistemas fracamente emaranhados [18] e transmiti-lo por longas distâncias com pouca degradação [19] foram propostos e implementados com sucesso para alguns sistemas [20, 21, 22, 23] . Entretanto, todos eles necessitam de algum emaranhamento, mesmo que pequeno, para serem bem sucedidos. Analisamos em que condições estados de variáveis contínuas, formado por dois modos, podem ter seu emaranhamento completamente perdido devido a perdas por transmissão. Estudamos este problema experimentalmente utilizando apenas os feixes gêmeos gerados pelo OPO, que estão emaranhados entre si. Os estados robustos com relação às perdas são os mais interessantes para aplicações em comunicação quântica, pois seu emaranhamento persiste até a atenuação total. Derivamos também uma quantidade que nos diz se o estado inicial é robusto e, em caso negativo, o quanto ele pode ser atenuado antes que seu emaranhamento seja completamente perdido.

Outra tarefa necessária para a implementação bem sucedida dos sistemas de informação quântica está ligada com a capacidade de caracterizar devidamente um estado quântico. Como temos acesso somente aos elementos diagonais do estado, é necessário realizar medidas em diversas bases para poder caracterizá-lo por completo. Procedimentos que implementam estas ideias ficaram conhecidos como tomografia de estados quânticos 
[24], por analogia com a tomografia em Medicina, e dentre eles se destaca a tomografia homodina [25]. Estes procedimentos são um conjunto de métodos estatísticos usados para extrair o estado quântico das medidas das probabilidades marginais. Entretanto, para que isto possa ser feito, o primeiro requisito é que a medida em questão seja sensível a toda informação relevante para que o estado quântico possa ser a estimado. Percebemos que a detecção homodina, peça chave na tomografia homodina, não satisfaz essa condição quando as medidas são realizadas diretamente no domínio da frequência mesmo para estados gaussianos. Por outro lado, o método para fazer a conversão de ruído de fase em ruído de intensidade usado nos últimos trabalhos do grupo [13, 16, 26, 27] é sensível a toda essa informação relevante no caso em que o estado é gaussiano. Trabalhar no domínio da frequência permite algumas vantagens em relação ao domínio do tempo. A principal delas é a possibilidade de aumento da capacidade de um canal de comunicação quântico. Da mesma forma que a informação clássica transmitida pelos sistemas de rádio e televisão é codificada em diversas frequências do campo eletromagnético, a informação quântica poderia ser codificada também em diversas frequências em uma espécie de multiplexação [28]. Mas para isto é necessário entender o processo de medição de informação quântica no domínio da frequência. Durante o meu doutorado também conduzimos investigações dentro desta temática.

O texto está organizado da seguinte forma. No capítulo 2, faço uma breve discussão sobre os conceitos mais importantes e que permeiam todo o resto do texto, que são o emaranhamento, cavidades óticas e o oscilador paramétrico ótico. Este capítulo serve como base para todos os restantes. No capítulo 3, descrevo teoricamente em detalhes o processo de medição que utilizamos. Nele é discutida a conversão da luz em corrente elétrica no fotodiodo e como este processo nos permite acessar as quadraturas do campo elétrico. Também discuto a medida no domínio da frequência e como a mesma é intrinsecamente bimodal nos modos das bandas laterais. Ao final, apresento contribuições originais sobre como a detecção com cavidades é capaz de medir o estado do sistema das bandas laterais se o mesmo for gaussiano. Mostro também como a detecção homodina falha em medir todas as correlações entre as bandas lateiras mesmo no caso gaussiano. No capítulo 4, descrevo o aparato experimental, discutindo também como o sistema é controlado e como os dados são adquiridos. No capítulo 5, discuto em mais detalhes o emaranhamento, como ele pode ser entendido no contexto de variáveis contínuas e como podemos testar experimentalmente se nossos estados são emaranhados. No capítulo 6, discuto a teoria quântica do OPO e como podemos prever a existência de emaranhamento no nosso sistema. O capítulo 7 fecha a tese e nele 
todos os conceitos introduzidos nos capítulos anteriores são reunidos para explicar e discutir os resultados experimentais obtidos durante o doutorado. Nele discuto, além dos estados emaranhados produzidos pelo OPO e da robustez do mesmo frente a perdas, um teste de hipóteses que permite verificar a gaussianidade do nosso estado. 


\section{Resumo dos Principais Conceitos}

\subsection{Emaranhamento e Desigualdades de Bell}

O emaranhamento surgiu como fruto de uma discussão entre Einstein, Bohr e Schrödinger nos anos de 1935 e 1936. Einstein, junto com Podolsky e Rosen, propôs uma crítica à Mecânica Quântica, que ficou conhecida como paradoxo EPR [29], e que foi rapidamente respondida por Bohr [30]. Esta discussão foi expandida e desenvolvida por Schrödinger [31, 32] que foi o primeiro a utilizar o nome emaranhamento (entanglement no original em inglês). Por se tratar de correlações, somente faz sentido falar em emaranhamento para sistemas compostos por pelo menos duas partes, como dois spins ou dois osciladores harmônicos. Para estados puros, os estados emaranhados são os que não podem ser escritos como um produto tensorial das partes,

$$
\left|\psi_{A B}\right\rangle \neq\left|\psi_{A}\right\rangle \otimes\left|\psi_{B}\right\rangle
$$

Os exemplos mais simples de estados emaranhados são os estados de Bell, propostos por David Bohm [33] e definidos quando ambas as partes são sistemas de dois níveis,

$$
\left|\Psi_{ \pm}\right\rangle=\frac{1}{\sqrt{2}}\left[\left|0_{A}\right\rangle \otimes\left|1_{B}\right\rangle \pm\left|1_{A}\right\rangle \otimes\left|0_{B}\right\rangle\right], \quad\left|\Phi_{ \pm}\right\rangle=\frac{1}{\sqrt{2}}\left[\left|0_{A}\right\rangle \otimes\left|0_{B}\right\rangle \pm\left|1_{A}\right\rangle \otimes\left|1_{B}\right\rangle\right],
$$

onde 0 e 1 indexam os autovalores de um observável cujo espectro seja binário, como por exemplo $\sigma_{z}$. Para mostrar que estes estados são emaranhados, basta ver que se

$\left|\Phi_{+}\right\rangle=\left|\psi_{A}\right\rangle \otimes\left|\psi_{B}\right\rangle=\left[a\left|0_{A}\right\rangle+b\left|1_{A}\right\rangle\right] \otimes\left[c\left|0_{B}\right\rangle+d\left|1_{B}\right\rangle\right], \quad$ então $\quad a b=c d=\frac{1}{\sqrt{2}} \quad$ e $\quad a d=b c=0$, o que é impossível. Portanto, concluímos que a hipótese inicial não é válida.

Durante as décadas de 1950 e 1960, o emaranhamento foi discutido extensivamente por diversas pessoas, em especial por David Bohm, que propôs uma versão para o paradoxo EPR baseado em sistemas de spin 1/2, e John Bell [34]. Neste período, foram introduzidas teorias alternativas à Mecânica Quântica baseadas em variáveis 
ocultas ${ }^{1}[35$, 36]. Em 1963, John Bell derivou desigualdades que são satisfeitas por todas teorias de variáveis ocultas locais e podem ser testadas experimentalmente, que ficaram conhecidas como desigualdades de Bell [37]. Uma desigualdade semelhante, chamada de desigualdade $\mathrm{CHSH}^{2}$, foi proposta em 1969.

Discutirei aqui uma versão simplificada da desigualdade $C H S H$ e como ela se relaciona com o emaranhamento. Para isto considere que duas pessoas, Alice $(A)$ e Bob $(B)$, possam realizar, cada uma, duas medidas diferentes em seus respectivos sistemas físicos - $A_{1}, A_{2}, B_{1}$ e $B_{2}$ - sendo que cada medida pode resultar em somente dois valores possíveis, $a= \pm 1$ e $b= \pm 1^{3}$. Eles podem escolher fazer livremente as medidas, desde que cada um faça apenas uma por vez. Considere também que cada um receba uma das partes de um sistema físico que podem ter interagido previamente. Cada resultado das medidas $A_{i}$ e $B_{i}$ será determinado pelas próprias medidas e por um conjunto de variáveis $\lambda=\left(\lambda_{1}, \ldots, \lambda_{n}\right)^{T}$, chamadas de variáveis ocultas por serem desconhecidas por Alice e Bob ${ }^{4}$

$$
a=a\left(A_{i}, B_{j}, \lambda\right) \text { e } b=b\left(A_{i}, B_{j}, \lambda\right)
$$

Neste ponto, já há a suposição implícita de que todas as propriedades do sistema conjunto são determinadas pelo vetor $\lambda$, pois estas não podem ser afetadas pelas medidas $A_{i}$ e $B_{j}$ feitas a posteriori [5. Como Alice e Bob também desconhecem a dinâmica $\operatorname{dos} \lambda$, e como esta afeta os resultados de cada medida, supomos que esta dinâmica, seja ela qual for, é descrita por uma distribuição de probabilidades $\rho(\lambda)$, tal que as probabilidades de se observar determinados $a$ e $b$ são médias sobre as realizações de $\lambda$,

$$
a\left(A_{i}, B_{j}\right)=\int_{\Lambda} a\left(A_{i}, B_{j}, \lambda\right) \rho(\lambda) d \lambda \quad \text { e } \quad b\left(A_{i}, B_{j}\right)=\int_{\Lambda} b\left(A_{i}, B_{j}, \lambda\right) \rho(\lambda) d \lambda
$$

onde $\Lambda$ representa o conjunto de todos os $\lambda$ possíveis. Note que a medida realizada por Bob poderia afetar o resultado de Alice e vice-versa. Isto ocorre quando as medidas realizadas por um deles podem afetar o sistema do outro. Se introduzimos a hipótese adicional de que Alice e Bob estão separados de tal maneira que não seja possível qualquer forma de interação entre eles ${ }^{6}$ as medidas feitas por Alice ou Bob devem ser

\footnotetext{
${ }^{1}$ Elas dão uma interpretação clássica para a função de onda, mas para isso é necessário a introdução de um "potencial quântico" não-local para um sistema composto por duas partículas.

${ }^{2}$ Acrônimo dos nomes dos autores: Clauser, Horne, Shimony e Holt.

${ }^{3}$ Por exemplo, em um sistema de spin $1 / 2$ quando medimos o observável $2 \sigma_{z} / \hbar$.

${ }^{4}$ Cada variável $\lambda_{i}$ pode ser, em princípio, qualquer número real.

${ }^{5}$ Como também será discutido mais adiante, esta é a hipótese do Realismo.

${ }^{6}$ Como será discutido mais tarde, esta é a hipótese da Localidade.
} 
independentes para qualquer valor de $\lambda$

$$
a\left(A_{i}, B_{j}, \lambda\right)=a\left(A_{i}, \lambda\right) \quad \text { e } \quad b\left(A_{i}, B_{j}, \lambda\right)=b\left(B_{j}, \lambda\right)
$$

Da mesma forma que em (2.4), as correlações entre os resultados $a$ e $b$ medidos por Alice e Bob são $\left\langle a\left(A_{i}\right) b\left(B_{i}\right)\right\rangle=\int_{\Lambda} a\left(A_{i}, \lambda\right) b\left(B_{j}, \lambda\right) \rho(\lambda) d \lambda$. Com isso chegamos em

$$
S=\left|\left\langle a\left(A_{1}\right) b\left(B_{1}\right)\right\rangle+\left\langle a\left(A_{2}\right) b\left(B_{1}\right)\right\rangle+\left\langle a\left(A_{1}\right) b\left(B_{2}\right)\right\rangle-\left\langle a\left(A_{2}\right) b\left(B_{2}\right)\right\rangle\right| \leq 2,
$$

que é a desigualdade de CHSH. Para provar esta última passagem, basta notar que uma dentre as quantidades $a\left(A_{1}, \lambda\right) \pm a\left(A_{2}, \lambda\right)$ é necessariamente nula, pois $a\left(A_{i}, \lambda\right)= \pm 1$. Portanto, $S=\left| \pm 2\left\langle b\left(B_{i}\right)\right\rangle\right| \leq 2$. A saturação da desigualdade ocorre quando correlações perfeitas podem ser observadas entre os resultados das realizadas por Alice e Bob. Um exemplo disto ocorre quando $a\left(A_{1}\right)= \pm 1$ sempre que $b\left(B_{2}\right)= \pm 1, a\left(A_{2}\right)= \pm 1$ sempre que $b\left(B_{1}\right)= \pm 1$ e $a\left(A_{1}\right)$ e $a\left(A_{2}\right)$ estão descorrelacionadas de $b\left(B_{1}\right)$ e $b\left(B_{2}\right)$, respectivamente.

Este tipo de desigualdade foi testada experimentalmente nas décadas de 1970 e 1980 nos grupos liderados por Clauser e Horne [38, 39] e Alain Aspect [40, 41]. Nestes experimentos, não somente foram observadas violação da desigualdade de $\mathrm{CHSH}$, mas estas violações concordaram com as previsões da Mecânica Quântica. Os estados quânticos utilizados nestes experimentos são os estados de Bell. Se escolhemos o estado $\left|\Phi_{-}\right\rangle$e as medidas como

$$
A_{1}=-\frac{1}{\sqrt{2}}\left[\sigma_{z}^{A}+\sigma_{x}^{A}\right], \quad A_{2}=\frac{1}{\sqrt{2}}\left[\sigma_{z}^{A}-\sigma_{x}^{A}\right], \quad B_{2}=\sigma_{z}^{B}, \quad B_{1}=\sigma_{x}^{B} \quad \text { e } \quad B_{2}=\sigma_{z}^{B},
$$

onde $\sigma_{x}$ e $\sigma_{z}$ são as matrizes de Pauli, conseguimos a maior violação possível da desigualdade $C H S H, S=2 \sqrt{2}$. Naturalmente, por algum tempo houve uma associação dos estados emaranhados com os que violavam as desigualdades de Bell. Entretanto, o emaranhamento é uma propriedade do estado quântico e as desigualdades de Bell são vínculos que as teorias de variáveis ocultas realistas e locais devem satisfazer. As perguntas "este estado é emaranhado?" e "este estado viola desigualdades de Bell?" são diferentes. Entretanto, podemos pensar se há alguma relação entre as duas classes de estados. No ano de 1989, R. F. Werner mostra que todo sistema quântico que viola desigualdades de Bell está emaranhado, mas o inverso não é verdadeiro: há estados emaranhados que não violam desigualdades de Bell. Ele dá um exemplo de estado deste tipo que ficou conhecido como estado de Werner [42]. É também neste mesmo trabalho que ele propõe a noção atual que temos de emaranhamento para estados mistos, baseada somente em propriedades dos estados, e que será explicada em detalhes no capítulo 5 . 
É importante notar que os estados de Bell possuem correlações aparentemente perfeitas, pois, apesar de ambos Alice e Bob terem probabilidade 1/2 de observar 0 ou 1 nos seus respectivos sistemas físicos, a medida realizada por Alice leva ao conhecimento completo do estado de Bob e vice versa. Entretanto o estado global é puro, o que significa que, de acordo com os postulados da mecânica quântica, o sistema conjunto é completamente conhecido. Em outras palavras, há mais incerteza ${ }^{7}$ no estado das partes do que no estado global. Isto é algo que não ocorre em sistemas clássicos, pois a incerteza presente no todo tem um limite inferior dado pela incerteza em uma das partes, o que ocorre quando há correlações perfeitas entre as mesmas 8 [1, 44]. Neste sentido, os estados emaranhados puros possuem correlações mais fortes do que as presentes em sistemas clássicos e este é um dos motivos que eles são essenciais para protocolos como Teletransporte [2] e Codificação Superdensa [4].

\subsection{Paradoxo EPR}

Introduzido por Einstein, Podolsky e Rosen em 1935, o paradoxo EPR consiste na inconsistência lógica entre três propriedades que podem fazer parte de uma determinada teoria física: a realidade física dos elementos que a compõem, a descrição completa dada pelo vetor de estado 9 e a localidade das interações [29]. A primeira propriedade é definida pelos autores como

Se, sem perturbar o sistema em questão, podemos predizer com certeza - com probabilidade 1 - o valor de uma quantidade física, então existe um elemento de realidade correspondente a esta quantidade fisica 10

Como os próprios autores ressaltaram, está definição não esgota de forma alguma o conceito de Realismo, porém pode ser considerada como uma condição suficiente para a noção intuitiva que temos por realidade dos elementos que compõem uma teoria física.

\footnotetext{
${ }^{7}$ Uso o termo "incerteza" aqui como sinônimo de falta de conhecimento. Estes conceitos são matematicamente traduzidos na idea de entropia de Shannon, $S_{S}=\sum_{i} p_{i} \ln p_{i}$ ou, no caso de sistemas quânticos, na entropia de Von Neumann, $S_{V N}=\operatorname{tr}[\rho \ln \rho]$, onde $\rho$ é o operador densidade [43, 44].

${ }^{8}$ Esta afirmação está relacionada com a noção de informação mútua [44]. Em sistamas clássicos, quando as partes que o compõe estão perfeitamente correlacionadas, a incerteza no sistema global é igual ao máximo entre as incertezas de cada parte. Quando as mesmas são descorrelacionadas, a incerteza no sistema global se iguala à soma das incertezas nas partes. Uma situação em que a incerteza no sistema global é menor de que em ambas as partes não faz sentido em uma teoria probabilística clássica e levaria a correlações mais fortes que correlações perfeitas.

${ }^{9}$ Por motivos históricos, os autores se referem à função de onda em vez do vetor de estado. Por isso, nas citações feitas no decorrer do texto manterei a terminologia do artigo original.

${ }^{10}$ Tradução própria.
} 
Essa noção está presente em todas as teorias clássicas como a Mecânica Newtoniana e o Eletromagnetismo. A segunda propriedade é um dos postulados da Mecânica Quântica [45, 46, 47]. Eles também propõe uma condição que deve ser satisfeita por uma teoria física completa,

\section{Todo elemento de realidade física deve ter uma contrapartida na teoria física ${ }^{11}$.}

e que na Mecânica Quântica está relacionada aos vetores de estado que representam auto-estados de operadores hermitianos.

A terceira, que pode ser traduzida como sistemas que não interagem não podem se afetar mutuamente, é uma condição extremamente razoável e satisfeita por qualquer teoria física existente. No exemplo proposto por EPR, é assumido que duas partículas interagem por um período de tempo e depois disso deixam de interagir. Entretanto, em um experimento real, surge o problema de como garantir que não há interação ou que, se houver tais interações, elas são desprezíveis. Este tipo de questionamento motivou diversas críticas aos primeiros trabalhos que buscavam medir violações das desigualdades de Bell e ficou conhecido como Locality Loophole [41]. Para garantir que não haja interações, foi proposto que as partículas fossem separadas de modo que cada uma estivesse fora do horizonte de eventos da outra durante a realização do experimento. Entretanto, quando esta condição não é satisfeita em experimentos reais, não é possível apoiar-se na Relatividade Restrita para garantir a localidade. Medidas de violação de desigualdades de Bell que satisfazem esta condição somente foram obtidas recentemente [48]. Desta maneira a localidade é garantida independentemente da interação específica e baseada somente na constância da velocidade da luz ${ }^{12}$, que é um fato experimental muito bem verificado e cujo valor já é conhecido com bastante acurácia há mais de cem anos [49 13 . Mais detalhes sobre o conceito de localidade, em especial sobre suas sutilizas, e outros conceitos correlatos, como a não-sinalização, podem ser encontrados em [50, 51, 52, 53]

Para demonstrar o paradoxo, os autores provam dois resultados. O primeiro é que apenas uma das assertivas a seguir pode ser verdadeira: 1 - A descrição da realidade física dada pela função de onda não é completa e 2 - Duas quantidades físicas descritas por operadores que não comutam não possuem realidade física simultânea. As ideias que levam a esta conclusão são bem simples e podem ser entendidas como consequências diretas

\footnotetext{
${ }^{11}$ Tradução própria.

${ }^{12} \mathrm{~A}$ existência de um horizonte de eventos é consequência direta deste fato.

${ }^{13}$ Atualmente, ela é considerada exata e é usada para definir o metro.
} 
das relações de incerteza e da noção de realidade física proposta por EPR. O segundo resultado mostra que é possível, assumindo que a descrição dada pelo vetor de estado seja completa (que a assertiva 1 seja falsa), associar dois vetores de estado diferentes à mesma realidade física. Como estes dois vetores de estado podem ser auto estados de operadores que não comutam, isto equivale a dizer que as quantidades descritas por estes operadores possuem realidade física simultânea. Consequentemente, a negação da assertiva 1 leva à negação da 2 e, portanto, a conclusão final é de que 1 deve ser verdadeira: a descrição dada pela função de onda não é completa. Com este último argumento, é demonstrado o paradoxo. A noção de realidade física foi utilizada de forma explícita para provar o primeiro resultado e a de localidade será usada para provar o segundo.
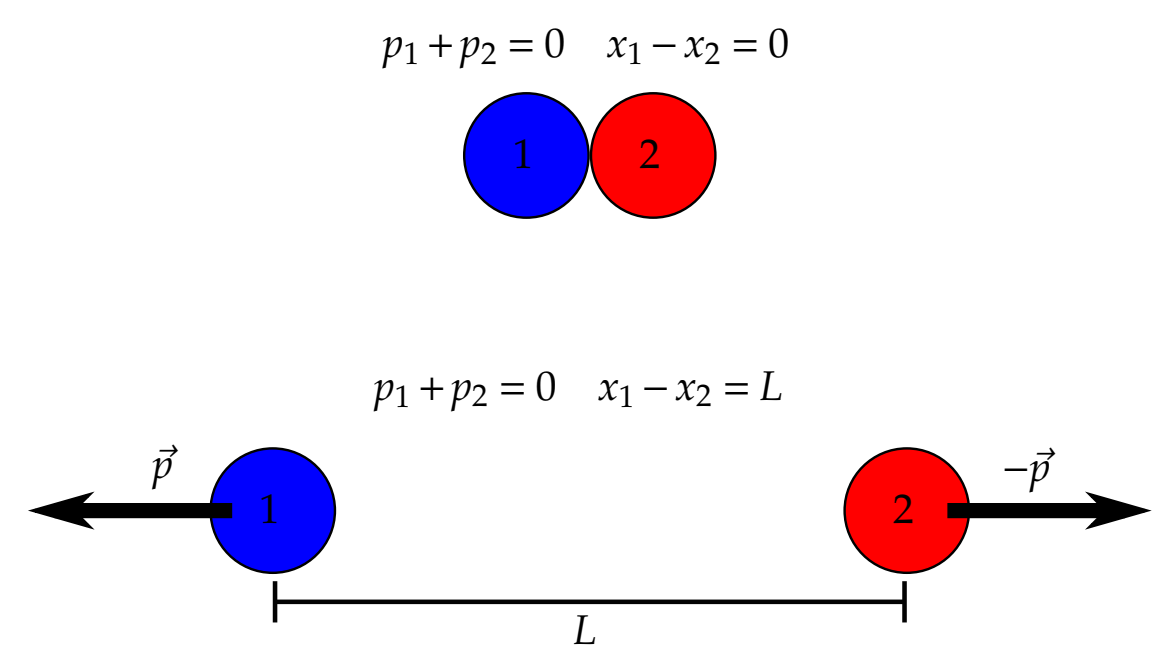

Figura 2.1: Exemplo interação física que produz um estado EPR. As partículas 1 e 2 podem ser pensadas como partes de um mesmo sistema global, inicialmente em repouso. Após uma explosão, ambas são enviadas em sentidos opostos com o mesmo valor absoluto de momento linear.

Para derivar este segundo resultado, os autores utilizam como exemplo um estado emaranhado de duas partículas, supondo que não haja interação entre elas. Considere duas partículas de mesma massa em uma dimensão, como mostrado na figura 2.1, se afastam ambas com a mesma velocidade. Como a soma dos momentos e a subtração das posições são bem conhecidas, e as mesmas grandezas comutam, a função de onda do sistema conjunto será

$$
\psi\left(x_{-}, p_{+}\right)=\delta\left(x_{-}-L\right) \delta\left(p_{+}\right), \quad \text { onde } \quad x_{-}=x_{1}-x_{2} \quad \text { e } \quad p_{+}=p_{1}+p_{2}
$$

O que significa que as partículas possuem momentos iguais e de sinais opostos em todos os instantes de tempo e, como consequência, a diferença entre as posições aumenta a 
uma taxa constante. Como a soma dos momentos e a subtração das posições é determinada, apenas o conhecimento da posição (momento) de uma partícula é suficiente para saber a posição (momento) da outra. Desta forma, podemos escolher medir a posição da partícula 1 e o momento da partícula 2. Como estes estão correlacionados como mostrado em (2.8), podemos inferir os momentos e posições de ambas as partículas com precisão arbitrária, ou seja, com probabilidade 1, o que equivale a dizer que as posições e momentos de ambas as partículas possuem realidade física simultânea. Com este exemplo, provamos o segundo resultado mencionado no parágrafo anterior. Esta correlação - aparentemente perfeita como no caso dos Estados de Bell - também é um exemplo de emaranhamento. No estado (2.8), a soma dos momentos e a subtração das posições é conhecida com precisão absoluta, o que faz com que este não seja fisicamente realizável. Entretanto, ele é o caso limite do estado

$$
|\psi\rangle=\frac{1}{\cosh (\lambda)} \sum_{n=0}^{\infty} \tanh ^{n}(\lambda)|n, n\rangle,
$$

quando $\lambda \rightarrow \infty$, onde $|n\rangle$ são os autoestados do operador número que formam a base de Fock e $\theta$ é um número real. Em Ótica Quântica, este tipo de estado é conhecido como estado comprimido de dois modos e é produzido por diversos sistemas físicos como o oscilador paramétrico ótico (OPO), que é um dos temas centrais deste trabalho [14, 54].

A resposta dada por Bohr critica a noção de realismo utilizada por EPR. Ele ressalta que os pontos levantados por EPR não apontam para inconsistências na estrutura interna da Mecânica Quântica, e sim quando esta é considerada em conjunto com as noções de realismo e localidade. Segundo a Mecânica Quântica, os observáveis propostos por EPR para provar o paradoxo, podem ser medidos ao mesmo tempo com precisão arbitrariamente alta, pois, apesar de $x_{1}$ e $p_{1}$ ou $x_{2}$ e $p_{2}$ não comutarem, os observáveis $x_{+}$e $p_{-}$, mostradas em (2.8), comutam.

\subsection{Oscilador Paramétrico Ótico e Conversão Paramétrica Descendente}

O OPO é o sistema experimental com que trabalhamos e, como dito antes, pode produzir o estado comprimido de dois modos. O OPO consiste em um cristal nãolinear colocado dentro de uma cavidade ótica e bombeado por um laser. Devido à não-linearidade de segunda ordem, o cristal absorve fótons do laser de bombeio com uma pequena probabilidade. Para cada fóton do bombeio absorvido, o cristal emite 
dois fótons cuja soma das energias é igual à energia do fóton original ${ }^{14}$,

$$
\hbar \omega_{0}=\hbar \omega_{2}+\hbar \omega_{1}
$$

Por serem emitidos ao mesmo tempo, estes fótons são chamados de fótons gêmeos e o processo físico que os produz é conhecido como conversão paramétrica descendente (PDC- do inglês parametric down converrion).

A cavidade ótica é formada por dois espelhos, alinhados de modo a fazer com que a luz seja refletida sobre ela mesma, e atua como um amplificador. Ao fazer os fótons produzidos pela PDC voltarem ao cristal, a cavidade aumenta a probabilidade de absorção de um fóton do bombeio devido à presença de mais fótons gêmeos ${ }^{15}$. Da mesma forma que ocorre em um laser, quando o ganho introduzido pelo cristal supera as perdas intracavidade o sistema oscila e o OPO emite luz nas frequências dos fótons gêmeos.

Meios lineares não são capazes de produzir conversão de frequências, pois a polarização induzida é proporcional ao campo elétrico. Quando o meio é não-linear, a polarização é função de potências do campo. Isto faz com que, quando um feixe de luz de frequência $\omega_{0}$, incide em um meio não-linear, a polarização induzida no meio seja [55, 56]

$$
P_{i}=\chi_{i j}^{(1)} A_{j}+\chi_{i j k}^{(2)} A_{j} A_{k}+\chi_{i j k l}^{(3)} A_{j} A_{k} A_{l}+\ldots
$$

onde $\chi^{(n)}$ são os tensores de susceptibilidade elétrica de ordem $n$ e os índices repetidos estão sendo somados e $A_{j}$ representa uma componente do campo elétrico. Um meio não-linear de ordem 2 possui $\chi^{(2)}$ grande o suficiente para que os primeiros efeitos de conversão de frequência sejam observados. O mais simples deles é a geração de segundo harmônico, que consiste na emissão de luz com o dobro da frequência da luz incidente. Como pode ser visto em (2.11), um campo incidente descrito por $A_{\text {in }}(t)=\operatorname{Re}\left[\alpha_{i n} \mathrm{e}^{-i \omega_{0} t}\right]$, vai induzir uma polarização de

$$
P_{\text {ind }}(t)=\chi^{(1)} \operatorname{Re}\left[\alpha_{i n} \mathrm{e}^{-i \omega_{0} t}\right]+\chi^{(2)} \operatorname{Re}\left[\alpha_{\text {in }}^{2} \mathrm{e}^{-2 i \omega_{0} t}\right]+\chi^{(2)}\left|\alpha_{\text {in }}\right|^{2}
$$

em um meio não-linear de segunda ordem em que $\chi_{i j k}^{(2)}=\chi^{(2)} \delta_{i j} \delta_{i k}$. Além do segundo harmônico, a não-linearidade induz uma polarização estática no meio, conhecida como retificação ótica.

\footnotetext{
${ }^{14} \mathrm{Na}$ verdade, há uma eficiência associada a este processo e que não é $100 \%$. Há fótons absorvido do bombeio que não são convertido para fótons gêmeos.

${ }^{15}$ Este efeito é um tipo de emissão estimulada: a probabilidade de emissão aumenta com o número de fótons.
} 
Outros efeitos ocorrem quando a luz incidente possui duas componentes de frequência,

$$
A_{\text {in }}(t)=\operatorname{Re}\left[\alpha_{1} \mathrm{e}^{-i \omega_{1} t}\right]+\operatorname{Re}\left[\alpha_{2} \mathrm{e}^{-i \omega_{2} t}\right] .
$$

Neste caso, a polarização induzida será

$$
\begin{aligned}
P_{\text {ind }}(t) & =\chi^{(1)}\left\{\operatorname{Re}\left[\alpha_{1} \mathrm{e}^{-i \omega_{1} t}\right]+\operatorname{Re}\left[\alpha_{2} \mathrm{e}^{-i \omega_{2} t}\right]\right\}+\chi^{(2)}\left\{\operatorname{Re}\left[\alpha_{1}^{2} \mathrm{e}^{-2 i \omega_{1} t}\right]+\operatorname{Re}\left[\alpha_{2}^{2} \mathrm{e}^{-2 i \omega_{2} t}\right]\right\} \\
& +2 \chi^{(2)}\left\{\operatorname{Re}\left[\alpha_{1} \alpha_{2} \mathrm{e}^{-i\left(\omega_{1}+\omega_{2}\right) t}\right]+\operatorname{Re}\left[\alpha_{1} \alpha_{2}^{*} \mathrm{e}^{-i\left(\omega_{1}-\omega_{2}\right) t}\right]+\left|\alpha_{1}\right|^{2}+\left|\alpha_{2}\right|^{2}\right\}
\end{aligned}
$$

Além do segundo harmônico e retificação ótica, há a geração de soma e diferença de frequências. Se consideramos que $\omega_{1}+\omega_{2}=\omega_{0}$, vemos que o processo de geração de soma de frequências não é reversível segundo a descrição da ótica não-linear clássica, pois um meio de segunda ordem, quando excitado por luz oscilando na frequência $\omega_{0}$, irá produzir apenas o seu segundo harmônico. Por outro lado, se também houver luz na frequência $\omega_{1}$, o meio produz campos oscilando com $\omega_{2}$ devido à geração de diferença de frequências. Em outras palavras, os processos de geração de sub-harmônicos são estimulados na ótica não-linear clássica, pois é necessária a presença de outro subharmônico, agindo como uma semente, para que eles ocorram.

Quando pensamos na geração de soma de frequências em termos de fótons, o meio não-linear absorve dois fótons com energias $\hbar \omega_{1}$ e $\hbar \omega_{2}$ e emite um fóton, cuja energia é a soma das duas, como em 2.10). Como há conservação de energia, o processo deve ser reversível mesmo na ausência de qualquer sub-harmônico, diferentemente do que prevê a ótica não-linear clássica. Este processo inverso é a PDC [57] e, assim como a emissão espontânea, ela não pode ser entendida somente por meio do eletromagnetismo clássico. Na PDC, o papel da semente é feito pelo vácuo e por isso ela é chamada de um processo espontâneo. Neste sentido, a PDC possui a mesma similaridade com a geração de diferença de frequências que a emissão espontânea tem com a emissão estimulada.

Além da conservação de energia, também há conservação de momento na $P D C$, conhecida também como acordo de fase,

$$
\mathbf{k}_{0}=\mathbf{k}_{1}+\mathbf{k}_{2}
$$

onde $\mathbf{k}_{0}$, $\mathbf{k}_{1}$ e $\mathbf{k}_{2}$ são os momentos do bombeio e dos fótons gêmeos. Eles podem ser emitidos com polarizações iguais ou ortogonais, dependendo das características do cristal 16 . O acordo de fase é importante quando há propagação e é mais crítico quanto

\footnotetext{
${ }^{16}$ Os acordos de fase mais comuns são os do tipo I, quando os fótons são gerados com a mesma
} 

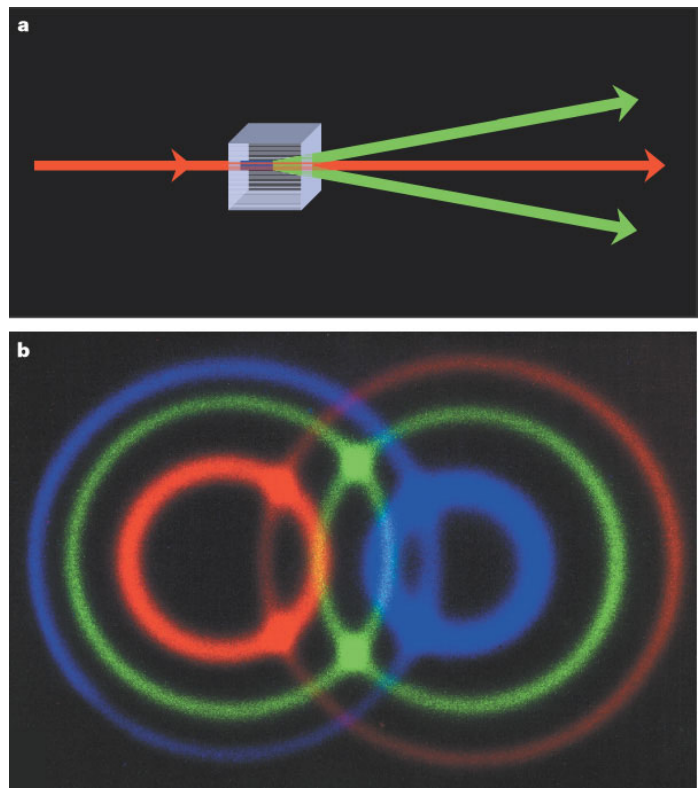

Figura 2.2: O bombeio excita o cristal e gera cones de fluorescência que satisfazem as relações de conservação de energia e acordo de fase. As cores dos cones representam diferentes acordos de fase. Figura retirada de [58].

maior for o tamanho do cristal.

\subsection{Cavidades Óticas}

Quando um cristal é colocado dentro da cavidade ótica, os fótons emitidos pela conversão paramétrica dão origem a um feixe de luz intenso quando a potência de bombeio está acima de um determinado limiar de oscilação, da mesma forma como ocorre em um laser. Como utilizamos cavidades óticas Fabry-Perot também para outros propósitos no experimento além do $O P O$, discutirei agora a cavidade vazia e mais adiante vou juntá-la com a PDC para descrever o OPO.

Uma cavidade Fabry-Perot é formada por espelhos posicionados de modo a fazer com que a luz incidente retorne sobre si mesma após ser refletida por todos os espelhos da cavidade. Para que haja interferência, a luz refletida após dar uma volta pela cavidade deve ser superpor à luz incidente em todos os graus de liberdade [56, 59]. Cavidades Fabry-Perot podem ser de dois tipos: linear e em anel. A primeira é caracterizada pelo fato do campo se propagar em dois sentidos antes de sair da cavidade, resultando necessariamente na formação de ondas estacionárias. Na segunda, o campo pode se propagar em apenas um sentido e necessita de, no mínimo, três espelhos para polarização, ou do tipo II, quando as polarizações de ambos são diferentes. 
ser construída. Por uma questão didática, utilizarei ondas planas para explicar o comportamento da luz nestas cavidades. No experimento, os feixes de luz que utilizamos não são adequadamente descritos por ondas planas, mas por feixes gaussianos [56]. Entretanto, a descrição mais simples usando ondas planas captura todas as propriedades físicas importantes para o trabalho discutido no texto.
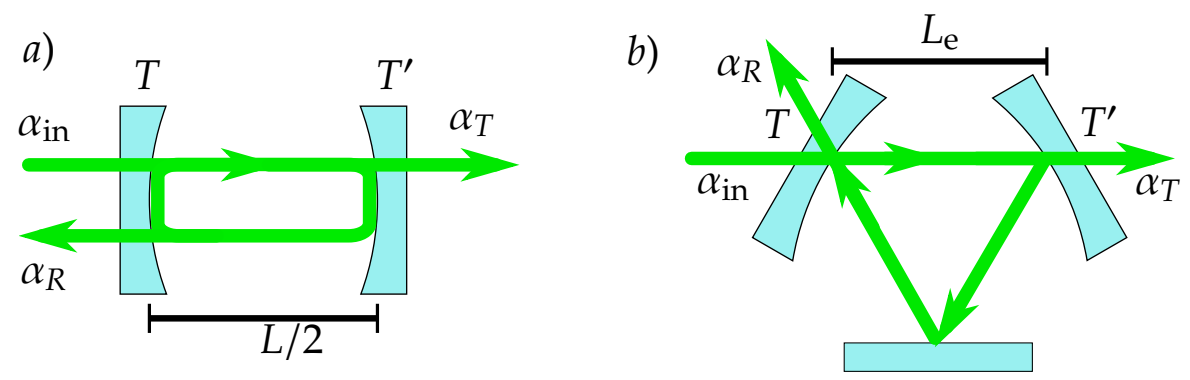

Figura 2.3: a) cavidade Fabry-Perot linear simples: a diferença nas trajetórias dos campos que se propagam em ambos os sentidos dentro da cavidade é meramente ilustrativa. Em uma cavidade real, estes campos estão superpostos. b) cavidade Fabry-Perot em anel: o campo que se propaga em somente um sentido dentro da cavidade.

Considere uma cavidade formada por dois espelhos com coeficientes de transmissão $T$ e $T^{\prime}$, coeficientes de reflexão $R=1-T$ e $R^{\prime}=1-T^{\prime}$ e de comprimento total $L$. Ela pode representar tanto uma cavidade linear simples com os dois espelhos posicionados à distância $L / 2$ ou uma cavidade em anel com dois espelhos parcialmente refletores como mostrado na figura 2.3. Ao incidir um campo de frequência $\omega=k c$ na cavidade, uma fração do mesmo é refletida diretamente pelo primeiro espelho enquanto o resto é transmitido, entrando na cavidade. Após uma volta completa pela cavidade, parte deste campo é transmitido pelo primeiro espelho e se superpõe ao campo refletido diretamente. A outra parte é refletida e se propaga em uma segunda volta. Este processo se repete sucessivamente fazendo com que o campo dentro da cavidade logo após ser refletido pelo primeiro espelho seja igual a 17

$$
\alpha_{n}=\sqrt{T} \alpha_{\text {in }}-\sqrt{R}\left(-\sqrt{R^{\prime}} \mathrm{e}^{i k L} \alpha_{n-1}\right)
$$

após a n-ésima volta pela cavidade. Cada um dos espelhos funciona como um divisor de feixes e a expressão anterior é uma das equações que caracterizam a transformação de divisor de feixes [62], como mostrada na figura 2.4

$$
d=\sqrt{R} a+\sqrt{T} b, \quad c=\sqrt{T} a-\sqrt{R} b,
$$

\footnotetext{
${ }^{17} \mathrm{O}$ sinal negativo é devido à fase de $\pi$ que o campo elétrico recebe ao ser refletido por um meio com índice de refração maior [60,61].
} 


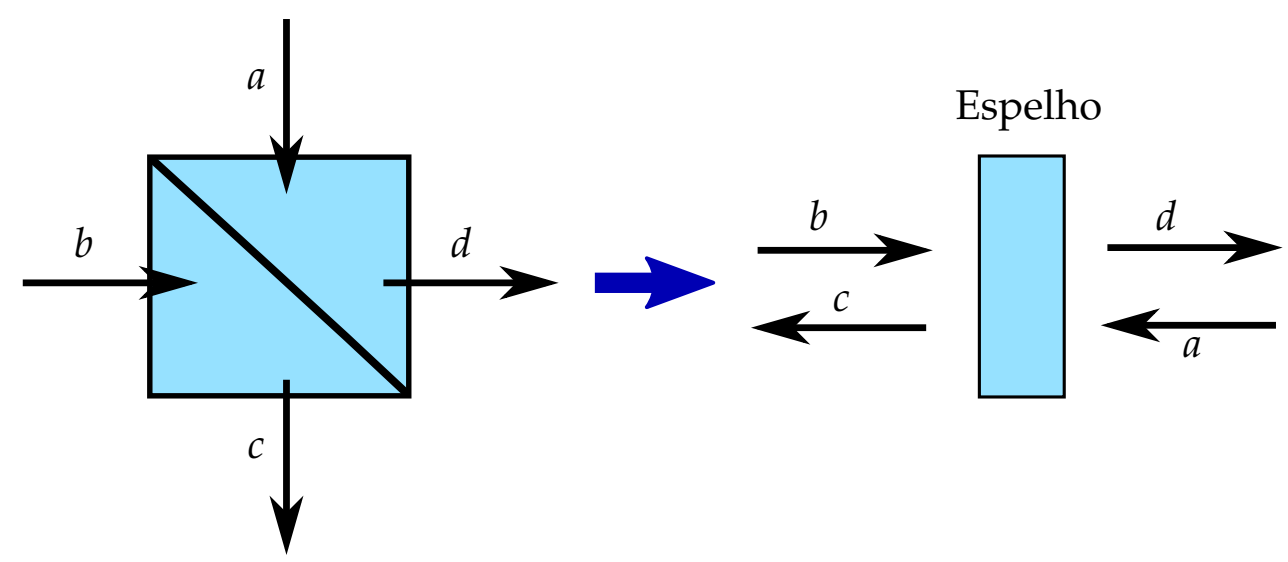

Figura 2.4: Transformação de divisor de feixes: Os campos de saída c e d são resultado da interferência entre os campos de entrada a e $b$.

onde as quantidades $a, b, c$ e $d$ são as amplitudes do campo elétrico ${ }^{18}$, A primeira equação em (2.17) aplicada ao primeiro espelho se torna

$$
\alpha_{R}=\sqrt{R} \alpha_{\text {in }}+\sqrt{T}\left(-\sqrt{R^{\prime}} \mathrm{e}^{i k L} \alpha_{n-1}\right)
$$

No limite em que $n \rightarrow \infty$, o campo intracavidade chega em uma situação estacionária, tal que $\alpha_{n}=\alpha_{n-1}$. Portanto, a partir de (2.16) e (2.18), os campos intracavidade logo após o primeiro espelho e o campo refletido se relacionam com o campo incidente como

$$
\alpha=\frac{\sqrt{T}}{1-\sqrt{R R^{\prime}} \mathrm{e}^{i k L}} \alpha_{\text {in }} \quad \text { e } \quad \alpha_{R}=r(k L) \alpha_{\text {in }} \quad \text { onde } \quad r(k L)=\frac{\sqrt{R}-\sqrt{R^{\prime}} \mathrm{e}^{i k L}}{1-\sqrt{R R^{\prime}} \mathrm{e}^{i k L}} .
$$

O campo refletido também pode ser entendido como o resultado da interferência do campo refletido diretamente pelo primeiro espelho com os campos transmitidos pelo mesmo após as diversas voltas pela cavidade,

$$
\alpha_{R}=\lim _{n \rightarrow \infty} \alpha_{R 0}+\alpha_{R 1}+\alpha_{R 2}+\ldots+\alpha_{R n}
$$

onde $\alpha_{R 0}=\sqrt{R} \alpha_{\text {in }}$ é o campo refletido diretamente e $\alpha_{R 1}=-\sqrt{T} \mathrm{e}^{i 2 k L} \sqrt{R^{\prime}} \sqrt{T} \alpha_{\text {in }}$ é o campo transmitido pelo primeiro espelho após uma volta pela cavidade. O campo transmitido após $n$ voltas, $\operatorname{com} n \geq 1$, é

$$
\alpha_{R n}=(-1)^{n} T \mathrm{e}^{2 n i k L} \sqrt{R^{n-1}} \sqrt{R^{\prime}} n \alpha_{\text {in }} .
$$

A soma infinita em 2.20 é da forma $A+B \sum_{n} x^{n}$ e converge para $A+B(1+x)^{-1}$ quando $|x|<1$. Consequentemente, chegamos na mesma expressão para o campo refletido

\footnotetext{
${ }^{18}$ em um sistema quântico, elas são os operadores de aniquilação.
} 
mostrada em (2.19), pois $R$ e $R^{\prime}<1$.
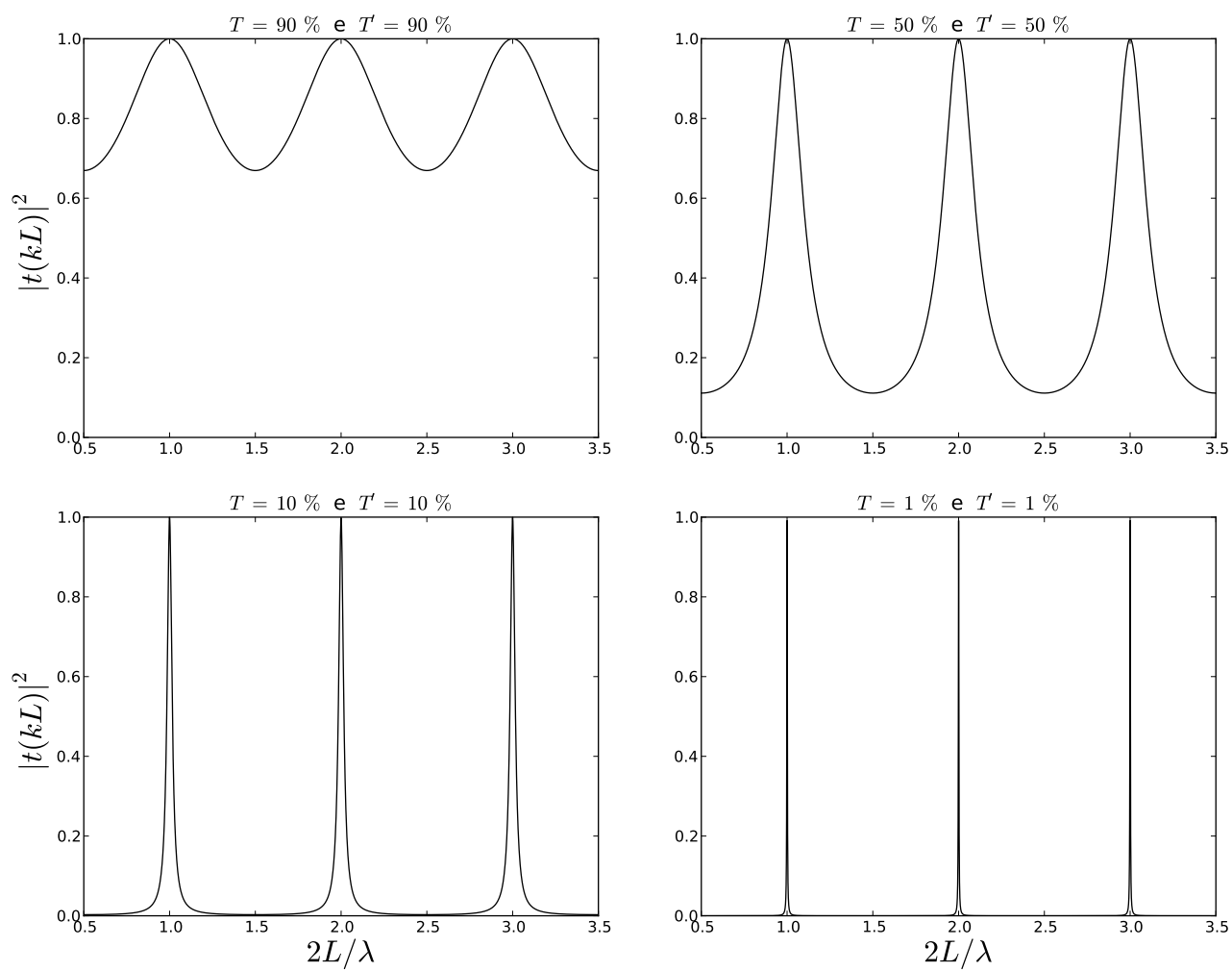

Figura 2.5: Coeficientes de transmissão de uma cavidade Fabry-Perot para diversos $T$ e $T^{\prime} . \grave{A}$ medida que a cavidade se torna mais fechada, o perfil do campo transmitido varia de uma senóide para uma sequência de Lorentzianas, de largura cada vez mais estreitas, em torno dos modos normais. Para uma cavidade completamente fechada, recuperamos somente os modos normais.

Partindo de um raciocínio similar chega-se à conclusão de que o campo transmitido é

$$
\alpha_{T}=t(k L) \alpha_{\text {in }}, \quad t(k L)=\frac{\sqrt{T T^{\prime}} \mathrm{e}^{i k L_{\mathrm{e}}}}{1-\sqrt{R R^{\prime}} \mathrm{e}^{i k L}},
$$

onde $L_{\mathrm{e}}$ é a distância entre os dois espelhos. Para uma cavidade linar simples, $L_{\mathrm{e}}=L / 2$ e pode ser ajustada para uma cavidade em anel, tal que $0<L_{\mathrm{e}}<L / 2$. O coeficiente de transmissão, entretanto, não depende deste parâmetro,

$$
|t(k L)|^{2}=\frac{T T^{\prime}}{1+R R^{\prime}-2 \sqrt{R R^{\prime}} \cos (k L)} .
$$

A cavidade Fabry-Perot pode ser entendida como um divisor de feixes dependente da frequência e os coeficientes de transmissão e reflexão satisfazem a relação de conservação de energia $|r|^{2}+|t|^{2}=1$. O parâmetro $k L=2 \pi L / \lambda$ mede a quantidade de oscilações que cabem dentro da cavidade. Os modos normais (ou ressonantes) da cavidade são caracterizados por $\lambda_{n}=L / n$ com " $n$ " natural. 
Quando $R=1$, o campo incidente é completamente refletido no primeiro espelho. Este é um caso limite em que não há cavidade de fato, pois o primeiro espelho é completamente refletor. Se $R^{\prime}=1$ e $R<1$ toda a luz também será completamente refletida, porém ela entra na cavidade antes. Neste caso, $r(k L)=\mathrm{e}^{i \phi(k L)}$, fazendo com que o campo refletido receba uma fase dependente da frequência em relação ao campo incidente. Este tipo de cavidade é chamada de cavidade sem perdas, pois a intensidade do campo refletido é igual à do incidente. Outro caso limite ocorre quando $R=R^{\prime}$ e é caracterizado por uma supressão total do campo refletido quando a cavidade está em ressonância. Este caso é conhecido como cavidade com casamento de impedâncias ${ }^{19}$ e ocorre devido à interferência destrutiva entre o campo refletido diretamente pelo primeiro espelho e os refletidos após entrarem na cavidade.

A diferença de frequência entre modos ressonantes adjacentes é

$$
\Delta f=\frac{c}{\lambda_{n+1}}-\frac{c}{\lambda_{n}}=\frac{c}{L}=\frac{1}{\tau}
$$

e somente depende do tamanho da cavidade. Ela é chamada de intervalo espectral livre (FSR - do inglês free spectral range) e é igual ao inverso do tempo que a luz leva para dar uma volta pela cavidade. A largura à meia altura do pico de transmissão, chamada de largura de banda, é proporcional ao FSR da cavidade e é dada por

$$
\delta f=\frac{\Delta f}{\mathcal{F}} \quad \operatorname{com} \quad \mathcal{F}=\pi \operatorname{arcsen}\left[\frac{\sqrt{1+R R^{\prime}}}{2\left(R R^{\prime}\right)^{1 / 4}}\right]^{-1} .
$$

O inverso desta constante de proporcionalidade é chamado de finesse da cavidade. Ela mede o quão estreitos são os picos de ressonância em relação ao FSR e é função somente das transmissões dos espelhos. Note que $\mathcal{F}$ aumenta à medida que $T$ e $T^{\prime}$ diminuem $\mathrm{e}$

$$
\mathcal{F} \approx \frac{2 \pi\left(R R^{\prime}\right)^{1 / 4}}{\sqrt{1+R R^{\prime}}} \approx \frac{2 \pi}{T+T^{\prime}}
$$

quando $T$ e $T^{\prime} \rightarrow 0$, o que é chamado de regime de alta finesse.

As cavidades que usamos no labotatório medem entre dezenas de milímetros e unidades de metro. Isso faz com que nelas caibam entre $10^{4}$ e $10^{6}$ comprimentos de onda $\mathrm{a}^{20}$. Este regime está bem longe do mostrado na figura 2.6 e nele faz pouca diferença em torno de qual modo normal específico estaremos trabalhando. Podemos escrever o

\footnotetext{
${ }^{19}$ Esse nome vem do paralelo que existe com circuitos elétricos em que os espelhos são substituídos por capacitores.

${ }^{20}$ Os comprimentos de onda no domínio ótico vão de $380 \mathrm{~nm}$ para o violeta a $3 \mu \mathrm{m}$ para o infravermelho próximo. No nosso experimento, utilizamos $532 \mathrm{~nm}$ (verde) e $1064 \mathrm{~nm}$ (infravermelho).
} 
argumento de $r$ e $t$ de uma forma mais conveniente como

$$
k L=\frac{2 \pi f}{c} L=2 \pi \frac{L}{c} \delta f \frac{f}{\delta f}=\frac{2 \pi f^{\prime}}{\mathcal{F}},
$$

onde $f^{\prime}=f / \delta f$ é a frequência em unidades de largura de banda. Como o sistema é
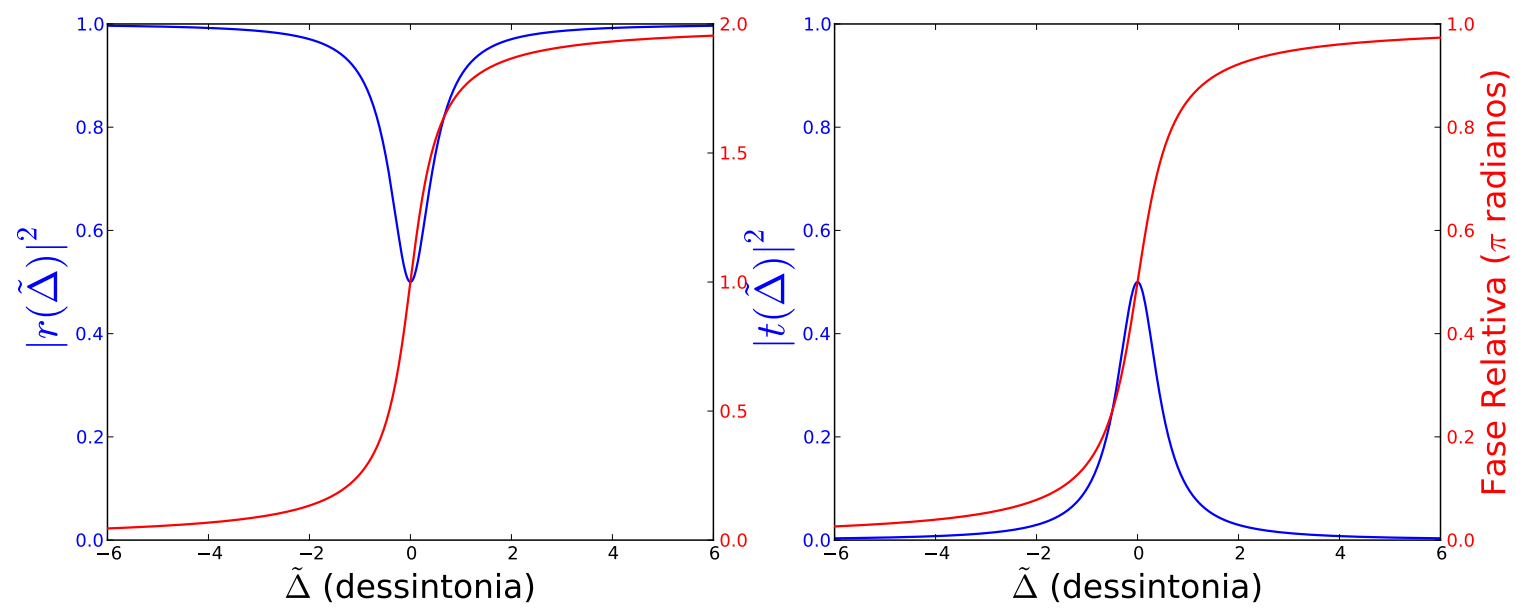

Figura 2.6: Varredura de uma cavidade linear simples em torno de uma ressonância no regime de alta finesse. Os coeficientes de reflexão e transmissão são mostrados em azul e a fase introduzida pela cavidade entre os mesmos e o campo incidente estão em vermelho. Note que a fase do campo refletido sofre uma rotação completa, de $2 \pi$, ao passar pela ressonância enquanto a fase do campo transmitido varia somente a metade. Esses gráficos foram feitos com $R_{\min }=0,5$.

periódico, qualquer deslocamento na frequência por um múltiplo inteiro do FSR não o modifica. Sendo assim, podemos substituit $f^{\prime}$ pela dessintonia em unidades de larguda de banda, $\widetilde{\Delta}=f^{\prime}-f_{0}^{\prime}$, onde $f_{0}^{\prime}$ é a frequência de ressonância mais próxima. No limite de alta Finesse e em torno de uma ressonância, $\mathcal{F}>>\widetilde{\Delta}$, fazendo com que os coeficientes $r$ e $t$ sejam simplificados para

$$
r(\tilde{\Delta})=-\frac{\sqrt{R_{\min }}+2 i \tilde{\Delta}}{1-2 i \tilde{\Delta}}, t(\tilde{\Delta})=\frac{\sqrt{T_{\max }}}{1-2 i \tilde{\Delta}} \operatorname{com} R_{\min }=\left(\frac{T-T^{\prime}}{T+T^{\prime}}\right)^{2} \text { e } T_{\max }=\frac{4 T T^{\prime}}{\left(T+T^{\prime}\right)^{2}}
$$

onde $R_{\min }$ e $T_{\max }$ são os coeficientes de reflexão e transmissão em ressonância. Devido à conservação de energia, $R_{\min }+T_{\max }=1$. Estes coeficientes estão mostrados na figura 2.6. Como dito anteriormente, a cavidade introduz uma fase entre o campo refletido e o campo incidente. Para uma cavidade com perdas, há também uma atenuação do campo refletido e um aumento do campo transmitido ao passar pela ressonância. Neste caso, o campo trasmitido também recebe uma fase relativa ao campo incidente. 


\subsection{OPO Clássico}

Como dito anteriormente, o OPO é formado pelo cristal não-linear de segunda ordem inserido dentro de uma cavidade ótica. Vou descrever aqui o OPO utilizando campos clássicos, pois neste contexto diversas propriedades do sistema podem ser entendidas de maneira mais simples. A descrição quântica será dada no capítulo 6 . Na descrição clássica do OPO considera-se que o campo intracavidade seja formado pelo bombeio e pelos feixes gêmeos, o que torna possível calcular a intensidade média de ambos os campos ao sair da cavidade. A discussão que farei aqui é baseada em [63] 21. Um cristal não-linear de segunda ordem é excitado por três feixes de luz com amplitudes e frequências iguais a $\alpha_{j}$ e $\omega_{j}$, onde $j=0,1,2$ representam bombeio, sinal e complementar respectivamente.

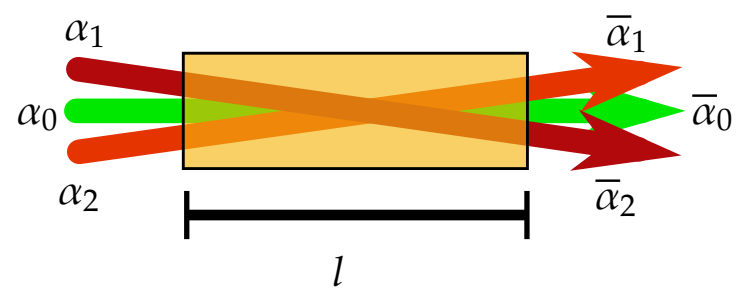

Figura 2.7: Três campos interagindo dentro do cristal. O ângulo entre eles é meramente ilustrativo.

Ao passar pelo cristal, parte da intensidade do bombeio é absorvida e emitida nas frequências de sinal e complementar, devido à interação não-linear. Esta absorção é muito pequena e introduz atenuação significativa. O campo de bombeio após o cristal está relacionado com o de entrada como

$$
\bar{\alpha}_{0}=\alpha_{0} \mathrm{e}^{i \omega_{0} l / n_{c} \mathcal{c}}+P_{\text {ind } 0}=\alpha_{0} \mathrm{e}^{i \omega_{0} l / n_{c} \mathcal{c}}-2 \chi \alpha_{1} \alpha_{2} \mathrm{e}^{i\left(\omega_{1}+\omega_{2}\right) l / n c},
$$

onde $n$ é o índice de refração do cristal e $\chi$ é uma susceptibilidade de segunda ordem efetiva e é função de $\chi^{(1)}$ e $\chi^{(2)}[64,65,63]$. Comparando com a expressão $(2.14)$, vemos que a polarização linear é responsável pelo índice de refração [61, 55]. A polarização não-linear de segunda ordem é responsável pelo segundo termo em (2.29). Apenas o termo referente à soma de frequências é mantido, pois ele é o único em fase com o bombeio em todas as posições a medida que os três campos se propagam pelo cristal. Os

\footnotetext{
${ }^{21}$ Uso aqui algumas simplificações. Por exemplo, considero o campo como uma onda plana e não como um feixe gaussiano. Apesar desta aproximação não ser estritamente válida no experimento, ela simplifica bastante as contas e retém os aspectos físicos mais importantes.
} 
outros termos não-lineares contribuem positivamente ou negativamente dependendo da posição dentro do cristal, fornecendo uma contribuição efetiva nula ${ }^{22}$, O sinal negativo entre o primeiro e segundo termos de (2.29) é colocado por conveniência. $\mathrm{O}$ importante é que haja uma diferença entre este sinal e os que aparecem nas equações 2.3023 Para sinal e complementar,

$$
\bar{\alpha}_{1}=\alpha_{1} \mathrm{e}^{i \omega_{1} n_{c} l / c}+2 \chi \alpha_{0} \alpha_{2}^{*} \mathrm{e}^{i\left(\omega_{0}-\omega_{2}\right) l / n_{c} c} \quad \text { e } \quad \bar{\alpha}_{2}=\alpha_{2} \mathrm{e}^{i \omega_{2} n_{c} l / c}+2 \chi \alpha_{0} \alpha_{1}^{*} \mathrm{e}^{i\left(\omega_{0}-\omega_{1}\right) l n_{c} c}
$$

Note que agora o termo que mantém o acordo de fase corresponde à diferença de frequências.

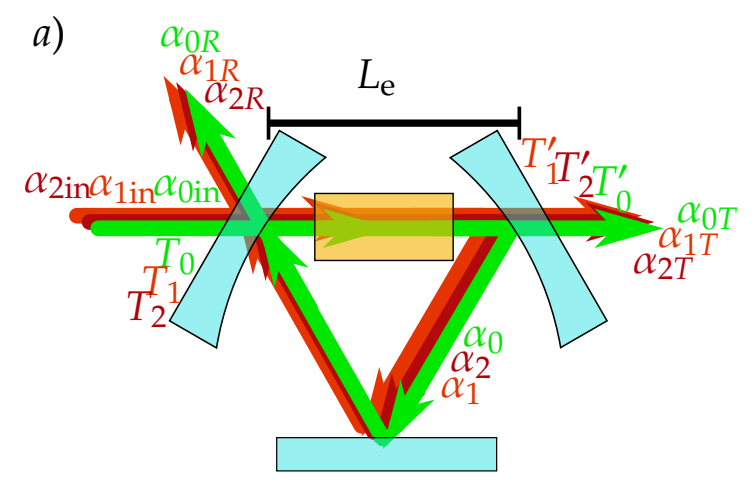

b)

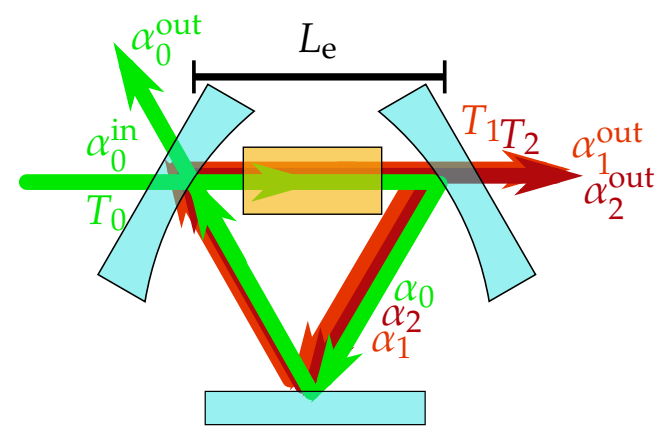

Figura 2.8: Representação da cavidade com o cristal em duas situações. a) Caso geral: a cavidade é bombeada pelos três campos, os quais interagem dentro do cristal. A cavidade é composta por um espelho de acoplamento, com coeficientes de transmissão $T_{i}$, um espelho usado para modelar as perdas intracavidade, com transmissão de $T_{i}^{\prime}$, e um espelho completamente refletor. $b$ ) OPO acima do limiar sem perdas: a cavidade é bombeada somente pelo campo de bombeio. Os feixes gêmeos são produzidos dentro da cavidade e saem pelo espelho de acoplamento. Por conveniência, o espelho de acoplamento é diferente para o bombeio e os feixes gêmeos, como ocorre em grande parte das montagens experimentais.

Considere agora que haja uma cavidade em ane ${ }^{24}$ em torno do cristal de modo que dois dos espelhos possuam coeficientes de transmissão de $T_{j}$ e $T_{j}^{\prime}$ para cada um dos três campos. Ao terminar a volta pelo resto da cavidade cada um dos campos ganha uma fase de $\omega_{j}(L-l) / c$. A cada volta eles são atenuados ao serem refletidos pelos espelhos parcialmente refletores e recebem uma parcela do campo incidente. O campo após uma

\footnotetext{
${ }^{22}$ Esta relação é aproximada, pois, como mostrado em [63, 55, o acordo de fase é proporcional a um seno cardinal. Porém estas contribuições negativas levarão a um menor ganho por passagem pelo cristal.

${ }^{23} \mathrm{O}$ bombeio é atenuado quando há ganho nos feixes gêmeos e vice versa.

${ }^{24}$ Considero aqui uma cavidade em anel no lugar de uma linear por simplicidade. Entretanto, os resultados são essencialmente os mesmo para uma caviade linear.
} 
volta completa pela cavidade é

$$
\overline{\bar{\alpha}}_{j}=r_{j}^{\prime} r_{j} \mathrm{e}^{i \omega_{j}(L-l) / c} \bar{\alpha}_{j}+t_{j} \alpha_{j}^{\text {in }}
$$

onde $r_{j}=\sqrt{R_{j}}, r_{j}^{\prime}=\sqrt{R_{j^{\prime}}^{\prime}}, t_{j}=\sqrt{T_{j}}$ e $t_{j}^{\prime}=\sqrt{T_{j}^{\prime}} \operatorname{com} R_{j}+T_{j}=R_{j}^{\prime}+T_{j}^{\prime}=1$. Da mesma forma que para a cavidade vazia, o campo intracavidade deve se repetir a cada volta após vários ciclos, $\overline{\bar{\alpha}}_{j}=\alpha_{j}$,

$$
\begin{aligned}
& \alpha_{0}\left(1-e^{i \theta_{0}} r_{0} r_{0}^{\prime}\right)=-2 \chi \mathrm{e}^{i \theta_{0}} \alpha_{1} \alpha_{2}+t_{0} \alpha_{0}^{\text {in }}, \\
& \alpha_{1}\left(1-e^{i \theta_{1}} r_{1} r_{1}^{\prime}\right)=2 \chi \mathrm{e}^{i \theta_{1}} \alpha_{0} \alpha_{2}^{*}+t_{1} \alpha_{1}^{\text {in }}, \\
& \alpha_{2}\left(1-e^{i \theta_{2}} r_{2} r_{2}^{\prime}\right)=2 \chi \mathrm{e}^{i \theta_{2}} \alpha_{0} \alpha_{1}^{*}+t_{2} \alpha_{2}^{\text {in }},
\end{aligned}
$$

onde $\theta_{j}=L_{\mathrm{ot}} \omega_{j} / c$, com $L_{\mathrm{ot}}=L+l\left(n_{c}-1\right)$ sendo o comprimento ótico da cavidade. Assim como para a cavidade vazia, a equação $\mathrm{e}^{i \theta_{j}}=1$ define os modos normais da cavidade,

$$
\lambda_{j n}=n L_{\mathrm{ot}}
$$

que são os mesmos para os três campos. Por conveniência, defino as quantidades $\gamma_{j}$, $\mu_{j}$ e $\gamma_{j}^{\prime}=\gamma_{j}+\mu_{j}$ tais que $r_{j}=\mathrm{e}^{-\gamma_{j}}$ e $r_{j}^{\prime}=\mathrm{e}^{-\mu_{j}}$. Na aproximação de alta finesse, $\gamma_{j} \mathrm{e} \mu_{j} \approx 0$ resultando em $r_{j} \approx 1-\gamma_{j}$ e $r_{j}^{\prime} \approx 1-\mu_{j}$. Consequentemente $T_{j} \approx 2 \gamma_{j}$ e $T_{j}^{\prime} \approx 2 \mu_{j}$. Nesta situação, podemos considerar $\theta_{j}$ somente em uma pequena região em torno de uma ressonância,

$$
\mathrm{e}^{i \theta_{j}}=i \delta \theta_{j} \approx 1+i \delta \theta_{j} \equiv 1+i \gamma_{i}^{\prime} \Delta_{j},
$$

pois $\mathrm{e}^{i \theta_{j}}=1$ na ressonância. As quantidades $\Delta_{j}$ são as fases adquiridas em uma volta completa normalizadas pelas perdas e são definidas pela relação (2.36). Partindo da definição de $\theta_{j}$ e de (2.27), a expressão para $\delta \theta_{j}$ pode ser escrita como

$$
\delta \theta_{j}=\left(\omega_{j}-\omega_{j}^{c}\right) \frac{L_{\mathrm{ot}}}{c}=\frac{2 \pi \tilde{\Delta}_{j}}{\mathcal{F}}=2 \widetilde{\Delta}_{j} \gamma_{j}^{\prime},
$$

onde $\omega_{j}^{c}$ é uma ressonância da cavidade e $\widetilde{\Delta}_{j}$ é a dessintonia em unidades da largura de banda para cada campo. Da mesma forma que na seção anterior, $\Delta f$ e $\delta f_{j}$ são, respectivamente, o intervalo espectral livre e a largura de banda da cavidade. Comparando esta última expressão com (2.36) vemos que há um fator 2 entre a fase normalizada pelas perdas e a dessintonia normalizada pela largura de banda, $\Delta_{j}=2 \widetilde{\Delta}_{j}$.

No nosso sistema, somente o campo de bombeio é injetado, consequentemente podemos considerar $\alpha_{1 \text { in }}=\alpha_{2 \text { in }}=0$. Fazendo estas aproximações nas expressões 2.32 
2.34) e mantendo somente os termos dominantes chegamos a

$$
\begin{gathered}
\alpha_{0} \gamma_{0}^{\prime}\left(1-i \Delta_{0}\right)=-2 \chi \alpha_{1} \alpha_{2}+\sqrt{2 \gamma_{0}} \alpha_{0}^{\text {in }}, \\
\alpha_{1} \gamma_{1}^{\prime}\left(1-i \Delta_{1}\right)=2 \chi \alpha_{0} \alpha_{2}^{*}, \quad \alpha_{2} \gamma_{2}^{\prime}\left(1-i \Delta_{2}\right)=2 \chi \alpha_{0} \alpha_{1}^{*} .
\end{gathered}
$$

Neste modelo, $\mu_{j}$ representa a transmissão pelo segundo espelho, mas ela pode modelar qualquer acoplamento da cavidade com o exterior que não ocorra por meio do espelho de acoplamento 25 Por este motivo, $\mu_{j}$ será chamada de perdas espúrias ou perdas intracavidade no decorrer deste texto. Podemos escrever o módulo das equações (2.38) como

$$
\gamma_{1}^{\prime} \sqrt{1+\Delta_{1}^{2}}\left|\alpha_{1}\right|=2 \chi\left|\alpha_{0}\right|\left|\alpha_{2}\right| \quad \text { e } \quad \gamma_{2}^{\prime} \sqrt{1+\Delta_{2}^{2}}\left|\alpha_{2}\right|=2 \chi\left|\alpha_{0} \| \alpha_{1}\right|
$$

Supondo $\left|\alpha_{0}\right| \neq 0,\left|\alpha_{1}\right| \neq 0$ e $\left|\alpha_{2}\right| \neq 0$, as soluções para estas equações são

$$
\gamma_{1}^{\prime} \sqrt{1+\Delta_{1}^{2}}\left|\alpha_{1}\right|^{2}=\gamma_{2}^{\prime} \sqrt{1+\Delta_{2}^{2}}\left|\alpha_{2}\right|^{2} \text { e } \quad\left|\alpha_{0}\right|=\sqrt{\gamma_{1}^{\prime} \gamma_{2}^{\prime} \sqrt{\left(1+\Delta_{1}^{2}\right)\left(1+\Delta_{2}^{2}\right)} / 2 \chi} \text {. }
$$

Se escrevemos (2.38) como

$$
\gamma_{1}^{\prime}\left(1-i \Delta_{1}\right)\left|\alpha_{1}\right| e^{i \phi_{1}}=2 \chi\left|\alpha_{0}\right|\left|\alpha_{2}\right| e^{i\left(\phi_{0}-\phi_{2}\right)} \quad \text { e } \quad \gamma_{2}^{\prime}\left(1-i \Delta_{2}\right)\left|\alpha_{2}\right| e^{i \phi_{2}}=2 \chi\left|\alpha_{0}\right|\left|\alpha_{1}\right| e^{i\left(\phi_{0}-\phi_{1}\right)},
$$

onde $\phi_{j}$ é a fase dos campos intracavidade, a única solução para este novo sistema que é compatível com a encontrada para os módulos é

$$
\Delta_{1}=\Delta_{2}=\Delta, \quad \frac{\left|\alpha_{1}\right|^{2}}{\left|\alpha_{2}\right|^{2}}=\frac{\gamma_{2}^{\prime}}{\gamma_{1}^{\prime}} \quad \text { e } \quad\left|\alpha_{0}\right|^{2}=\frac{\gamma_{1}^{\prime} \gamma_{2}^{\prime}}{4 \chi^{2}}\left(1+\Delta^{2}\right) .
$$

Neste ponto já podemos ver coisas interessantes. Os feixes gêmeos podem estar oscilando em modos normais distintos da cavidade, mas a dessintonia de ambos em relação ao modo da cavidade mais próximo é sempre a mesma. A potência do bombeio intracavidade não depende da potência ou dessintonia do bombeio. Ela é função somente das perdas, da constante de acoplamento $\chi$ e da dessintonia dos feixes gêmeos. Para derivar esta solução é estritamente necessário partir do presuposto que cada um dos três campos possui intensidade não-nula dentro da cavidade. Como discutirei mais adiante, nestas circunstâncias dizemos que o $O P O$ está operando acima do limiar de oscilação. Outra solução possível ocorre abaixo do limiar, quando $\left|\alpha_{1}\right|=\left|\alpha_{2}\right|=0$ e a

\footnotetext{
${ }^{25}$ Nesta categoria podem ser colocadas as perdas por absorção e espalhamento que ocorrem dentro da cavidade.
} 
potência do bombeio intracavidade é proporcional à do campo de entrada,

$$
\alpha_{0}=\frac{\sqrt{2 \gamma_{0}}}{\gamma_{0}^{\prime}\left(1-i \Delta_{0}\right)} \alpha_{0}^{\text {in }}
$$

que é a mesma solução presente em 2.19) e 2.28 para a cavidade vazia. Neste caso, o campo de bombeio intracavidade somente se anula quando $\alpha_{0}^{\text {in }}=0$. À medida que aumentamos a potência do campo de entrada, $\alpha_{0}$ aumenta até alcançar o valor previsto em 2.39) e a cavidade começa a oscilar também para os feixes gêmeos, o que pode ser visto na figura 2.9. A potência do campo de entrada para a qual isto ocorre caracteriza o limiar de oscilação,

$$
\left|\alpha_{0}\right|_{\lim }^{2}=\frac{2 \gamma_{0}}{\left(\gamma_{0}^{\prime}\right)^{2}\left(1+\Delta_{0}^{2}\right)}\left|\alpha_{0}^{\mathrm{in}}\right|_{\lim }^{2}=\frac{\gamma_{1}^{\prime} \gamma_{2}^{\prime}}{4 \chi^{2}}\left(1+\Delta^{2}\right) \Longrightarrow\left|\alpha_{0}^{\mathrm{in}}\right|_{\lim }^{2}=\frac{\gamma_{1}^{\prime} \gamma_{2}^{\prime}\left(\gamma_{0}^{\prime}\right)^{2}}{8 \gamma_{0} \chi^{2}}\left(1+\Delta^{2}\right)\left(1+\Delta_{0}^{2}\right) .
$$
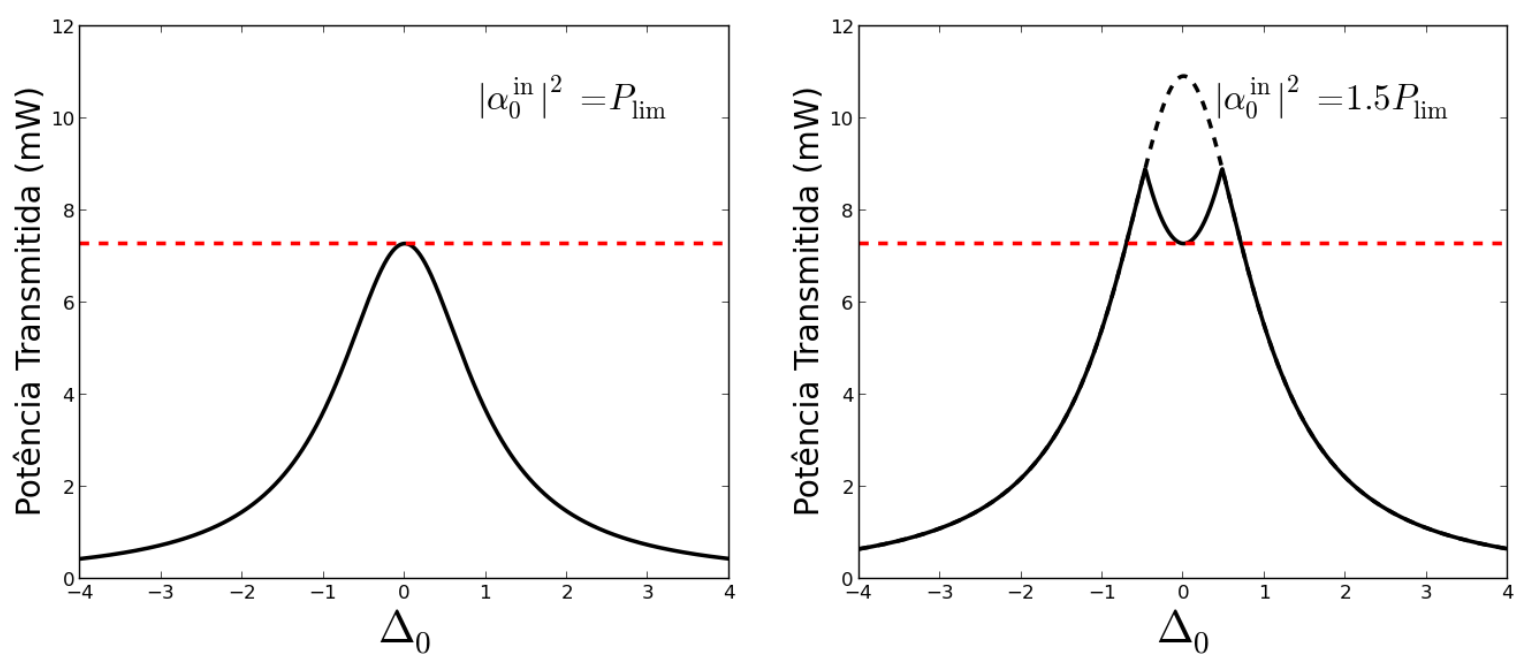

Figura 2.9: Potência de bombeio intracavidade. Na curva da esquerda, o OPO está no limiar de oscilação, abaixo desta potência, o campo transmitido se comporta como em uma cavidade vazia. A curva vermelha representa a potência transmitida exatamente no limiar, $P_{0 T}^{\lim }=T_{0 \max } P_{\lim }$. Na curva da esqerda, o OPO está operando acima do limiar. Podemos ver que a potência transmitida se mantém fixa no valor referente ao limiar. A curva preta tracejada representa a transmissão esperada caso não houvesse a interação não-linear. Nestas curvas foram usados os parâmetros $P_{\lim }=55 \mathrm{~mW}, \gamma_{0}=0.15, \mu_{0}=0.015, b=0.4$ e $\Delta=\Delta_{0}$.

Acima deste limiar, a energia presente nos feixes gêmeos é conseguida às custas da energia do campo de bombeio. Note que o campo de bombeio refletido sofre uma depleção pelo fato de a cavidade possuir perdas, como discutido na seção anterior, que não possui relação com a $P D C$. Porém, devido à presença do cristal, há uma depleção do bombeio adicional devido à $P D C$ como pode ser vista na figura 2.10. Na ressonância 

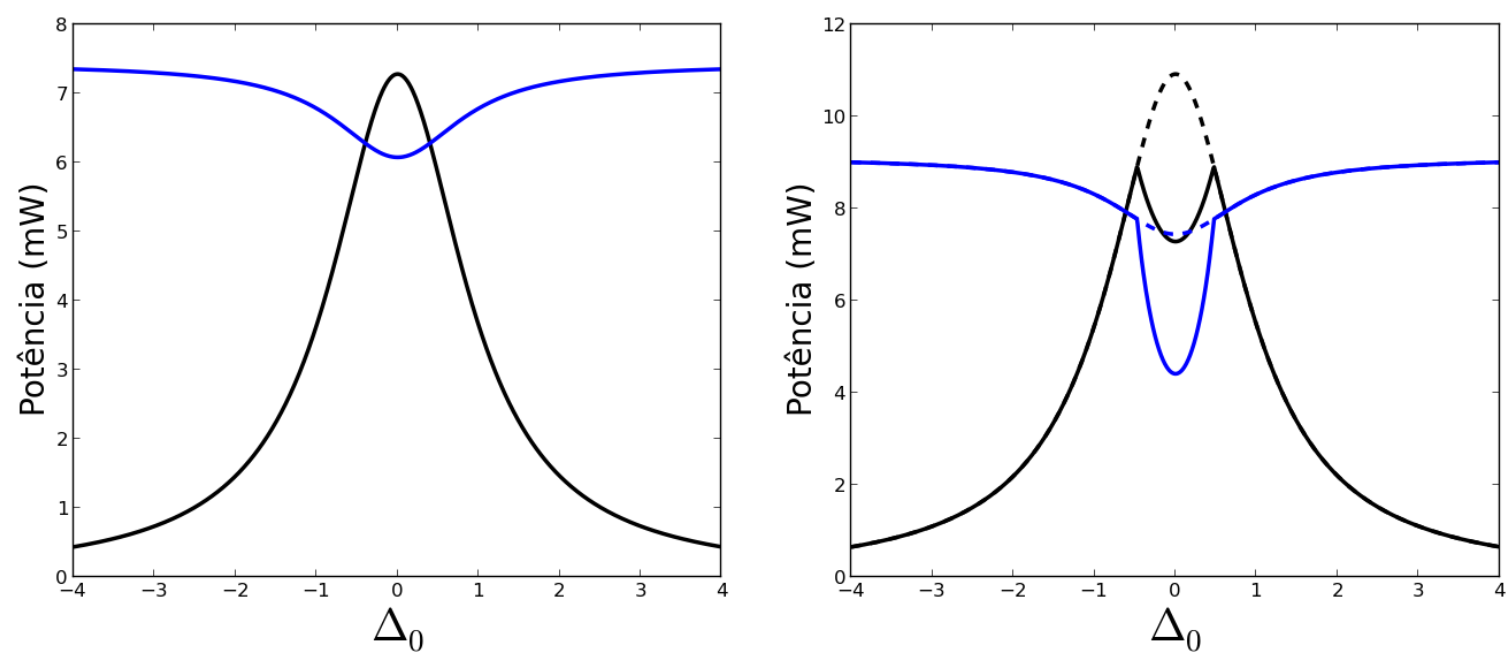

Figura 2.10: As curvas em preto e azul representam, respectivamente, as potências de bombeio transmitida e refletida pela cavidade. A curva da esqueda mostra a depleção do bombeio somente devido à cavidade e a da direita mostra o efeito da coversão do campo de bombeio em sinal e complementar. Nestas curvas foram usados os parâmetros $P_{\lim }=55 \mathrm{~mW}, \gamma_{0}=0.15, \mu_{0}=0.015$, $b=0.4$ e $\Delta=\Delta_{0}$.

para os três campos, o limiar de oscilação chega ao valor mínimo, e esta quantidade é chama de potência de limiar,

$$
P_{\lim }=\frac{\gamma_{1}^{\prime} \gamma_{2}^{\prime}\left(\gamma_{0}^{\prime}\right)^{2}}{8 \gamma_{0} \chi^{2}} .
$$

A partir desta potência do campo de entrada, a potência do bombeio intracavidade se

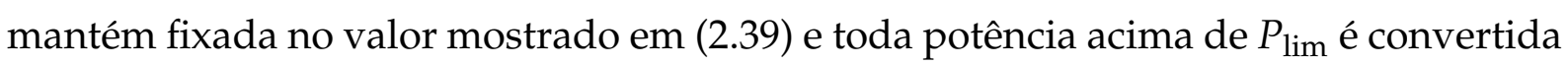
para os feixes gêmeos. Em uma situação não ideal, em que $\mu_{0} \neq 0$, haverá transmissão por outros espelhos que formam a cavidade além da reflexão pelo espelho de acoplamento. Nesta situação, a potência transmitida por um destes outros espelho pode ser escrita em função da potência de limiar como

$$
P_{0 T}=2 \mu_{0} b\left|\alpha_{0}\right|^{2}=T_{0 \max } P_{\lim }\left(1+\Delta^{2}\right),
$$

onde $T_{0 \max }=4 b \mu_{0} \gamma_{0} /\left(\gamma_{0}^{\prime}\right)^{2}$ é a trasmissão em ressonância, como vista em 2.28). O fator $0 \leq b \leq 1$ contabiliza o quanto o espelho em questão contribui para as perdas espúrias. Quando $b=1$, todas as perdas espúrias são devidas a este espelho e quanto menor for $b$, menos este espelho contribui para as mesmas.

Substituindo as relações (2.39) em (2.38) obtemos uma solução para as fases dos 

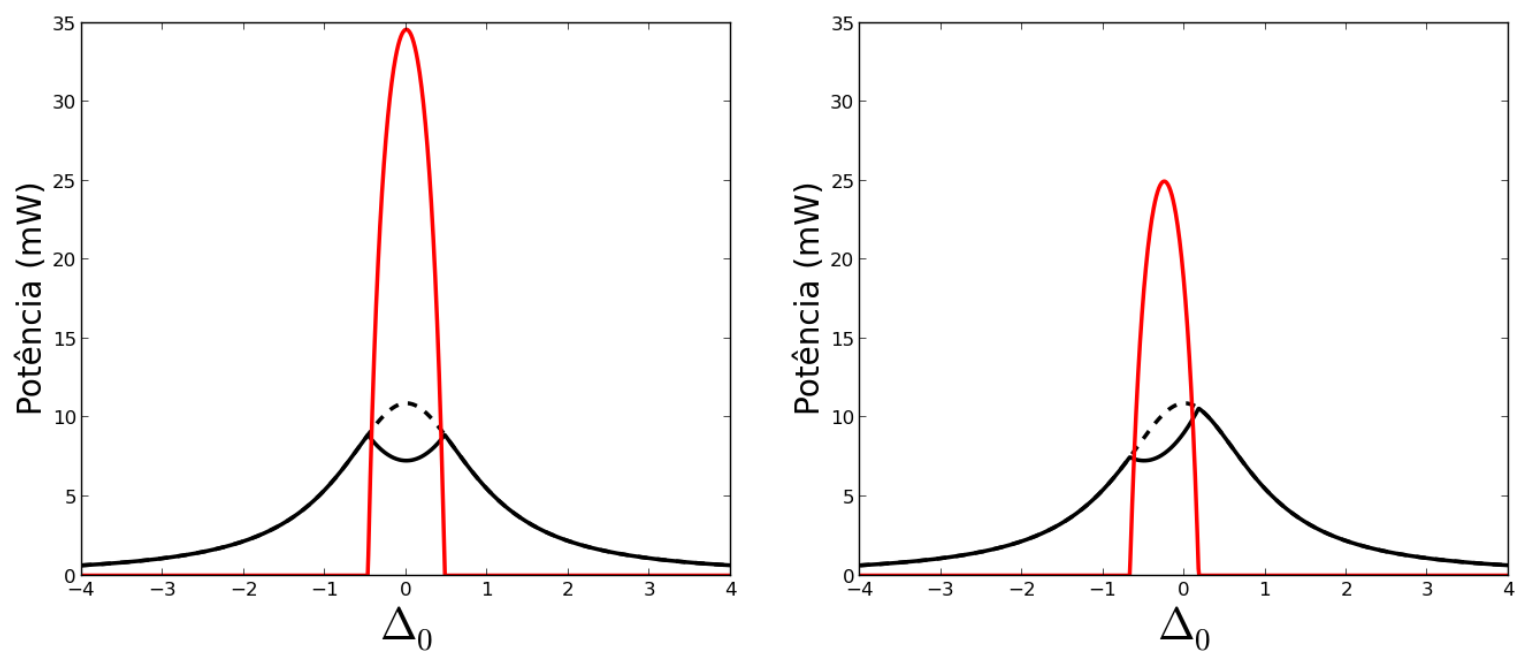

Figura 2.11: Potência transmitida do bombeio (curva preta) e dos feixes gêmeos (curva vermelha). Na figura da esquerda, $\Delta=\Delta_{0}$ e podemos ver o ponto onde o OPO começa a oscilar à medida que variamos a dessintonia da cavidade. Na figura da direita, $\Delta=\Delta_{0}-0.5$. Nesta situação, a eficiência de conversão é menor, o que pode ser visto pela diminuição da potência máxima alcançada pelos feixes gêmeos. Quando variamos o tamanho da cavidade, tanto $\Delta$ quanto $\Delta_{0}$ são modificadas. Porém é possivel ajustar a diferença entre ambas variando a a ângulação do cristal e/ou a sua temperatura. Estas curvas foram traçadas com os seguintes parâmetros: $P_{\lim }=55 \mathrm{~mW}, \gamma_{0}=0.15, \mu_{0}=0.015, \gamma=0.02, \mu=0.006$ e $b=0.4$.

campos intracavidade,

$$
\mathrm{e}^{i\left(\phi_{0}-\phi_{1}-\phi_{2}\right)}=\frac{(1-i \Delta)}{\sqrt{1+\Delta^{2}}},
$$

o que significa que a soma das fases dos feixes gêmeos está perfeitamente correlacionada com a fase do bombeio e que quando a cavidade está em ressonância $\phi_{0}=\phi_{1}+\phi_{2}$. Cada uma das fases $\phi_{j}$ varia à medida que os três campos se propagam pela cavidade, porém, devido ao acordo de fases, a relação (2.44) se mantém constante em todos os pontos. Substituindo esses valores na equação (2.37) obtemos

$$
\gamma_{0}^{\prime}\left(1-i \Delta_{0}\right)\left|\alpha_{0}\right|+\left|\alpha_{1}\right|\left|\alpha_{2}\right| \frac{(1-i \Delta)}{\sqrt{1+\Delta^{2}}}=\sqrt{2 \gamma_{0}}\left|\alpha_{0}^{\mathrm{in}}\right| e^{i\left(\phi_{i n}-\phi_{0}\right)},
$$

cuja solução para o módulo, usando (2.39), é

$$
\left|\alpha_{1}\right|\left|\alpha_{2}\right|=\frac{2 \gamma_{0} P_{\lim }}{\sqrt{\gamma_{1}^{\prime} \gamma_{2}^{\prime}} \gamma_{0}^{\prime}}\left[\Delta \Delta_{0}-1+\sqrt{\sigma-\left(\Delta+\Delta_{0}\right)^{2}}\right],
$$

onde $\sigma=\left|\alpha_{0}^{\mathrm{in}}\right|^{2} / P_{\text {lim }}$ é a potência de entrada em unidades de potência de limiar. A variação da potência com a dessintonia pode ser vista na figura 2.11 .

Como no nosso sistema os feixes gêmeos tem aproximadamente a mesma frequência, podemos considerar que tanto o coeficiente de transmissão do espelho de acoplamento 
quanto as perdas espúrias sejam iguais para ambos, $\gamma_{1}=\gamma_{2}=\gamma$ e $\mu_{1}=\mu_{2}=\mu$, consequentemente $\left|\alpha_{1}\right|=\left|\alpha_{2}\right|=|\alpha|$. Desta forma, a potência dos feixes gêmeos intracavidade é dada pela expressão

$$
|\alpha|^{2}=\frac{2 \gamma_{0} P_{\lim }}{\gamma^{\prime} \gamma_{0}^{\prime}}\left[\Delta \Delta_{0}-1+\sqrt{\sigma-\left(\Delta+\Delta_{0}\right)^{2}}\right],
$$

Note que a intensidade é medida na mesma unidade em que $P_{\text {lim }}$, pois todas as outras quantidades envolvidas são adimensionais. Entretanto, o campo que temos acesso no experimento é o transmitido pela cavidade que é igual a

$$
P_{T}=2 \gamma|\alpha|^{2}=4 \eta_{\mathrm{OPO}} P_{\lim }\left[\Delta \Delta_{0}-1+\sqrt{\sigma-\left(\Delta+\Delta_{0}\right)^{2}}\right]
$$

onde $\eta_{\mathrm{OPO}}=\gamma \gamma_{0} / \gamma_{0}^{\prime} \gamma^{\prime}$ é a eficiência quântica total de conversão do OPO. Ela é a multiplicação das eficiências do bombeio e dos feixes gêmeos, as quais medem a fração do campo intracavidade transmitida pelo espelho de acoplamento. 


\section{Métodos de Medida das Flutuações da Luz}

Neste capítulo, descrevo em detalhes os métodos que utilizamos para caracterizar os estados que preparamos no laboratório. Esses se estendem desde o processo de absorção pelo fotodiodo até o papel das cavidades de análise e da demodulação do sinal eletrônico obtido a partir do fotodetector. Ele é um dos capítulos centrais da tese e me esforcei para fazer um discussão tão simples quanto possível sem que a essência dos conceitos físicos tratados fosse sacrificada.

A primeira parte da discussão que farei e os conceitos e resultados apresentados já são conhecidos na literatura, porém de forma difusa e mais intrincada. Vou introduzilos aqui com dois objetivos, o primeiro de apresentar uma abordagem mais simples e próxima do nosso entendimento atual e o segundo de fixar a notação e preparar para as discussões subsequentes. A segunda parte, que compõem as últimas seções, são parte de um trabalho original [66]. A abordagem teórica aqui empregada é baseada em [62, 67, 68, 69, 70].

\subsection{Fotodetecção de Feixes Intensos e Monocromáticos}

Em nosso experimento usamos fotodiodos para detectar os feixes de luz que produzimos. Estes são sensíveis somente à intensidade, pois são baseados no efeito fotoelétrico. A intensidade média medida por eles durante o intervalo de medida, $\Delta t$, é proporcional à função de correlação de Glauber integrada durante este intervalo [71, 72, 62],

$$
\langle I(t)\rangle=S_{N} \int_{t}^{t+\Delta t}\left\langle\hat{E}^{-}(r, t) \hat{E}^{+}(r, t)\right\rangle d t,
$$

onde $r$ é a posição do detetor e $S_{N}$ é uma constante que depende dos parâmetros do fotodiodo, associada à eficiência quântica do detetor, e o símbolo \langle\rangle detona a média 
sobre um conjunto de medidas desta mesma quantidade $\mathrm{e}^{1}$. O operador campo elétrico é dado por

$$
\begin{gathered}
\hat{E}(r, t)=\hat{E}^{+}(r, t)+\hat{E}^{-}(r, t), \\
\hat{E}^{+}(r, t)=\sum_{j} g_{j} \hat{a}_{j} \mathrm{e}^{i\left(k_{j} r-\omega_{j} t\right)}, \quad \text { com } g_{j}=i \sqrt{\frac{\hbar \omega_{j}}{2 \epsilon_{0} V}},
\end{gathered}
$$

onde $\hat{E}^{-}(r, t)=\left[\hat{E}^{+}(r, t)\right]^{\dagger}, \hat{a}_{j}$ é o operador de aniquilação de fótons na frequência $\omega_{j} \geq 0$ e $V$ é o volume de quantização e $g_{j}$ é o campo elétrico por fóton [73, 70]. A média em (3.1) é tomada sobre o estado inicial do campo, $\hat{\rho}$.

A intensidade luminosa será convertida em uma corrente elétrica no fotodiodo, chamada fotocorrente. Consequentemente, a fotocorrente média também é proporcional à função de correlação de Glauber integrada do tempo de medida. A partir deste ponto, vou considerar o intervalor da medida bastante curto de tal forma que podemos retirar a integração ${ }^{2}$

$$
\langle i(t)\rangle=K\left\langle\hat{E}^{-}(r, t) \hat{E}^{+}(r, t)\right\rangle,
$$

onde $K$ é uma constante que captura tanto $S_{N}$ quanto qualquer amplificação introduzida pela eletrônica de detecção. A expressão (3.4) coincide com a intensidade média da eletrodinâmica clássica quando os operadores $\hat{E}^{ \pm}$são substituídos por números complexos.

Os estados aos quais temos acesso no laboratório são formados por um conjunto de modos densamente populado - com frequências em torno de uma frequência central, chamada portadora - e por suas bandas laterais com valor médio nulo e formadas por todas frequências fora desta banda central. De início, considere que não há correlações entre a portadora e as bandas laterais,

$$
\hat{\rho}=\hat{\rho}_{p} \otimes \hat{\rho}_{\mathrm{BL}}
$$

Esta suposição não é de fato necessária, pois o tipo de medida que realizamos é insensível a qualquer correlação entre portadora e bandas laterais. Como as correlações não são importantes neste contexto, podemos considerá-las nulas sem perda de generalidade. Como será explicitado mais adiante, a portadora é utilizada para amplificar as propriedades das bandas laterais sendo, portanto, parte do aparato de medida. De

\footnotetext{
${ }^{1}$ média sobre ensemble.

${ }^{2}$ No decorrer deste e do próximo capítulo, vou associar este intervalo de integração à largura de banda, ou precisão temporal, da nossa eletrônica de detecção. Para as considerações feitas no momento, não é necessário manter a integração.
} 


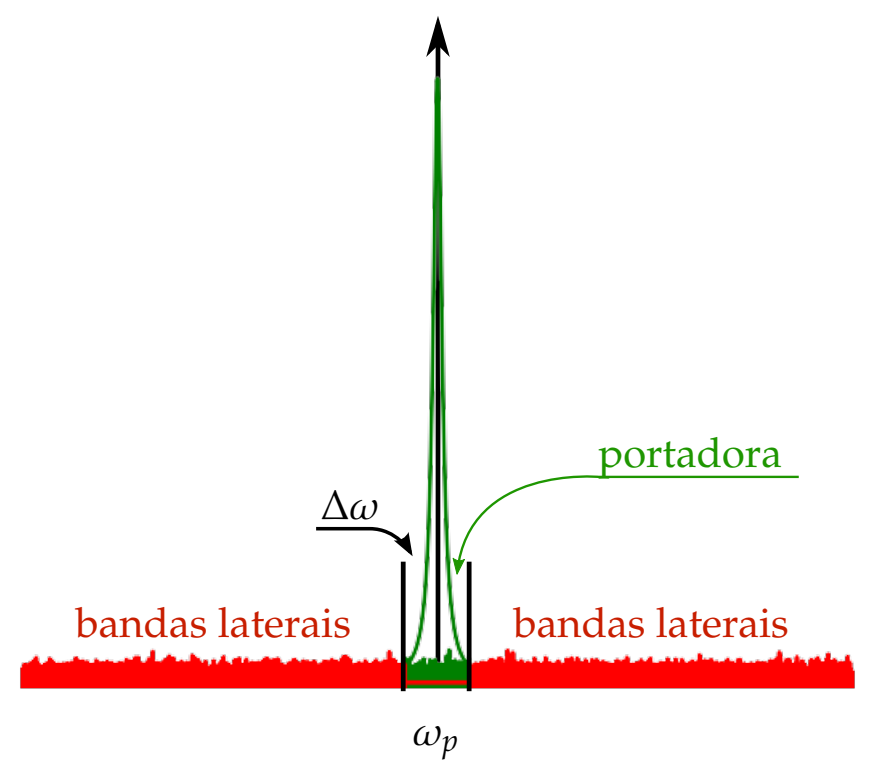

Figura 3.1: Espectro de intensidade de um laser. A curva lorentziana verde representa o campo clássico e o ruído em vermelho representa as flutuações do vácuo.

fato, os estados nos quais estamos interessados fazem parte somente de $\hat{\rho}_{\mathrm{BL}}$. Os estados $\hat{\rho}_{p}$ e $\hat{\rho}_{\mathrm{BL}}$ são definidos por um corte em torno da frequência da portadora de modo que $\hat{\rho}_{p}$ e $\hat{\rho}_{\text {BL }}$ são operadores que atuam, respectivamente, nos espaços de Hilbert

$$
\mathcal{H}_{p}=\bigotimes_{\omega_{i} \in \delta \omega} \mathcal{H}_{i} \quad \text { e } \quad \mathcal{H}_{\mathrm{BL}}=\bigotimes_{\omega_{i} \notin \delta \omega} \mathcal{H}_{i}
$$

onde $\mathcal{H}_{i}$ são os espaços de Hilbert de cada modo de frequência $\omega_{i}$ e a notação $\omega_{i} \in \delta \omega$ significa que $\omega_{i}$ está dentro do intervalo $\omega_{p}-\Delta \omega \leq \omega_{i} \leq \omega_{p}+\Delta \omega$. A frequência de corte, $\Delta \omega$, dever ser maior que a largura de linha do feixe e é típica de cada detector. Em nosso experimento, a largura de linha do laser fornecida pelo fabricante é de $1 \mathrm{kHz}$. A frequência que usamos para separar a portadora das bandas laterais é de $300 \mathrm{kHz}$. Como esta frequência é bem maior que a largura de linha, não é possível confundir as bandas laterais com a portadora.

Com esta motivação, o operador de campo é separado em duas componentes: uma que representa a portadora e outra que representa as bandas laterais

$$
\begin{gathered}
\hat{E}^{+}(t)=\hat{E}_{p}^{+}(t)+\hat{E}_{\mathrm{BL}}^{+}(t), \\
\hat{E}_{p}^{+}(t)=g \sum_{\omega_{i} \in \delta \omega} \hat{a}_{\omega_{i}} \mathrm{e}^{-i \omega_{i} t}, \quad \hat{E}_{\mathrm{BL}}^{+}(t)=g \sum_{\omega_{i} \notin \delta \omega} \hat{a}_{\omega_{i}} \mathrm{e}^{-i \omega_{i} t},
\end{gathered}
$$


pois os detetores possuem uma largura de banda tal que $g_{j}=i \sqrt{\frac{\hbar \omega_{j}}{2 \epsilon_{0} V}} \approx i \sqrt{\frac{\hbar \omega_{p}}{2 \epsilon_{0} V}}=g$, onde $\omega_{p}$ é a frequência da portadora e também considero que estamos no referencial do detetor, $r=0$. A componente $\hat{E}_{\mathrm{BL}}^{ \pm}$somente atua em $\mathcal{H}_{\mathrm{BL}}$ e $\hat{E}_{p}^{ \pm}$em $\mathcal{H}_{p}$. Por simplicidade, considero a portadora formada por apenas um modo e em um estado coerente [74]

$$
\hat{\rho}_{p}=\left|\alpha_{p}\right\rangle\left\langle\alpha_{p}\right|, \text { tal que } \hat{a}_{\omega_{p}}\left|\alpha_{p}\right\rangle=\alpha_{p}\left|\alpha_{p}\right\rangle
$$

Toda a contribuição para o valor médio da intensidade é somente devida à portadora, calculada no estado $\rho_{p}$. Substituindo (3.8) 3.9) explicitamente em (3.4), chegamos em uma intensidade média de

$$
\langle i(t)\rangle=K\left\langle\hat{E}^{-}(t) \hat{E}^{+}(t)\right\rangle=K|g|^{2}\left|\alpha_{p}\right|^{2}+K g\left|\alpha_{p}\right|\left\langle\mathrm{e}^{-i \phi_{p}} \hat{E}_{\mathrm{BL}}^{+}(t)+\mathrm{e}^{i \phi_{p}} \hat{E}_{\mathrm{BL}}^{-}(t)\right\rangle_{\mathrm{BL}}=K|g|^{2}\left|\alpha_{p}\right|^{2},
$$

pois $\left\langle\hat{E}_{0}^{ \pm}(t)\right\rangle=0, \alpha_{p}=\left|\alpha_{p}\right| \mathrm{e}^{-i \phi_{p}}$ e desprezamos o termo referente ao batimento das bandas laterais com elas mesmas, $\left\langle\hat{E}_{\mathrm{BL}}^{-}(t) \hat{E}_{\mathrm{BL}}^{+}(t)\right\rangle_{\mathrm{BL}}=\operatorname{Tr}\left[\hat{E}_{\mathrm{BL}}^{-}(t) \hat{E}_{\mathrm{BL}}^{+}(t) \hat{\rho}_{\mathrm{BL}}\right]$, e que representa a intensidade média nas bandas laterais. Consequentemente, $\langle i(t)\rangle$ é resultado somente da intensidade média da portadora que, como $K$ depende apenas do sistema de detecção e $g$ da frequência, é ajustada no experimento por meio de $\left|\alpha_{p}\right|^{2}$.

Como a relação (3.10) deve ser válida para qualquer estado, associamos o operador dentro da média,

$$
\delta \hat{i}(t)=K\left|\alpha_{p}\right| g\left[\mathrm{e}^{-i \phi_{p}} \hat{E}_{\mathrm{BL}}^{+}(t)+\mathrm{e}^{i \phi_{p}} \hat{E}_{\mathrm{BL}}^{-}(t)\right]=K\left|\alpha_{p} \| g\right|^{2} \delta \hat{q}_{\phi_{p}}(t),
$$

com o operador de medida nas bandas laterais, que representa a quantidade física que estamos observando diretamente na saída do fotodiodo. Como se trata de um observável físico, $\delta i$ é hermitiano. Ele também possui média nula, e se torna proporcional a um operador de quadratura formado pela integração dos modos nas bandas laterais,

$$
\delta \hat{q}_{\phi_{p}}(t)=\mathrm{e}^{-i \phi_{p}} \delta \hat{a}(t)+\mathrm{e}^{i \phi_{p}} \delta \hat{a}^{\dagger}(t) \quad \text { e } \delta \hat{a}(t)=\int_{\Omega>0}^{+\infty} d \Omega\left[\hat{a}_{\Omega} \mathrm{e}^{-i \Omega t}+\hat{a}_{-\Omega} \mathrm{e}^{i \Omega t}\right],
$$

onde $\Omega=\omega-\omega_{p}$, com $\omega$ sendo o limite contínuo de $\omega_{i}$. Note que a flutuação na fotocorrente é uma soma de sinais que oscilam nas frequências de batimento entre cada banda lateral e a portadora. Como pode ser visto em (3.11), as flutuações na fotocorrente são proporcionais a multiplicação de $\delta \hat{q}_{\phi_{p}}$ por $\left|\alpha_{p}\right|$. Isto significa que a informação contida nas bandas laterais é amplificada pela portadora. O operador $\delta \hat{a}(t)$ cria fótons nas bandas laterais no tempo $t$, suas relações de comutação são derivadas de (3.12),

$$
\left[\delta \hat{a}(t), \delta \hat{a}^{\dagger}\left(t^{\prime}\right)\right]=2 \pi \delta\left(t-t^{\prime}\right),
$$


e ele representa um modo do campo resolvido no tempo com valor médio nulo. Os autovalores do operador $\hat{n}(t)=\delta \hat{a}^{\dagger}(t) \delta \hat{a}(t)$ são o número de fótons criados no tempo $t$ nas bandas laterais por unidade de tempo e representa diretamente o fluxo de fótons total nas bandas laterais.

O operador $\delta \hat{q}_{\phi_{p}}(t)$ pode ser escrito como uma transformada de Fourier de um operador não-hermitiano composto por ambas as bandas laterais superior e inferior,

$$
\delta \hat{q}_{\phi_{p}}(t)=\int_{-\infty}^{+\infty} d \Omega \delta \hat{q}_{\phi_{p}}(\Omega) \mathrm{e}^{-i \Omega t}, \quad \text { onde } \quad \delta \hat{q}_{\phi_{p}}(\Omega)=\cos \phi_{p}\left[\frac{\hat{q}_{+}+i \hat{p}_{-}}{\sqrt{2}}\right]+\operatorname{sen} \phi_{p}\left[\frac{\hat{p}_{+}-i \hat{q}_{-}}{\sqrt{2}}\right]
$$

e que será chamado de operador de quadratura espectral. Note que o operador $\delta \hat{q}_{\phi_{p}}(\Omega)=$ $\left[\delta \hat{q}_{\phi_{p}}(-\Omega)\right]^{\dagger}$ não pode ser associado a um observável físico, pois não é hermitiano. Ele é composto pelos operadores de soma e subtração de bandas laterais, $\hat{q}_{ \pm}$e $\hat{p}_{ \pm}$que são observáveis físicos e são combinações dos operadores de quadratura que atuam nas bandas laterais superior e inferior,

$$
\hat{q}_{ \pm}=\hat{a}_{ \pm}+\hat{a}_{ \pm}^{\dagger}, \quad \text { e } \quad \hat{p}_{ \pm}=i\left(\hat{a}_{ \pm \Omega}^{\dagger}-\hat{a}_{ \pm \Omega}\right) \quad \text { com } \quad \hat{a}_{ \pm}=\frac{\hat{a}_{\Omega} \pm \hat{a}_{-\Omega}}{\sqrt{2}}
$$

Devido à mistura das bandas laterais inferior e superior no fotodetector, o operador
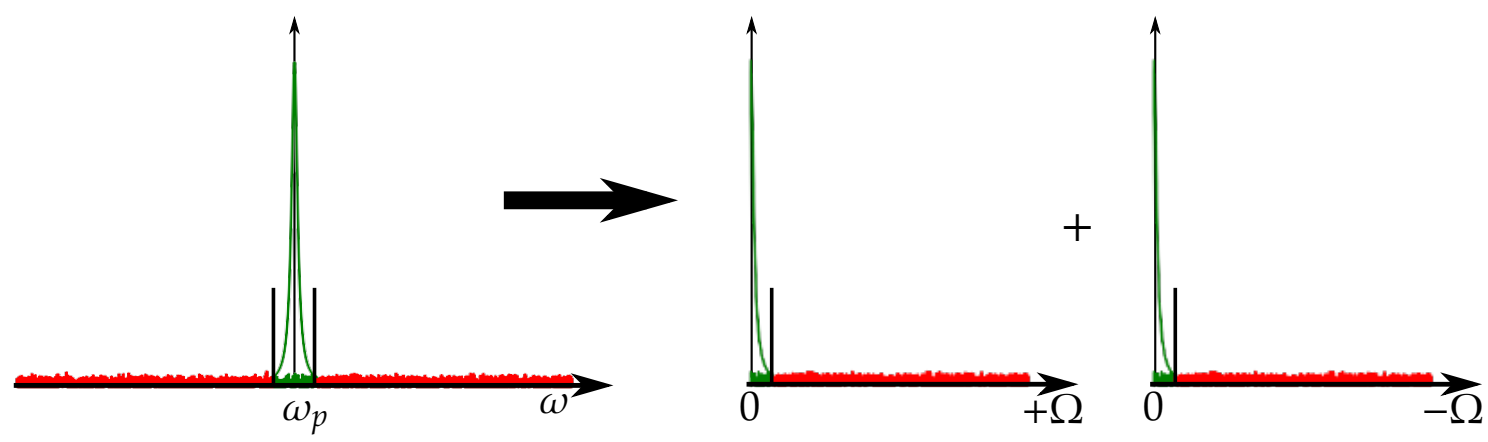

Figura 3.2: A componente de alta frequência do sinal de saída do fotodetector é proporcional ao batimento entre portadora e bandas laterais. Por isso o sinal eletrônico de frequência $\Omega$ é formado pela mistura das bandas laterais de frequência $\omega_{p}-\Omega$ e $\omega_{p}+\Omega$, como mostrado em 3.143 .15 .

de quadratura espectral na frequência $\Omega$ depende de ambas, como fica explícito em (3.14) e (3.15). Esta mistura acontece de modo a favorecer os operadores de soma e subtração de bandas laterais. Isto se parece com a medida na base de Bell em variáveis discretas, onde estados coletivos e emaranhados de dois qbits são usados como base para a medida ${ }^{3}$ Uma representação dessa medida é mostrada na figura 3.3 . No

\footnotetext{
${ }^{3} \mathrm{Ou}$ em contrapartida operadores coletivos no formalismo de Heisenberg.
} 
espaço de subtração, os papéis das quadraturas fase e amplitude estão trocados, o que é equivalente a dizer que há uma fase de $\pi / 2$ entre os espaços de soma e subtração. Um forma direta de compreender isto é fazer a transformação $\hat{a}_{-} \rightarrow i \hat{a}_{-}$. Ela faz com que os operadores de quadratura se tornem $\hat{q}_{-} \rightarrow-\hat{p}_{-}$e $\hat{p}_{-} \rightarrow \hat{q}_{-}$, que é uma das mais simples transformações canônicas [75, 76]

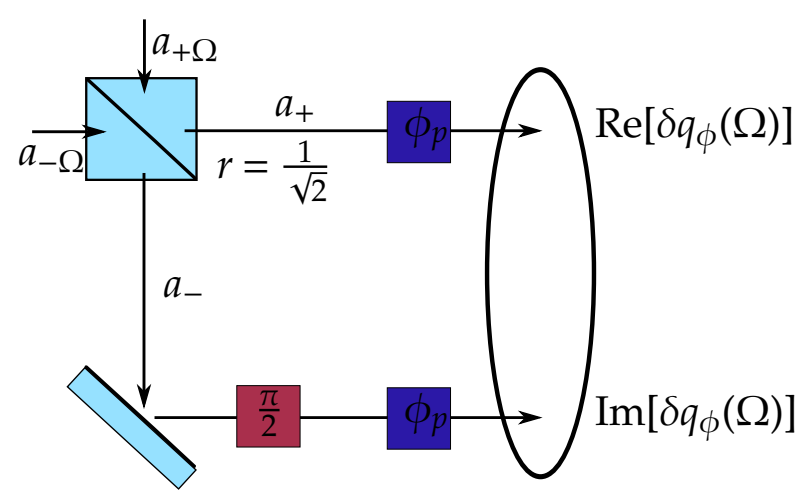

Figura 3.3: Representação pictórica da medida realizada pelo fotodetector em cada componente de frequência, $\delta \hat{q}_{\phi}(\Omega)$, pode ser visualizada como uma transformação de divisor de feixes em que uma das saídas recebe uma fase de $\pi / 2$. Mais à frente será mostrado como é possível controlar a fase $\phi_{p}$ por meio da detecção homodina.

Tendo como motivação (3.11) e (3.10), podemos definir o operador intensidade, que representa a medida direta da fotocorrente, como

$$
\hat{I}(t)=\bar{I}+\delta \hat{I}(t), \quad \text { com } \quad \bar{I}=\langle i(t)\rangle=K|g|^{2}\left|\alpha_{p}\right|^{2} \quad \text { e } \quad \delta \hat{I}(t)=\delta \hat{i}(t)=\sqrt{K}|g| \sqrt{\bar{I}} \delta \hat{q}_{\phi_{p}}(t) .
$$

O qual é associado ao modo temporal,

$$
\hat{I}(t)=K|g|^{2}\left[\left|\alpha_{p}\right|^{2}+\alpha_{p} \delta \hat{a}^{\dagger}(t)+\alpha_{p}^{*} \delta \hat{a}(t)\right] \approx K|g|^{2} \hat{a}^{\dagger}(t) \hat{a}(t)
$$

onde $\hat{a}(t)=\alpha_{p}+\delta \hat{a}(t)$ é um operador efetivo que contém a informação sobre portadora e bandas laterais. A aproximação em 3.17) é válida quando $\delta \hat{a}^{\dagger} \delta \hat{a}$ é desprezível. O que significa dizer que definir o operador efetivo $\hat{a}(t)$ somente faz sentido quando o fluxo de fótons nas bandas laterais for muito menor que a intensidade da portadora. Nos estados que produzimos, $\left\langle\delta \hat{a}^{\dagger} \delta \hat{a}\right\rangle \sim 1$, enquanto $\left|\alpha_{p}\right|^{2} \sim 10^{16}$.

Em um campo real, a diferença de fase $\phi_{p}$ entre a portadora e as bandas laterais se anula, pois ambas são criadas ao mesmo tempo e se propagam juntas pela mesma distância. A defasagem devida à diferença de frequências é capturada pela função exponencial em (3.14) e irá ser convertida para a fotocorrente. No contexto experimental, é possível introduzir um diferença de fase controlável de diversas maneiras. Alguns 
exemplos são a detecção homodina [77, 78, 79], a detecção com cavidades óticas [80, 81] e a separação entre bandas laterais em um interferômetro de Mach-Zehnder bastante assimétrico [82]. Como usamos a detecção com cavidades e a detecção homodina em nossos experimentos, ambas técnicas serão explicadas em detalhes do decorrer do capítulo. Tendo isso em mente, a medida direta em um fotodiodo fornece somente a flutuação na quadratura das bandas laterais que está em fase com a amplitude do campo médio da portadora,

$$
\delta \hat{q}(t) \equiv \delta \hat{q}_{0}(t)=\delta \hat{a}(t)+\delta \hat{a}^{\dagger}(t)
$$

O que é razoável, pois os fotodiodos, por medirem a intensidade do campo, são insensíveis à sua fase.

\subsection{Detecção Homodina}

A detecção homodina consiste em misturar em um divisor de feixes o campo cujas flutuações se deseja medir com um campo de referência bem conhecido, chamado oscilador local ótico, e preferencialmente em um estado coerente. A intensidade dos campos de saída do divisor de feixes irá depender da fase relativa entre ambos devido à interferência entre os campos de entrada. Esta mesma dependência na fase relativa aparece também nas flutuações, de tal modo que é possível medir a quantidade mostrada em 3.14 indiretamente, mas agora com $\phi_{p}$ representando a fase relativa entre os campos de entrada. Pelo fato da medirmos as flutuações na frequência de batimento entre as bandas laterais e a portadora, ruídos clássicos de baixa frequência podem se tornar importantes neste tipo de medida. Para contornar este problema, foi proposto usar divisores de feixes balanceados e medir a informação contida na subtração das intensidades de cada saída do detector [83, 84]. Esse procedimento isola somente os termos de interferência tanto na intensidade média quanto nas flutuações. Após a mistura dos modos temporais $\hat{a}$ e $\hat{a}_{\mathrm{LO}}{ }^{4}$ que representam, respectivamente, o sistema que desejamos medir e o oscilador local, os modos de saída são

$$
\hat{a}_{1}=\frac{\hat{a}+\hat{a}_{\mathrm{LO}}}{\sqrt{2}} \quad \text { e } \quad \hat{a}_{2}=\frac{\hat{a}-\hat{a}_{\mathrm{LO}}}{\sqrt{2}} .
$$

A descrição do processo de medição desenvolvida na seção anterior é válida para ambos os campos de saída do divisor de feixes, os quais estão relacionados com os

\footnotetext{
${ }^{4}$ Aqui oculto a dependência temporal para simplificar a notação.
} 


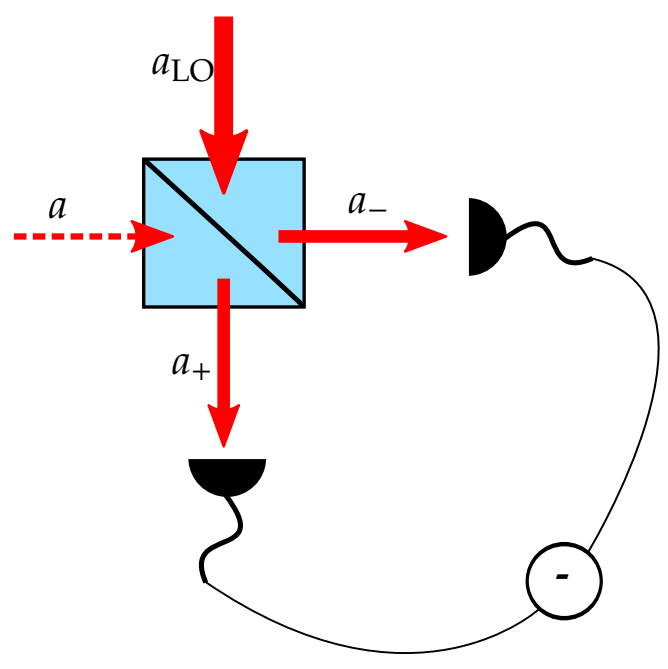

Figura 3.4: Diagrama representando a deteção homodina. No caso em que divisor de feixes possui coeficientes de transmissão e reflexão iguais recuperamos a detecção homodina balanceada.

campos que entrada por meio das transformações (3.19). Os estados de ambos também se separam em portadora e bandas laterais,

$$
\hat{a}(t)=\alpha+\delta \hat{a} \quad \text { e } \quad \hat{a}_{\mathrm{LO}}=\alpha_{\mathrm{LO}}+\delta \hat{a}_{\mathrm{LO}}
$$

de modo que não há correlações entre as bandas laterais de ambos, $\left\langle\delta \hat{a}_{\mathrm{LO}} \delta \hat{a}\right\rangle=\left\langle\delta \hat{a}_{\mathrm{LO}}^{\dagger} \delta \hat{a}\right\rangle=$ 0 . Os operadores intensidade como definidos em (3.17) são

$$
\hat{I}_{1}=\hat{a}_{1}^{\dagger} \hat{a}_{1}=\frac{1}{2}\left[\hat{I}+\hat{I}_{\mathrm{LO}}+\hat{a}_{\mathrm{LO}}^{\dagger} a+\hat{a}^{\dagger} \hat{a}_{\mathrm{LO}}\right] \text { e } \quad \hat{I}_{2}=\hat{a}_{2}^{\dagger} \hat{a}_{2}=\frac{1}{2}\left[\hat{I}+\hat{I}_{\mathrm{LO}}-\hat{a}_{\mathrm{LO}}^{\dagger} \hat{a}-\hat{a}^{\dagger} \hat{a}_{\mathrm{LO}}\right],
$$

onde $\hat{I}=\hat{a}^{\dagger} \hat{a}$ e $\hat{I}_{\mathrm{LO}}=\hat{a}_{\mathrm{LO}}^{\dagger} \hat{a}_{\mathrm{LO}}$. Portanto, as intensidades médias dos campos são

$$
\bar{I}_{1}=\left\langle\hat{a}_{1}^{\dagger} \hat{a}_{1}\right\rangle=\frac{\bar{I}+\bar{I}_{\mathrm{LO}}}{2}+\sqrt{\bar{I}_{\mathrm{LO}} \bar{I}} \cos \phi \quad \text { e } \quad \bar{I}_{2}=\left\langle\hat{a}_{2}^{\dagger} \hat{a}_{2}\right\rangle=\frac{\bar{I}+\bar{I}_{\mathrm{LO}}}{2}-\sqrt{\bar{I}_{\mathrm{LO}} \bar{I}} \cos \phi,
$$

onde $\bar{I}=\left\langle\hat{a}^{\dagger} \hat{a}\right\rangle, \bar{I}_{\mathrm{LO}}=\left\langle\hat{a}_{\mathrm{LO}}^{\dagger} \hat{a}_{\mathrm{LO}}\right\rangle$ e $\phi$ é a fase relativa entre o o campo a ser medido e o oscilador local. Note que a subtração das intensidades médias, $\bar{I}_{-}=\left\langle\hat{I}_{1}-\hat{I}_{2}\right\rangle$, é proporcional ao termo de interferência. Usando (3.20), o operador intensidade para a subtração das fotocorrentes é

$$
\hat{I}_{-}=\hat{a}_{\mathrm{LO}}^{\dagger} \hat{a}+\hat{a}^{\dagger} \hat{a}_{\mathrm{LO}}=\bar{I}_{-}+\delta \hat{I}_{-}, \quad \text { onde } \quad \delta \hat{I}_{-}(t)=\sqrt{K}|g|\left[\left|\alpha_{\mathrm{LO}}\right| \delta \hat{q}_{\phi}(t)+|\alpha| \delta \hat{q}_{-\phi}^{\mathrm{LO}}(t)\right],
$$

onde $\delta \hat{q}_{\phi}(t)$ e $\delta \hat{q}_{-\phi}^{\mathrm{LO}}(t)$ são definidos como em 3.14 . Como a defasagem introduzida na detecção homodina é independente da frequência das bandas laterais, ela leva a um operador de medida como o mostrado em (3.14). Diferente do que ocorre com a fase entre portadora e bandas laterais do mesmo campo, a fase $\phi$ pode ser controlada com 
facilidade.

O ruído na subtração das intensidades é a mistura dos ruídos de ambos os campos ponderados pelas intensidades médias do outro,

$$
\Delta^{2} \hat{I}_{-}=\left\langle\left(\delta \hat{I}_{-}\right)^{2}\right\rangle=K|g|^{2}\left[\bar{I}_{\mathrm{LO}} \Delta^{2} \hat{q}_{\phi}(t)+\bar{I} \Delta^{2} \hat{q}_{-\phi}^{\mathrm{LO}}(t)\right]
$$

Como temos controle independente sobre ambos os campos, é possível ajustar as intensidades médias de modo que $\bar{I}_{\mathrm{LO}}>>\bar{I}$. Caso os ruídos sejam da mesma ordem,

$$
\Delta^{2} \hat{I}_{-} \approx K|g|^{2} \bar{I}_{\mathrm{LO}} \Delta^{2} \hat{q}_{\phi}(t)
$$

Uma situação interessante, e importante do ponto de vista experimental, ocorre quando o estado a ser medido possui média nula, como no caso dos estados gerados pelo OPO operando abaixo do limiar. Neste caso, $|\alpha|=0$ e a relação anterior se torna exata, assim como

$$
\bar{I}_{-}=0 \quad \text { e } \quad \hat{I}_{-}(t)=\delta \hat{I}_{-}(t)=\sqrt{K}|g| \bar{I}_{\mathrm{LO}} \delta \hat{q}_{\phi}(t)
$$

Consequentemente, a subtração da intensidade é proporcional à quadratura generalizada nas bandas laterais. Assim como ocorre para apenas um detetor, a intensidade da portadora amplifica o sinal presente nas bandas laterais. Entretanto, aqui é a portadora do oscilador local que faz esta amplificação. Além disso, a fase $\phi$ permite escolher qual quadratura será medida. Variando $\phi$ é possível reconstruir qualquer estado do modo temporal das bandas laterais por meio da tomografia homodina [25, 85, 24].

\subsection{Limite Quântico Padrão - Shot Noise}

Medições de qualquer sistema físico são sempre realizadas tendo uma referência fixa cuja precisão impõe um limite inferior na precisão com que é possível resolver as medidas realizadas. No nosso sistema, estamos interessados em medir as flutuações das quadraturas das bandas laterais. Para isso necessitamos de uma referência com a qual possamos compará-las, tendo em mente que antes de serem adquiridos no computador, os dados passam por circuitos eletrônicos que realizam diversas operações, como filtragens e amplificações. A referência que usamos é o ruído do estado de vácuo nas mesmas condições em que as quadraturas são medidas. Este ruído é chamado de Limite Quântico Padrão (SQL - do inglês Standard Quantum Limit) [86].

Como nossos detetores medem intensidade, eles são insensíveis ao estado de vácuo diretamente. Entretanto, usando a detecção homodina, podemos medir o mesmo 
de maneira indireta. Se o campo de interesse, $\hat{a}$, está no estado de vácuo, $|\alpha|=0$ e $\Delta^{2} \hat{q}_{-\phi}(t)=1$, portanto, a expressão 3.25 se torna

$$
\Delta^{2} \hat{I}_{-}=K|g|^{2} \bar{I}_{\mathrm{LO}}
$$

O ruído de subtração é proporcional à intensidade, o que caracteriza um processo

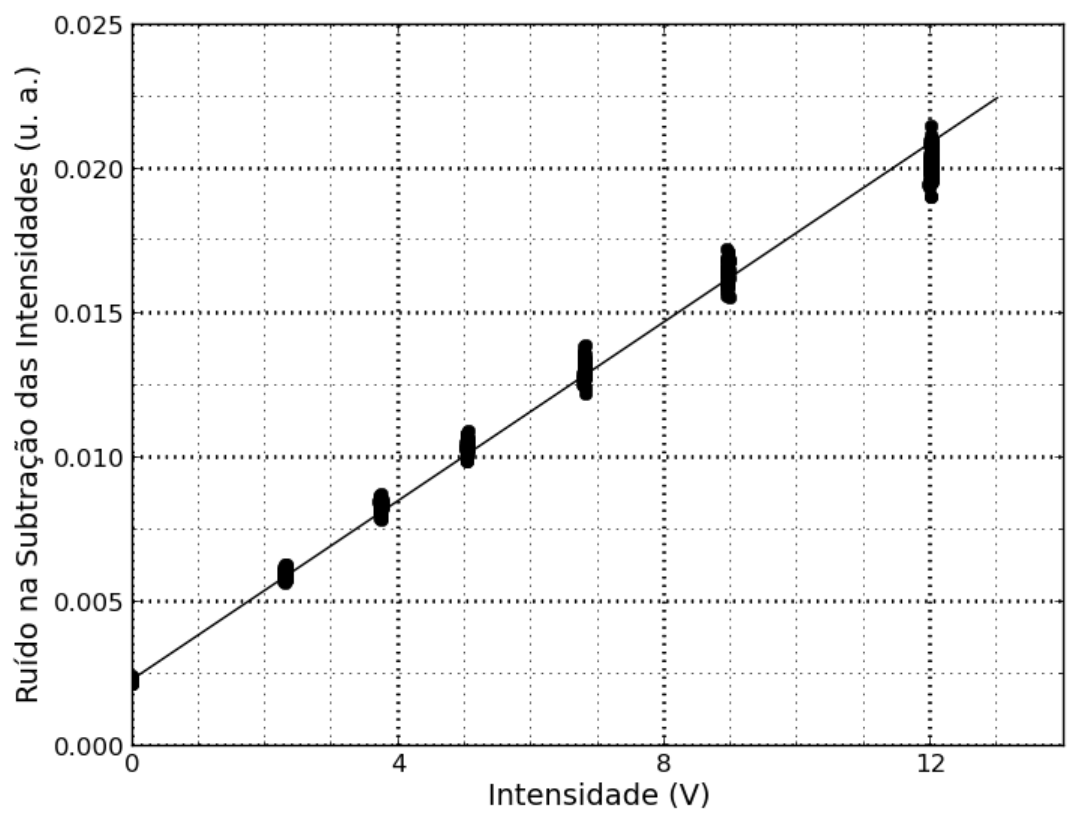

Figura 3.5: Ruído de subtração vs intensidade. Esta medida fornece a referência com a qual o ruído de quadratura é comparado e, como pode ser visto no gráfico, varia linearmente com a intensidade.

poissoniano. Essa propriedade ocorre devido à natureza granular da luz, composta por fótons, absorvida pelo detector e ao fato de a chegada de qualquer um deles ser descorrelacionada da do fóton anterior [87 . O ruído em 3.27) é chamado de shot noise e seu coeficiente angular em relação à intensidade fornece a constante de proporcionalidade que aparece na equação. Após calibrar o shot noise temos acesso às quadraturas de fato, pois

$$
\delta \hat{q}_{\phi}(t)=\frac{\delta \hat{I}_{-}(t)}{\sqrt{\Delta^{2} \hat{I}_{-} \bar{I}_{\mathrm{LO}}}}
$$

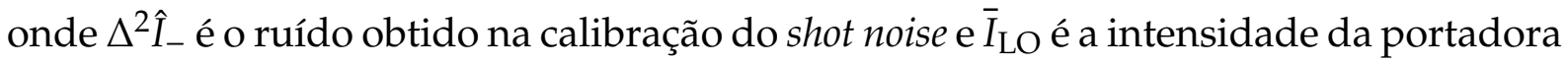
do oscilador local ótico.

\footnotetext{
${ }^{5}$ Um ruído com a mesma propriedade é observado ao se ouvir o barulho da chuva!
} 


\subsection{Medidas no Domínio da Frequência - Demodulação}

Como discutido até aqui, a detecção homodina é uma técnica bastante eficiente para medir as quadraturas do modo efetivo das bandas laterais resolvido no tempo. Porém, na prática, esta medida nem sempre é a mais adequada por causa da presença de ruído clássico em baixa frequência. O uso da detecção homodina balanceada diminui bastante este problema, mas não o elimina. Por este motivo, é comum realizar medidas no domínio da frequência selecionando somente uma das frequências de batimento, $\Omega$. Devido ao fato de cada frequência presente na fotocorrente ser resultado da mistura de dois modos das bandas laterais, a medida no domínio da frequência, por natureza, é bimodal. Entretanto, antes de tratar explicitamente das medidas no domínio da frequência, discutirei um importante teorema que impõe restrições nos segundos momentos calculados a partir de um processo estacionário.

\subsubsection{Teorema de Wiener-Khinchin e Fotocorrente Estacionária}

Um processo físico representado por uma variável aleatória $x(t)$ será chamado estacionário se possuir média sobre ensemble constante no tempo e se a função de correlação temporal for invariante por translação temporal $[x(t)\rangle=\langle x\rangle$ e $V_{c}\left(t, t^{\prime}\right)=\left\langle x(t) x\left(t^{\prime}\right)\right\rangle=$ $\langle x(0) x(\tau)\rangle=V_{c}(\tau)$, com $\tau=t-t^{\prime}$. O teorema de Wiener-Khinchin diz que a qualquer variável estacionária com primeiro e segundo momentos bem definidos - ou seja, $\langle x\rangle \mathrm{e}$ $V_{c}(\tau)$ não divergem - está associada uma densidade espectral que é uma transformada de Fourier da correlação temporal. Para mostrar o teorema, considere a transformada de Fourier de $x(t)$ [62],

$$
x(\Omega)=\int_{-\infty}^{+\infty} d t x(t) \mathrm{e}^{-i \Omega t} .
$$

A função de correlação desta quantidade em frequências distintas é

$$
\begin{gathered}
\left\langle x(\Omega) x\left(\Omega^{\prime}\right)\right\rangle=\int_{-\infty}^{+\infty} d t d t^{\prime}\left\langle x(t) x\left(t^{\prime}\right)\right\rangle \mathrm{e}^{-i \Omega t} \mathrm{e}^{-i \Omega^{\prime} t^{\prime}}=2 \pi S_{p}(\Omega) \delta\left(\Omega+\Omega^{\prime}\right) \\
\operatorname{com} \quad S_{p}(\Omega)=\langle x(\Omega) x(-\Omega)\rangle=\int_{-\infty}^{+\infty} d \tau V_{c}(\tau) \mathrm{e}^{-i \Omega \tau}
\end{gathered}
$$

\footnotetext{
${ }^{6}$ A palavra processo estacionário é usado na literatura com mais de um significado [88, 62]. Todos eles são similares, mas me atenho à definição dada neste texto, pois é a que mais diretamente se aplica ao trabalho apresentado.
} 
e $S_{p}(\Omega)$ é chamada de densidade espectral ou espectro de potêncid7] Se escrevemos a flutuação de $x(t)$ como $\delta x(t)=x(t)-\langle x\rangle$, a densidade espectral se torna

$$
S_{p}(\Omega)=2 \pi \delta(\Omega)\langle x\rangle^{2}+S(\Omega), \quad \text { onde } \quad S(\Omega)=\langle\delta x(\Omega) \delta x(-\Omega)\rangle=\int_{-\infty}^{+\infty} d \tau V(\tau) \mathrm{e}^{-i \Omega \tau}
$$

e $V(\tau)=\langle\delta x(0) \delta x(\tau)\rangle$. A quantidade $S(\Omega)$ é chamada de espectro de ruído e somente difere do espectro de potência na frequência zero. Neste ponto, o delta de Dirac forma uma singularidade removível e em todos os outros pontos $S_{p}(\Omega)$ e $S(\Omega)$ carregam a mesma informação. Note que como a densidade espectral está multiplicada por $\delta\left(\Omega+\Omega^{\prime}\right)$, a quantidade $\langle\delta x(\Omega) \delta x(\Omega)\rangle \approx 0$ para qualquer frequência $\Omega$.

No contexto quântico, um processo físico $x(t)$ não é mais representado por uma variável aleatória, mas por um operador hermitiano. Para adaptar o teorema a este contexto, as médias devem ser calculadas da forma $\langle\hat{x}(t)\rangle=\operatorname{Tr}[\hat{x}(t) \hat{\rho}]$, onde $\hat{\rho}$ é o operador densidade no formalismo de Heisenberg. O teorema de Wiener-Khinchin ainda é válido se ambos o primeiro e segundo momentos estão bem definidos e, como $\hat{x}(0)$ e $\hat{x}(\tau)$ podem não comutar, $V(\tau)$ é calculada na ordem simétrica, pois é a única ordenação na qual a mesma é real, como será explicitado mais à frente.

\subsubsection{Filtro passa-faixa}

Inicialmente discutirei a medida direta no domínio da frequência utilizando um filtro passa-faixa para filtrar a componente da fotocorrente que oscila apenas na frequência de interesse. Entretanto, por conveniência experimental, utilizamos um sistema de demodulação que permite converter a informação na frequência de interesse para o sinal DC (do inglês Direct Current), que representa a informação de baixa frequência. O sinal resultante de ambos procedimentos são similares e por este motivo, discutirei o filtro passa-faixa primeiro por ser mais simples. Inicialmente, considere que passamos a fotocorrente por um filtro passa-banda eletrônico, com frequência central de $\Omega_{\mathrm{an}}$, chamada frequência de análise, largura de banda de $\Delta \Omega_{\mathrm{pb}}$ e perfil lorentziano,

$$
G\left(\Omega_{\mathrm{an}}-\Omega\right)=\frac{1}{1+\frac{4\left(\Omega_{\mathrm{an}}-\Omega\right)^{2}}{\Delta \Omega_{\mathrm{pb}}^{2}}}
$$

\footnotetext{
${ }^{7}$ Note que $S_{p}(\Omega)$ possui dimensão de $[x(t)]^{2}\left[\right.$ tempo] enquanto a dimensão de $\left\langle x(\Omega) x\left(\Omega^{\prime}\right)\right\rangle$ é de $[x(t)]^{2}[\text { tempo }]^{2}$.
} 
Ao passar pelo filtro, o operador temporal em (3.14), quando consideramos a expansão de Fourier em termos somente das componentes de frequência positiva, se torna

$$
\begin{aligned}
\delta \hat{q}_{\phi}(t) & =\int_{0}^{+\infty} d \Omega G\left(\Omega-\Omega_{\mathrm{an}}\right)\left[\delta \hat{q}_{\phi}(\Omega) \mathrm{e}^{-i \Omega t}+\left[\delta \hat{q}_{\phi}(\Omega)\right]^{\dagger} \mathrm{e}^{i \Omega t}\right] \\
& \approx\left[\delta \hat{q}_{\phi}\left(\Omega_{\mathrm{an}}\right) \mathrm{e}^{-i \Omega_{\mathrm{an}} t}+\delta \hat{q}_{\phi}\left(-\Omega_{\mathrm{an}}\right) \mathrm{e}^{i \Omega_{\mathrm{an}} t}\right],
\end{aligned}
$$

pois o perfil do filtro se aproxima de uma delta de Dirac para $\Delta \Omega_{\mathrm{pb}}$ muito pequeno. Em um primeiro momento, vamos olhar apenas para o ruído de $\delta \hat{q}_{\phi}(t)$ e conectar com o Teorema de Wiener-Khinchin. Partindo da equação (3.35), vemos que

$$
\begin{aligned}
\left\langle\left[\delta \hat{q}_{\phi}(t)\right]^{2}\right\rangle= & \left\langle\delta \hat{q}_{\phi}\left(\Omega_{\mathrm{an}}\right) \delta \hat{q}_{\phi}\left(-\Omega_{\mathrm{an}}\right)\right\rangle+\left\langle\delta \hat{q}_{\phi}\left(-\Omega_{\mathrm{an}}\right) \delta \hat{q}_{\phi}\left(\Omega_{\mathrm{an}}\right)\right\rangle+ \\
& \left\langle\delta \hat{q}_{\phi}\left(\Omega_{\mathrm{an}}\right) \delta \hat{q}_{\phi}\left(\Omega_{\mathrm{an}}\right)\right\rangle \mathrm{e}^{-2 i \Omega_{\mathrm{an}} t}+\left\langle\delta \hat{q}_{\phi}\left(-\Omega_{\mathrm{an}}\right) \delta \hat{q}_{\phi}\left(-\Omega_{\mathrm{an}}\right)\right\rangle \mathrm{e}^{2 i \Omega_{\mathrm{an}} t} \\
= & 2 \operatorname{Re}\left[\left\langle\delta \hat{q}_{\phi}\left(\Omega_{\mathrm{an}}\right) \delta \hat{q}_{\phi}\left(-\Omega_{\mathrm{an}}\right)\right\rangle\right]+2 \operatorname{Re}\left[\left\langle\delta \hat{q}_{\phi}\left(\Omega_{\mathrm{an}}\right) \delta \hat{q}_{\phi}\left(\Omega_{\mathrm{an}}\right)\right\rangle \mathrm{e}^{-2 i \Omega_{\mathrm{an}} t}\right](3.3
\end{aligned}
$$

Somente o segundo termo de (3.37) depende do tempo. Ele também depende de $\left\langle\delta \hat{q}_{\phi}\left(\Omega_{\mathrm{an}}\right) \delta \hat{q}_{\phi}\left(\Omega_{\mathrm{an}}\right)\right\rangle$ que, segundo o Teorema de Wiener-Khinchin, se anula em um processo estacionário. Se a fotocorrente for estacionária, o ruído do operador de quadratura é independente do tempo,

$$
\begin{aligned}
\left\langle\left[\delta \hat{q}_{\phi}(t)\right]^{2}\right\rangle & =2 \operatorname{Re}\left[\left\langle\delta \hat{q}_{\phi}\left(\Omega_{\mathrm{an}}\right) \delta \hat{q}_{\phi}\left(-\Omega_{\mathrm{an}}\right)\right\rangle\right] \\
& =\cos ^{2} \phi \frac{\left\langle\hat{q}_{+}^{2}\right\rangle+\left\langle\hat{p}_{-}^{2}\right\rangle}{2}+\operatorname{sen}^{2} \phi \frac{\left\langle\hat{p}_{+}^{2}\right\rangle+\left\langle\hat{q}_{-}^{2}\right\rangle}{2}+\operatorname{sen}(2 \phi) \frac{\left\langle\hat{q}_{+} \hat{p}_{+}\right\rangle_{S}-\left\langle\hat{q}_{-} \hat{p}_{-}\right\rangle_{S_{3}}}{2} .
\end{aligned}
$$

onde $\left\langle\hat{q}_{ \pm} \hat{p}_{ \pm}\right\rangle_{S}$ significa que os operadores são calculados em ordem simétrica. Conse-
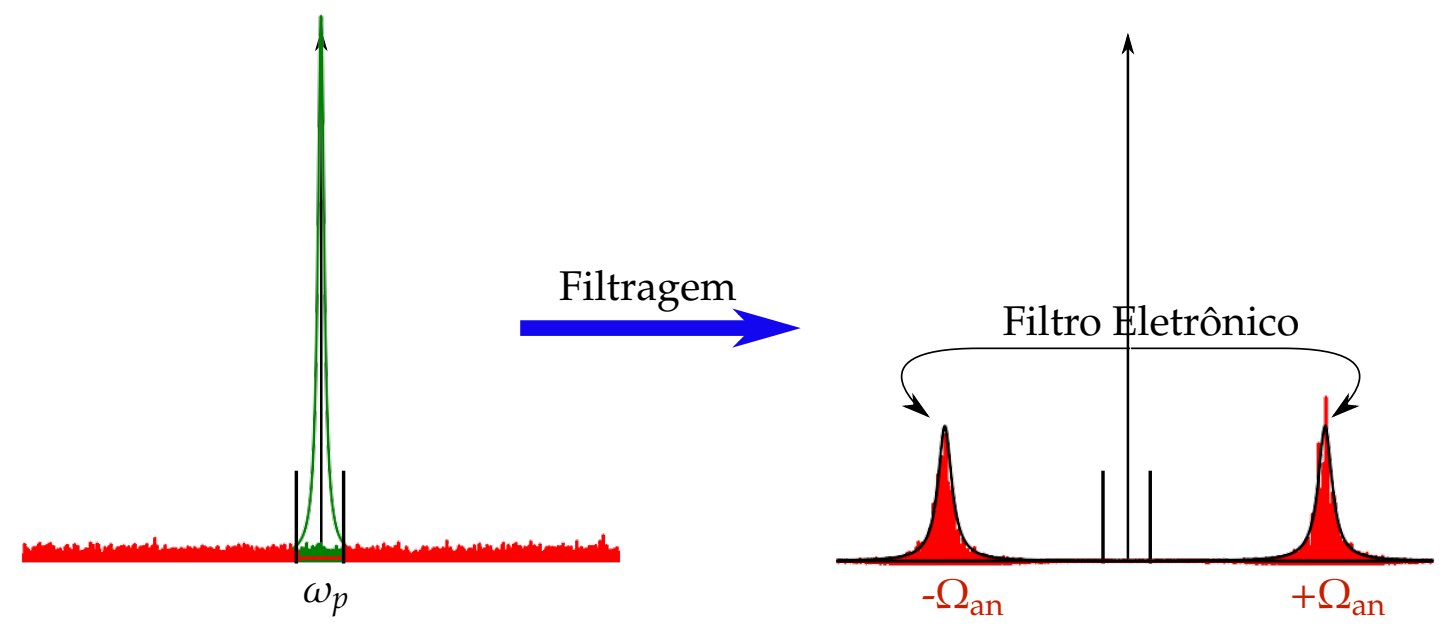

Figura 3.6: O filtro passa-faixa separa a informação somente das componentes de frequência em torno da frequência de análise.

quentemente, o espectro de ruído referente à detecção homodina espectral é, intrinse- 
camente, uma mistura estatística de ruídos e correlações de operadores nos subespaços de soma e subtração de bandas laterais. Uma das consequências diretas desta mistura é que o squeezing espectral, por exemplo na quadratura fase 8 , é equivalente ao emaranhamento entre as bandas laterais, pois

$$
\left\langle\delta \hat{q}_{\pi / 2}\left(\Omega_{\mathrm{an}}\right) \delta \hat{q}_{\pi / 2}\left(-\Omega_{\mathrm{an}}\right\rangle<1 \quad \Longrightarrow \quad\left\langle\hat{p}_{+}^{2}\right\rangle+\left\langle\hat{q}_{-}^{2}\right\rangle<2\right.
$$

Esta última desigualdade é a violação do critério $D G C Z$ de emaranhamento [89]. A estacionariedade impõe vínculos sobre os segundos momentos de $\hat{q}_{ \pm}$, pois como

$$
\begin{array}{r}
\operatorname{Re}\left[\left\langle\delta \hat{q}_{\phi}\left(\Omega_{\mathrm{an}}\right) \delta \hat{q}_{\phi}\left(\Omega_{\mathrm{an}}\right)\right\rangle\right]=\cos ^{2} \phi \frac{\left\langle\hat{q}_{+}^{2}\right\rangle-\left\langle\hat{p}_{-}^{2}\right\rangle}{2}+\operatorname{sen}^{2} \phi \frac{\left\langle p_{+}^{2}\right\rangle-\left\langle\hat{q}_{-}^{2}\right\rangle}{2} \\
+\operatorname{sen}(2 \phi) \frac{\left\langle\hat{q}_{+} \hat{p}_{+}\right\rangle_{S}+\left\langle\hat{q}_{-} \hat{p}_{-}\right\rangle_{S}}{2}=0,
\end{array}
$$

os subespaços de soma e subtração de bandas laterais possuem a mesma estatística, $\left\langle\hat{q}_{+}^{2}\right\rangle=\left\langle\hat{p}_{-}^{2}\right\rangle,\left\langle\hat{p}_{+}^{2}\right\rangle=\left\langle\hat{q}_{-}^{2}\right\rangle,\left\langle\hat{q}_{+} \hat{p}_{+}\right\rangle_{S}=-\left\langle\hat{q}_{-} \hat{p}_{-}\right\rangle_{S}$. Nestas condições, as matrizes de covariâncias de cada um dos modos $\hat{q}_{+}$e $\hat{q}_{-}$são iguais, a menos de uma fase de $\pi / 2$ em $\hat{q}_{-}$, e podem ser medidas diretamente pela detecção homodina espectral. Consequentemente, se assumimos que os estados reduzidos de $\hat{q}_{+}$e $\hat{q}_{-}$são gaussianos, estes podem ser medidos completamente e são iguais. Portanto, para estados gaussianos e estacionários, a detecção homodina espectral fornece, intrinsecamente, uma descrição efetiva de um modo por campo - podendo este ser $\hat{q}_{+}$ou $\hat{q}_{-}$- para a medida bimodal realizada na fotodetecção.

Caso não haja correlações entre os subespaços de soma e subtração, a informação presente nas matrizes de covariâncias dos subespaços de soma e subtração já corresponde à informação completa disponível. Entretanto, caso estas correlações sejam não-nulas, a detecção homodina espectral é insensível às mesmas. Esta limitação da detecção homodina espectral será discutida em mais detalhes mais adiante. É importante também analisar o sistema sem a necessidade de assumir a condição de fotocorrente estacionária, pois isto nos permite derivar quantidades que atestam a validade desta hipótese. Para isso, o operador espectral $\delta \hat{q}_{\phi}\left(\Omega_{\mathrm{an}}\right)$ é de pouca utilidade e teremos que trabalhar diretamente com os operadores de soma e subtração das bandas laterais. Isto será feito na próxima seção na qual discutirei explicitamente a forma como medimos as componentes espectrais da fotocorrente.

\footnotetext{
${ }^{8}$ Este exemplo é interessante, pois é o estado previsto para ser produzido em um OPO degenerado operando abaixo do limiar [54].
} 


\subsubsection{Demodulação}

Isolar uma componente de frequência do ruído usando um filtro passa-faixa pode apresentar duas dificuldades técnicas. A primeira delas é que, para ter sensitividade em um sinal que oscila na frequência de análise, precisamos medir em escalas de tempo, pelo menos, da ordem de $\Omega_{a n}^{-1}$. Se a frequência de análise for muito grande, este é um fator limitante para a medida. A segunda dificuldade surge quando é necessário medir em outra frequência de análise. Para isso teríamos que modificar o filtro passa-faixa, o que implica em modificar componentes dentro do circuitd?

Por conveniência, usamos um circuito de demodulação em vez de um filtro passafaixa. Ele converte a informação contida na frequência de análise para a frequência nula (sinal DC). A aquisição pode então ser feita em um osciloscópio ou um em um computador por meio de um conversor analógico/digital. O demodulador é um circuito não-linear, formado por um misturador eletrônico (mixer), um oscilador de frequência controlada (oscilador local eletrônico) e um filtro passa-baixa. O sinal de entrada $\delta \hat{i}(t)$ como em (3.11) é misturado com o oscilador local eletrônico na frequência $\Omega_{\mathrm{an}}$

$$
i_{L O}^{c}(t)=\frac{1}{2}\left[I_{0} e^{-i \Omega_{\mathrm{an}} t}+\text { c.c. }\right] .
$$

chamado de componente cosseno. Os dois sinais são multiplicados no mixer resultando em

$$
\begin{aligned}
\delta \hat{i}_{\text {mix }}(t) & =\delta \hat{i}(t) i_{L O}^{c}(t)=\frac{1}{2} \int_{-\infty}^{+\infty} d \Omega^{\prime}\left[I_{0} e^{-i\left(\Omega_{\mathrm{an}}+\Omega^{\prime}\right) t}+I_{0}^{*} e^{i\left(\Omega_{\mathrm{an}}-\Omega^{\prime}\right) t}\right] \delta \hat{i}\left(\Omega^{\prime}\right) \\
\delta \hat{i}_{\text {mix }}(\Omega) & =\frac{1}{2 \pi} \int_{-\infty}^{+\infty} d t \delta \hat{i}_{\text {mix }}(t) e^{i \Omega t}=\frac{1}{2}\left[I_{0} \delta \hat{i}\left(\Omega-\Omega_{\mathrm{an}}\right)+I_{0}^{*} \delta \hat{i}\left(\Omega+\Omega_{\mathrm{an}}\right)\right],
\end{aligned}
$$

$\operatorname{com} \delta \hat{i}(\Omega)=K\left|\alpha_{p} \| g\right| \delta \hat{q}_{\phi}(\Omega)$. O filtro passa-baixa vai selecionar apenas frequências próximas de zero. No caso ideal, o filtro é descrito por uma delta de Dirac centrada na origem. Após o filtro, cada componente espectral será transformada como

$$
\delta \hat{i}_{\text {out }}^{c}(\Omega)=\delta_{\text {Dirac }}(\Omega) \delta \hat{i}_{\text {mix }}(\Omega) .
$$

Portanto, a fotocorrente de entrada $\delta \hat{i}(t)$ é convertida para

$$
\delta \hat{i}_{\text {out }}^{c}(t)=\delta \hat{i}_{\text {out }}^{c}=\int_{-\infty}^{+\infty} d \Omega \delta \hat{i}_{\text {out }}(\Omega) e^{-i \Omega t}=\frac{K\left|\alpha_{p} \| g\right|}{2}\left[I_{0} \delta \hat{q}_{\phi}\left(-\Omega_{\mathrm{an}}\right)+I_{0}^{*} \delta \hat{q}_{\phi}\left(\Omega_{\mathrm{an}}\right)\right] .
$$

\footnotetext{
${ }^{9}$ Filtro passa-faixa geralmente são feitos com circuitos LC e é possível variar a frequência central do filtro usando um indutor variável. Porém este ajustes são feitos diretamente no componente eletrônico.
} 


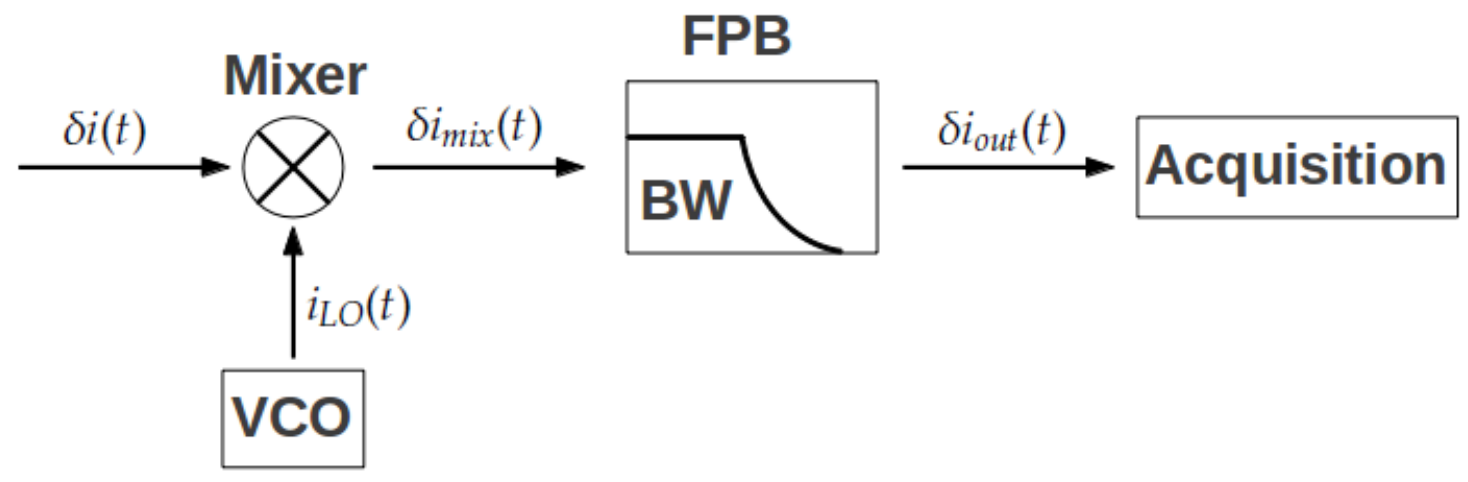

Figura 3.7: Diagrama esquemático mostrando o processo de demodulação. O sinal gerado pelo oscilador local eletrônico é misturado ao sinal de entrada. O resultado é enviado para um filtro passa-baixa e depois adquirido em um osciloscópio ou conversor analógico-digital.VCO representa o oscilador local (voltage controlled oscillator), $\mathbf{F P B}$, o filtro passa-baixa e $\mathbf{B W}$ representa a frequência de corte do filto (bandwidth).

Diferente do que ocorre em (3.35), o sinal medido não oscila. A flutuação de intensidade de saída é proporcional às flutuações do campo de entrada que oscilam na frequência do oscilador local eletrônico. Como o filtro passa-baixa é considerado ideal, as flutuações de saída não dependem do tempo. De fato, os valores médios calculados devem ser entendidos como médias sobre ensembles em que cada ponto adquirido corresponde a uma realização do experimento. Em um experimento real, o filtro passa-baixa terá uma largura, ajustada no circuito eletrônico, que irá fazer com que $\delta i_{\text {out }}^{c}$ varie em uma escala de tempo da ordem do inverso desta largura. Em função dos operadores no espaço de soma e subtração de bandas laterais, (3.45) se torna

$$
\begin{gathered}
\delta \hat{i}_{\text {out }}^{c} \sim \hat{q}_{+}(\theta) \cos \phi+\hat{p}_{+}(\theta) \operatorname{sen} \phi \\
\hat{q}_{+}(\theta)=\hat{q}_{+} \cos \theta-\hat{p}_{-} \operatorname{sen} \theta \text { e } \hat{p}_{+}(\theta)=\hat{p}_{+} \cos \theta+\hat{q}_{-} \operatorname{sen} \theta,
\end{gathered}
$$

onde $\theta$ é a fase de $I_{0}^{*}$ em relação a $\delta \hat{i}(\Omega)$, e o ruído de $\delta \hat{i}_{\text {out }}^{c}$ se torna

$$
\left\langle\delta \hat{i}_{\text {out }}^{c} \delta \hat{i}_{\text {out }}^{c}\right\rangle \sim \operatorname{Re}\left[\left\langle\delta \hat{q}_{\phi}\left(\Omega_{\mathrm{an}}\right) \delta \hat{q}_{\phi}\left(-\Omega_{\mathrm{an}}\right)\right\rangle\right]+\operatorname{Re}\left[\mathrm{e}^{2 i \theta}\left\langle\delta \hat{q}_{\phi}\left(\Omega_{\mathrm{an}}\right) \delta \hat{q}_{\phi}\left(\Omega_{\mathrm{an}}\right)\right\rangle\right],
$$

Note que a expressão acima é similar a (3.37), encontrada com o filtro passa-faixa, porém a fase que multiplica $\left\langle\delta \hat{q}_{\phi}\left(\Omega_{\mathrm{an}}\right) \delta \hat{q}_{\phi}\left(\Omega_{\mathrm{an}}\right)\right\rangle$ é ajustável e não possui qualquer relação com a frequência de análise. As componentes de frequências positiva e negativa, mostradas em (3.35) e (3.46) são uma conveniência matemática quando analisamos somente a fotocorrente. Porém, como mostrado em (3.12) ambas as componentes eletrônicas correspondem a duas componentes de frequência positiva óticas. O resultado da 
demodulação é a medida de um modo efetivo, formado pelos modos das bandas laterais superior e inferior, associado ao seguinte operador de criação,

$$
\hat{a}_{+}(\theta)=\hat{a}_{+} \cos (\theta)+i \hat{a}_{-} \operatorname{sen}(\theta)
$$

com $\hat{a}_{ \pm}$definidos em (3.15). Este operador pode ser entendido graficamente como um interferômetro Mach-Zehnder como mostrado na figura 3.8. O resultado da medida associada à detecção homodina espectral é equivalente a uma das saídas do último divisor de feixes.

\subsubsection{Dupla Demodulação}

A outra saída do Mach-Zehnder também tem sentido físico e está associada à demodulação com um oscilador local em quadratura com $\hat{i}_{L O}^{c}(t)$. Ambas as saídas podem ser obtidas por meio de uma dupla demodulação Este outro oscilador local eletrônico, em quadratura com $\delta \hat{i}_{\text {out }}^{c}$, é dado por

$$
\delta \hat{i}_{\text {out }}^{s}=\frac{1}{2 i}\left[I_{0} \delta \hat{i}\left(-\Omega_{\mathrm{an}}\right)-I_{0}^{*} \delta \hat{i}\left(\Omega_{\mathrm{an}}\right)\right]
$$

e é chamado de componente seno. Assim com em (3.45), o sinal de saída aqui será

$$
\delta \hat{i}_{\text {out }}^{\mathrm{s}}=\frac{K\left|\alpha_{p} \| g\right|}{2 i}\left[I_{0} \delta \hat{q}_{\phi}\left(-\Omega_{\mathrm{an}}\right)-I_{0}^{*} \delta \hat{q}_{\phi}\left(\Omega_{\mathrm{an}}\right)\right] .
$$

A demodulação com dois osciladores locais fornece dois operadores de medidas, um resulta da demodulação usando a componente cosseno do oscilador local eletrônico, como mostrado em 3.46, e outro usando a componente seno,

$$
\begin{gathered}
\delta \hat{i}_{\text {out }}^{s} \sim \hat{p}_{-}(\theta) \cos \phi-\hat{q}_{-}(\theta) \operatorname{sen} \phi \\
\hat{p}_{-}(\theta)=\hat{p}_{-} \cos \theta+\hat{q}_{+} \operatorname{sen} \theta \text { e } \hat{q}_{-}(\theta)=\hat{q}_{-} \cos \theta-\hat{p}_{+} \operatorname{sen} \theta
\end{gathered}
$$

Os operadores $\hat{q}_{ \pm}(\theta)$ e $\hat{p}_{ \pm}(\theta)$ formam variáveis conjugadas, pois

$$
\left.\left[\hat{q}_{ \pm}(\theta), \hat{p}_{ \pm}(\theta]\right)\right]=2 i \quad \text { e } \quad\left[\hat{q}_{+}(\theta), \hat{q}_{-}(\theta)\right]=\left[\hat{p}_{+}(\theta), \hat{p}_{-}(\theta)\right]=\left[\hat{q}_{+}(\theta), \hat{p}_{-}(\theta)\right]=0
$$

O operador (3.52) está associado ao seguinte operador de aniquilação,

$$
\hat{a}_{-}(\theta)=\hat{a}_{-} \cos (\theta)-i \hat{a}_{+} \operatorname{sen}(\theta) \text {. }
$$

que é o operador resultante da outra saída do Mach-Zehnder mostrado na figura 3.8 . 


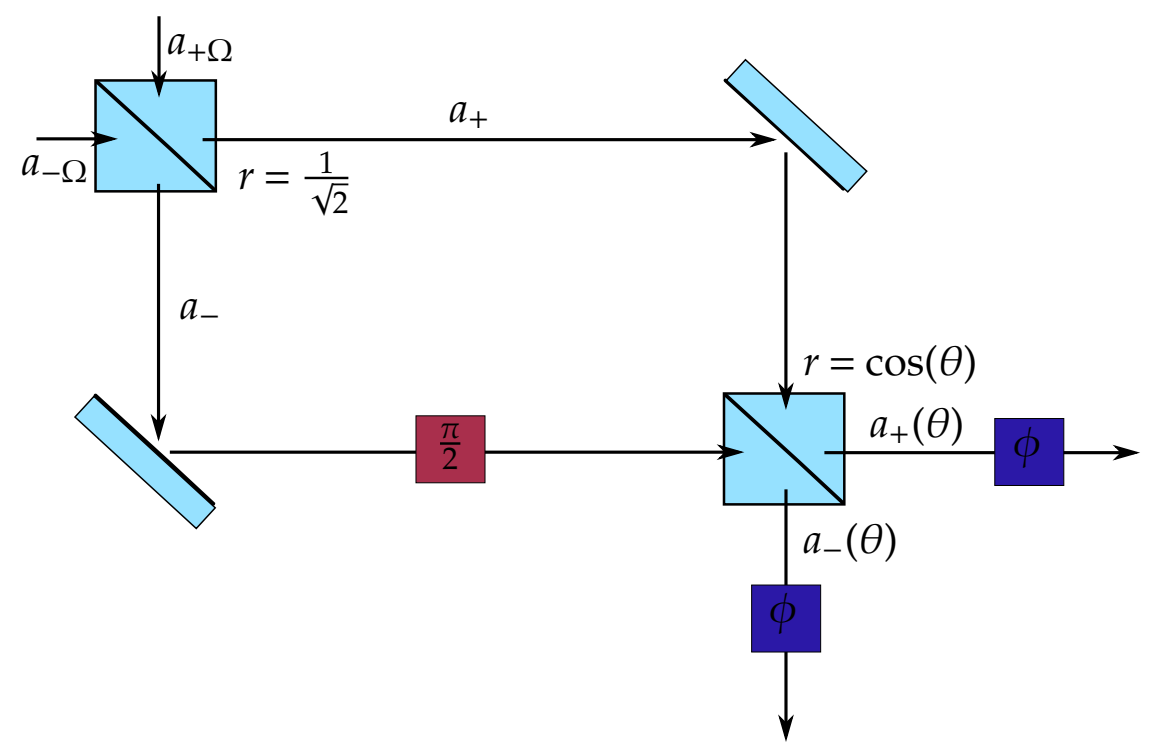

Figura 3.8: Representação pictórica da fotodetecção no dominio da frequêcia como um inteferômetro Mach-Zehnder com fase relativa de $\pi$ fixada entre os braços. O parâmetro $r$ é a amplitude de reflexão do divisor de feixes, de modo que os coeficientes de reflexão e transmissão sejam dados por $R=r^{2}$ e $T=1-r^{2}$.

Se escolhemos $\theta=0, \pi / 2, \pi$ ou $3 \pi / 2$, é possível medir $\hat{a}_{+}$e $\hat{a}_{-}$, porém nunca teremos acesso a apenas uma das bandas laterais, pois a fase de $\pi$ introduzida pela própria medida impede que haja alguma superposição de $\hat{a}_{+}$e $\hat{a}_{-}$no segundo divisor de feixes que resulte em $\hat{a}_{+\Omega}$ ou $\hat{a}_{-\Omega}$. Usando a detecção homodina, é possível controlar a fase $\phi$ e reconstruir o estado do modo temporal após o filtro. Neste ponto fica clara a limitação da detecção homodina. É possível reconstruir completamente por meio da tomografia homodina [24] o estado individual dos modos $\hat{q}_{ \pm}(\theta)$, que representam cada uma das saídas do Mach-Zehnder. Uma reconstrução completa seria possível se fases independentes fossem introduzidas em cada saída. Porém, como a tomografia é realizada com a mesma fase $\phi$ em ambas, não é possível observar todas as correlações entre os modos $\hat{q}_{+}$e $\hat{q}_{-}$. O fato de, com a liberdade de controle de $\theta$, ser possível modificar os pesos de $\hat{q}_{+}$e $\hat{p}_{-}$em $\hat{q}_{+}(\theta)$ e de $\hat{q}_{-}$e $\hat{p}_{+}$em $\hat{q}_{-}(\theta)$, nos permite medir algumas das correlações entre os subespaços de soma e subtração de bandas laterais, mas não todas. Esta limitação pode ser vista mesmo em estados gaussianos, os quais são completamente caracterizados pelos segundos momentos. Para tal, é mais conveniente voltar a trabalhar com o operador $\delta \hat{q}_{\phi}\left(\Omega_{\mathrm{an}}\right)$. O ruído de $\delta \hat{i}_{\text {out }}^{\mathrm{s}}$ é dado por

$$
\left\langle\delta \hat{i}_{\text {out }}^{s} \delta \hat{i}_{\text {out }}^{s}\right\rangle \sim \operatorname{Re}\left[\left\langle\delta \hat{q}_{\phi}\left(\Omega_{\mathrm{an}}\right) \delta \hat{q}_{\phi}\left(-\Omega_{\mathrm{an}}\right)\right\rangle\right]-\operatorname{Re}\left[e^{2 i \theta}\left\langle\delta \hat{q}_{\phi}\left(\Omega_{\mathrm{an}}\right) \delta \hat{q}_{\phi}\left(\Omega_{\mathrm{an}}\right)\right\rangle\right] .
$$

Em conjunto com 3.48$)$, esta expressão pode ser usada para medir $\left\langle\delta \hat{q}_{\phi}\left(\Omega_{\mathrm{an}}\right) \delta \hat{q}_{\phi}\left(\Omega_{\mathrm{an}}\right)\right\rangle$, 


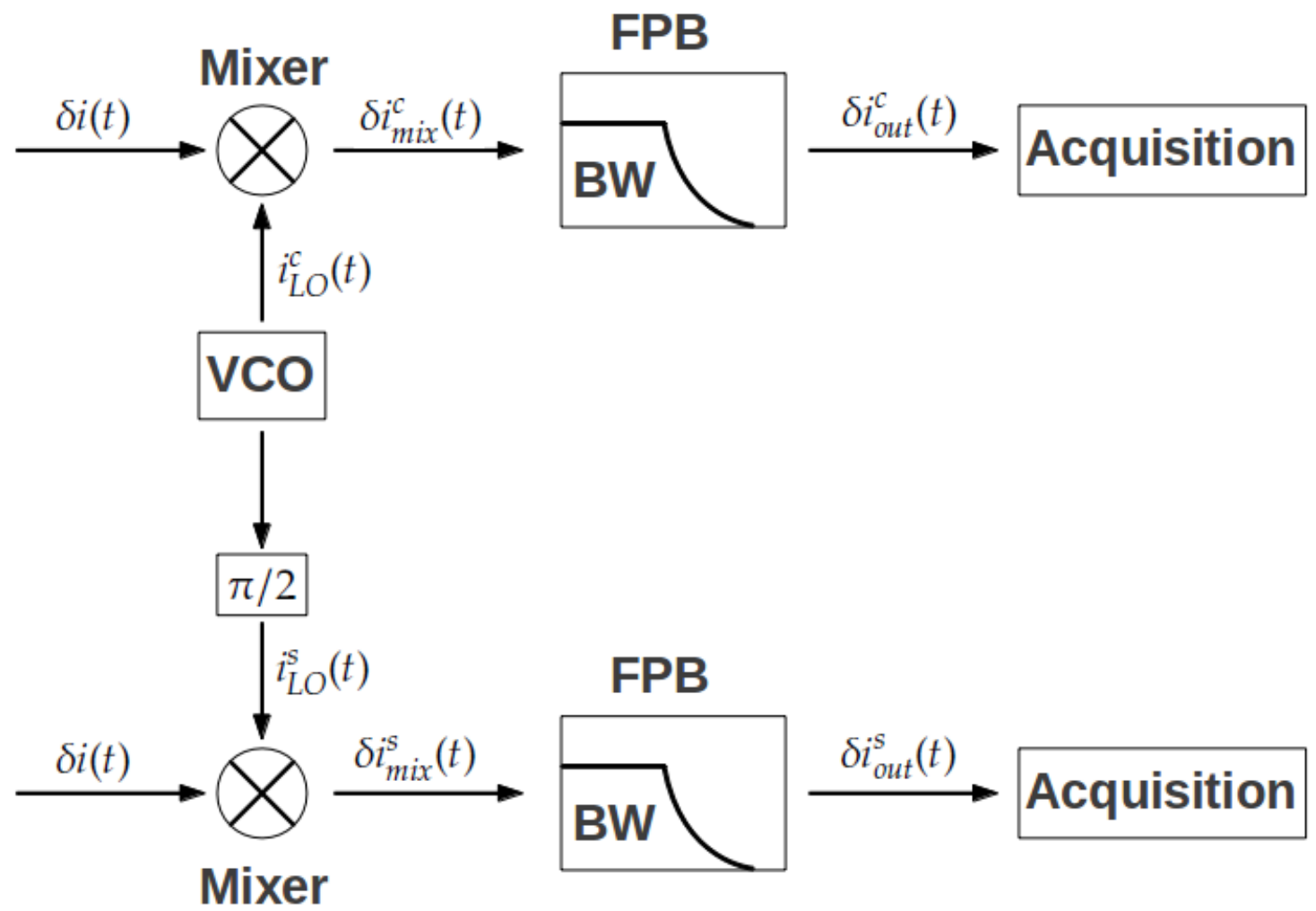

Figura 3.9: Demodulação feita com dois osciladores locais em quadratura. Por meio deste esquema podemos ter acesso à parte imaginária do espectro de ruído da fotocorrente.

pois este termo é proporcional à diferença entre $\left\langle\delta \hat{i}_{\text {out }}^{c} \delta \hat{i}_{\text {out }}^{c}\right\rangle$ e $\left\langle\delta \hat{i}_{\text {out }}^{s} \delta \hat{i}_{\text {out }}^{s}\right\rangle$. Consequentemente, se a fotocorrente é descrita por um processo estacionário, não há diferença entre os dois ruídos. Como podemos dividir a fotocorrente em duas partes iguais e demodular cada uma usando as componentes seno e cosseno como referência, podemos calcular também as correlações entre os sinais de saída,

$$
\left\langle\delta \hat{i}_{\text {out }}^{c} \delta \hat{i}_{\text {out }}^{\text {s }}\right\rangle=\left\langle\delta \hat{i}_{\text {out }}^{s} \delta \hat{i}_{\text {out }}^{c}\right\rangle \sim \operatorname{Im}\left[e^{2 i \theta}\left\langle\delta \hat{q}_{\phi}\left(\Omega_{\mathrm{an}}\right) \delta \hat{q}_{\phi}\left(\Omega_{\mathrm{an}}\right)\left(\Omega_{\mathrm{an}}\right)\right\rangle\right],
$$

pois $\left\langle\delta \hat{q}_{\phi}\left(\Omega_{\mathrm{an}}\right) \delta \hat{q}_{\phi}\left(-\Omega_{\mathrm{an}}\right)\right\rangle$ é real. Na correlação, o termo que depende de $\theta$ também é o que se anula em um processo estacionário. Em termos dos operadores nos subespaços de soma e subtração, o ruídos (3.48) e (3.56) e a correlação (3.57) se tornam

$$
\begin{aligned}
\left\langle\delta \hat{i}_{\text {out }}^{c} \delta \hat{i}_{\text {out }}^{c}\right\rangle \sim & \left\langle\hat{q}_{+}(\theta)^{2}\right\rangle \cos ^{2} \phi+\left\langle\hat{p}_{+}(\theta)^{2}\right\rangle \operatorname{sen}^{2} \phi+\left\langle\hat{q}_{+}(\theta) \hat{p}_{+}(\theta)\right\rangle_{S} \operatorname{sen}(2 \phi), \\
\left\langle\delta \hat{i}_{\text {out }}^{s} \delta \hat{i}_{\text {out }}^{s}\right\rangle \sim & \left\langle\hat{p}_{-}(\theta)^{2}\right\rangle \cos ^{2} \phi+\left\langle\hat{q}_{-}(\theta)^{2}\right\rangle \operatorname{sen}^{2} \phi-\left\langle\hat{q}_{-}(\theta) \hat{p}_{-}(\theta)\right\rangle_{S} \operatorname{sen}(2 \phi) \\
\left\langle\delta \hat{i}_{\text {out }}^{c} \delta \hat{i}_{\text {out }}^{s}\right\rangle \sim & \left\langle\hat{q}_{+}(\theta) \hat{p}_{-}(\theta)\right\rangle \cos ^{2} \phi-\left\langle\hat{p}_{+}(\theta) \hat{q}_{-}(\theta)\right\rangle \operatorname{sen}^{2} \phi+ \\
& \frac{\left\langle\hat{p}_{+}(\theta) \hat{p}_{-}(\theta)\right\rangle-\left\langle\hat{q}_{+}(\theta) \hat{q}_{-}(\theta)\right\rangle}{2} \operatorname{sen}(2 \phi),
\end{aligned}
$$


Ao variar a fase $\phi$ do oscilador local ótico é possível medir as matrizes de covariâncias dos subespaços de soma e subtração através de (3.58) e (3.59). Algumas das correlações podem ser medidas através de (3.60), porém, independentemente da fase $\theta$ do oscilador local eletrônico, não é possível medir

$$
\delta_{\mathrm{u}}(\theta)=\left\langle\hat{p}_{+}(\theta) \hat{p}_{-}(\theta)\right\rangle+\left\langle\hat{q}_{+}(\theta) \hat{q}_{-}(\theta)\right\rangle=\operatorname{Im}\left[\delta \hat{q}_{0}\left(\Omega_{\mathrm{an}}\right) \delta \hat{q}_{\pi / 2}\left(-\Omega_{\mathrm{an}}\right)\right] .
$$

como pode ser checado diretamente usando (3.14). Esta quantidade não se anula quando a fotocorrente é estacionária e está ligada ao desbalanceamento dos ruídos nas bandas laterais superior e inferior, pois

$\delta_{\mathrm{u}}(\theta)=\Delta^{2} \hat{q}_{+\Omega}(\theta)+\Delta^{2} \hat{p}_{+\Omega}(\theta)-\Delta^{2} \hat{q}_{-\Omega}(\theta)-\Delta^{2} \hat{p}_{-\Omega}(\theta) \quad$ onde $\quad \hat{a}_{ \pm \Omega}(\theta)=\frac{1}{\sqrt{2}}\left[\hat{a}_{+}(\theta) \pm \hat{a}_{-}(\theta)\right]$ A detecção homodina espectral é insensível a $\delta_{\mathrm{u}}(\theta)$ porque trata as bandas laterais da mesma maneira. Para medir $\delta_{\mathrm{u}}(\theta)$ é necessário introduzir desbalanceamento entre as bandas laterais, o que pode ser feito com a detecção com cavidades que será tratada mais à frente. Na próxima secção discutirei o papel da fase do oscilador local eletrônico, especialmente quando não temos controle da mesma.

\subsubsection{Difusão de Fase}

A fase dos osciladores locais eletrônicos é tipicamente bem estabilizada no tempq Porém, a fase da fotocorrente $\delta i(\Omega)$ está diretamente ligada à fase da luz que a gerou que, no caso de um laser, varia aleatoriamente no tempq ${ }^{11}$. Portanto, se as médias são calculadas em uma escala de tempo de mesma ordem que a variação de $\theta$, a componente cosseno no começo da medida pode se tornar a componente seno no meio da medida e vice-versa fazendo com que o sinal observado corresponda à média em $\theta$. Em uma análise inicial, vamos supor que temos controle sobre a fase $\theta$, portanto, as quantidades

\footnotetext{
${ }^{10}$ No nosso experimento, a precisão em frequência é da ordem de $\mathrm{mHz}$.

${ }^{11}$ Esta escala de tempo, para o laser que usamos no experimento, é da ordem de ms.
} 
calculadas em (3.58), (3.59) e (3.60) se tornam

$$
\begin{aligned}
\left\langle\hat{q}_{+}(\theta)^{2}\right\rangle & =\left\langle\hat{q}_{+}^{2}\right\rangle \cos ^{2} \theta+\left\langle\hat{p}_{-}^{2}\right\rangle \operatorname{sen}^{2} \theta-\left\langle\hat{q}_{+} \hat{p}_{-}\right\rangle \operatorname{sen}(2 \theta) \\
\left\langle\hat{p}_{+}(\theta)^{2}\right\rangle & =\left\langle\hat{p}_{+}^{2}\right\rangle \cos ^{2} \theta+\left\langle\hat{q}_{-}^{2}\right\rangle \operatorname{sen}^{2} \theta+\left\langle\hat{p}_{+} \hat{q}_{-}\right\rangle \operatorname{sen}(2 \theta) \\
\left\langle\hat{q}_{+}(\theta) \hat{p}_{+}(\theta)\right\rangle_{S} & =\left\langle\hat{q}_{+} \hat{p}_{+}\right\rangle_{S} \cos ^{2} \theta-\left\langle\hat{q}_{-} \hat{p}_{-}\right\rangle_{S} \operatorname{sen}^{2} \theta-\frac{\left\langle\hat{p}_{+} \hat{p}_{-}\right\rangle-\left\langle\hat{q}_{+} \hat{q}_{-}\right\rangle}{2} \operatorname{sen}(2 \theta) \\
\left\langle\hat{p}_{-}(\theta)^{2}\right\rangle & =\left\langle\hat{p}_{-}^{2}\right\rangle \cos ^{2} \theta+\left\langle\hat{q}_{+}^{2}\right\rangle \operatorname{sen}^{2} \theta+\left\langle\hat{q}_{+} \hat{p}_{-}\right\rangle \operatorname{sen}(2 \theta) \\
\left\langle\hat{q}_{-}(\theta)^{2}\right\rangle & =\left\langle\hat{q}_{-}^{2}\right\rangle \cos ^{2} \theta+\left\langle\hat{p}_{+}^{2}\right\rangle \operatorname{sen}^{2} \theta-\left\langle\hat{q}_{-} \hat{p}_{+}\right\rangle \operatorname{sen}(2 \theta) \\
\frac{\left\langle\hat{q}_{-}(\theta) \hat{p}_{-}(\theta)\right\rangle_{S}}{\left\langle\hat{p}_{+}(\theta) \hat{p}_{-}(\theta)\right\rangle-\left\langle\hat{q}_{+}(\theta) \hat{q}_{-}(\theta)\right\rangle} & \left\langle\hat{q}_{-} \hat{p}_{-}\right\rangle_{S} \cos ^{2} \theta-\left\langle\hat{q}_{+} \hat{p}_{+}\right\rangle_{S} \operatorname{sen}^{2} \theta-\frac{\left\langle\hat{p}_{+} \hat{p}_{-}\right\rangle-\left\langle\hat{q}_{+} \hat{q}_{-}\right\rangle}{2} \operatorname{sen}(2 \theta)
\end{aligned}
$$

onde o subscrito $S$ representa a correlação calculada em ordem simétrica. Se temos controle da fase $\theta$, a informação obtida com a dupla demodulação é exatamente a mesma que pode ser extraída variando ambas $\phi$ e $\theta$, como pode ser visto em 3.62 . 3.68). Entretanto, mesmo com este grau de liberdade adicional, a detecção homodina espectral ainda é insensível à quantidade $\delta_{\mathfrak{u}}(\theta=0)$.

A fase $\theta$ não é necessariamente estabilizada no experimento e pode sofrer um processo de difusão como no movimento browniano. Quando podemos garantir que esta fase é estável na escala de tempo da medida, $\delta \hat{i}_{o u t}^{c}$ e $\delta \hat{i}_{\text {out }}^{s}$ nos permite caracterizar completamente os subespaços de soma e subtração de maneira independente. Porém, quando há uma difusão de fase, estas informações são misturadas. Como

$$
\int_{0}^{2 \pi} d \theta \mathrm{e}^{2 i \theta}=0
$$

os ruídos calculados com as componentes seno e cosseno são iguais à parte real do espectro de ruído de uma fotocorrente estacionária

$$
\begin{aligned}
\int_{0}^{2 \pi} d \theta\left\langle\delta \hat{i}_{\text {out }}^{c} \delta \hat{i}_{\text {out }}^{c}\right\rangle=\int_{0}^{2 \pi} d \theta\left\langle\delta \hat{i}_{\text {out }}^{s} \delta \hat{i}_{\text {out }}^{s}\right\rangle & =\frac{1}{2}\left[\left\langle\delta \hat{i}_{\text {out }}^{c} \delta \hat{i}_{\text {out }}^{c}\right\rangle+\left\langle\delta \hat{i}_{\text {out }}^{s} \delta \hat{i}_{\text {out }}^{s}\right\rangle\right] \\
& \sim \operatorname{Re}\left[\left\langle\delta \hat{q}_{\phi}\left(\Omega_{\mathrm{an}}\right) \delta \hat{q}_{\phi}\left(-\Omega_{\mathrm{an}}\right)\right\rangle\right]
\end{aligned}
$$

e a correlação entre as componentes seno e cosseno se anulam

$$
\int_{0}^{2 \pi} d \theta\left\langle\delta \hat{i}_{\text {out }}^{c} \delta \hat{i}_{\text {out }}^{s}\right\rangle \sim \operatorname{Im}\left[\left\langle\delta \hat{q}_{\phi}\left(\Omega_{\mathrm{an}}\right) \delta \hat{q}_{\phi}\left(-\Omega_{\mathrm{an}}\right)\right\rangle\right]=0
$$

Para obter informação sobre a estacionariedade da fotocorrente, é necessário garan- 
tir uma boa estabilidade entre as fases da fotocorrente e do oscilador local eletrônico. Se esta estabilidade for suficiente para garantir que a fase $\theta$ não varia durante a aquisição de dados, a diferença entre os ruídos (3.48) e (3.56), assim como a correlação (3.57), nos dará a informação sobre a estacionariedade. Caso a estabilização não seja boa o suficiente, as medidas sempre irão parecer resultado de uma fotocorrente estacionária devido à difusão de fase. Neste sentido, o controle independente da fase do osciladores locais eletrônicos é importante para refutar a hipótese de estacionariedade da fotocorrente. Por outro lado, se assumimos que a fotocorrente é estacionária, a informação obtida usando a dupla demodulação - com fase relativa de $90^{\circ}$ entre os osciladores locais eletrônicos, mas sem controle da fase absoluta - é a mesma que obteríamos utilizando apenas um oscilador local eletrônico com controle da fase absoluta. De fato, a fase o oscilador local eletrônico está diretamente associada à evolução livre os operadores nas bandas laterais, pois as bandas laterais inferior e superior irão receber fases diferentes, $\hat{a}_{ \pm \Omega} \rightarrow \hat{a}_{\Omega} \mathrm{e}^{\mp i \Omega \frac{d}{c}}$, após se propagar por uma distância $d$. No espaço de soma e subtração de bandas laterais, esta evolução se torna

$$
\hat{a}_{+}(\theta)=\cos (\theta) \hat{a}_{+}+i \operatorname{sen}(\theta) \hat{a}_{-}, \quad \text { e } \quad \hat{a}_{-}(\theta)=\cos (\theta) \hat{a}_{-}-i \operatorname{sen}(\theta) \hat{a}_{+},
$$

onde $\theta=\Omega d / c$. Note que esta é o mesmo operador a que temos acesso se tivermos controle da fase $\theta$ entre o oscilador local eletrônico e a banda lateral. Neste sentido, se $\Omega$ é muito bem calibrado, este controle adicional da fase $\theta$ fornece uma medida indireta da distância de propagação da luz.

\subsection{Detecção com Cavidade}

Como discutido antes, para medir a informação presente na quadratura fase, é necessário convertê-la para a quadratura amplitude. Usando a detecção homodina, uma fase controlável é introduzida entre a portadora do campo de referência e as bandas laterais do campo de interesse. Essa conversão é, em última instância, resultado de um processo de interferência. Na detecção homodina, a fase introduzida é resultado de uma diferença de caminho entre os campos. Outra forma de fazer esta conversão é utilizar cavidades óticas para introduzir essa diferença de fase entre portadora e bandas laterais do mesmo campo. Ao ser refletido por uma cavidade vazia, o campo incidente é atenuado e sofre uma defasagem de $2 \pi$ ao passar pela ressonância. Esta técnica é muito prática pois, no caso do campo de interesse possuir uma portadora, não é necessário um campo de referência. Neste caso, o oscilador local ótico é a própria 
portadora.

Em uma cavidade Fabry-Perot, o campo que oscila na frequência $\omega_{p}$ refletido é dado por

$$
\alpha_{R}=r\left(\tilde{\Delta}_{p}\right) \alpha_{i n}, \quad \operatorname{com} \quad r\left(\tilde{\Delta}_{p}\right)=\frac{\sqrt{R}-\sqrt{R^{\prime}} \mathrm{e}^{\frac{\tilde{i} \Delta_{p}}{\mathcal{F}}}}{1-\sqrt{R R^{\prime}} \mathrm{e}^{\frac{i \Delta_{p}}{\mathcal{F}}}} .
$$

como em (2.19) e (2.27), quando há campo incidente em apenas uma das entradas. A quantidade $\widetilde{\Delta}_{p}$ é a dessintonia do campo em relação à frequência ressonância mais próxima de $\omega_{p}$ em unidades de largura de banda, $\mathcal{F}$ é a finesse da cavidade e $R$ e $R^{\prime}$ são os coeficientes de reflexão dos espelhos. Se houver luz entrando pelo outro espelho, os coeficientes de reflexão e transmissão são calculados fazendo $r \leftrightarrow r^{\prime}$. Devido ao princípio da superposição, a cavidade Fabry-Perot na qual há luz entrando por ambos espelhos pode ser dividida em duas, cada uma com entradas somente em um dos espelhos. Neste caso, o campo mostrado em 3.73 será acrescido do campo transmitido pela cavidade devido à luz incidente no segundo espelho como

$$
\alpha_{R}=r\left(\widetilde{\Delta}_{p}\right) \alpha_{i n}+t\left(\widetilde{\Delta}_{p}\right) \alpha_{v}, \quad \operatorname{com} \quad t\left(\widetilde{\Delta}_{p}\right)=\frac{\sqrt{T T^{\prime}} \mathrm{e}^{i \frac{\tilde{\eta} \tilde{\Delta}_{p}}{\mathcal{F}}}}{1-\sqrt{R R^{\prime}} \mathrm{e}^{i \frac{\tilde{\Delta}_{p}}{\mathcal{F}}}},
$$

onde $\eta=L_{\mathrm{e}} / L$ como mostrado em (2.22). Podemos olhar os campos clássicos $\alpha_{i n}$ e $\alpha_{v}$ como sendo os valores médios dos operadores de campo como mostrado em (3.16). Os operadores temporais não vão satisfazer a mesma equação, pois são compostos por operadores que atuam em modos de diversas frequências. Entretanto, cada modo de frequência bem definida irá obedecer (3.74),

$$
\hat{a}_{\omega}^{R}=r(\omega) \hat{a}_{\omega}^{i n}+t(\omega) \hat{a}_{\omega}^{v}
$$

No regime de alta finesse, os coeficientes de transmissão e reflexão se tornam

$$
r\left(\omega^{\prime}\right)=-\frac{\sqrt{R_{\min }}+2 i \omega^{\prime}}{1-2 i \omega^{\prime}}, t\left(\omega^{\prime}\right)=\frac{\sqrt{1-R_{\min }}}{1-2 i \omega^{\prime}} \operatorname{com} R_{\min }=\left(\frac{T-T^{\prime}}{T+T^{\prime}}\right)^{2}
$$

com $\omega^{\prime}$ representando $\omega$ normalizado pela largura de banda como em (2.28). Nestas condições, $R_{\min }$ é o único parâmetro que vai determinar o comportamento dos coeficientes $r$ e $t$.

O operador temporal formado pelo campo refletido é proporcional à integração em todas as componentes de frequências das bandas laterais, como em (3.14), e o operador 
associado à medida direta pelo fotodetector é como o mostrado em (3.18),

$$
\delta \hat{q}_{R}(t)=\delta \hat{a}_{R}(t)+\delta \hat{a}_{R}^{\dagger}(t) \quad \text { e } \quad \delta \hat{a}_{R}(t)=\int_{-\infty}^{+\infty} d \Omega \hat{a}_{\Omega}^{R} \mathrm{e}^{-i \Omega t}
$$

onde $\Omega=\omega-\omega_{p}$ são as frequências de batimento. Após a demodulação usando a componente cosseno como referência, o operador de medida será

$$
\delta \hat{i}_{R}^{c}(t)=\frac{K\left|\alpha_{R} \| g\right|}{2}\left[I_{0} \delta \hat{q}_{R}\left(-\Omega_{\mathrm{an}}\right)+I_{0}^{*} \hat{q}_{R}\left(\Omega_{\mathrm{an}}\right)\right] .
$$

onde $\delta \hat{q}_{R}\left(\Omega_{\mathrm{an}}\right)$ é o operador referente à transformada de Fourier de $\delta \hat{q}_{R}(t)$,

$$
\delta \hat{q}_{R}\left(\Omega_{\mathrm{an}}\right)=\frac{1}{\sqrt{2}}\left[\cos \left[\phi_{R}\left(\tilde{\Delta}_{p}\right)\right]\left(\hat{q}_{+}^{R}+i \hat{p}_{-}^{R}\right)+\operatorname{sen}\left[\phi_{R}\left(\widetilde{\Delta}_{p}\right)\right]\left(\hat{p}_{+}^{R}-i \hat{q}_{-}^{R}\right)\right] .
$$

Como definido em (3.15), $\hat{q}_{+}^{R}=\left[\hat{a}_{+}^{R}\right]^{\dagger}+\hat{a}_{+}^{R}, \hat{p}_{-}^{R}=i\left(\left[\hat{a}_{-}^{R}\right]^{\dagger}-\hat{a}_{-}^{R}\right)$ e $\phi_{R}\left(\tilde{\Delta}_{p}\right)$ é a fase introduzida pela cavidade entre as portadoras refletida e incidente. Esta expressão é similar à encontrada para a detecção homodina, com o diferencial que as bandas laterais, representadas pelos operadores $\hat{q}_{ \pm}^{R}$ e $\hat{p}_{ \pm}^{R}$, também são afetadas pela cavidade. Esta distinção está no cerne da vantagem que esta possui sobre a detecção homodina. Como o estado de vácuo tem valor médio nulo, a intensidade média refletida é função somente do campo de entrada, $\alpha_{R}=r\left(\tilde{\Delta}_{p}\right) \alpha_{i n}$, fazendo com que $\phi_{R}\left(\tilde{\Delta}_{p}\right)= \pm i \ln \left(\alpha_{R} /\left|\alpha_{R}\right|\right)= \pm i \ln \left(r\left(\tilde{\Delta}_{p}\right) /\left|r\left(\tilde{\Delta}_{p}\right)\right|\right)$. Os operadores de criação no espaço de soma e subtração das bandas laterais do campo refletido são

$$
\hat{a}_{ \pm}^{R}=\frac{\hat{a}_{\Omega^{\prime}}^{R} \pm \hat{a}_{-\Omega^{\prime}}^{R}}{\sqrt{2}}=\frac{1}{\sqrt{2}}\left[r\left(\widetilde{\Delta}_{p}+\Omega^{\prime}\right) \hat{a}_{\Omega}^{i n} \pm r\left(\widetilde{\Delta}_{p}-\Omega^{\prime}\right) \hat{a}_{-\Omega}^{i n}+t\left(\widetilde{\Delta}_{p}+\Omega^{\prime}\right) \hat{a}_{\Omega}^{v} \pm t\left(\tilde{\Delta}_{p}-\Omega^{\prime}\right) \hat{a}_{-\Omega}^{v}\right](3
$$

onde $\Omega^{\prime}$ é igual a $\Omega_{\text {an }}$ medida em unidades de largura de banda da cavidade. Consequentemente, as combinações de operadores hermitianos mostradas em (3.79) serão

$$
\begin{array}{r}
\hat{q}_{+}^{R}+i \hat{p}_{-}^{R}=A_{+}\left(\hat{q}_{+}^{i n}+i \hat{p}_{-}^{i n}\right)+A_{-}\left(\hat{p}_{+}^{i n}-i \hat{q}_{-}^{i n}\right)+B_{+}\left(\hat{q}_{+}^{v}+i \hat{p}_{-}^{v}\right)+B_{-}\left(\hat{p}_{+}^{v}-i \hat{q}_{-}^{v}\right) \\
\hat{p}_{+}^{R}-i \hat{q}_{-}^{R}=A_{+}\left(\hat{p}_{+}^{i n}-i \hat{q}_{-}^{i n}\right)-A_{-}\left(\hat{q}_{+}^{i n}+i \hat{p}_{-}^{i n}\right)+B_{+}\left(\hat{p}_{+}^{v}-i \hat{q}_{-}^{v}\right)-B_{-}\left(\hat{q}_{+}^{v}+i \hat{p}_{-}^{v}\right) \\
A_{+}=\frac{1}{2}\left[r\left(\tilde{\Delta}_{p}+\Omega^{\prime}\right)+r\left(\tilde{\Delta}_{p}-\Omega^{\prime}\right)^{*}\right], \quad A_{-}=\frac{i}{2}\left[r\left(\tilde{\Delta}_{p}+\Omega^{\prime}\right)-r\left(\widetilde{\Delta}_{p}-\Omega^{\prime}\right)^{*}\right], \\
B_{+}=\frac{1}{2}\left[t\left(\tilde{\Delta}_{p}+\Omega^{\prime}\right)-t\left(\tilde{\Delta}_{p}-\Omega^{\prime}\right)^{*}\right], \quad B_{-}=\frac{i}{2}\left[t\left(\tilde{\Delta}_{p}+\Omega^{\prime}\right)-t\left(\tilde{\Delta}_{p}-\Omega^{\prime}\right)^{*}\right] .
\end{array}
$$

Note que os operadores das bandas laterais do campo refletido são função dos operadores do campo incidente e do vácuo que incide no outro espelho da cavidade. Juntando estas expressões com (3.79) chegamos ao operador de medida para a detecção com 
cavidade,

$$
\begin{aligned}
& \delta \hat{q}_{R}(\Omega)=g_{x} \frac{\hat{q}_{+}^{i n}+i \hat{p}_{-}^{i n}}{\sqrt{2}}+g_{y} \frac{\hat{p}_{+}^{i n}-i \hat{q}_{-}^{i n}}{\sqrt{2}}+g_{x v} \frac{\hat{q}_{+}^{v}+i \hat{p}_{-}^{v}}{\sqrt{2}}+g_{y v} \frac{\hat{p}_{+}^{v}-i \hat{q}_{-}^{v}}{\sqrt{2}}, \\
& g_{x}\left(\widetilde{\Delta}_{p}, \Omega^{\prime}\right)=\frac{1}{2}\left[r\left(\tilde{\Delta}_{p}+\Omega^{\prime}\right) \mathrm{e}^{-i \phi_{R}\left(\tilde{\Delta}_{p}\right)}+r\left(\tilde{\Delta}_{p}-\Omega^{\prime}\right)^{*} \mathrm{e}^{i \phi_{R}\left(\tilde{\Delta}_{p}\right)}\right], \\
& g_{y}\left(\tilde{\Delta}_{p}, \Omega^{\prime}\right)=\frac{i}{2}\left[r\left(\tilde{\Delta}_{p}+\Omega^{\prime}\right) \mathrm{e}^{-i \phi_{R}\left(\tilde{\Delta}_{p}\right)}-r\left(\tilde{\Delta}_{p}-\Omega^{\prime}\right)^{*} \mathrm{e}^{i \phi_{R}\left(\tilde{\Delta}_{p}\right)}\right], \\
& g_{x v}\left(\tilde{\Delta}_{p}, \Omega^{\prime}\right)=\frac{1}{2}\left[t\left(\widetilde{\Delta}_{p}+\Omega^{\prime}\right) \mathrm{e}^{-i \phi_{R}\left(\tilde{\Delta}_{p}\right)}+t\left(\widetilde{\Delta}_{p}-\Omega^{\prime}\right)^{*} \mathrm{e}^{i \phi_{R}\left(\tilde{\Delta}_{p}\right)}\right], \\
& g_{y v}\left(\widetilde{\Delta}_{p}, \Omega^{\prime}\right)=\frac{i}{2}\left[t\left(\tilde{\Delta}_{p}+\Omega^{\prime}\right) \mathrm{e}^{-i \phi_{R}\left(\tilde{\Delta}_{p}\right)}-t\left(\tilde{\Delta}_{p}-\Omega^{\prime}\right)^{*} \mathrm{e}^{i \phi_{R}\left(\tilde{\Delta}_{p}\right)}\right],
\end{aligned}
$$

Como pode ser verificado diretamente nas expressões anteriores, os coeficientes $g_{x}$ e $g_{x v}$ são pares em relação à troca conjunta entre as bandas laterais, $\mp \Omega \rightarrow \pm \Omega$, e dessintonia $\widetilde{\Delta}_{p} \rightarrow-\widetilde{\Delta}_{p}$. De forma semelhante, $g_{y}$ e $g_{y v}$ são ímpares. Após a demodulação, o resultado medido nas componentes cosseno e seno são

$$
\begin{gathered}
\delta \hat{i}_{R}^{c} \sim \operatorname{Re}\left[g_{x}\right] \hat{q}_{+}^{i n}+\operatorname{Re}\left[g_{y}\right] \hat{p}_{+}^{i n}-\operatorname{Im}\left[g_{x}\right] \hat{p}_{-}^{i n}+\operatorname{Im}\left[g_{y}\right] \hat{q}_{-}^{i n}+\mathrm{vac}^{c}, \\
\delta \hat{i}_{R}^{s} \sim \operatorname{Re}\left[g_{x}\right] \hat{p}_{-}^{i n}-\operatorname{Re}\left[g_{y}\right] \hat{q}_{-}^{i n}+\operatorname{Im}\left[g_{x}\right] \hat{q}_{+}^{i n}+\operatorname{Im}\left[g_{y}\right] \hat{p}_{+}^{i n}+\mathrm{vac}^{s}, \\
\operatorname{vac}^{c}=\operatorname{Re}\left[g_{x v}\right] \hat{q}_{+}^{v}+\operatorname{Re}\left[g_{y v}\right] \hat{p}_{+}^{v}-\operatorname{Im}\left[g_{x v}\right] \hat{p}_{-}^{v}+\operatorname{Im}\left[g_{y v}\right] \hat{q}_{-}^{v}, \\
\operatorname{vac}^{s}=\operatorname{Re}\left[g_{x v}\right] \hat{p}_{-}^{v}-\operatorname{Re}\left[g_{y v}\right] \hat{q}_{-}^{v}+\operatorname{Im}\left[g_{x v}\right] \hat{q}_{+}^{v}+\operatorname{Im}\left[g_{y v}\right] \hat{p}_{+}^{v}
\end{gathered}
$$

Diferente do que ocorre na detecção homodina, a cavidade permite que mais correlações entre os subespaços de soma e subtração sejam medidas. Com a detecção com cavidades, é possível medir todas as entradas da matriz de covariâncias e, portanto, caracterizar por completo estados gaussianos nas bandas laterais.

Em uma primeira análise, para criar um pouco de intuição sobre as expressões 3.88 3.91), vamos tratar do caso particular em que a cavidade é fechada, ou seja, o coeficiente de transmissão se anula em todas as frequências, de modo que $t(x)=0$ e $r(x)=\mathrm{e}^{-i \phi(x)}$. Também vamos nos restringir ao caso em que a frequência de análise for bem menor que o intervalo espectral livre $(F S R)^{12}$ e bem maior que a largura de banda da cavidade ${ }^{13}$. Nestas circunstâncias, as três regiões de dessintonia mostradas na figura 3.11 serão interessantes: cavidade quase ressonante com a portadora e com as banda laterais superior e inferior. Na primeira região,

$$
g_{x} \approx \cos \left[\phi_{R}(\delta)\right], \quad g_{y} \approx \operatorname{sen}\left[\phi_{R}(\delta)\right], \quad \text { e } \quad g_{x v}=g_{y v}=0
$$

\footnotetext{
${ }^{12}$ Este é o caso no nosso experimento, onde $\Delta f \approx 1.8 \mathrm{GHz}$ e $\Omega_{\mathrm{an}}=21 \mathrm{MHz}$.

${ }^{13}$ Este não é o nosso caso, pois a largura de banda é de aproximadamente $15 \mathrm{MHz}$, mas ele será analisado mais adiante.
} 

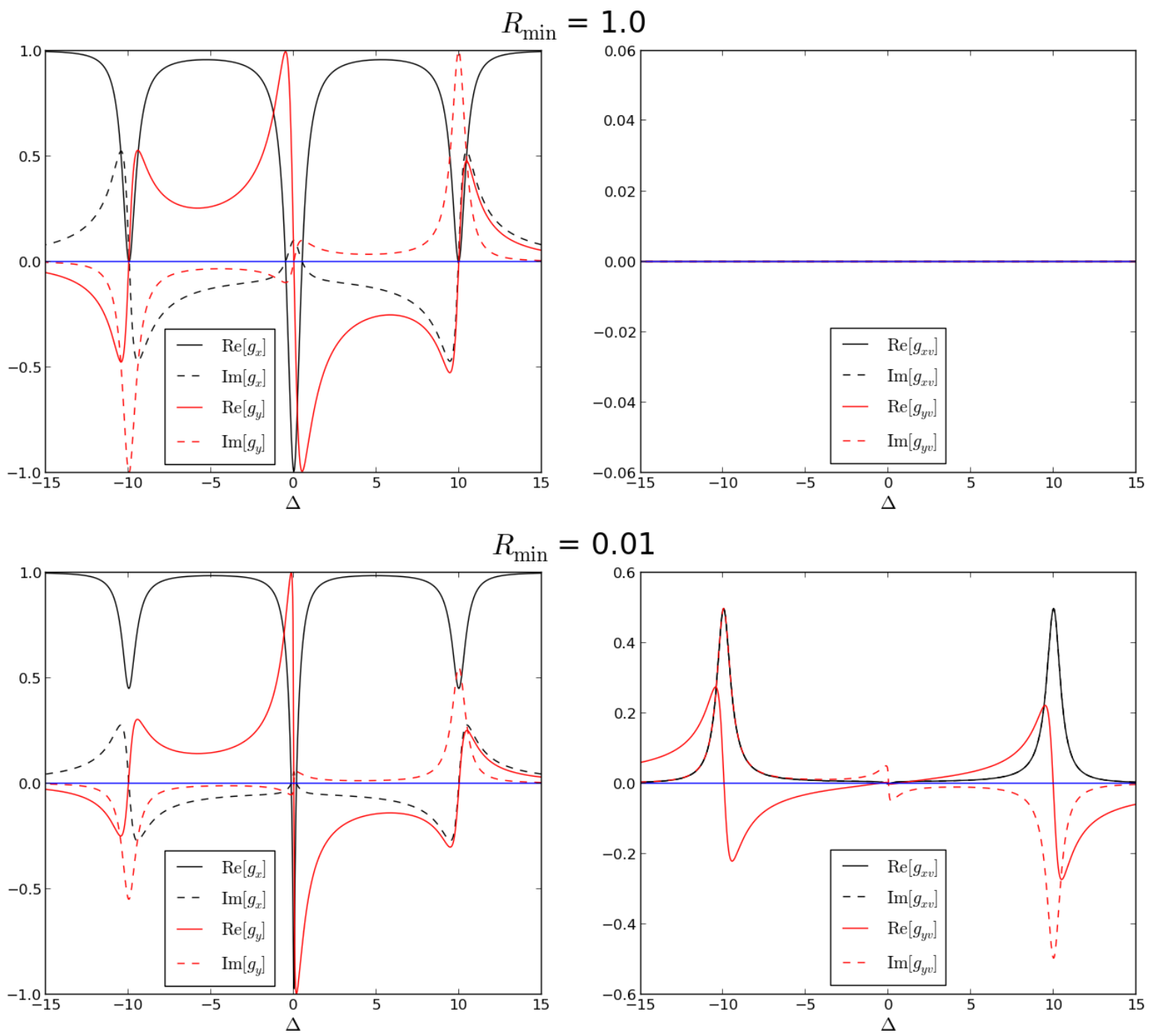

Figura 3.10: Coeficientes mostrados nas equações (3.88 3.91) Em duas situações extremas: cavidade fechada e próxima da situação de casamento de impedâncias. Note que a paridade em relação à dessintonia pode ser vista claramente em todos os gráficos.

onde $\delta$ é da ordem da largura de banda da cavidade. Estas conclusões vem do fato de que, como ambas as bandas laterais do campo incidente estão bem longe da ressonância, a cavidade não introduz nenhuma fase entre as mesmas e as do campo refletido. Neste caso, a cavidade realiza a mesma medida que a detecção homodina. Como pode ser visto explicitamente comparando (3.81), (3.82) e (3.79) com (3.14). Quando a cavidade está próxima da ressonância com uma das bandas laterais, $\widetilde{\Delta}_{p}=\delta \pm \Omega^{\prime}$ e

$$
\begin{gathered}
g_{x}\left(\delta \pm \Omega^{\prime}, \Omega^{\prime}\right)=\frac{1}{2}\left[r\left(\delta+2 \Omega^{\prime}\right)+r(\delta)^{*}\right] \approx \mathrm{e}^{ \pm i \frac{\phi(\delta)}{2}} \cos \left(\frac{\phi(\delta)}{2}\right), \\
g_{y}\left(\delta \pm \Omega^{\prime}, \Omega^{\prime}\right)=\frac{i}{2}\left[r\left(\delta+2 \Omega^{\prime}\right)-r(\delta)^{*}\right] \approx \mathrm{e}^{ \pm i \frac{\phi(\delta)}{2}} \operatorname{sen}\left(\frac{\phi(\delta)}{2}\right)
\end{gathered}
$$


e $g_{x v}=g_{y v}=0$. Em ambos os casos, a portadora está bem fora de ressonância com a cavidade, ou seja $\phi\left(\widetilde{\Delta}_{p}=\delta \pm \Omega\right) \approx 0$. Portanto, a expressão em 3.79 se torna

$$
\delta \hat{q}_{R}\left(\Omega_{\mathrm{an}}\right)=\frac{1}{\sqrt{2}}\left[\hat{q}_{+}^{R}+i \hat{p}_{-}^{R}\right]=\frac{\mathrm{e}^{\mp i \frac{\phi(\delta)}{2}}}{\sqrt{2}}\left[\cos \left(\frac{\phi(\delta)}{2}\right)\left(\hat{q}_{+}^{i n}+i \hat{p}_{-}^{i n}\right)+\operatorname{sen}\left(\frac{\phi(\delta)}{2}\right)\left(\hat{p}_{+}^{i n}-i \hat{q}_{-}^{i n}\right)\right],
$$

para $\widetilde{\Delta}_{p}=\delta \pm \Omega^{\prime}$. Esta expressão também é mesma encontrada na detecção homodina a menos da fase global de $\pm \phi(\delta) / 2$ - que pode ser tomada como nula sem perda de generalidade, pois é equivalente a incluí-la na fase da portadora - e o fator 2 na rotação. Como a cavidade introduz uma rotação completa ao passar pela ressonância, a medida em cada banda lateral é capaz de varrer somente metade do espaço de parâmetros em comparação com a rotação na portadora. Em outras palavras, quando a cavidade passa pela ressonância com a portadora, é introduzida uma fase que varia entre 0 e $2 \pi$ e para cada uma das bandas laterais, ocorre apenas uma rotação entre 0 e $\pi$ Neste sentido, a informação adquirida pela rotação das bandas laterais permite acessar de forma redundante metade da informação acessada usando a portadora. Em uma cavidade fechada, a rotação de fase introduzida pela cavidade tanto na portadora quanto nas bandas laterais é equivalente à obtida com a detecção homodina. Esta
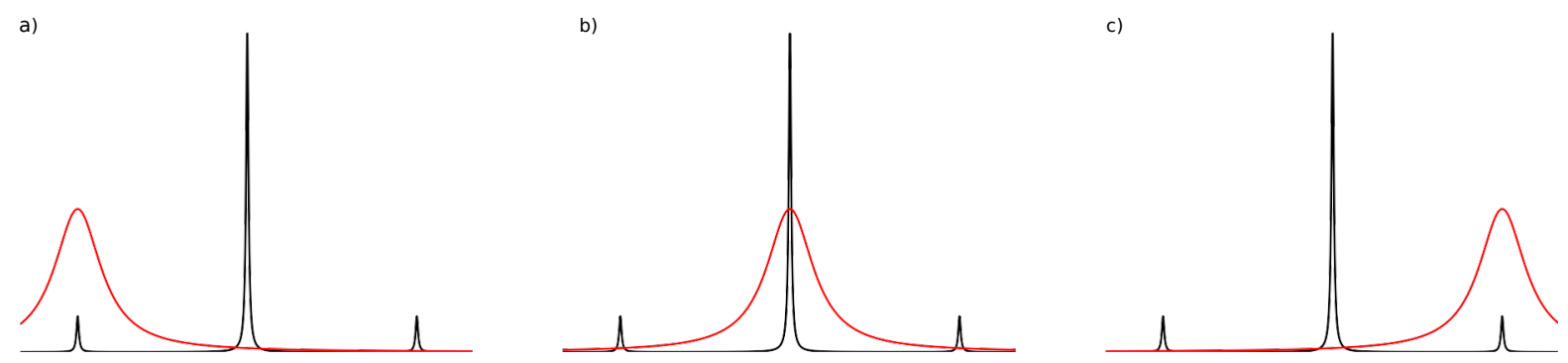

Figura 3.11: Varredura da cavidade: as curvas pretas representam a portadora e suas bandas laterais (as bandas laterais estão grandes na figura, mas no nosso experimento elas são apenas ruídos) e as curvas vermelhas representam um modo normal da cavidade. As três regiões principais são mostradas na figura. A região representada em a) é tal que a ressonância da cavidade está em torno da banda lateral inferior. Na região b), a ressonância da cavidade está próxima da frequência portadora e na região c), da banda lateral superior.

última conclusão é válida somente para a cavidade fechada. Neste tipo de sistema, as bandas laterais não são atenuadas ao passar pela ressonância, por isso a medida não introduz desbalanceamento de energia entre as mesmas. Desta forma, é de se esperar que a entrada da matriz de covariâncias que mede o desbalanceamento de energia, $\left\langle\hat{q}_{+} \hat{q}_{-}+\hat{p}_{+} \hat{p}_{-}\right\rangle$, não possa ser observada em uma cavidade fechada. Entretanto, em cavidades reais, que possuem perdas, uma das bandas laterais é naturalmente 
atenuada quando está em ressonância com a cavidade e este efeito é maior quanto mais próxima da condição de casamento de impedâncias a cavidade estiver.

Para a cavidade aberta, a rotação devido à portadora ocorre como em (3.92), pois os coeficientes de transmissão serão nulos para ambas as bandas laterais, que estão fora de ressonância, e a fase introduzida pela cavidade na reflexão também se anula. Quando a cavidade está próxima da ressonância com as bandas laterais, os coeficientes $g_{x}$ e $g_{y}$ se tornam

$$
g_{x}\left(\delta \pm \Omega, \Omega^{\prime}\right) \approx 1+|r(\delta)| \mathrm{e}^{ \pm i \phi(\delta)}, \quad g_{y}\left(\delta \pm \Omega, \Omega^{\prime}\right) \approx \pm i\left[1-|r(\delta)| \mathrm{e}^{ \pm i \phi(\delta)}\right]
$$

e os coeficientes $g_{x v}$ e $g_{y v}$ não se anulam. Note que as expressões acima convergem para 3.93 e (3.94) quando $|r(\delta)|=1$. Neste caso, os coeficientes $g_{x}$ e $g_{y}$ possuem uma parte imaginária, que irá misturar os subespaços de soma e subtração.

Como discutido anteriormente, a detecção homodina é incapaz de caracterizar por completo estados gaussianos das bandas laterais. O espectro de ruído de intensidade do campo refletido pela cavidade é $S q_{R}\left(\Omega_{\mathrm{an}}\right)=\left\langle\delta \hat{q}_{R}\left(\Omega_{\mathrm{an}}\right) \delta \hat{q}_{R}\left(-\Omega_{\mathrm{an}}\right)\right\rangle \mathrm{e}$

$$
\begin{aligned}
S q_{R}\left(\Omega_{\mathrm{an}}\right)= & A_{x x} \frac{\left\langle\hat{q}_{+}^{2}\right\rangle+\left\langle\hat{p}_{-}^{2}\right\rangle}{2}+A_{y y} \frac{\left\langle\hat{p}_{+}^{2}\right\rangle+\left\langle\hat{q}_{-}^{2}\right\rangle}{2}+2 \operatorname{Re}\left[A_{x y}\right] \frac{\left\langle\hat{q}_{+} \hat{p}_{+}\right\rangle_{S}-\left\langle\hat{q}_{-} \hat{p}_{-}\right\rangle_{S}}{2} \\
& -2 \operatorname{Im}\left[A_{x y}\right] \frac{\left\langle\hat{q}_{+} \hat{q}_{-}\right\rangle+\left\langle\hat{p}_{+} \hat{p}_{-}\right\rangle}{2}+\left|g_{x v}\right|^{2}+\left|g_{y v}\right|^{2}
\end{aligned}
$$

com $A_{x y}\left(\Delta_{p}, \Omega^{\prime}\right)=g_{x}\left(\Delta_{p}, \Omega^{\prime}\right) g_{y}\left(\Delta_{p}, \Omega^{\prime}\right)^{*}$, tal que o mesmo vale para $A_{x x}$ e $A_{y y}$.

Os coeficientes referentes ao vácuo transmitido pela cavidade estão relacionados com os mostrados na figura 7.15 pela relação $\left|g_{x v}\right|^{2}+\left|g_{y v}\right|^{2}=1-A_{x x}-A_{y y}$, que é uma consequência direta do caso particular em que as bandas laterais do campo incidente também estão no estado de vácuo. Na situação em que a fotocorrente é estacionária, o espectro de ruído do campo refletido fornece toda a informação sobre a matriz de covariâncias das bandas laterais através da relação (3.97). Esta conclusão é válida também quando a fotocorrente não é estacionária, porém é no caso estacionário que há o grande diferencial da detecção com cavidades, pois a detecção homodina não é sensível a $\delta_{u}(\theta)$. Por outro lado, todas as entradas da matriz de covariâncias que se anulam no caso estacionário podem ser medidas tanto pela detecção homodina quanto pela detecção com cavidades. Nestas circunstâncias, podemos reescrever a expressão 3.97 como

$$
S q_{R}\left(\Omega_{\mathrm{an}}\right)=A_{x x}(\alpha-1)+A_{y y}(\beta-1)+2 \operatorname{Re}\left[A_{x y}\right] \gamma-2 \operatorname{Im}\left[A_{x y}\right] \delta_{\mathbf{u}}+1
$$



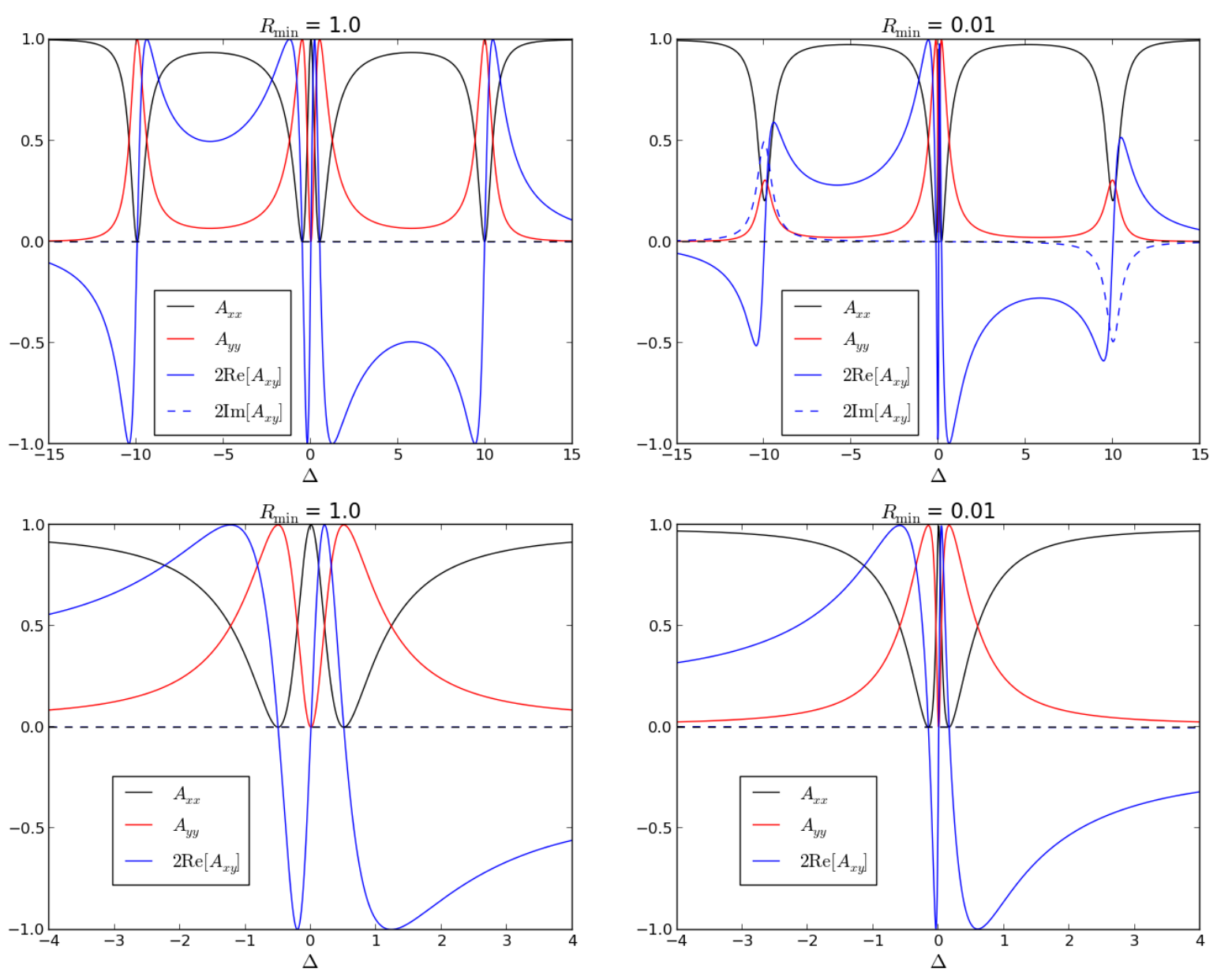

Figura 3.12: Coeficientes mostrados na equação (3.97). Os gráficos à esquerda representam a uma situação em que a cavidade é fechada e os da direita próxima da situação de casamento de impedâncias. Note que todos estes coeficientes são bem distintos e podem ser utilizados em um procedimento de ajuste, como o de mínimos quadrados, para inferir os valores de $\alpha, \beta, \gamma$ e $\delta_{\mathrm{u}}$.

onde $\alpha=\left\langle\hat{q}_{+}^{2}\right\rangle=\left\langle\hat{p}_{-}^{2}\right\rangle, \beta=\left\langle\hat{p}_{+}^{2}\right\rangle=\left\langle\hat{q}_{-}^{2}\right\rangle, \gamma=\left\langle\hat{q}_{+} \hat{p}_{+}\right\rangle_{S}=-\left\langle\hat{q}_{-} \hat{p}_{-}\right\rangle_{S}$ e $\delta_{\mathrm{u}}=\left\langle\hat{q}_{+} \hat{q}_{-}\right\rangle=\left\langle\hat{p}_{+} \hat{p}_{-}\right\rangle$. Estas quantidades são os parâmetros do espectro de ruído do campo de entrada e podem ser arranjadas das seguintes maneiras

$$
S_{i n}\left(\Omega_{\mathrm{an}}\right)=\left(\begin{array}{cc}
\alpha & \gamma+i \delta_{\mathrm{u}} \\
\gamma-i \delta_{\mathrm{u}} & \beta
\end{array}\right) \text { e } V_{+/-}=\left(\begin{array}{cccc}
\alpha & \gamma & \delta_{\mathrm{u}} & 0 \\
\gamma & \beta & 0 & \delta_{\mathrm{u}} \\
\delta_{\mathrm{u}} & 0 & \beta & -\gamma \\
0 & \delta_{\mathrm{u}} & -\gamma & \alpha
\end{array}\right) \text {, }
$$

onde $V_{+/-}=\left\langle\hat{X} \hat{X}^{T}\right\rangle \operatorname{com} \hat{X}=\left(\hat{q}_{+}, \hat{p}_{+}, \hat{q}_{-}, \hat{p}_{-}\right)$

O coeficiente $\operatorname{Im}\left[A_{x y}\right]$, que permite acessar o termo $\delta_{\mathrm{u}}$, se anula quando $R_{\min }=1.0$ e nas proximidades da ressonância com a portadora. Ele somente se torna importante quando a cavidade está próxima da ressonância com umas bandas laterais, pois nesta 
situação uma fração da luz é transmitida pela cavidade. O mesmo ocorre com o vácuo que incide no outro espelho de modo que esta fração é maior quanto mais próxima a cavidade está da condição de casamento de impedâncias. Consequentemente, o campo refletido pela cavidade carrega a informação do campo incidente em uma das bandas laterais e a do vácuo na outra. Esta assimetria é o que possibilita a medida do termo $\delta_{\mathrm{u}}$.

\subsection{Generalização para Mais de um Campo}

Até o momento restringi a discussão a apenas o caso em que uma portadora e suas respectivas bandas laterais incidem sobre cada detector. Como discutirei em seguida este é o único caso importante, pois para analisar um sistema composto formado por mais de um campo basta enviar cada um deles para um detector e fazer a medida conjunta de quadratura. Considere que agora temos $n$ portadoras, cada uma com suas respectivas bandas laterais. O espaço de Hilbert deste sistema agora se torna

$$
\mathcal{H}_{p}=\mathcal{H}_{p 1} \otimes \mathcal{H}_{p 2} \otimes \ldots \otimes \mathcal{H}_{p n} \quad \text { e } \quad \mathcal{H}_{B L}=\mathcal{H}_{B L 1} \otimes \mathcal{H}_{B L 1} \otimes \ldots \otimes \mathcal{H}_{B L n}
$$

Como discutido na seção 3.1, a medida que fazemos é insensível a correlações entre bandas laterais e portadora do mesmo campo, pelo fato de a portadora ser parte integrante do aparato experimental usado na medida. Isto faz com que, no caso de mais de um campo, também não seja possível medir correlações entre as portadoras. Por este motivo, podemos considerar, sem perda de generalidade, o estado do sistema conjunto como

$$
\hat{\rho}=\hat{\rho}_{p} \otimes \hat{\rho}_{\mathrm{BL}}, \quad \text { com } \quad \hat{\rho}_{p}=\bigotimes_{i=1}^{n} \hat{\rho}_{p i} .
$$

\subsubsection{Detecção Homodina}

Toda a descrição da medida das flutuações de intensidade mostrada no decorrer deste capítulo pode ser aplicada para o caso com mais de um campo desde que apenas portadoras de mesma frequência sejam enviadas a cada detector. Se duas portadoras com exatamente a mesma frequência são enviadas a um determinado detector, iremos medir, além do sinal de cada uma delas, um termo de interferência no sinal DC. As bandas laterais também sofrem interferência e o sinal medido pelo detector será similar 


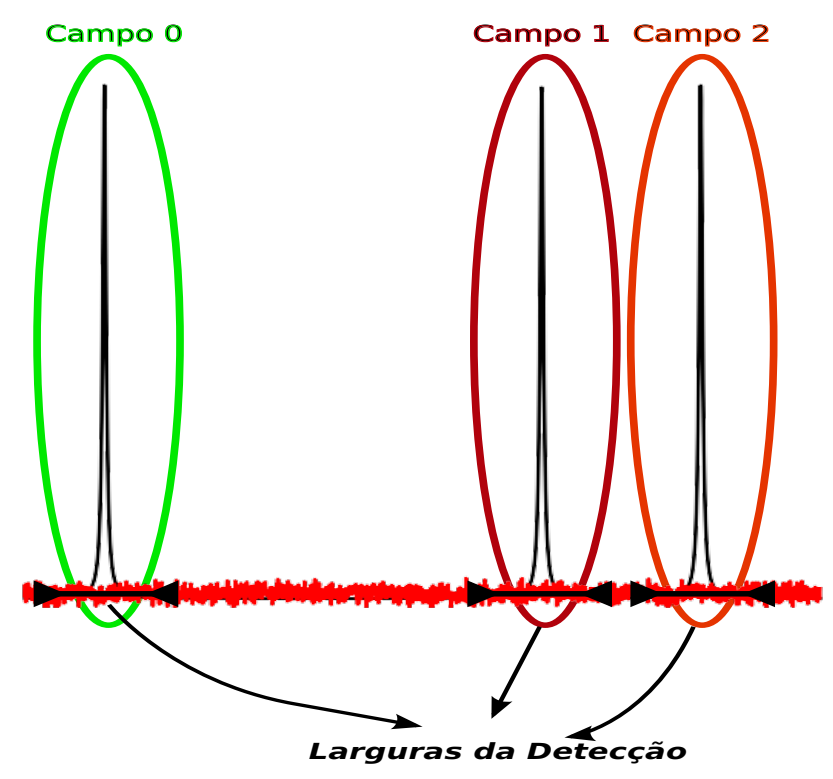

Figura 3.13: Densidade espectral de diversos campos na condição em que cada portadora possui frequências bem distintas e acima da largura de detecção eletrônica.

aos mostrados em (3.21) e (3.23). Ele é uma mistura das bandas laterais de cada campo amplificadas por suas respectivas portadoras com as mesmas bandas laterais amplificadas pela portadora do outro campo,

$$
\delta \hat{I}(t)=\sqrt{K}|g|\left[\left|\alpha_{1}\right| \delta \hat{q}_{1}(t)+\left|\alpha_{2}\right| \delta \hat{q}_{2}(t)+\left|\alpha_{1}\right| \delta \hat{q}_{2 \phi}(t)+\left|\alpha_{2}\right| \delta \hat{q}_{1-\phi}(t)\right],
$$

onde $\left|\alpha_{i}\right|^{2}$ é proporcional à intensidade média de cada portadora e $\phi$ é a fase entre ambas. Isto é exatamente o que ocorre na detecção homodina. Por outro lado, se as duas portadoras possuem frequências distintas duas situações podem ocorrer: 1 a frequência do batimento entre as portadoras estará na mesma faixa de frequência das bandas laterais, o que irá adulterar a medida das mesmas ${ }^{14}$, 2 - a frequência do batimento se encontra fora da faixa de frequência das bandas laterais, o que fará com que e o sinal das bandas laterais referentes às duas portadoras sejam misturadas de tal maneira que é muito difícil separá-las novamente. Neste caso, a análise do estado das bandas laterais está fora do escopo deste trabalho.

Se usássemos detecções homodinas independentes para cada um dos campos, seríamos capazes de medir diretamente o operador $\delta \hat{q}_{i \phi_{i}}(t)$, com $\phi_{i}$ igual à fase relativa entre o campo de interesse e o oscilador local ótico, como em 3.23. Podemos acessar o domínio da frequência fazendo demodulações com as componentes seno e cosseno dos osciladores locais eletrônicos em cada campo. Os sinais de saída da

\footnotetext{
${ }^{14} \mathrm{~A}$ medida do valor médio neste caso é a base de uma técnica conhecida como detecção heterodina.
} 
demodulação do campo $i$ são $\left(\delta \hat{i}_{\text {out }}^{c}\right)_{i}$ e $\left(\delta \hat{i}_{\text {out }}^{\text {s }}\right)_{i}$ e são iguais a

$$
\left(\delta \hat{i}_{\text {out }}^{c}\right)_{i}=\hat{q}_{i+}\left(\theta_{i}\right) \cos \left(\phi_{i}\right)+\hat{p}_{i+}\left(\theta_{i}\right) \operatorname{sen}\left(\phi_{i}\right) \text { e }\left(\delta \hat{i}_{\text {out }}^{s}\right)_{i}=\hat{p}_{i-}\left(\theta_{i}\right) \cos \left(\phi_{i}\right)-\hat{q}_{i-}\left(\theta_{i}\right) \operatorname{sen}\left(\phi_{i}\right),
$$

de forma similar a (3.46) e (3.52). Consequentemente somos capazes de observar o operador de quadratura espectral para as bandas laterais de cada campo $i$ de forma similar a (3.14),

$$
\sqrt{2} \delta \hat{q}_{i \phi_{i}}(\Omega)=\left(\delta \hat{i}_{\text {out }}^{c}\right)_{i}+i\left(\delta \hat{i}_{\text {out }}^{s}\right)_{i} .
$$

Se fizéssemos uma medida conjunta dos campos $i$ e $j$, seria possível medir toda e qualquer correlação presente entre eles, pois é possível variar as fases $\phi_{i}$ e $\phi_{j}$ de maneira independente, por meio da tomografia homodina [25]. Mais especificamente, podemos medir a parte real (ou imaginária) de $\delta \hat{q}_{i \phi_{i}}(\Omega)$ e fazer a medida conjunta com a parte real (ou imaginária) de $\delta \hat{q}_{j \phi_{j}}(\Omega)$. Este procedimento permite medir o estado conjunto de qualquer combinação dos modos $\hat{a}_{i \pm}$ e $\hat{a}_{j \pm}$. Porém ainda não seríamos capazes de medir todas as correlações entre $\hat{a}_{i+}$ e $\hat{a}_{i-}$ ou $\hat{a}_{j+}$ e $\hat{a}_{j-}$, mesmo em estados gaussianos, pelos motivos apresentados na seção 3.4 .4 .

\subsubsection{Detecção com Cavidades}

Na detecção com cavidades, cada componente de frequência da matriz espectral será transformada como mostrado em (3.83),

$$
\delta \hat{q}_{i R}(\Omega)=g_{x}^{i} \frac{\hat{q}_{i+}^{i n}+i \hat{p}_{i-}^{i n}}{\sqrt{2}}+g_{y}^{i} \frac{\hat{p}_{i+}^{i n}-i \hat{q}_{i-}^{i n}}{\sqrt{2}}+g_{x v}^{i} \frac{\hat{q}_{i+}^{v}+i \hat{p}_{i-}^{v}}{\sqrt{2}}+g_{y v}^{i} \frac{\hat{p}_{i+}^{v}-i \hat{q}_{i-}^{v}}{\sqrt{2}} .
$$

Como discutido anteriormente, a detecção com cavidades é equivalente à detecção homodina quando somente a rotação da portadora é levada em consideração. Também como discutido antes, a detecção homodina é capaz de fazer a reconstrução completa do estado conjunto dos modos de soma e subtração das bandas laterais envolvendo campos distintos. Deste modo, podemos concluir que a detecção com cavidades é capaz de caracterizar qualquer tipo de estado conjunto entre os modos presentes em campos diferentes desde que possa separar a ressonância da portadora das bandas laterais.

Para estados gaussianos, toda a informação com respeito ao emaranhamento está contida nos segundos momentos. Os espectros de ruído e correlações espectrais estão relacionados aos ruídos e correlações de $\left(\delta \hat{i}_{\text {out }}^{c}\right)_{i}$ e $\left(\delta \hat{i}_{\text {out }}^{s}\right)_{i}$ da mesma forma que em 3.48 . 
3.56, 3.57). As correlações espectrais entre os campos $i$ e $j$ são dados por

$$
\begin{aligned}
& \left\langle\left(\delta \hat{i}_{\text {out }}^{c}\right)_{i}\left(\delta \hat{i}_{\text {out }}^{c}\right)_{j}\right\rangle \sim \operatorname{Re}\left[\mathrm{e}^{i\left(\theta_{i}-\theta_{j}\right)}\left\langle\delta \hat{q}_{i}\left(\Omega_{\mathrm{an}}\right) \delta \hat{q}_{j}\left(-\Omega_{\mathrm{an}}\right)\right\rangle\right]+\operatorname{Re}\left[\mathrm{e}^{i\left(\theta_{i}+\theta_{j}\right)}\left\langle\delta \hat{q}_{i}\left(\Omega_{\mathrm{an}}\right) \delta \hat{q}_{j}\left(\Omega_{\mathrm{an}}\right)\right\rangle\right], \\
& \left\langle\left(\delta \hat{i}_{\text {out }}^{\mathrm{s}}\right)_{i}\left(\delta \hat{i}_{\text {out }}^{\mathrm{s}}\right)_{j}\right\rangle \sim \operatorname{Re}\left[\mathrm{e}^{i\left(\theta_{i}-\theta_{j}\right)}\left\langle\delta \hat{q}_{i}\left(\Omega_{\mathrm{an}}\right) \delta \hat{q}_{j}\left(-\Omega_{\mathrm{an}}\right)\right\rangle\right]-\operatorname{Re}\left[\mathrm{e}^{i\left(\theta_{i}+\theta_{j}\right)}\left\langle\delta \hat{q}_{i}\left(\Omega_{\mathrm{an}}\right) \delta \hat{q}_{j}\left(\Omega_{\mathrm{an}}\right)\right\rangle\right], \\
& \left\langle\left(\delta \hat{i}_{\text {out }}^{c}\right)_{i}\left(\delta \hat{i}_{\text {out }}^{\mathrm{s}}\right)_{j}\right\rangle \sim \operatorname{Im}\left[\mathrm{e}^{i\left(\theta_{i}-\theta_{j}\right)}\left\langle\delta \hat{q}_{i}\left(\Omega_{\mathrm{an}}\right) \delta \hat{q}_{j}\left(-\Omega_{\mathrm{an}}\right)\right\rangle\right]+\operatorname{Im}\left[\mathrm{e}^{i\left(\theta_{i}+\theta_{j}\right)}\left\langle\delta \hat{q}_{i}\left(\Omega_{\mathrm{an}}\right) \delta \hat{q}_{j}\left(\Omega_{\mathrm{an}}\right)\right\rangle\right], \\
& \left\langle\left(\delta \hat{i}_{\text {out }}^{\mathrm{s}}\right)_{i}\left(\delta \hat{i}_{\text {out }}^{c}\right)_{j}\right\rangle \sim \operatorname{Im}\left[\mathrm{e}^{i\left(\theta_{i}-\theta_{j}\right)}\left\langle\delta \hat{q}_{i}\left(\Omega_{\mathrm{an}}\right) \delta \hat{q}_{j}\left(-\Omega_{\mathrm{an}}\right)\right\rangle\right]-\operatorname{Im}\left[\mathrm{e}^{i\left(\theta_{i}+\theta_{j}\right)}\left\langle\delta \hat{q}_{i}\left(\Omega_{\mathrm{an}}\right) \delta \hat{q}_{j}\left(\Omega_{\mathrm{an}}\right)\right\rangle\right],
\end{aligned}
$$

onde já considero as fases $\phi_{i}=\phi_{j}=0$, pois neste caso estamos fazendo a medida direta no fotodetector, sem a detecção homodina. Note que aqui ambos os termos dependem das fases os osciladores locais eletrônicos. Normalmente, podemos retirar todos estes osciladores locais de uma única fonte, o que faz com que cada $\theta_{i}$ esteja relacionado com cada $\theta_{j}$ por um valor fixo, $\theta_{i}=\theta_{j}+a_{i j}$. Desta forma, na presença de difusão de fase somente a componente não-estacionária irá se anular nas equações anteriores, como em (3.69). No caso particular em que $a_{i j}=0$, as componentes estacionárias se tornam

$$
\begin{aligned}
& \operatorname{Re}\left[S q_{i j}\right]=\frac{\left\langle\left(\delta \hat{i}_{\text {out }}^{c}\right)_{i}\left(\delta \hat{i}_{\text {out }}^{c}\right)_{j}\right\rangle+\left\langle\left(\delta \hat{i}_{\text {out }}^{s}\right)_{i}\left(\delta \hat{i}_{\text {out }}^{s}\right)_{j}\right\rangle}{2}, \\
& \operatorname{Im}\left[S q_{i j}\right]=\frac{\left\langle\left(\delta \hat{i}_{\text {out }}^{c}\right)_{i}\left(\delta \hat{i}_{\text {out }}^{s}\right)_{j}\right\rangle-\left\langle\left(\delta \hat{i}_{\text {out }}^{s}\right)_{i}\left(\delta \hat{i}_{\text {out }}^{c}\right)_{j}\right\rangle}{2},
\end{aligned}
$$

onde $S q_{i j}=\left\langle\delta \hat{q}_{i}\left(\Omega_{\mathrm{an}}\right) \delta \hat{q}_{j}\left(-\Omega_{\mathrm{an}}\right)\right\rangle$ são as componentes da matriz de covariâncias espectral. Isto faz com que cada componente da matriz espectral do campo refletido esteja relacionada com a do campo de entrada por

$$
\begin{aligned}
S q_{i j}^{R}\left(\Omega_{\mathrm{an}}\right)= & A_{x x}^{i j} C q_{i j}+A_{y y}^{i j} C p_{i j}+A_{x y}^{i j} C q p_{i j}+A_{y x}^{i j} C p q_{i j}+\delta_{i j}\left(\left|g_{x v}\right|^{2}+\left|g_{y v}\right|^{2}\right) \\
& 2 C q_{i j}\left(\Omega_{\mathrm{an}}\right)=\left\langle\hat{q}_{i+}^{i n} \hat{q}_{j+}^{i n}\right\rangle+\left\langle\hat{p}_{i-}^{i n} \hat{p}_{j-}^{i n}\right\rangle-i\left[\left\langle\hat{q}_{i+}^{i n} \hat{p}_{j-}^{i n}\right\rangle-\left\langle\hat{p}_{i-}^{i n} \hat{q}_{j+}^{i n}\right\rangle\right], \\
& 2 C p_{i j}\left(\Omega_{\mathrm{an}}\right)=\left\langle\hat{p}_{i+}^{i n} \hat{p}_{j+}^{i n}\right\rangle+\left\langle\hat{q}_{i-}^{i n} \hat{q}_{j-}^{i n}\right\rangle+i\left[\left\langle\hat{p}_{i+}^{i n} \hat{q}_{j-}^{i n}\right\rangle-\left\langle\hat{q}_{i-}^{i n} \hat{p}_{j+}^{i n}\right\rangle\right], \\
& 2 C q p_{i j}\left(\Omega_{\mathrm{an}}\right)=\left\langle\hat{q}_{i+}^{i n} \hat{p}_{j+}^{i n}\right\rangle_{S}-\left\langle\hat{q}_{i-}^{i n} \hat{p}_{j-}^{i n}\right\rangle_{S}+i\left[\left\langle\hat{q}_{i+}^{i n} \hat{q}_{j-}^{i n}\right\rangle+\left\langle\hat{p}_{i-}^{i n} \hat{p}_{j+}^{i n}\right\rangle\right], \\
& 2 C p q_{i j}\left(\Omega_{\mathrm{an}}\right)=\left\langle\hat{p}_{i+}^{i n} \hat{q}_{j+}^{i n}\right\rangle_{S}-\left\langle\hat{p}_{i-}^{i n} \hat{q}_{j-}^{i n}\right\rangle_{S}-i\left[\left\langle\hat{p}_{i+}^{i n} \hat{p}_{j-}^{i n}\right\rangle+\left\langle\hat{q}_{i-}^{i n} \hat{q}_{j+}^{i n}\right\rangle\right],
\end{aligned}
$$

onde $A_{k l}^{i j}\left(\Delta_{p}, \Omega^{\prime}\right)=g_{k}^{i}\left(\Delta_{p}^{i}, \Omega^{\prime}\right) g_{l}^{j}\left(\Delta_{p}^{j}, \Omega^{\prime}\right)^{*}$, com $k=x, y$ e $l=x, y$. O índice $i$ que está em $g^{i}$ representa as propriedades físicas de cada cavidade, como os coeficientes de reflexão e a distância entre os espelhos. Se todas as cavidades possuem propriedades físicas idênticas, o único parâmetro que de fato se modifica é a dessintonia de cada uma em relação à frequência da portadora, $g_{k}^{i}\left(\Delta_{p}^{i}, \Omega^{\prime}\right)=g_{k}\left(\Delta_{p}^{i}, \Omega^{\prime}\right)$. Se a fotocorrente for estacionária, $\left\langle\delta \hat{q}_{i}\left(\Omega_{\mathrm{an}}\right) \delta \hat{q}_{j}\left(\Omega_{\mathrm{an}}\right)\right\rangle=0$, fazendo com que haja vínculos entre as quantidades 
mostradas em 3.109-3.112,

$$
\begin{gathered}
\left\langle\hat{q}_{i+}^{i n} \hat{q}_{j+}^{i n}\right\rangle=\left\langle\hat{p}_{i-}^{i n} \hat{p}_{j-}^{i n}\right\rangle, \quad\left\langle\hat{p}_{i+}^{i n} \hat{p}_{j+}^{i n}\right\rangle=\left\langle\hat{q}_{i-}^{i n} \hat{q}_{j-}^{i n}\right\rangle, \quad\left\langle\hat{q}_{i+}^{i n} \hat{p}_{j+}^{i n}\right\rangle_{S}=-\left\langle\hat{q}_{i-}^{i n} \hat{p}_{j-}^{i n}\right\rangle_{S}, \quad\left\langle\hat{p}_{i+}^{i n} \hat{q}_{j+}^{i n}\right\rangle_{S}=-\left\langle\hat{p}_{i-}^{i n} \hat{q}_{j-}^{i n}\right\rangle_{S}, \\
\left\langle\hat{q}_{i+}^{i n} \hat{p}_{j-}^{i n}\right\rangle=-\left\langle\hat{p}_{i-}^{i n} \hat{q}_{j+}^{i n}\right\rangle, \quad\left\langle\hat{p}_{i+}^{i n} \hat{q}_{j-}^{i n}\right\rangle=-\left\langle\hat{q}_{i-}^{i n} \hat{p}_{j+}^{i n}\right\rangle, \quad\left\langle\hat{q}_{i+}^{i n} \hat{q}_{j-}^{i n}\right\rangle=\left\langle\hat{p}_{i-}^{i n} \hat{p}_{j+}^{i n}\right\rangle, \quad\left\langle\hat{p}_{i+}^{i n} \hat{p}_{j-}^{i n}\right\rangle=\left\langle\hat{q}_{i-}^{i n} \hat{q}_{j+}^{i n}\right\rangle .
\end{gathered}
$$

Isto faz com que a parte real de $S q_{i j}^{R}\left(\Omega_{\mathrm{an}}\right)$ seja igual à matriz de covariâncias dos modos em ambos os subespaços de soma e subtração (esta última rodada de $\pi / 2$ ). A parte imaginária de $S q_{i j}^{R}\left(\Omega_{\mathrm{an}}\right)$ se iguala às correlações entre estes dois subespaços.

A relação 3.108 é válida para toda a matriz de covariâncias espectral. Se $i=j$, obtemos a mesma relação mostrada em 3.97) para cada campo $i \operatorname{com} C q_{i i}=\alpha, C p_{i i}=\beta$, $C q p_{i i}=C p q_{i i}^{*}=\gamma+i \delta_{\mathrm{u}}$. Se $i \neq j$, as correlações de intensidade do campo refletido irão possuir partes real e imaginária que serão dadas por

$$
\operatorname{Re}\left[S q_{i j}^{R}\right]=\sum_{n=1}^{8} f_{n} X_{n} \quad \text { e } \quad \operatorname{Im}\left[S q_{i j}^{R}\right]=\sum_{n=1}^{8} f_{n}^{\prime} X_{n},
$$

onde as quantidades $f_{n}, f_{n}^{\prime}$ e $X_{n}$ são componentes dos seguintes vetores

$$
X=\left(\begin{array}{c}
\operatorname{Re}\left[C q_{i j}\right] \\
\operatorname{Re}\left[C p_{i j}\right] \\
\operatorname{Im}\left[C p_{i j}\right] \\
\operatorname{Im}\left[C p_{i j}\right] \\
\operatorname{Re}\left[C q p_{i j}\right] \\
\operatorname{Re}\left[C p q_{i j}\right] \\
\operatorname{Im}\left[C q p_{i j}\right] \\
\operatorname{Im}\left[C p q_{i j}\right]
\end{array}\right), \quad f=\left(\begin{array}{c}
\operatorname{Re}\left[A_{x x}^{i j}\right] \\
\operatorname{Re}\left[A_{y y}^{i j}\right] \\
-\operatorname{Im}\left[A_{x x}^{i j}\right] \\
-\operatorname{Im}\left[A_{y y}^{i j}\right] \\
\operatorname{Re}\left[A_{x y}^{i j}\right] \\
\operatorname{Re}\left[A_{y x}^{i j}\right] \\
-\operatorname{Im}\left[A_{x y}^{i j}\right] \\
-\operatorname{Im}\left[A_{y x}^{i j}\right]
\end{array}\right) \quad \text { e } \quad f^{\prime}=\left(\begin{array}{c}
\operatorname{Im}\left[A_{x x}^{i j}\right] \\
\operatorname{Im}\left[A_{y y}^{i j}\right] \\
\operatorname{Re}\left[A_{x x}^{i j}\right] \\
\operatorname{Re}\left[A_{y y}^{i j}\right] \\
\operatorname{Im}\left[A_{x y}^{i j}\right] \\
\operatorname{Im}\left[A_{y x}^{i j}\right] \\
\operatorname{Re}\left[A_{x y}^{i j}\right] \\
\left.\operatorname{Re}\left[A_{y x}^{i j}\right]\right)^{T}
\end{array}\right)
$$

Note que todos os segundos momentos envolvendo quantidades pertencentes aos dois campos estão presentes do vetor $X$ e que $f$ e $f^{\prime}$ possuem as mesmas componentes a menos do sinal de algumas delas e do ordenamento. No caso em que todas as componentes de $f$ são não-nulas e independentes, apenas a parte real ou imaginária da fotocorrente é necessária para inferir todas as propriedades do estado. A medida da parte imaginária fornece informação redundante que pode ser utilizada para reduzir as incertezas das componentes do vetor $X$ após os procedimentos de inferência. 


\subsubsection{Relações entre Operadores Espectrais e das Bandas Laterais}

Como discutido no decorrer do capítulo, a fotodetecção no domínio da frequência privilegia os modos de soma e subtração das bandas laterais. A medida direta da fotocorrente após a demodulação nos permite acessar os modos $\hat{q}_{i+}\left(\theta_{i}\right)$ e $\hat{p}_{-}\left(\theta_{i}\right)$ utilizando dois osciladores locais em quadratura. Ambos operadores são hermitianos e podem ser interpretados como observáveis físicos. Ambos podem ser combinados para formar o operador de quadratura espectral $\delta \hat{q}(\Omega)$, como mostrado em $(3.14)$, que equivale à transformada de Fourier. Este operador é não-hermitiano e, consequentemente, não pode ser associando a um observável físico de um modo do campo. Entretanto ele é importante justamente pelo fato de se tratar do operador obtido após a transformada de Fourier de um operador temporal. Ele também possui um papel importante quando tratamos de sistemas estacionários, como discutido no decorrer deste capítulo.

Por este motivo, é importante ter uma forma simples e operacional de se converter os operadores espectrais para os operadores hermitianos que representam cada modo do campo. Para isto, reescrevemos a expressão (3.14) como

$$
\begin{array}{r}
\delta \hat{q}( \pm \Omega)=\frac{\hat{q}_{+} \pm i \hat{p}_{-}}{\sqrt{2}}=\frac{1}{2}\left[\hat{q}_{+\Omega}+\hat{q}_{-\Omega} \pm i \hat{p}_{+\Omega} \mp i \hat{p}_{-\Omega}\right] \\
\text { e } \delta \hat{p}( \pm \Omega)=\frac{\hat{p}_{+} \mp i \hat{q}_{-}}{\sqrt{2}}=\frac{1}{2}\left[\hat{p}_{+\Omega}+\hat{p}_{-\Omega} \mp i \hat{q}_{+\Omega} \pm i \hat{q}_{-\Omega}\right]
\end{array}
$$

onde $\delta \hat{q}( \pm \Omega) \equiv \delta \hat{q}_{0}( \pm \Omega)$ e $\delta \hat{p}( \pm \Omega) \equiv \delta \hat{q}_{\pi / 2}( \pm \Omega)$. Para apenas um campo, estas relações podem ser colocadas na forma vetorial como

$$
\begin{gathered}
\hat{\xi}(\Omega)=L_{ \pm} \hat{\xi}_{\Omega}^{ \pm}=L \hat{\xi}_{\Omega}, \quad \text { com } \hat{\xi}(\Omega)=(\delta \hat{q}(+\Omega), \delta \hat{p}(+\Omega), \delta \hat{q}(-\Omega), \delta \hat{p}(-\Omega))^{T} \\
\hat{\xi}_{\Omega}^{ \pm}=\left(\hat{q}_{+}, \hat{p}_{+}, \hat{q}_{-}, \hat{p}_{-}\right)^{T}, \quad \hat{\xi}_{\Omega}=\left(\hat{q}_{+\Omega}, \hat{p}_{+\Omega}, \hat{q}_{-\Omega}, \hat{p}_{-\Omega}\right)^{T} \\
L_{ \pm}=\frac{1}{\sqrt{2}}\left(\begin{array}{cc}
\mathcal{I} & i \mathcal{J} \\
\mathcal{I} & -i \mathcal{J}
\end{array}\right) \text { e } L=\frac{1}{2}\left(\begin{array}{cc}
\mathcal{I}+i \mathcal{J} & \mathcal{I}-i \mathcal{J} \\
\mathcal{I}-i \mathcal{J} & \mathcal{I}+i \mathcal{J}
\end{array}\right), \text { onde } \mathcal{J}=\left(\begin{array}{cc}
0 & 1 \\
-1 & 0
\end{array}\right)
\end{gathered}
$$

e $\mathcal{I}=\operatorname{diag}(1,1)$ é a matriz identidade em duas dimensões. As matrizes de transformação tem as propriedades $L_{ \pm}^{-1}=L_{ \pm}^{+}$e $L=L^{-1}=L^{\dagger}$. Para um sistema composto por $n$ campos é conveniente adotar a ordenação $\hat{\xi}_{\Omega}^{ \pm}=\left(\hat{q}_{1+}, \hat{p}_{1+}, \ldots, \hat{q}_{n+}, \hat{p}_{n+}, \hat{q}_{1-}, \hat{p}_{1-}, \ldots, \hat{q}_{n-}, \hat{p}_{n-}\right)^{T}$ e formas similares para $\hat{\xi}_{\Omega}$ e $\hat{\xi}(\Omega)$. Nesta ordenação, as matrizes de transformação terão a mesma forma que em (3.118), desde que as matrizes $\mathcal{J}$ e $\mathcal{I}$ sejam da forma

$$
\mathcal{J}=\left(\begin{array}{cc}
0 & I \\
-I & 0
\end{array}\right) \quad \text { e } \quad \mathcal{I}=\left(\begin{array}{ll}
I & 0 \\
0 & I
\end{array}\right)
$$


onde $I$ é a matriz identidade de dimensão $n$.

A matriz de covariâncias espectral completa, $V_{c}(\Omega)$, é definida por

$$
V_{c}(\Omega)=\left\langle\xi(\Omega)[\xi(\Omega)]^{\dagger}\right\rangle=\left(\begin{array}{cc}
S(\Omega) & S^{n e}(-\Omega) \\
S^{n e}(\Omega) & S(-\Omega)
\end{array}\right)
$$

onde $S(\Omega)$ é a matriz de covariâncias espectral e a matriz $S^{\text {ne }}(\Omega)$ é formada pelos termos que se anulam para uma fotocorrente estacionária, como $\langle\hat{q}(\Omega) \hat{q}(\Omega)\rangle$. Portanto, as matrizes de covariâncias dos operadores de quadratura nas bandas laterais estão relacionadas a $V_{c}(\Omega)$ por

$$
V_{c}(\Omega)=L_{ \pm} V_{\Omega}^{ \pm} L_{ \pm}^{\dagger}=L V_{\Omega} L^{\dagger}
$$

Se a fotocorrente for estacionária, $S^{n e}(\Omega)_{i j}=0$. Nestas condições, $V_{c}(\Omega)=\operatorname{diag}\left(S(\Omega), S(\Omega)^{*}\right)^{T}$ e a matriz $V_{\Omega}^{ \pm}$se torna

$$
V_{\Omega}^{ \pm}=\left(\begin{array}{cc}
\operatorname{Re}[S(\Omega)] & \operatorname{Im}[S(\Omega)] \mathcal{J} \\
-\operatorname{Im}[S(\Omega)] \mathcal{J} & \mathcal{J} \operatorname{Re}[S(\Omega)] \mathcal{J}
\end{array}\right)
$$

Portanto, a parte real da matriz espectral pode ser interpretada como uma matriz de covariâncias de operadores no espaço de fase quando a fotocorrente é estacionária. De acordo com (3.122, ela é a matriz de covariâncias dos operadores no subespaço de soma das bandas laterais. Note que a matriz $\mathcal{J}$ representa uma rotação de $\pi / 2$ no espaço de quadraturas. Quando aplicada somente no subespaço de subtração, o resultado é uma matriz de covariâncias na forma

$$
V_{\Omega}^{ \pm \prime}=\left(\begin{array}{cc}
\operatorname{Re}[S(\Omega)] & \operatorname{Im}[S(\Omega)] \\
-\operatorname{Im}[S(\Omega)] & \operatorname{Re}[S(\Omega)]
\end{array}\right)
$$

para a ordenação $\hat{\xi}_{\Omega}^{ \pm \prime}=\left(\hat{q}_{1+}, \hat{p}_{1+}, \ldots, \hat{q}_{n+}, \hat{p}_{n+}, \hat{p}_{1-},-\hat{q}_{1-}, \ldots, \hat{p}_{n-},-\hat{q}_{n-}\right)^{T}$. Desta forma, a parte real da matriz de covariâncias espectral, $S(\Omega)$, possui sentido físico, pois se iguala à matriz de covariâncias dos modos de soma das bandas laterais. Se $\operatorname{Im}[S(\Omega)]=0$, a descrição dada por $V_{ \pm \Omega}$ ou por $S(\Omega)$ são equivalentes. Se $\operatorname{Im}[S(\Omega)] \neq 0, \operatorname{Re}[S(\Omega)]$ representa a matriz de covariâncias do sistema reduzido obtido a partir do traço parcial nos modos de subtração de bandas laterais.

No próximo capítulo apresentarei o aparato experimental que nos permite tanto preparar os estados quânticos do campo eletromagnético quanto realizar a fotodetecção espectral discutida no decorrer deste capítulo. Nele procurei dar um panorama geral do experimento e de como as partes do aparato experimental são combinadas, seguido 
de uma explicação detalhada do papel que cada uma das partes desempenha. 


\section{Descrição do Aparato Experimental}

O nosso aparato experimental consiste basicamente de dois estágios. O primeiro deles é a preparação de estados quânticos codificados nas flutuações das quadraturas fase e amplitude dos 3 campos de luz gerados pelo OPO - um com comprimento de onda em $532 \mathrm{~nm}$ e dois por volta de $1064 \mathrm{~nm}$. Os componentes desta etapa são o campo de bombeio, o OPO, a cavidade de filtro e os sistemas de estabilização de temperatura e frequência. O segundo estágio consiste na detecção e aquisição das flutuações das quadraturas e é formada pelas cavidades de análise, pela cadeia de demodulação e pelos conversores analógico-digital. Descreverei cada uma destas etapas a seguir.

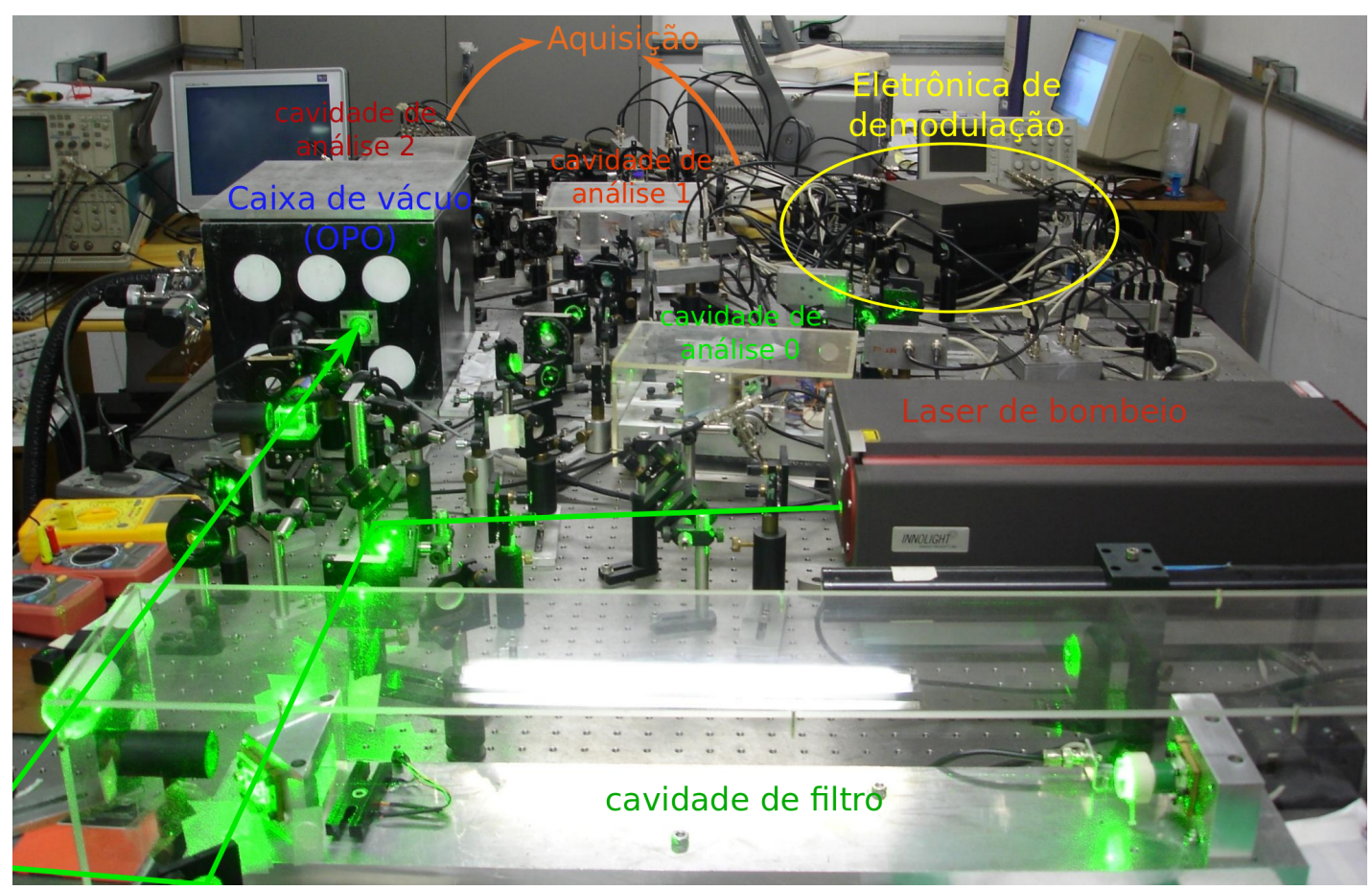

Figura 4.1: Foto da mesa ótica onde é possivel ver todos os elementos menos a eletrônica de controle que se encontra embaixo da mesa. O OPO está à esquerda dentro da câmara de vácuo em forma de dado. A seta verde indica o caminho do bombeio até chegar ao OPO. 


\subsection{Laser de Bombeio, OPO e Cavidade de Filtro}

Utilizamos um cristal não-linear de tinanil-fosfato de potássio $(K T P)-\mathrm{KTiOPO}_{4}$ do tipo II, que possui $12 \mathrm{~mm}$ de comprimento, para gerar a conversão paramétrica [90, 91, 92]. O bombeio consiste de um laser de Nd:YAG dobrado, modelo Diabolo, produzido pela Innolight, com comprimento de onda de $532 \mathrm{~nm}$ (verde), largura de linha de $1 \mathrm{kHz}{ }^{1}$ e com mais de $1 \mathrm{~km}$ de comprimento de coerência. Todo o seu sistema de controle e estabilização de frequência é fornecido pelo fabricante. Os feixes gêmeos

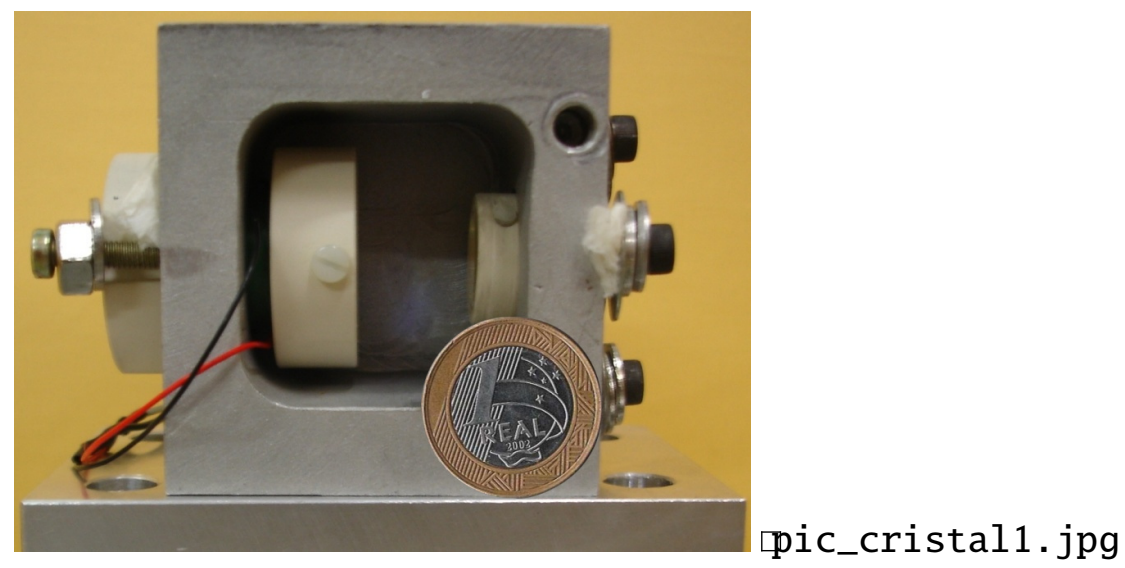

Figura 4.2: A direta está uma foto do bloco de aluminio onde está montada a cavidade ótica e a esquerda uma foto do cristal de KTP é mostrada.

possuem frequências distintas, mas bastante próximas de modo que os comprimentos de onda estão em torno de $1064 \mathrm{~nm}$ (infravermelho próximo) $)^{2}$. A cavidade do OPO é do tipo linear e é montada em um bloco rígido de alumínio. A cavidade é ressonante tanto para os feixes gêmeos quanto para o bombeio. Os resultados mostrados aqui foram obtidos com dois OPOs. A montagem mecânica da cavidade também foi ligeiramente modificada. A primeira é mostrada na figura $4.2 \mathrm{e}$ a segunda na figura 4.3 .

- OPO 1:

O espelho de entrada/saída do bombeio possui coeficiente de reflexão $R_{v}=70 \%$ para o verde e de mais de $99 \%$ para o infravermelho. O espelho de acoplamento para os feixes gêmeos possui reflexão de $R_{I R}=96 \%$ para os mesmos e de mais de $99 \%$ para o verde. Ambos os espelhos possuem um raio de curvatura de 5 $\mathrm{cm}$. Como a distância entre os espelhos é de $19 \mathrm{~mm}$ e o índice de refração do

\footnotetext{
${ }^{1}$ Esta largura de linha é medida no intervalo de $100 \mathrm{~ms}$, porém ainda há um drift térmico de $2 \mathrm{MHz} / \mathrm{min}$ que alarga a linha do laser para tempos longos.

${ }^{2}$ As frequências dos feixes gêmeos podem ser ajustadas modificando-se a temperatura do cristal. Nós usamos este grau de liberdade para otimizar a potência dos feixes gêmeos.
} 


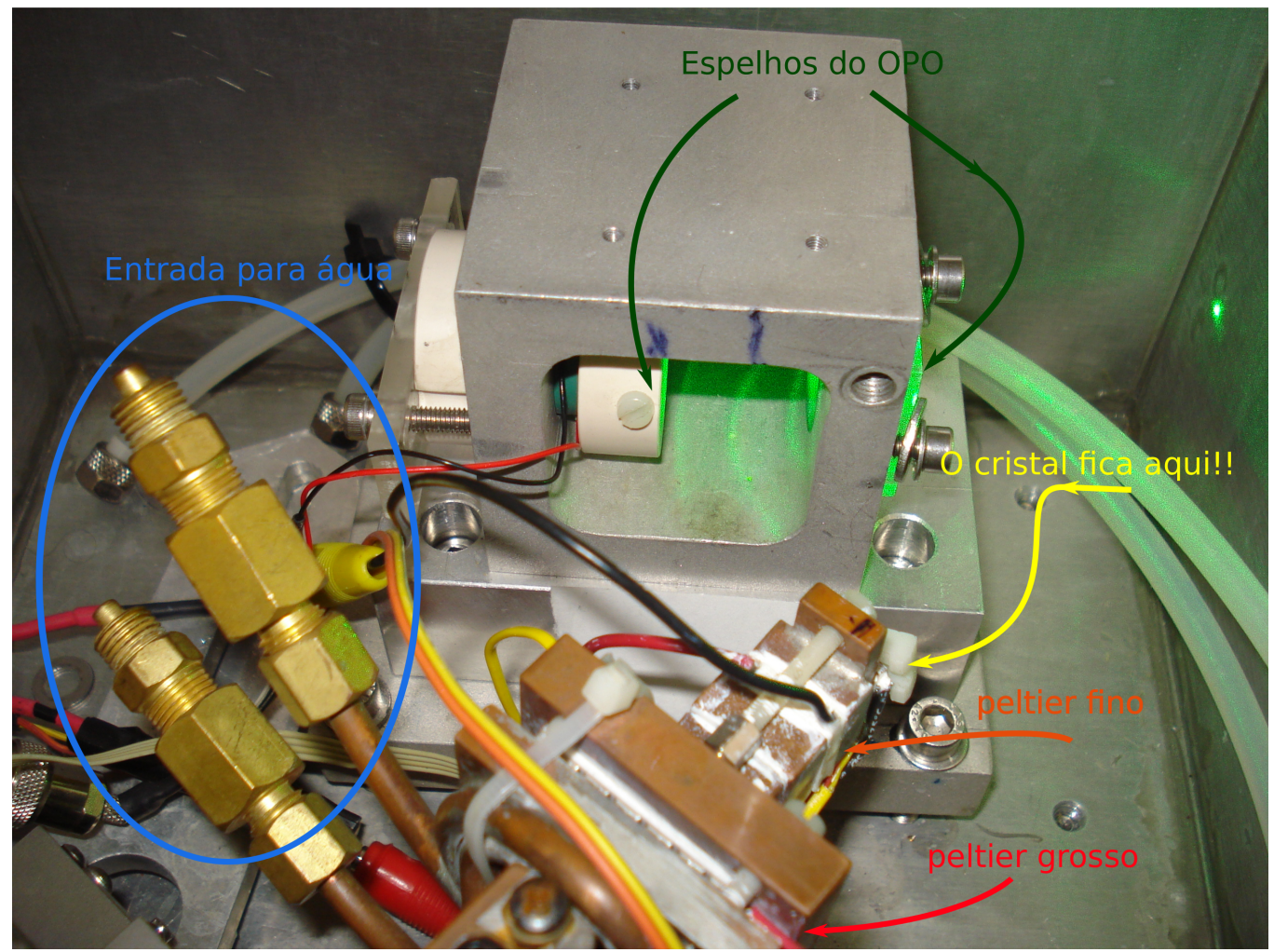

Figura 4.3: Foto do interior da caixa de vácuo mostrando a cavidade formada pelos dois espelhos fixados em um bloco de aluminio. A peça ao centro é usada como base para o cristal, que fica na ponta da mesma. Dois elementos peltier controlam a temperatura do cristal, o que permite estabilizar a mesma com uma precisão da ordem de $m \mathrm{~K}$ e fazer medidas desde $-20 \mathrm{C}^{\circ}$ até $60 \mathrm{C}^{\circ}$. As estruturas de cobre servem para garantir o contato térmico entre o peltier e o cristal. Parte do resfriamento é feito de maneira externa por meio de uma geladeira que bombeia água fria para dentro da câmara de vácuo.

cristal de KTP é de aproximadamente 1.8, o FSR é de $\Delta f_{v}=\Delta f_{I R}=5.24(0.16) \mathrm{GHz}$ [56]. As finesses são de aproximadamente $F_{I R}=120(5)$ para os feixes gêmeos e de $F_{v}=18(1)$ para o verde 3 , o que faz com que as larguras de banda sejam de $\delta f_{v}=290(25) \mathrm{MHz}$ para o bombeio e de $\delta f_{I R}=44(3) \mathrm{MHz}$ para os feixes gêmeos. O limiar de oscilação para este $O P O$ varia entre $55 \mathrm{~mW}$ e $70 \mathrm{~mW}$ de acordo o grau de sujeira dos espelhos do OPO e da cavidade de filtro. Como nosso cristal é do Tipo II, separamos os feixes gêmeos usando de um divisor de feixes polarizante e cada um é enviado para seu respectivo sistema de detecção.

\section{- OPO 2:}

O espelho de entrada/saída do bombeio possui coeficiente de reflexão $R_{v}=89 \%$ para o verde e de mais de $99 \%$ para o infravermelho. O espelho de acoplamento para os feixes gêmeos possui reflexão de $R_{I R}=95 \%$ para os mesmos e de mais

\footnotetext{
${ }^{3}$ As finesses podem mudar ligeiramente de um dia para o outro. A precisão mostrada para estes valores reflete somente o erro do osciloscópio utilizado para medi-la.
} 
de 99\% para o verde. Ambos espelhos possuem raio de curvatura de $2.5 \mathrm{~cm}$, que é mais próxima da condição ótima de operação que a cavidade do OPO 1 [93]. Como o tamanho da cavidade aumentou para 54(2) mm, o FSR é de $\Delta f_{v}=\Delta f_{I R}=4.10(0.15) \mathrm{GHz}$. As finesses são de $F_{v}=41(2)$ para o bombeio e $F_{I R}=100(4)$. Consequentemente, as larguras de banda são $\delta f_{v}=100(9) \mathrm{MHz}$ e $\delta f_{I R}=41(3) \mathrm{MHz}$ para o bombeio e feixes gêmeos, respectivamente. Esta cavidade foi montada com o objetivo específico que estudar o campo de bombeio.

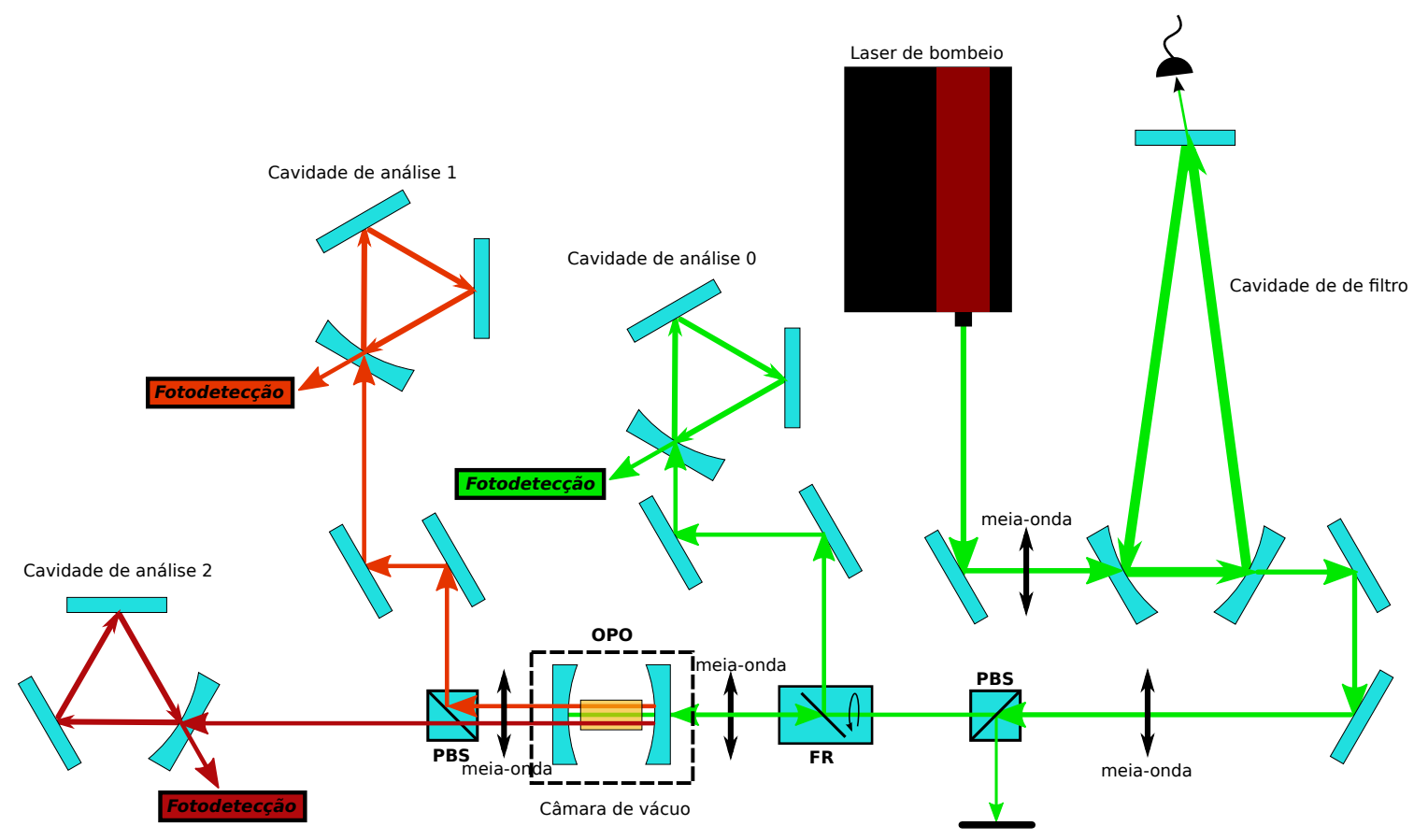

Figura 4.4: Figura esquemática do experimento. Nela podemos ver todos os elementos importantes mostrados na foto 4.1. Escrevo o nome fotodetecção após as cavidades de análise, pois a forma como fazemos isso foi continuamente aperfeiçoada e será descrita com mais detalhes adiante.

Para garantir que o bombeio seja limitado a um estado coerente, há uma cavidade vazia antes do OPO, que vai filtrar, quando em ressonância, frequências fora da sua largura de banda. Ela tem por volta de $1 \mathrm{~m}$ de comprimento, Finesse por volta de $F_{\text {filtro }} \approx$ 350 e, consequentemente, largura de banda de aproximadamente $\delta f_{\text {filtro }} \approx 850 \mathrm{kHz}$. O campo transmitido por esta cavidade é utilizado para bombear o OPO. Entre a cavidade de filtro e o OPO há duas lâminas de meia-onda, um divisor de feixes polarizante $(P B S)$ e um rotator de Faraday (FR). A primeira lâmina é usada em conjunto com o PBS como um atenuador controlável. A segunda lâmina é utilizada para otimizar a polarização do bombeio, necessária para o processo de conversão paramétrica [56]. O rotator de Faraday é usado em conjunto com o PBS para separar o campo incidente do campo 
refletido pela cavidade e enviar este último para a cavidade de análise.

\subsection{Detetores e Filtros Eletrônicos}

As medidas de flutuações de intensidade são feitas de forma direta, pois nossos detetores são rápidos o suficiente para capturar pequenas mudanças no valor da intensidade. Como explicado no capítulo 3, utilizamos fotodiodos para converter o feixe de luz em uma fotocorrente. Especificamente, usamos os fotodiodos Epitaxx ETX300, que possui eficiência quântica de aproximadamente $95 \%$, para os feixes gêmeos e Hamamatsu S5973-02, com eficiência quântica de 94\%, para o bombeiq 4 As larguras de banda dos fotodiodos são de $1 \mathrm{GHz}$ e $500 \mathrm{MHz} 2^{5}$ para os modelos Epitaxx e Hamamatsu. Eles são montados em um circuito que conta com um amplificador de transimpedância 6 , um divisor de tensão sensível à frequência, que consiste de um filtro passa alta em uma das saídas e um passa baixa na outra 7 e diversos amplificadores. A frequência de corte usada para separar altas e baixas frequências é de $300 \mathrm{kHz}$ em nosso experimento. Sinais que oscilam com frequências menores que este valor são chamados de DC (Direct Current) e acima do mesmo, de HF (High Frequency) 8 . Como a largura de linha do laser é bem menor que $300 \mathrm{kHz}$, os sinais HF já possuem média nula.

Se medíssemos o sinal HF diretamente na saída do detetor, iríamos ter todas as componentes de frequência, acima dos $300 \mathrm{kHz}$ e abaixo da largura de banda do fotodiodo. Entretanto antes da aquisição existem ainda duas etapas de eletrônica. A primeira delas consiste de um filtro rejeita-faixa, centrado em duas frequências, 12 $\mathrm{MHz}$ e $24 \mathrm{MHz}$, que são bastante intensas, pois correspondem à frequência utilizada no sistema de travamento do Diabolo e a seu respectivo segundo harmônico. A outra etapa é a demodulação que faz a conversão do sinal HF centrado na frequência de análise para o sinal DC, permitindo que este seja capturado pelas placas de aquisição. Esta é realizada por um conversor analógico-digital da National Instruments e ocorre à taxa máxima de $5 \mathrm{MHz}$.

\footnotetext{
${ }^{4}$ Nós reinjetamos parte da luz refletida pela janela de proteção do fotodiodo.

${ }^{5}$ Dados do fabricante.

${ }^{6}$ Estes são circuitos que convertem sinal de corrente em tensão.

${ }^{7}$ Filtros passa alta (baixa) são circuitos que possuem uma resistência dependente da frequência, que se torna muito baixa para altas (baixas) frequências [94].

${ }^{8} \mathrm{Na}$ prática, a eletrônica atenua as frequências acima de $300 \mathrm{kHz}$ em uma das saídas e atenua as menores que $300 \mathrm{kHz}$ na outra.
} 


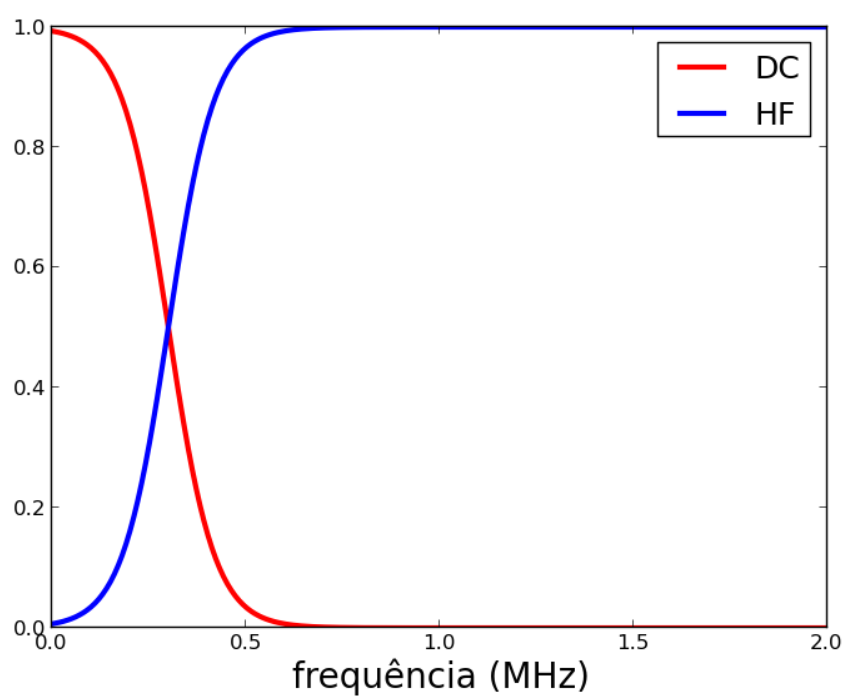

Figura 4.5: Filtragem que ocorre no fotodetector. A saída HF contém as componentes de alta frequência, enquanto na saída DC está as componentes de baixa frequência, incluindo os valores médios dos campos.

\subsection{Cadeia de Demodulação e Aquisição}

Para operação ótima, a taxa de aquisição, $\tau$, deve ser igual ao tempo de integração da demodulação. Este tempo é da ordem do inverso da largura de banda do filtro passa-baixa ao final do circuito de demodulação - esta é considerada nula em (3.44). Se escolhemos $\tau$ menor que o tempo de integração teremos informação redundante e com isto estaríamos perdendo precisão. Por exemplo, se este fosse igual à metade do tempo de integração, cada duas aquisições seriam equivalentes a um período de integração (ou filtragem) da demodulação e metade dos nossos pontos seria redundante. Este caso representa mais um desperdício do que um problema em si, visto que não há perda de informação. Uma situação mais crítica ocorre se $\tau$ for maior que o tempo de integração. Neste caso, deixamos de adquirir metade dos eventos e, consequentemente, estaríamos perdendo informação. No nosso experimento, a largura de banda da demodulação também foi escolhida como $600 \mathrm{kHz}$. Isto faz com que $\tau \approx 1.67 \mu$ s no nosso experimento. Este valor deve estar muito abaixo do tempo de difusão de fase do laser de bombeio - que, segundo o fabricante, é da ordem de $1 \mathrm{~ms}$. O número de pontos adquiridos é escolhido de acordo com a estabilidade do sistema experimental. Em geral fazemos aquisições de 450 mil e 600 mil pontos - o que corresponde a escolher um tempo total de aquisição de $T \approx 0.75 \mathrm{~s}$ e $T \approx 1 \mathrm{~s}$, respectivamente. Um exemplo pictórico da aquisição para um conjunto de 20 pontos é mostrado na figura 4.6 e um exemplo real retirado de uma das nossas medidas é mostrado em 4.7 


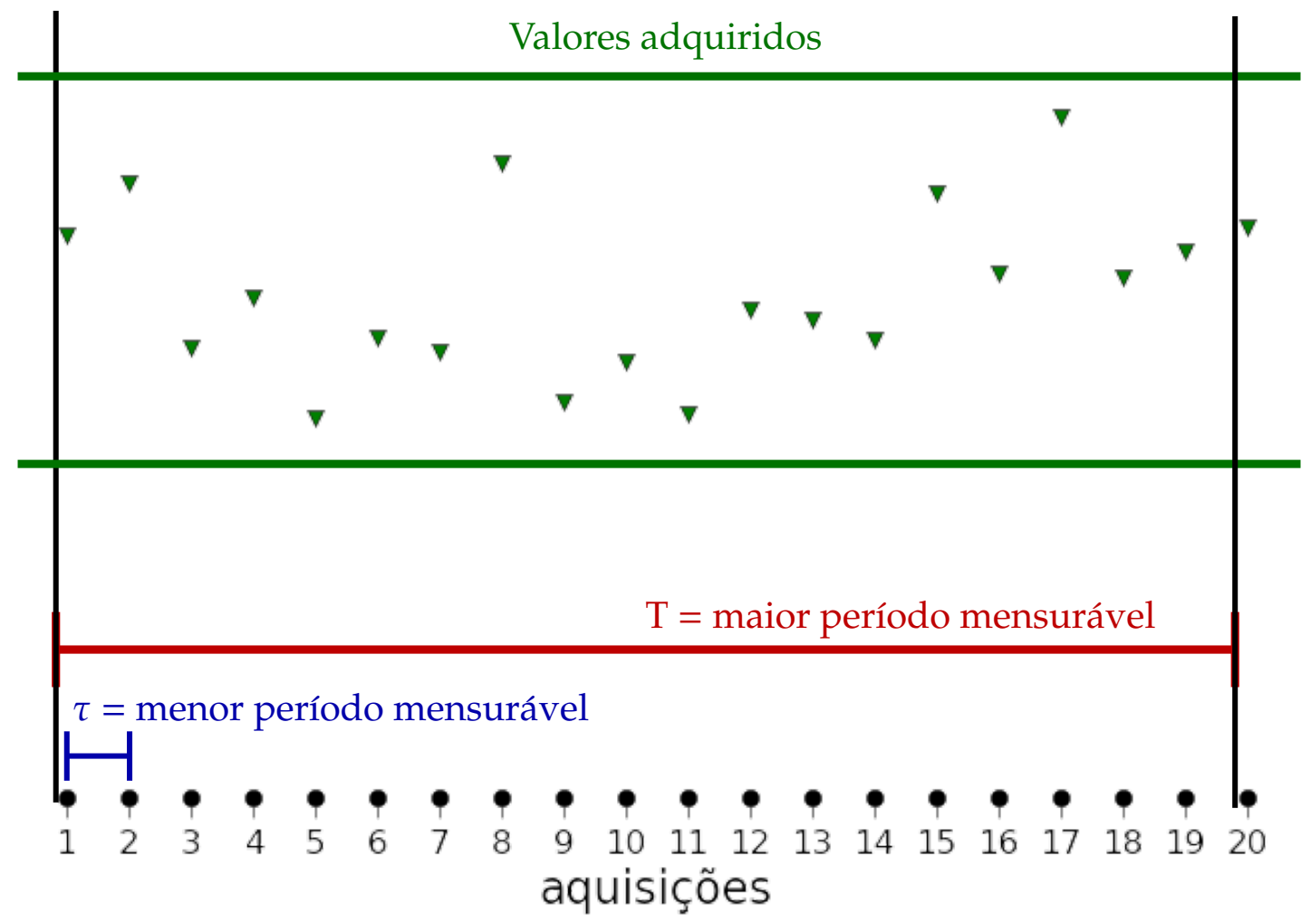

Figura 4.6: Figura esquemática mostrando o processo de aquisição e suas limitações para um conjunto de 20 aquisições Os períodos maior e menor que podem ser medidos são iguais, respectivamente, ao tempo de aquisição e à taxa de aquisição ('sampling'). Para cada aquisição, o valor do parâmetro físico é registrado, como mostrado nos triângulos verdes.

Como cada aquisição é uma realização diferente do experimento, usamos os valores medidos para calcular as propriedades estatísticas de interesse. As mais importantes para a nossa análise do emaranhamento são as variâncias e covariâncias em função das dessintonias das cavidades de análise. Estas são calculadas selecionando-se tipicamente conjuntos de 1000 pontos, pois este é um número grande o suficiente para nos permitir fazer uma estatística confiável, mas que também é pequeno comparado com os 450 mil pontos adquiridos. Neste caso, podemos ter curvas após o cálculo do ruído que podem ser comparadas com o modelo para a cavidade como discutido no capítulo 3. 

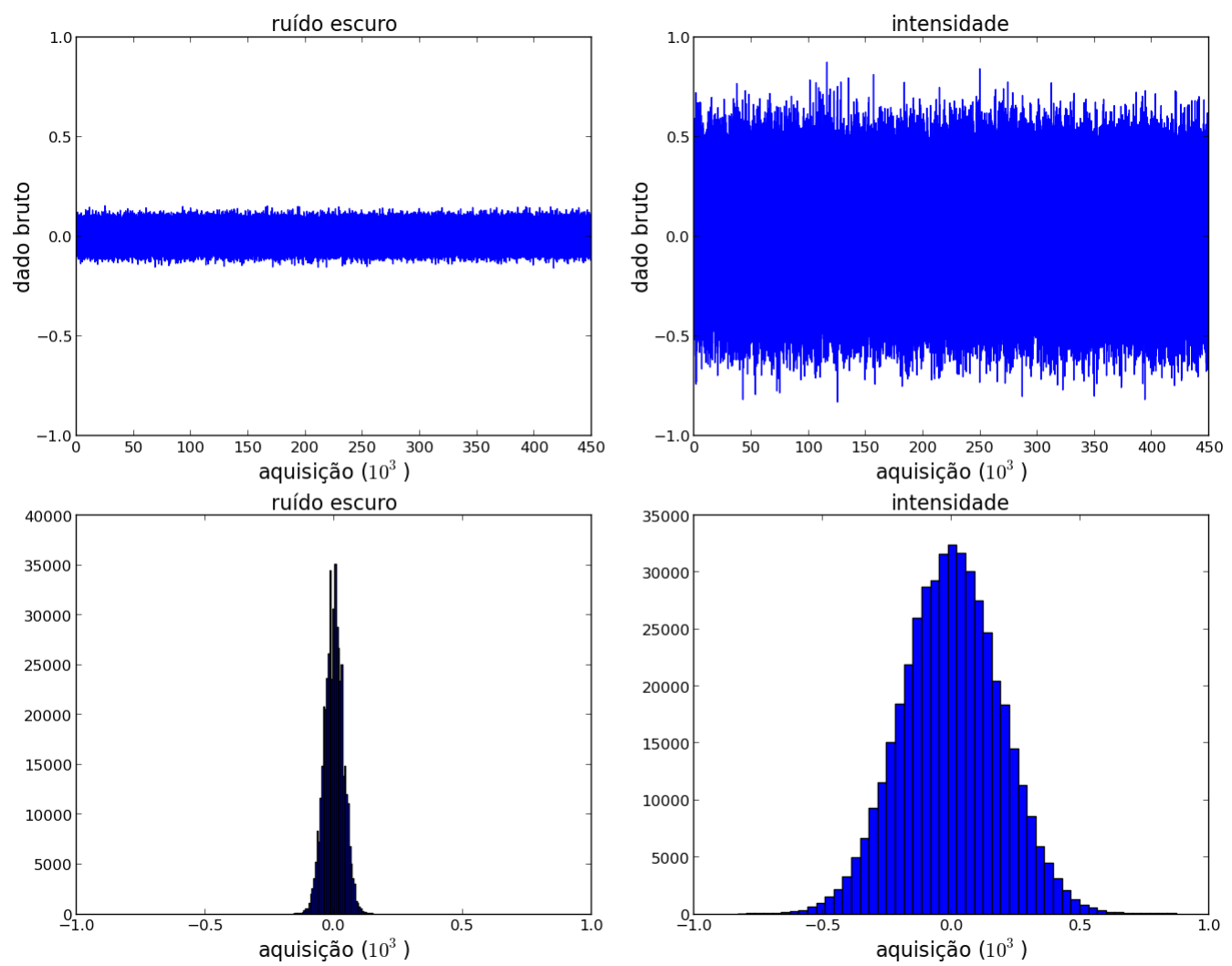

Figura 4.7: Figuras comparando os sinais adquiridos por um dos canais com e sem luz incidente: nas curvas da esquerda não há luz e estamos medindo o ruído escuro, nas curvas da direita é mostrado um dos canais dos feixes gêmeos. A cavidade de análise foi mantida fora de ressonância nesta aquisição, portanto, estamos observando somente intensidade nesta medida específica. As curvas inferiores são um histograma das superiores. Note que há uma forte indicação da gaussianidade do sistema. Uma análise mais detalhada deste problema será realizada no capítulo ??.

\subsection{Calibração do Shot Noise}

Utilizamos três métodos de análise da fotodetecção durante meu doutorado. Os dois primeiros são mostradas da figura 4.8 e serão discutidas em seguida. A terceira forma é uma variante da mostrada na figura $4.8 \mathrm{~b}$ ), na qual dividimos o sinal HF de cada detector e fazemos a demodulação de cada um deles com dois osciladores locais eletrônicos em quadratura, como discutido no capítulo 3. Este método de fotodetecção será discutido em mais detalhes no capítulo 7/A mudança foi feita para garantir uma melhor calibração do shot noise. No sistema mostrado na figura 4.8a) cada campo gerado pelo OPO era enviado a dois detectores, cujos sinais eram somados eletronicamente. Nesta configuração, não é possível fazer a calibração do shot noise para cada aquisição de dados, pois não temos acesso à subtração das fotocorrentes de cada detector. Desta 


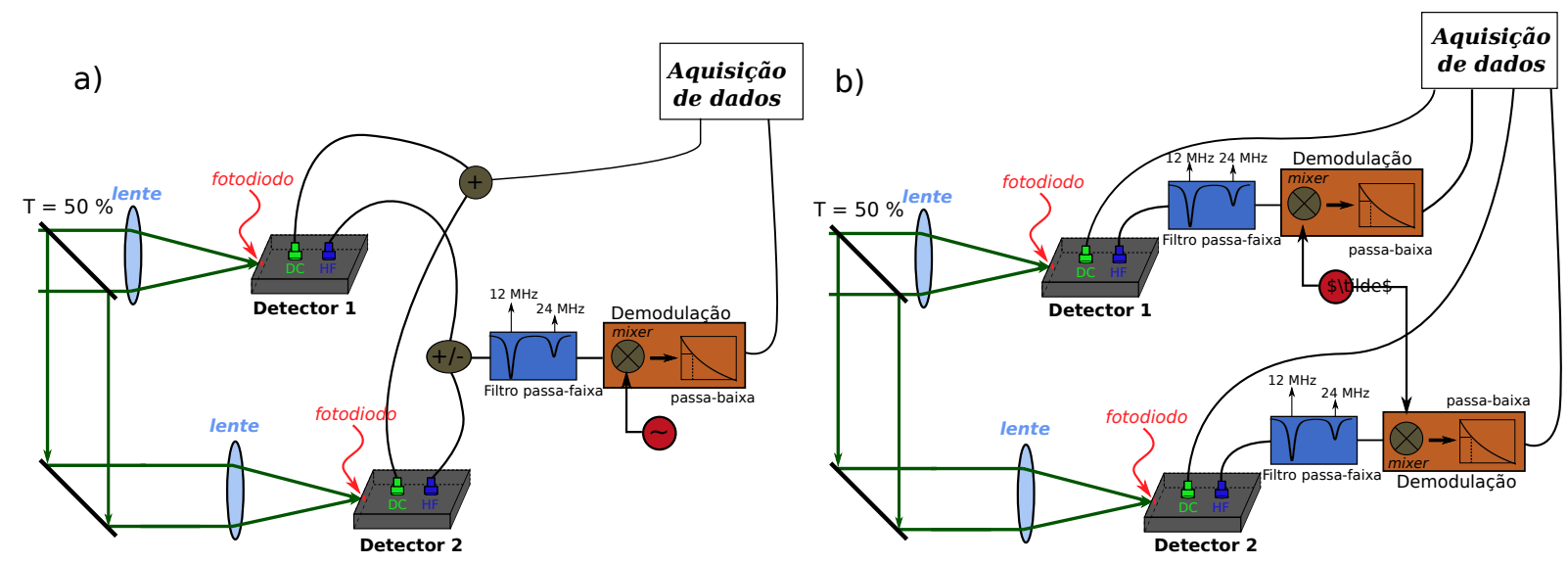

Figura 4.8: Comparação entre dois dos sistemas de detecção usados. Ambos realizam a demodulação com apenas uma componente do oscilador local eletrônico. A vantagem da medida b), consiste no fato de que ambas as fotocorrentes são registradas. Isto permite que com que tanto a soma quando a subtração dos sinais HF sejam calculados para cada aquisição.

forma, era necessário que antes e depois de cada bateria de medida fossem realizadas medidas complementares de calibração a partir das quais o coeficiente angular do shot noise era extraído, como mostrado na figura 3.10. A medidas de flutuação eram, por sua vez, comparadas com esta referência pré-calibrada. Este tipo de procedimento leva a uma diminuição na precisão com que resolvemos o shot noise, o que faz com que tenhamos uma menor precisão nas medidas de flutuação de intensidade. Para obter o sinal de subtração, usávamos o sub-harmônico do bombeio, fornecido pelo próprio Diabolo, o qual era alinhado sobre os feixes gêmeos. O PBS logo após o OPO era utilizado para dividir a potência do laser e assim, medir o sinal de subtração. Para o bombeio, usávamos uma chave para alternar o circuito que somava os sinais HF dos detetores como mostrado na figura 4.8 a).

No sistema de detecção mostrado da figura $4.8 \mathrm{p}$ ), os sinais HF de ambos detetores são adquiridos de maneira independente. Isto permite que o shot noise seja calibrado para cada medida. Como mostrado em (3.21) (3.16), os operadores que representam as saídas HF exatamente após os detetores 1 e 2 são

$$
\delta I_{1}(t)=\frac{1}{2} \sqrt{K}|g| \sqrt{\bar{I}}\left[\delta q(t)+\delta q_{v a c}(t)\right] \quad \text { e } \quad \delta I_{2}(t)=\frac{1}{2} \sqrt{K}|g| \sqrt{\bar{I}}\left[\delta q(t)-\delta q_{v a c}(t)\right]
$$

já que os valores médios são filtrados e vão para a saída DC. O operador $\delta q_{v a c}(t)$ representa o estado de vácuo que entra pela porta vazia dos divisores de feixes mostrados na figura 4.8. Como as equações (4.1) são lineares, sua forma é mantida quando passamos para o domínio da frequência e, pelo fato de $\delta q_{v a c}(\Omega)$ representar o estado de 
vácuo, $\left\langle\delta q_{v a c}(-\Omega) \delta q_{v a c}(\Omega)\right\rangle=1$. A flutuação de quadratura no domínio da frequência e já calibrada pelo shot noise então se torna

$$
\delta q(\Omega)=\frac{\delta I_{+}(\Omega)}{\left\langle\delta I_{-}(-\Omega) \delta I_{-}(\Omega)\right\rangle}, \quad \text { onde } \quad \delta I_{ \pm}=\delta I_{1}-\delta I_{2}
$$
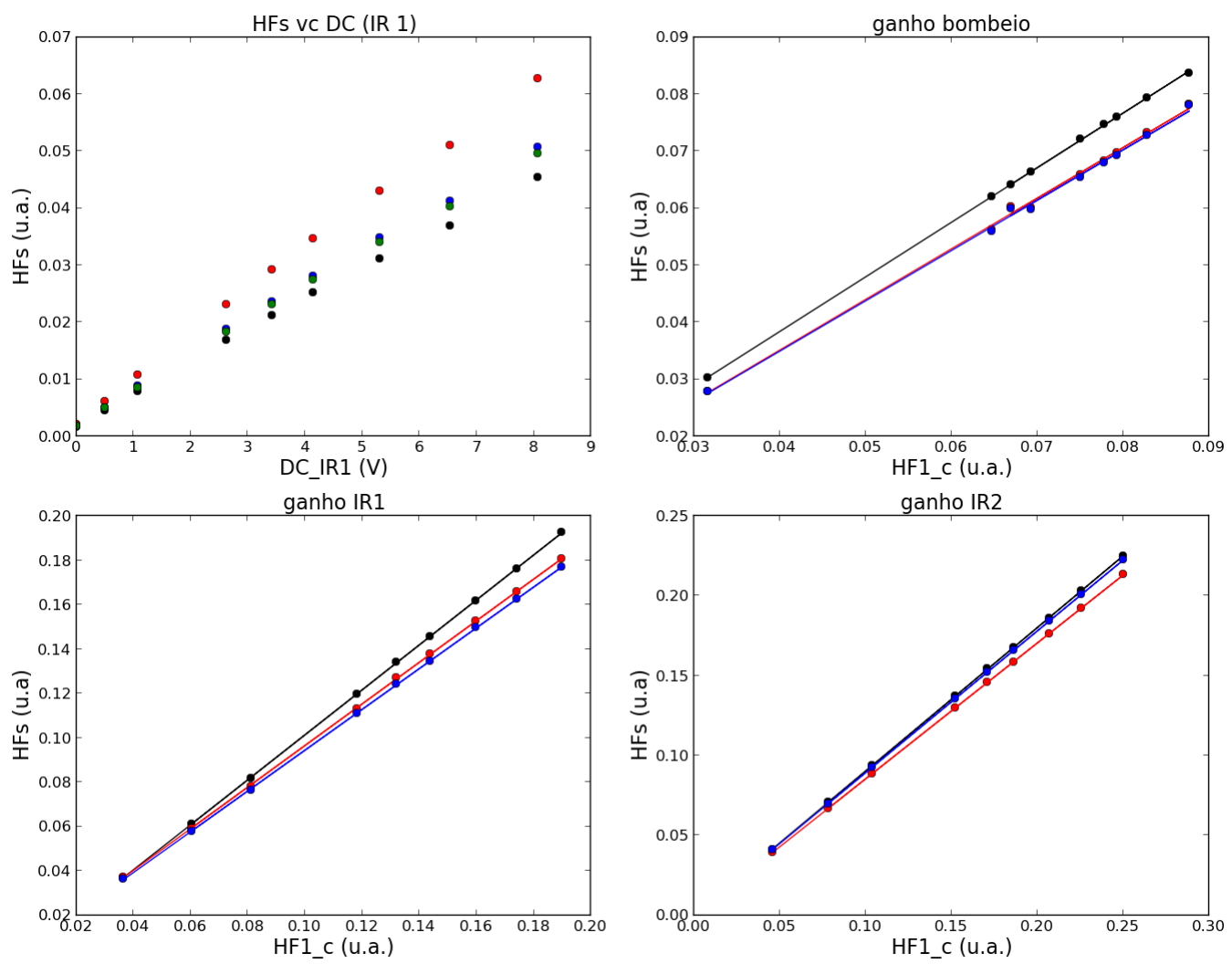

Figura 4.9: A figura no alto e á esquerda mostra as variâncias de cada canal de aquisição para um dos feixes gêmeos em função da intensidade. As outras três figuras mostram o fator de proporcionalidade entre os sinais HF para cada um dos três campos. Nestes gráficos estão mostradas as raízes da variâncias de três canais de aquisição HF versus o quarto canal na condição em que há o DC dos quatro canais são iguais.

A terceira forma de detecção foi implementada com o objetivo de realizar a caracterização completa do sistema das bandas laterais, como discutido no capítulo 3. Este tipo de medida nos permite acessar também a parte imaginária da matriz espectral. Para isto, dividimos ao meio cada sinal proveniente dos fotodetectores e os demodulamos com dois osciladores locais em quadratura chamados componente cosseno e seno, também como discutido no capítulo3. Idealmente, estes 4 canais de detecção deveriam possuir mesmo ruído, pois estamos enviando a mesma intensidade de luz para cada um deles. Este não é necessariamente o caso, como mostrado na figura 4.9. mas esta possível diferença pode ser corrigida medindo-se cada canal de forma independente antes do experimento e extraindo um fator de compensação entre eles, que chamamos de ganho 
dos detetores. O shot noise é então calculado da mesma forma que em (4.1) para ambas
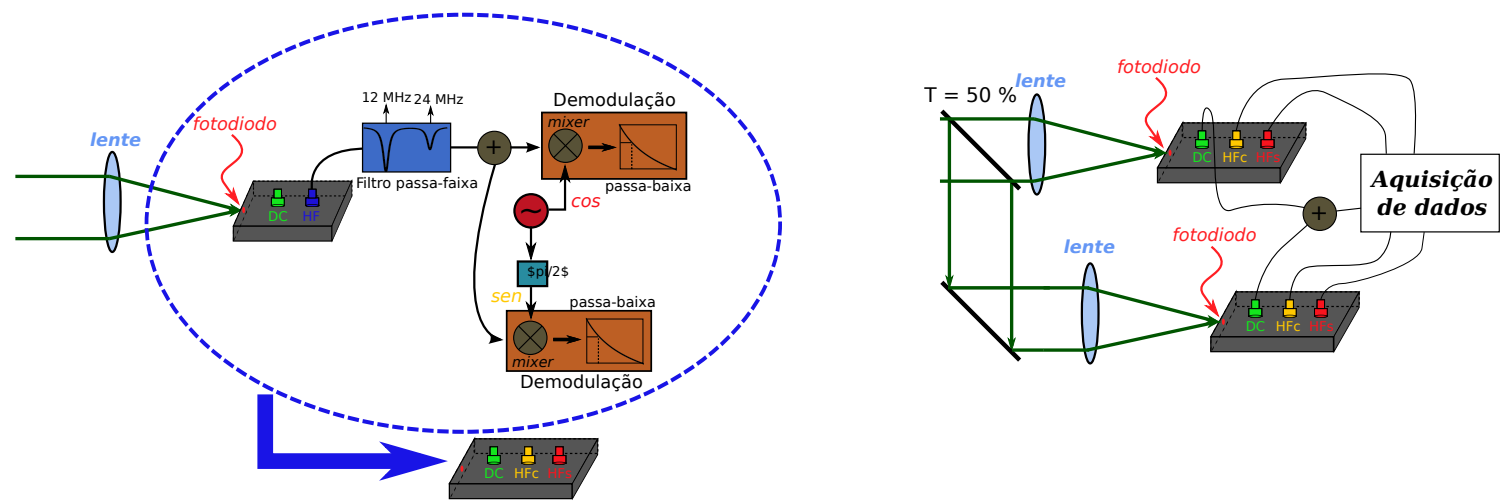

Figura 4.10: Terceiro sistema de detecção: na figura da esquerda, podemos ver como o sinal HF proveniente de cada fotodetector é dividido e demodulado com dois osciladores eletrônicos defasados de $90^{\circ}$. Na figura da esquerda, cada conjunto fotodetector + filtro + demoduladores, chamados de HFc e HFs é enviado para a aquisição.

as saídas HFc e HFs. Como o cálculo é feito após a calibração do ganho, estes valores devem coincidir.

\subsection{Estabilização de Frequência e Temperatura}

Uma parte essencial da nossa montagem experimental são os sistemas de travamento, ou estabilização, de frequência e temperatura. Ela é feita por meio de uma eletrônica de controle, sem a qual seria muito difícil observar o emaranhamento no OPO. Os parâmetros físicos que estabilizamos são a temperatura do cristal de KTP e as frequências de ressonância das cavidades de filtro e do OPO. A cavidade de filtro é travada na ressonância de modo que o campo transmitido seja máximo e o OPO também é travado na ressonância. Este travamento mantém a frequência do campo de bombeio igual à da ressonância da cavidade. Usamos a temperatura do cristal para ajustar a dessintonia dos feixes gêmeos e mantê-los em ressonância também. Os três sistemas de travamento podem ser resumidos de forma bem parecida. Eles consistem de cinco partes, como mostrada no diagrama 4.11; o sistema físico em questão, um detetor que converte a medida do parâmetro físico em tensão, o discriminante, o servo (que interpreta os sinais observados) e o atuador (que corrige os parâmetros do sistema para mantê-lo estabilizado).

O sistema físico representa o parâmetro que queremos estabilizar. No OPO, estes são a temperatura do cristal de KTP e a dessintonia da cavidade em relação ao campo 


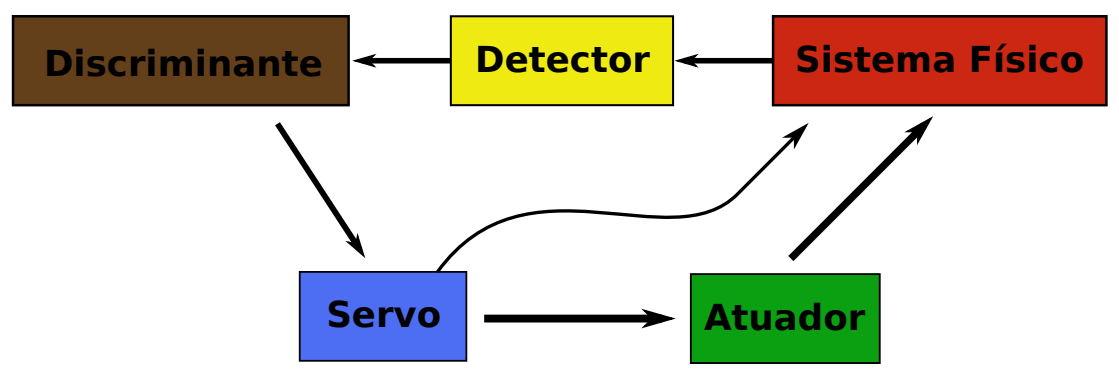

Figura 4.11: Figura esquemática mostrando as etapas do sistema de realimentação negativa usado para o travamento de frequência e temperatura.

de bombeio, ajustada pelo tamanho da mesma. Para a cavidade de filtro, ajustamos também a dessintonia da mesma em relação ao campo de entrada. O detetor nos fornece uma medida do parâmetro a ser estabilizado. Para os travamentos de frequência, medimos a luz refletida pelas cavidades, tanto do OPO quanto da cavidade de filtro, usando fotodiodos. Quando a cavidade estiver em ressonância, haverá um pico na transmissão e, consequentemente, um vale no sinal de reflexão. O objetivo é manter o tamanho da cavidade travado neste vale. Para a estabilização de temperatura, usamos um termistor, que converte temperatura em voltagem, como detector. O ponto ótimo neste caso é ajustado no próprio circuito de modo que a potência dos feixes gêmeos seja máxima.

Nossos sistemas de controle são baseados em uma malha de realimentação negativa. O circuito é desenhado para enviar um sinal no sentido oposto ao aumento de determinado parâmetro. No exemplo da temperatura, isto consiste em mandar um aviso para resfriar o cristal sempre que a temperatura se tornar maior que a ajustada e vice versa. O objetivo do discriminante é traduzir o sinal observado pelo detetor para algo que possa ser "entendido"pela eletrônica de controle. Para a estabilização da temperatura, o discriminante é dado pela subtração entre a temperatura ajustada e a medida, que possui sinal oposto à mudança do parâmetro. Em outras palavras, se a temperatura aumenta, a tensão gerada pelo discriminante é negativa e fará com que se esfrie o cristal. Ela é chamada sinal de erro e, em função da temperatura, consiste em uma reta que passa pela temperatura ajustada com coeficiente angular unitário. Olhando deste ponto de vista, o objetivo do discriminante é transformar o sinal medido pelo detetor em um sinal de erro. Em um caso geral, este não precisa ser uma reta, porém deve ter as seguintes propriedades: se anula no ponto em que se deseja estabilizar, é positivo quando o sistema se afasta deste ponto em um determinado sentido e é negativo quando o mesmo se afasta no outro sentido. 
Quando queremos travar uma cavidade no pico de transmissão, o sinal medido não pode mais ser associado diretamente com o sinal de erro. Se colocamos o zero de voltagem no vale da reflexão, sempre que o sistema sair deste ponto, a tensão no fotodiodo será positiva, independentemente de o tamanho da cavidade aumentar ou diminuir. Note que mesmo que o zero esteja em outro lugar, o sinal da tensão medida no fotodiodo não muda quando passamos pela ressonância. Neste ponto, fica clara a necessidade do papel desempenhado pelo discriminante. Para os travamentos de frequência, usamos dois métodos diferentes como discriminantes de frequência: o Pound-Drever-Hall [95] para a cavidade de filtro e o Dither-Lock para a cavidade do OPO. Pensando de forma simplificada, o Dither-Lock calcula a derivada do sinal de reflexão da cavidade. Acessamos as ressonâncias das cavidades varrendo o tamanho das mesmas por meio de uma cerâmica piezoelétrica (PZT), que muda de tamanho de acordo com a tensão aplicada. Para calcular a derivada do sinal, aplicamos uma modulação senoidal, gerada por um VCO (Voltage Controled Oscillator), de amplitude $\delta$ muito menor que a do sinal de varredura $\Delta$ e com frequência $\omega$ muito maior que a da varredura, $\omega_{s}$. A intensidade do campo refletido é função da soma destas contribuições,

$$
I\left(\Delta^{\prime}\right)=I(\Delta+\delta \cos (\omega t)) \approx I(\Delta)+\left.\frac{\partial I}{\partial \Delta^{\prime}}\right|_{\Delta^{\prime}=\Delta} \delta \cos (\omega t),
$$

onde consideramos o sinal de varredura como sendo um sinal DC, pois $\omega>>\omega_{s}$. $\mathrm{O}$ resultado é a soma entre a tensão original na parte DC e uma proporcional à derivada oscilando na frequência $\omega$. Para isolarmos a derivada, passamos esta por um circuito de demodulação como discutido no capítulo 3. O sinal medido é misturado com um sinal senoidal, retirado do mesmo $V C O$ e com fase relativa ajustável, $f=f_{0} \cos (\omega t+\phi)$. Após passagem pelo misturador, a tensão será

$$
V_{m}(\Delta)=\left.\frac{\partial I}{\partial \Delta^{\prime}}\right|_{\Delta^{\prime}=\Delta} \frac{f_{0} \delta}{2}[\cos (2 \omega t+\phi)+\cos \phi]
$$

Ao final, há um filtro passa-baixa com frequência de corte muito menor que $2 \omega$, resultando em um sinal de

$$
V_{\phi}(\Delta)=\left.\frac{\partial I}{\partial \Delta^{\prime}}\right|_{\Delta^{\prime}=\Delta} \frac{f_{0} \delta}{2} \cos \phi,
$$

que pode ser ajustado de acordo com a fase $\phi$ para ser proporcional à derivada ou ao negativo da mesma. Uma destas escolhas fará com que o sistema seja travado e a outra com que haja divergências. O servo é responsável por realimentar o sistema físico com algo que depende do sinal de erro. Em todos os travamentos usamos como servo um circuito, chamado PID, que realimenta a soma três sinais: um proporcional ao 

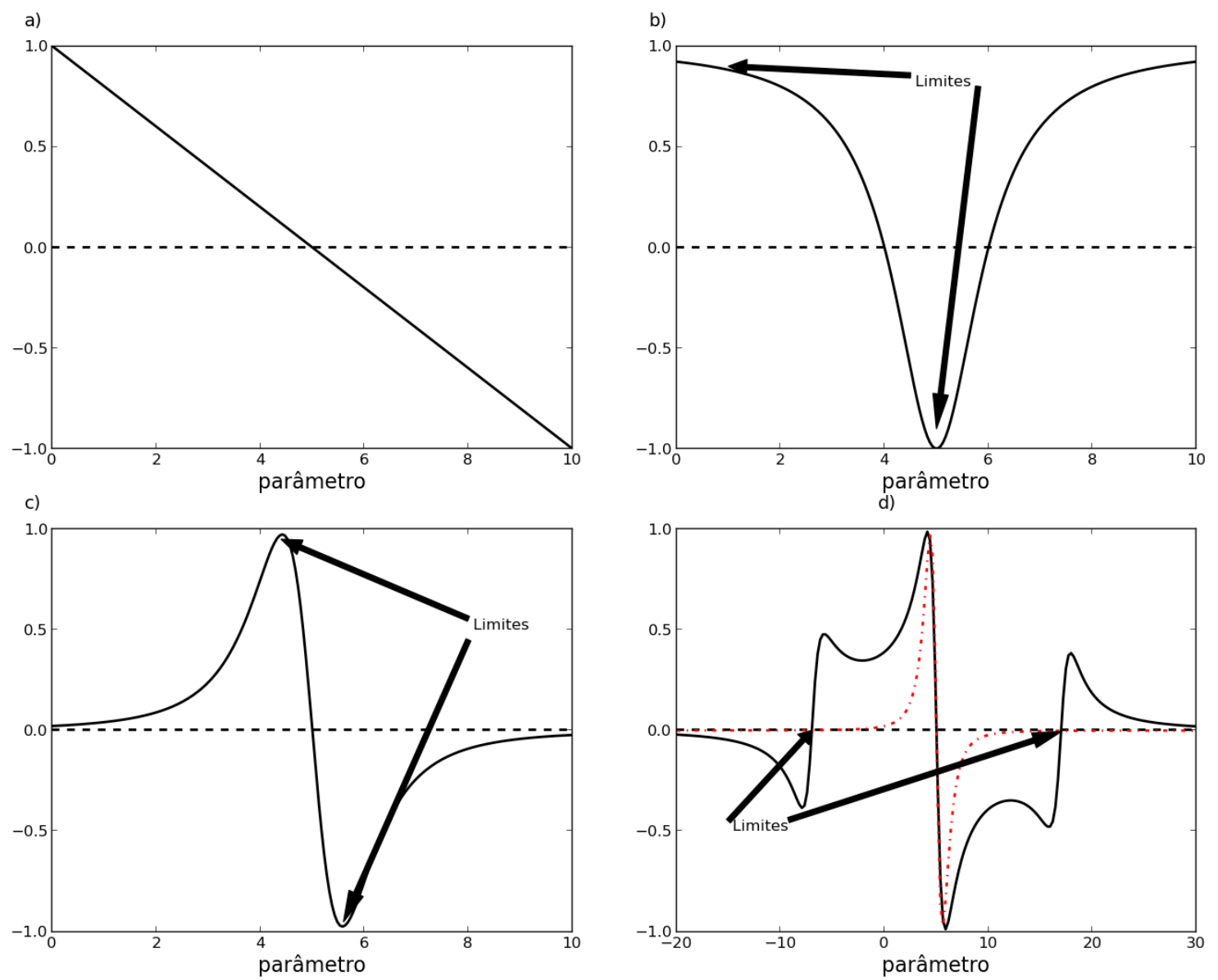

Figura 4.12: Exemplos de diferentes tipo de sinais de erro tendo como ponto ótimo $x 0=5$. A curva a) mostra o sinal linear como o que utilizamos para a estabilização da temperatura. Para este tipo de sinal de erro a estabilização não possui limites em relação à região de captura qualquer temperatura inicial iá convergir para $x 0$ se esperamos tempo suficiente. A curva b) mostra o campo refletido por uma cavidade Fabry-Perot. Podemos travar no meio da curva se usamos a lateral do pico como sinal de erro. Na curva c), é mostrada a derivada do pico de reflexão ou de transmissão de uma cavidade. Um sinal de erro como este é obtido com o método de Dither-Lock e é utilizado para o travamento do OPO. Para os sinais de erro b) e c), a região de captura é da ordem da largura de banda do pico e fora desta região, o sistema diverge em vez de ser estabilizado. Na curva d) está o sinal de erro obtido com o método Pound-Drever-Hall e uma comparação com o Dither-Lock. Note que a região de captura é bem maior, o que faz com que este método seja mais robusto.

sinal de erro original, outro proporcional à integral do mesmo e outro proporcional à sua derivada. O atuador é responsável por aplicar o sinal do servo de volta no sistema físico. Para o controle de temperatura, esse papel é desempenhado por um elemento peltier, que gera um gradiente de temperatura proporcional à voltagem aplicada. Ele é capaz de fazer ajustes finos na temperatura, pois permite tanto esfriar quanto esquentar o sistema cuja temperatura se deseja controlar. Para as cavidades, o atuador é um PZT e um dos espelhos da cavidade está montado montado o mesmo. Isto nos permite 
controlar o tamanho da cavidade através da voltagem com uma grande precisão ${ }^{9}$.

Após ter discutido neste capítulo as técnicas que utilizamos para controlar e preparar os nossos estados, passarei, no próximo, ao aparato teórico que usamos para caracterizar, baseado nas medidas de quadratura que podemos fazer, o emaranhamento em nosso sistema de variáveis contínuas. Nele também discutirei a parte teórica do nosso estudo sobre a robustez do emaranhamento em variáveis contínuas, focando em sistemas formados por apenas dois modos.

\footnotetext{
${ }^{9}$ Algo da ordem de unidades de nanômetro.
} 


\section{$5 \quad$ Emaranhamento em Variáveis Continuas}

Neste capítulo, discutirei o emaranhamento em maior profundidade. As três primeiras seções contém resultados já conhecidos na literatura e são revisados aqui, pois são parte importante da base teórica utilizada no nosso trabalho. Na quarta seção, discuto resultados originais sobre a investigação da dinâmica do emaranhamento frente a perdas por transmissão que estão publicados em [27, 96]

\subsection{Emaranhamento}

Os estados separáveis são definidos como todos aqueles que podem ser preparados usando somente operações locais e comunicação clássica (LOCC) - do inglês Local Operations and Classical Communication. Como dito anteriormente, os estados emaranhados são todos que não são separáveis [42, 97]. Para entender melhor esta definição, vou discutir brevemente de onde ela surge. Considere que duas pessoas, Alice e Bob, estejam de posse cada uma de um sistema físico, A e B, e que possam se comunicar através de um canal clássico ${ }^{1}$. Cada uma delas pode preparar seu respectivo sistema em um número arbitrário, $n$, de estados diferentes. Considere também que Alice possua um gerador de números aleatórios que cria uma sequência de números inteiros $i \in\{1,2, \ldots n\}$ com probabilidades $p_{1}, p_{2}, \ldots$ e $p_{n}$. Para cada " $i$ " gerado, Alice prepara o seu sistema em um estado $\hat{\rho}_{i}^{A}$, previamente combinado entre ela e Bob, e envia uma mensagem para que Bob prepare o estado $\hat{\rho}_{i}^{B}$. A cada vez que o gerador mostrar o valor " $i$ ", o estado conjunto de Alice e Bob será $\hat{\rho}_{i}^{A} \otimes \hat{\rho}_{i}^{B}$. Após um grande número de repetições, o estado resultante será a combinação destes estados com suas respectivas

\footnotetext{
${ }^{1}$ Um canal clássico é qualquer meio de comunicação que transmita bits. Podendo ser uma linha de telefone, código morse, sinal de fumaça etc.
} 
probabilidades,

$$
\hat{\rho}_{\text {sep }}=\sum_{i=1}^{n} p_{i} \hat{\rho}_{i}^{A} \otimes \hat{\rho}_{i}^{B},
$$

Estados desta forma são chamados estados separáveis e são tipicamente correlacionados, pois Bob prepara um estado distinto e pré-determinado para cada um criado por Alice. Entretanto, estas correlações são fruto somente da comunicação clássica, pois os dois sistemas não interagem. Os estados emaranhados são os que não podem ser descritos da forma (5.1). Como $0 \leq p_{i} \leq 1$, a combinação linear (5.1) é uma soma convexa de operadores densidade descorrelacionados, o que significa que os estados separáveis formam um subespaço convexo do espaço de estados físicos [44, 98]. Esta é uma propriedade geométrica usada nos primeiros critérios de emaranhamento, baseados em testemunhas de emaranhamento [99], e em algumas medidas de emaranhamento [100]

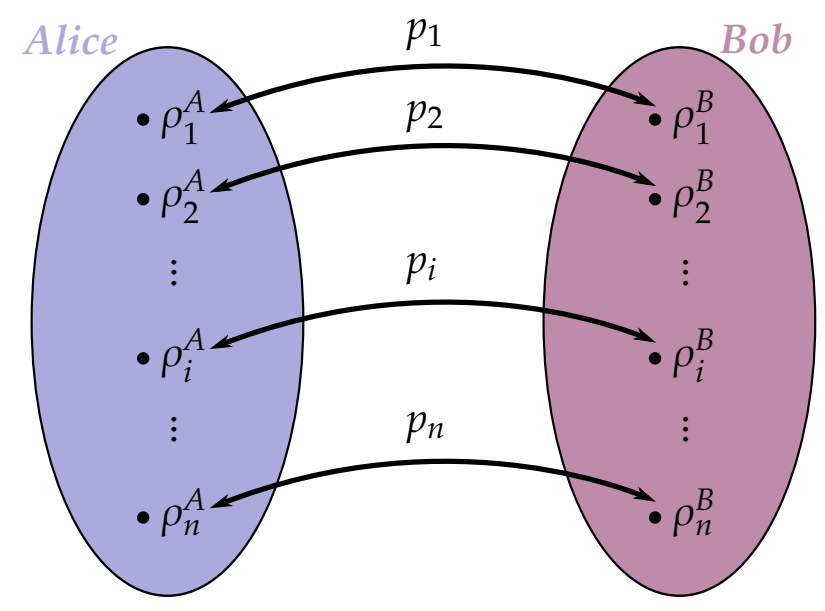

Figura 5.1: Diagragma mostrando as LOCC. Os pontos pretos representam os estados que podem ser criados por Alice e Bob usando apenas operações locais. Em cada um deles, Alice e Bob criam um estado pré-determinado e condicionado ao valor de uma variável aleatória. As setas representam a comunicação clássica, com a qual Alice e Bob compartilham o valor da variável aleatória.

A definição de emaranhamento, entretanto, não é operacional, pois não fornece um procedimento que permita dizer se determinado estado pode ser escrito da forma 5.12. Por isso há a necessidade de se utilizar critérios de emaranhamento, que são procedimentos diretamente aplicáveis no contexto experimental. Existem atualmente diversos critérios para um amplo espectro de aplicações [101, 89, 102, 103, 104, 105] e discutirei alguns deles, que são usados explicitamente neste trabalho.

O critério de Peres-Horodecki, também conhecido como PPT (do inglês - Positivity

\footnotetext{
${ }^{2}$ De fato, checar isso diretamente é um problema difícil do ponto de vista computacional, pois, em princípio, há infinitas bases nas quais é possível decompor um operador densidade.
} 
under Partial Transposition) [101, 99], se baseia no fato de que a transposição do operador densidade em relação a apenas uma das partes pode não manter a positividade do mesmo. Como a transposição de um operador é uma transformação que conserva os autovalores, a positividade do mesmo é sempre mantida quando o sistema global é transposto. Transformações com este tipo de característica são conhecidas como mapas positivos, mas não completamente positivos ${ }^{3}$. Os requisitos para que $\rho$ seja um operador densidade são:

- que seja hermitiano, o que implica que pode ser diagonalizado por uma mudança de base e seus autovalores são reais.

- que seus autovalores sejam positivos ou nulos, ou seja, $\hat{\rho}$ é um operador positivo semidefinido, característica respresentada por $\hat{\rho} \geq 0$.

- que possua traço unitário.

Todos esses requisitos são necessários para que os autovalores de $\hat{\rho}$ possam ser interpretados como probabilidades. Se aplicamos a operação de transposição em $\hat{\rho}$,

$$
\hat{\rho} \longrightarrow \tilde{\hat{\rho}}=(\hat{\rho})^{T}
$$

o estado resultante, $\tilde{\hat{\rho}}$, é hermitiano e, como a transposição preserva os autovalores, é também positivo e de traço unitário. Portanto, $\tilde{\hat{\rho}}$ continua sendo um operador densidade. Entretanto, se a tranposição for aplicada em apenas uma das partes, a hermiticidade e a unitariedade do traço ainda são mantidas, mas alguns autovalores podem ser negativos.

Quando $\hat{\rho}$ é um estado separável e transpomos, por exemplo, o sistema de Bob,

$$
\hat{\rho} \longrightarrow \hat{\rho}^{P T}=\sum_{i} \hat{\rho}_{i}^{A} \otimes\left(\hat{\rho}_{i}^{B}\right)^{T_{B}}
$$

Neste caso, $\hat{\rho}^{P T}$ ainda é um operador densidade, pois a transposição parcial vai atuar em $\rho_{i}^{B}$ como uma transposição global. Sendo assim, se $\hat{\rho}^{P T}<0$ após a transposição parcial, o estado inicial não pode ser escrito na forma (5.1) e, consequentemente, é emaranhado. Note que $\hat{\rho}^{P T} \geq 0$ é uma condição necessária para a separabilidade, logo, $\hat{\rho}^{P T}<0$ é suficiente para o emaranhamento, qualquer que seja $\hat{\rho}$. Apenas se pode provar que ela

\footnotetext{
${ }^{3}$ Mapas positivos são aqueles que transformam operadores positivos e hermitianos em outros operadores também positivos e a hermitianos. Mapas completamente positivos são mapas positivos com uma restrição a mais: eles devem manter a positividade e hermiticidade também quando aplicados a qualquer extensão do espaço [44, 106].
} 
é também necessária em alguns poucos casos [99, 107, 102 $]_{4}^{4}$. Como os autovalores do operador densidade são probabilidades, a transposição parcial mapeia alguns estados emaranhados em estados com probabilidades negativas. Isso corresponde a dizer que a transposição parcial é uma transformação não-física, pois pode mapear estados físicos em não-físicos 5 . Por este motivo, a transposição parcial não pode ser implementada no laboratório, mas somente aplicada após a reconstrução de $\hat{\rho}$.

Os exemplos mais simples de aplicação do critério PPT ocorrem em estados de 2 qtbis. Podemos testar o emaranhamento, por exemplo, de um dos estados de Bell 2.2). O operador densidade para $\left|\Phi_{+}\right\rangle=\frac{1}{\sqrt{2}}\left[\left|0_{A}\right\rangle \otimes\left|0_{B}\right\rangle \pm\left|1_{A}\right\rangle \otimes\left|1_{B}\right\rangle\right]$ expandido na base conjunta, $\left\{\left|0_{A}, 0_{B}\right\rangle,\left|0_{A}, 1_{B}\right\rangle,\left|1_{A}, 0_{B}\right\rangle,\left|1_{A}, 1_{B}\right\rangle\right\}$ é

$$
\hat{\rho}=\left|\Phi_{+}\right\rangle\left\langle\Phi_{+}\right|=\frac{1}{2}\left(\begin{array}{cccc}
1 & 0 & 0 & 1 \\
0 & 0 & 0 & 0 \\
0 & 0 & 0 & 0 \\
1 & 0 & 0 & 1
\end{array}\right) .
$$

A transposição parcial irá trocar valores da base do subsistema $B$ fazendo com que $1_{B} \leftrightarrow 0_{B}$. Portanto, o operador densidade parcialmente transposto é

$$
\hat{\rho}^{P T}=\frac{1}{2}\left(\begin{array}{cccc}
1 & 0 & 0 & 0 \\
0 & 0 & 1 & 0 \\
0 & 1 & 0 & 0 \\
0 & 0 & 0 & 1
\end{array}\right) \text {. }
$$

Os quatro autovalores desta matriz são $\lambda_{1}=\lambda_{2}=\lambda_{3}=0.5$ e $\lambda_{4}=-0.5$. Note que eles ainda são reais e $\sum_{i} \lambda_{i}=1$, pois $\hat{\rho}^{P T}=\left[\hat{\rho}^{P T}\right]^{\dagger}$ e $\operatorname{tr}\left[\hat{\rho}^{P T}\right]=1$, mas $\hat{\rho}^{P T}<0$ indicando emaranhamento. De posse de $\hat{\rho}$, o critério PPT fornece uma receita para testar se há emaranhamento no sistema. Neste exemplo, a verificação pode ser feita rapidamente, porém a medida que a dimensão do espaço de Hilbert aumenta, a aplicação deste critério vai ficando cada vez mais complexa. Para sistemas de dimensão infinita, como o que trabalhamos, é inviável medir o estado $\hat{\rho}$. Em geral utilizamos representações de quase-probabilidade, que descrevem o sistema no espaço de fase, como substitutos

\footnotetext{
${ }^{4}$ Estes consistem em dois casos com baixa dimensão do espaço de Hilbert, sistema do tipo $2 \otimes 2$ e $2 \otimes 3$ [99], e um com dimensão infinita: estados gaussianos quando há somente um modo em uma das partes [107, 102].

${ }^{5}$ Formalmente, transformações físicas devem manter tanto a positividade quanto a hermiticidade do operador densidade global quando aplicada em qualquer subsistema que o componha. As transformações que satisfazem essas condições são conhecidas como mapas completamente positivos ou operadores de Kraus [106].
} 
para $\hat{\rho}[73,108]$.

\subsection{Variáveis Contínuas}

Sistemas de variáveis contínuas são formados por observáveis cujos espectros podem assumir valores contínuos. Em geral, estes observáveis obedecem às relações de comutação para posição e momento,

$$
\left[\hat{q}_{i}, \hat{p}_{j}\right]=2 i \delta_{i j}, 6
$$

em que $i, j=1,2, \ldots, n$ para um sistema composto por $n$ modos. Estes operadores podem ser arranjados no vetor de operadores $\hat{\xi}=\left(\hat{q}_{1}, \hat{p}_{1}, \ldots, \hat{q}_{\alpha}, \hat{p}_{\alpha}, \ldots, \hat{q}_{n}, \hat{p}_{n}\right)$, onde $\hat{q}_{\alpha}$ e $\hat{p}_{\alpha}$ são variáveis dinâmicas que fazem o papel posição e momento, respectivamente, de modo que o espaço de fase seja formado pelos espectros destes operadores. Se tratando de um sistema quântico, não é possível definir um ponto no espaço de fase devido às relações de incerteza, porém, ainda assim, podemos usá-lo para estudar a dinâmica dos sistemas quânticos [108, 109].

As variáveis contínuas são a descrição natural para o sistema com que trabalhamos, pois as flutuações de quadratura que medimos se comportam como variáveis contínuas de média nula. As médias são dadas por $\left\langle\hat{\xi}_{i}\right\rangle=\operatorname{Tr}\left(\hat{\xi}_{i} \rho\right)$, onde $\rho$ é o operador densidade do sistema global. As flutuações em relação à média são definidas como $\delta \hat{\xi}_{i}=\hat{\xi}_{i}-\left\langle\hat{\xi}_{i}\right\rangle$ de modo que $\left\langle\delta \hat{\xi}_{i}\right\rangle=0$. Por conveniência, podemos escolher distribuições de quaseprobabilidade ${ }^{7}[73$, 87, 110] para descrever sistemas quânticos no lugar do operador densidade. Estas distribuições tem como principal propriedade o fato de médias sobre operadores se tornarem médias estatísticas, tendo as mesmas como peso,

$$
\operatorname{Tr}\left[f\left(\hat{a}, \hat{a}^{\dagger}\right) \rho\right]=\int d^{2} \alpha f\left(\alpha, \alpha^{*}\right) P^{(o)}\left(\alpha, \alpha^{*}\right),
$$

onde $f\left(\hat{a}, \hat{a}^{\dagger}\right)$ é uma função qualquer de $\hat{a}$ e $\hat{a}^{\dagger}$ em alguma ordenação ${ }^{8}$. Desta forma, $P^{(o)}\left(\alpha, \alpha^{*}\right)$ é uma representação do operador densidade para um sistema que possui apenas um modo. As quantidades $\alpha$ são representações no plano complexo (ou no diagrama de Fresnel) que tem como partes real e imaginária os autovalores de $\hat{q}$ e $\hat{p}$,

\footnotetext{
${ }^{6} \mathrm{O} 2$ é colocado aqui somente por conveniência. Esta escolha fará com que os ruídos de fase e intensidade de um estado de vácuo sejam iguais a 1.

${ }^{7}$ Chamadas assim porque não possuem todas as propriedades inerentes às distribuições de probabilidades, como a positividade.

${ }^{8}$ Por exemplo, $f\left(\hat{a}, \hat{a}^{\dagger}\right)=\hbar \omega\left(\hat{a} \hat{a}^{\dagger}+\hat{a}^{\dagger} \hat{a}\right) / 2$ representa a energia de um oscilador harmônico livre na ordenação simétrica.
} 
respectivamente. Uma mesma função de operadores que não comutam podem estar em diversas ordenações, como por exemplo, $f\left(\hat{a}, \hat{a}^{\dagger}\right)=\hat{a} \hat{a}^{\dagger}+\hat{a}^{\dagger} \hat{a}=2 \hat{a} \hat{a}^{\dagger}-1=2 \hat{a}^{\dagger} \hat{a}+1 \mathrm{em}$ ordens simétrica, antinormal e normal, respectivamente. Cada uma destas correspondem a funções diferentes definida para números complexos e este fato se reflete nas distribuições de quase-probabilidade. Para cada ordenação possível dos operadores $a$ e $a^{\dagger}$ haverá uma representação de quase-probabilidade. As ordenações mais comuns são as ordens normal, anti-normal e simétrica, que são representadas pelas distribuições $P$ de Glauber-Sudarshan, $Q$ de Hussimi e pela função de Wigner, $W$ [73, 110]. Esta última é a distribuição de quase-probabilidade que será usada neste trabalho.

a)

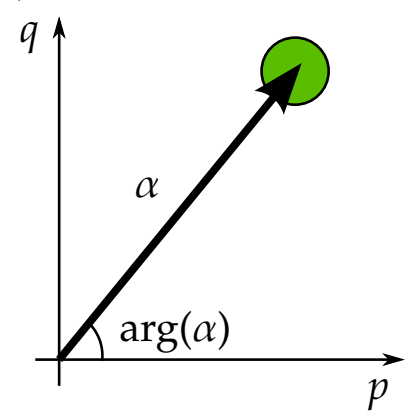

b)

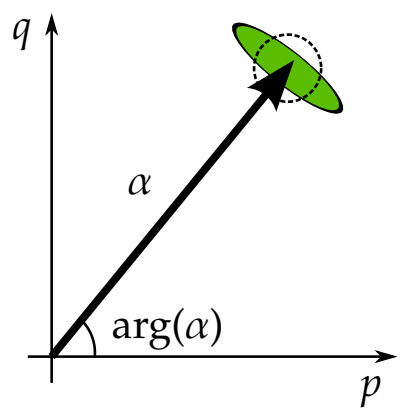

c)

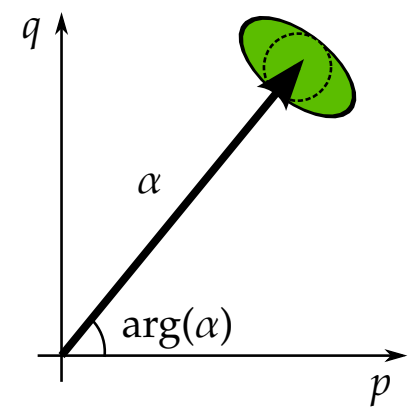

Figura 5.2: Digramas de Fresnel representando o espaço de fase quântico. Ele é especialmente útil quando lidamos com estados gaussianos, para os quais área em verde é o corte na função de Wigner na altura 1/e do máximo. Os três estados representados possuem o mesmo valor médio $\alpha$, mas possuem ruídos diferentes. Na figura a) é mostrado um estado coerente, em b) um estado de squeezing e em c) um estado com ruído de amplitude igual ao shot noise e excesso de ruído na fase.

A função de Wigner que representa o operador densidade $\rho$ e é definida em função das variáveis canônicas, como [111]

$$
W(q, p)=\frac{1}{(\pi \hbar)^{n}} \int_{-\infty}^{\infty} d^{n} y\langle q-y|\rho| q+y\rangle e^{2 i p y / \hbar}
$$

onde $|q \pm y\rangle=\left|q_{1} \pm y_{1}\right\rangle \otimes\left|q_{2} \pm y_{1}\right\rangle \cdots \otimes\left|q_{n} \pm y_{n}\right\rangle$ e $p y=\sum_{i} p_{i} y_{i}$. Ela tem a propriedade de que a integração de qualquer combinação de $q$ e $p$ fornece a densidade de probabilidade de se medir a combinação conjugada. Em função de $\alpha=(q+i p) / 2$ e $\alpha^{*}$, a função de Wigner se torna [73]

$$
\begin{aligned}
W\left(\alpha, \alpha^{*}\right) & =\frac{1}{\left(\pi^{2} \hbar\right)^{n}} \int_{-\infty}^{\infty} d^{2 n} \beta e^{-i\left(\beta^{*} \alpha+\beta \alpha^{*}\right)} \chi\left(\beta, \beta^{*}\right), \\
\chi\left(\beta, \beta^{*}\right) & =\operatorname{Tr}\left[e^{i\left(\beta^{*} \hat{a}+\beta \hat{a}^{+}\right)} \hat{\rho}\right] .
\end{aligned}
$$

Ela é negativa para alguns estados quânticos, como os estados número, porém se 
mantém positiva para outros, como os estados comprimidos (squeezing). Em se tratando de estados puros, a função de Wigner é positiva somente para estados gaussianos [112]. O diagrama de Fresnel mostrado na figura 5.2 é um corte da função de Wigner em um plano paralelo ao plano $W(p, q)=0$. Utilizamos a função de Wigner em nossos cálculos, pois ela é a distribuição de quase-probabilidade correspondente à ordem simétrica.

A ordem simétrica tem outra característica que a diferencia das outras: somente nela uma matriz de covariâncias entre operadores hermitianos que não comutam será real. Isto é consequência direta do fato de que, para qualquer conjunto de operadores hermitianos $\hat{\xi}=\left(\hat{\xi}_{1}, \hat{\xi}_{2}, \ldots, \hat{\xi}_{n}\right)$,

$$
V_{i j}=\left\langle\hat{\xi}_{i} \hat{\xi}_{j}\right\rangle=\left\langle\hat{\xi}_{j} \hat{\xi}_{i}\right\rangle^{*}=V_{j i}^{*}
$$

Se assumimos que $\left[\hat{\xi}_{i}, \hat{\xi}_{j}\right] \neq 0$ e definimos uma ordenação $V_{i j}^{\lambda}=\left\langle\lambda \hat{\xi}_{i} \hat{\xi}_{j}+(1-\lambda) \hat{\xi}_{j} \hat{\xi}_{i}\right\rangle$, com $0 \leq \lambda \leq 1, V_{i j}^{\lambda}=V_{j i}^{\lambda}$ se, e somente se, $\lambda=1 / 2$, o que corresponde à ordem simétrica. Por este motivo, os estados gaussianos em variáveis contínuas são definidos a partir da função de Wigner [113]. Um estado gaussiano de $n$ modos é aquele cuja função de Wigner é dada por

$$
W(x)=\frac{1}{\pi^{n} \sqrt{\operatorname{det} V}} \mathrm{e}^{-\frac{1}{2} x^{T} V^{-1} x},
$$

onde $V$ é a matriz de covariâncias, com componentes $V_{i j}=\left\langle\hat{\xi}_{i} \hat{\xi}_{j}+\hat{\xi}_{j} \hat{\xi}_{i}\right\rangle / 2$. Podemos considerar todas as variáveis com a média nula, pois os valores médios podem ser retirados por deslocamentos no espaço de fase [114]. Eles descrevem somente a dinâmica clássica do sistema e não influenciam o emaranhamento. Uma quantidade importante em diversas situações é a pureza, definida como $\mu=\operatorname{tr}\left[\hat{\rho}^{2}\right]$. Para estados puros, $\mu=1$ e ela diminui quanto mais misto for o estado. Para estados gaussianos, a pureza é [115]

$$
\mu=\frac{1}{\sqrt{\operatorname{det} V}} .
$$

O critério PPT utiliza a negatividade do operador densidade parcialmente transposto para atestar o emaranhamento. Essa negatividade não é nada mais que uma violação da condição de fisicalidade do estado final. Em um sistema de variáveis contínuas, o operador densidade possui dimensão infinita. Por este motivo, é mais simples associar a fisicalidade do estado às relações de incerteza [116]. As mesmas se baseiam na positividade da matriz $\beta$, cujas entradas são dadas por

$$
\beta_{i j}=\left\langle\delta \hat{\xi}_{i} \delta \hat{\xi}_{j}\right\rangle
$$


Sendo $\beta$ uma matriz hermitiana, esta pode ser diagonalizada por uma transformação unitária. Como esta transformação em $\beta$ equivale a uma rotação - ou uma mudança de base - em $\delta \xi$,

$$
\beta_{i j}^{\prime}=\left\langle\left(\delta \hat{\xi}_{i}^{\prime}\right)^{2}\right\rangle \delta_{i j}
$$

na base diagonal. Portanto, $\beta$ é uma matriz positiva e que pode ser escrita como

$$
\begin{aligned}
\beta= & V+i \Omega \geq 0, \\
V_{i j}=\frac{\left\langle\left\{\delta \hat{\xi}_{i}, \delta \hat{\xi}_{j}\right\}\right\rangle}{2} & i \Omega_{i j}=\frac{\left\langle\left[\delta \hat{\xi}_{i}, \delta \hat{\xi}_{j}\right]\right\rangle}{2} \\
\text { onde } \Omega=\left[\begin{array}{cc}
J & 0 \\
0 & J
\end{array}\right], & J=\left[\begin{array}{cc}
0 & 1 \\
-1 & 0
\end{array}\right] .
\end{aligned}
$$

A condição (5.16) é uma generalização das relações de incerteza [113] $]^{9}$ Em um sistema clássico, uma matriz de covariâncias deve ser simétrica e positiva ${ }^{10}$, porém, em um sistema quântico, há uma restrição maior dada por (5.16).

\subsection{Transformações Simpléticas e PPT em Variáveis Contínuas}

Aplicar o critério PPT diretamente em sistemas de variáveis contínuas é complicado e inconveniente, pois o espaço de Hilbert possui dimensão infinta. Como a negatividade dos autovalores é usada para atestar que o estado parcialmente transposto é não-físico, é possível aplicar o critério de emaranhamento usando a violação das relações de incerteza generalizadas (5.16) com a mesma finalidade. Ao contrário do que ocorre com a negatividade do autovalores do operador densidade, a violação desta relação é somente uma condição suficiente para que o estado seja não-físico. Ela se torna também um condição necessária somente para estados gaussianos [102].

Em variáveis contínuas, a transposição parcial age como uma reversão temporal em um dos subsistemas [107] - ela muda o sinal do momento, mas não afeta a posição, $\left(\hat{q}_{\alpha}, \hat{p}_{\alpha}\right) \longrightarrow\left(\hat{q}_{\alpha},-\hat{p}_{\alpha}\right)$ ou $\tilde{\hat{\xi}}=\Lambda \hat{\xi} \operatorname{com} \Lambda=\operatorname{diag}(1,1, \ldots, 1,1, \ldots, 1,-1, \ldots, 1,-1) 11$. Isto pode

\footnotetext{
${ }^{9}$ No caso descorrelacionado, onde $V_{i j}=\sigma_{i}^{2} \delta_{i j}$, com $\sigma_{i}$ sendo o ruído, resgatamos a forma bem conhecida, $\sqrt{\left\langle\left(\delta p_{i}\right)^{2}\right\rangle\left\langle\left(\delta q_{i}\right)^{2}\right\rangle} \geq 1$, fazendo $\operatorname{det}(\beta) \geq 0$.

${ }^{10}$ Matematicamente, o ruído em qualquer uma das variáveis poderia ser nulo no caso descorrelacionado e para uma precisão arbitrariamente alta. Esta matriz também deve satisfazer a desigualdade de Cauchy-Schwarz, $V_{i j} \leq V_{i i} V_{j j}$.

${ }^{11}$ Os primeiros $d$ modos, presentes em um dos subsistemas, não sofrem modificações. Enquanto isso, a reversão temporal muda o sinal de cada $\hat{p}_{\alpha}$ para todos os $n-d$ modos presentes no subsistema transposto.
} 
ser derivado usando a expressão (5.8) para a função de Wigner, pois como

$$
\left\langle a\left|\hat{\rho}^{T}\right| b\right\rangle=\langle b|\hat{\rho}| a\rangle \quad \Longrightarrow \tilde{W}(q, p) \stackrel{P T}{\longrightarrow} W(q, p) .
$$

Portanto, o critério PPT será a condição (5.16) aplicada à matriz de covariâncias após a transposição parcial,

$$
\tilde{V}+i \Omega \geq 0, \quad \operatorname{com} \quad \tilde{V}=\Lambda V \Lambda .
$$

Esta é a condição de existência física para o estado após a transposição parcial.

\subsubsection{Transformações Simpléticas}

Neste ponto, entra o conceito de transformações simpléticas. Na Mecânica Clássica, elas são as transformações no espaço de fase que mantêm o parênteses de Poisson invariante ${ }^{12}$ e representam todas as transformações físicas possíveis em um sistema fechado [75, 117]. No contexto quântico, as transformações simpléticas são todas aquelas que, quando atuando no espaço de fase, mantém as relações de comutação invariantes 13 ,

$$
\Omega=S \Omega S^{T}
$$

Entretanto, diferente do que ocorre em sistemas clássicos, elas não são as transformações mais gerais possíveis em um sistema quântico fechado. Elas somente correspondem a transformações unitárias gaussianas [118]. Em um estado sujeito à transformação gaussiana unitária, cada operador no espaço de fase vai evoluir conforme

$$
\hat{\xi}_{k}(t)=\mathrm{e}^{i \hat{H} t} \hat{\xi}_{k} \mathrm{e}^{-i \hat{H} t}, \quad \text { onde } \quad \hat{H}=\sum_{i j} h_{i j} \hat{\xi}_{i} \hat{\xi}_{j} \quad \text { e } \quad h_{i j}^{*}=h_{j i}
$$

Esta última igualdade é consequência da hermiticidade de $H$ no espaço de Hilbert, o que faz com que a matriz $h$, com componentes dadas por $h_{i j}$, também seja hermitiana. A equação de Heisenberg correspondente a $\xi_{k}(t)$ é

$$
\frac{d \hat{\xi}_{k}(t)}{d t}=i\left[\hat{H}, \hat{\xi}_{k}(t)\right]=-2 \sum_{i}\left[\sum_{j} h_{i j}^{R} \Omega_{j k}\right] \hat{\xi}_{i}(t),
$$

\footnotetext{
${ }^{12}$ Em outras palavras, elas mantém a forma das equações de Hamilton.

${ }^{13}$ Que podem ser vistas como o equivalente quântico dos parenteses de Poisson na Mecânica Clássica.
} 
onde $h^{R}=h+h^{*}$ é uma matriz simétrica e $\Omega$, definida em (5.17), é antissimétrica. A solução matricial para este sistema de equações é

$$
\hat{\xi}(h)=S(h) \hat{\xi}, \quad \text { com } S(h)=\mathrm{e}^{-2 h^{R} \Omega},
$$

onde a dependência temporal foi retirada, pois pode ser absorvida em $h^{R}$. Note que a transformação depende apenas da parte real da matriz $h$, o que faz com que $H$ possa ser tomado na ordenação simétrica sem perda de generalidade. Para que $S(h)$ seja uma transformação simplética basta satisfazer (5.21). Isto pode ser verificado diretamente, pois - devido ao fato de que $\Omega^{2}=-\mathcal{I}$, onde $\mathcal{I}$ é a matriz identidade $-S(h)^{T}=\mathrm{e}^{2 \Omega h^{R}} \mathrm{e}$, consequentemente, $\Omega S(h)^{T}=S(h)^{-1} \Omega$. As transformações gaussianas mais comuns em ótica quântica são casos particulares de $H$,

squeezing de fase $: h^{R}=\left(\begin{array}{cc}0 & \lambda \\ \lambda & 0\end{array}\right), \quad$ evolução livre $: h^{R}=\left(\begin{array}{cc}\theta & 0 \\ 0 & \theta\end{array}\right)$,

$$
\text { EPR }: h^{R}=\left(\begin{array}{cccc}
0 & 0 & \lambda & 0 \\
0 & 0 & 0 & -\lambda \\
\lambda & 0 & 0 & 0 \\
0 & -\lambda & 0 & 0
\end{array}\right) \text {, divisor de feixes : } h^{R}=\left(\begin{array}{cccc}
0 & 0 & \theta & 0 \\
0 & 0 & 0 & \theta \\
\theta & 0 & 0 & 0 \\
0 & \theta & 0 & 0
\end{array}\right) \text {, }
$$

onde $\lambda$ representa o fator de squeezing no primeiro exemplo e fator de squeezing de dois modos no $h^{R}$ para o estado EPR 14 . Na evolução livre, $\theta=\omega t$, onde $t$ é o tempo de evolução e está relacionado aos coeficientes de transmissão e reflexão por $T=\cos ^{2} \theta$ e $R=\operatorname{sen}^{2} \theta$ no divisor de feixes.

As transformações simpléticas mapeiam estados físicos (não-físicos) em outros estados físicos (não-físicos), conservando a pureza global e a gaussianidade dos estados. Algo importante de ressaltar é que as transformações simpléticas, como mostrado explicitamente em (5.23), atuam no espaço de fase e não no espaço de Hilbert. Por este motivo, mesmo que a dinâmica gaussiana dos operadores no espaço de fase seja exatamente a mesma que ocorre em sistemas clássicos, a dinâmica do estado quântico difere daquela do estado clássico, pois a primeira ocorre no espaço de Hilbert enquanto a segunda ocorre no espaço de fase. As transformações simpléticas são importantes neste contexto porque constituem uma forma simples de testar a fisicalidade de um determinado estado [118]. Se aplicamos uma transformação simplética na relação (5.16), apenas $V$ mudará, mas a fisicalidade do estado original será preservada pela transformação. Desta forma, testar a validade de (5.16) se resume a checar se os

\footnotetext{
${ }^{14}$ Por uma questão histórica, o estado EPR é referente a um squeezing de dois modos de amplitude.
} 
autovalores de $V+i \Omega$ são positivos.

\subsubsection{Autovalores Simpléticos e Teorema de Williamson}

Supondo que haja uma transformação simplética, $S$, que permita calcular de forma simples os autovalores de $S V S^{T}+i \Omega$, o sinal dos mesmos irá determinar se o estado original é físico. A existência desta transformação é fornecida pelo Teorema de Williamson [119, 117]. Ele garante que, para qualquer matriz real, simétrica, positiva e de dimensão par, exista uma transformação simplética que a mapeia em uma matriz diagonal, positiva e duplamente degenerada em todos os seus elementos,

$$
S V S^{T}=V_{D}, \quad \text { onde } \quad V_{D}=D \oplus D \quad \text { e } \quad D=\operatorname{diag}\left(v_{1}, \ldots, v_{i}, \ldots, v_{n}\right){ }^{15}
$$

O símbolo $\oplus$ representa uma soma direta de matrizes e $v_{i}$ são os chamados autovalores simpléticos. A positividade dos autovalores de $V^{\prime}+i \Omega$ corresponde a $v_{i} \geq 1$ e, como isto deve ser válido para todos os autovalores simpléticos,

$$
v_{-} \geq 1
$$

é equivalente a (5.16), onde $v_{-}$é o menor autovalor simplético.

A transformação simplética de que trata o Teorema de Williamson não é uma transformação de similaridade, $S^{T} \neq S^{-1}$, pois ela não conserva os autovalores, apesar de conservar o determinante. Por este motivo, os autovalores simpléticos não são autovalores no sentido usual do termo. Eles são calculados a partir da diagonalização da matriz $M=(V \Omega)^{2}$, pois, como $S V \Omega S^{-1}=(D \oplus D) \Omega \Rightarrow S(V \Omega)^{2} S^{-1}=-D^{2} \oplus D^{2}$, a mesma transformação responsável pela a diagonalização simplética em $V$ realiza uma diagonalização em $M$.

Como discutido anteriormente, as transformações simpléticas em sistemas quânticos são equivalentes a evolução unitária devida a hamiltonianos biquadrados. Neste contexto, o Teorema de Williamson nos diz que há uma transformação unitária gaussiana que diagonaliza qualquer matriz de covariâncias na forma (5.27). Esta é a matriz de covariâncias de um estado térmico, com operador densidade dado por

$$
\hat{\rho}=\frac{\mathrm{e}^{-\beta \sum_{i=1}^{n} \hat{a}_{i}^{\dagger} \hat{a}_{i}}}{Z}, \quad \text { onde } \quad Z=\operatorname{Tr}\left[\prod_{i=1}^{n} \mathrm{e}^{-\beta \hat{a}_{i}^{\dagger} \hat{a}_{i}}\right]
$$

\footnotetext{
${ }^{15} \mathrm{~A}$ matriz $V_{D}$ é única a menos de permutações. Ela é apresentada mais comumente como $V_{D}=$ $\operatorname{diag}\left(v_{1}, v_{1}, \ldots, v_{n}, v_{n}\right)$.
} 
é a função de partição e $\beta=1 / K_{B} T$ é o fator de Boltzmann [120, 121]. Neste sistema, os autovalores simpléticos possuem uma interpretação física bem interessante, pois eles são iguais aos ruídos das quadraturas, $v_{i}=\Delta^{2} \hat{q}_{i}=\Delta^{2} \hat{p}_{i}=2 \bar{n}_{i}+1$, onde $\bar{n}_{i}$ é o número médio de fótons em cada modo. O Teorema de Williamson garante que a matriz de covariâncias de um estado físico qualquer possa ser convertida na de um estado térmico, e vice-versa, usando somente transformações unitárias. Em outras palavras, o estado original ${ }^{16}$ pode ser entendido como a evolução de um estado térmico sujeito a uma dinâmica unitária. Sendo assim, qualquer impureza presente em um estado gaussiano pode ser considerada como fruto do estado térmico inicial 17 .

Para testar o emaranhamento usando os autovalores simpléticos, basta substituir a matriz $V$ por $\tilde{V}=\Lambda V \Lambda$ no cálculo dos mesmos. Desta forma

$$
\tilde{v}_{-}^{k}<1
$$

se torna o critério de emaranhamento para a bipartição $h^{18}$ Note que, assim como a negatividade do operador densidade, esta é a expressão da não-fisicalidade após a transposição parcial. Esta diagonalização simplética é bastante prática, pois basta o conhecimento da matriz de covariâncias para a sua aplicação e os autovalores simpléticos podem ser calculados de forma eficiente para um sistema com um número arbitrário de modos. Note que como a relação (5.30) é derivada do critério PPT ela é uma condição suficiente para o emaranhamento. O que significa que se $\tilde{v}_{-}^{k}<1$, o estado original é certamente emaranhado.

\subsubsection{Sistemas de Dois Modos}

O critério PPT como mostrado em (5.30) é operacional, pois há uma receita de como aplicá-lo, mesmo que o cálculo dos autovalores simpléticos seja feito numericamente. Para sistemas de dois modos, entretanto, há uma expressão analítica fechada para $\tilde{v}_{-}$ e há ainda uma outra forma equivalente para o critério PPT baseada no determinante de $V+i \Omega$. Como para nossos propósitos esta segunda forma é mais interessante, vou

\footnotetext{
${ }^{16}$ desde que o mesmo seja gaussiano.

${ }^{17}$ Esta afirmação, entretanto, não é verdadeira no contexto experimental, pois o estado inicial em grande parte dos experimentos em ótica quântica é o estado de vácuo. Nestes sistemas, a dinâmica não é unitária e isto dará origem a impurezas. Porém, o Teorema de Williamson nos diz que este tipo evolução é matematicamente equivalente à evolução unitária partindo de um estado térmico.

${ }^{18}$ Por exemplo, um sistema tripartite tem 3 bipartições possíveis: $0 \otimes 12(k=0), 1 \otimes 02(k=1), 2 \otimes 01$ $(k=2)$.
} 
discutí-la a seguir. A matriz de covariâncias de um sistema de dois modos é da forma

$$
\begin{gathered}
V=\left[\begin{array}{cc}
A_{1} & C \\
C^{T} & A_{2}
\end{array}\right], \text { onde } \\
A_{i}=\left[\begin{array}{cc}
\left\langle\delta \hat{q}_{i}^{2}\right\rangle & \left\langle\delta \hat{q}_{i} \delta \hat{p}_{i}\right\rangle_{S} \\
\left\langle\delta \hat{q}_{i} \delta \hat{p}_{i}\right\rangle_{S} & \left\langle\delta \hat{p}_{i}^{2}\right\rangle
\end{array}\right], \quad C=\left[\begin{array}{cc}
\left\langle\delta \hat{q}_{1} \delta \hat{q}_{2}\right\rangle & \left\langle\delta \hat{q}_{1} \delta \hat{p}_{2}\right\rangle \\
\left\langle\delta \hat{q}_{2} \delta \hat{p}_{1}\right\rangle & \left\langle\delta \hat{p}_{1} \delta \hat{p}_{2}\right\rangle
\end{array}\right]
\end{gathered}
$$

$\operatorname{com}\left\langle\delta \hat{q}_{i} \delta \hat{p}_{i}\right\rangle_{S}=\left\langle\left\{\delta \hat{q}_{i}, \delta \hat{p}_{i}\right\}\right\rangle / 2$. As submatrizes $A_{1}$ e $A_{2}$ são as matrizes de covariâncias dos sistemas reduzidos e $C$ é uma matriz contento as correlações entre campos distintos, que são responsáveis pelo emaranhamento. Como o emaranhamento deve ser invariante por transformações unitárias locais, é possível converter a matriz de covariâncias para formas mais simples, chamadas formas padrão [118, 122, 123], sem alterar o emaranhamento. Para isto, usaremos as duas transformações simpléticas locais: evolução livre - que é uma rotação no espaço de fase - e squeezing local. A rotação no espaço de fase pode diagonalizar as matrizes $A_{1}$ e $A_{2}$, pois ambas são simétricas, e as operações de squeezing locais são usadas para balancear os ruídos de $q$ e $p$,

$$
A_{i}^{\prime}=S_{r o t}\left(\theta_{i}\right) A_{i} S_{r o t}^{T}\left(\theta_{i}\right)=\left(\begin{array}{cc}
a_{i} & 0 \\
0 & b_{i}
\end{array}\right) \quad \text { e } \quad A_{i}^{\prime \prime}=S_{s q}\left(\lambda_{i}\right) A_{i}^{\prime} S_{s q}^{T}\left(\lambda_{i}\right)=\alpha_{i}\left(\begin{array}{cc}
1 & 0 \\
0 & 1
\end{array}\right) .
$$

Os parâmetros $\theta_{i}$ e $\lambda_{i}$ dependem das entradas originais de $A_{1}$ e $A_{2}$ e os seus valores não são importantes neste contexto. Durante estas transformações, a matriz $C$ também será transformada em uma matriz $C^{\prime \prime}$ com entradas dependentes das originais e de $\theta_{i}$ e $\lambda_{i}$. Ela pode ser diagonalizada por duas rotações simultâneas nos modos 1 e 2,

$$
C^{\prime \prime} \Rightarrow S_{r o t}\left(\tilde{\theta}_{1}\right) C^{\prime \prime} S_{r o t}^{T}\left(\tilde{\theta}_{2}\right)
$$

escolhendo-se $\tilde{\theta}_{1}$ e $\tilde{\theta}_{2}$ adequadamente, o que pode ser verificado diretamente. Como as matrizes $A_{i}^{\prime \prime}$ são proporcionais à matriz identidade, elas não são afetadas por esta última transformação. Desta forma, é possível converter qualquer matriz de covariâncias para

$$
V_{f p 1}=\left(\begin{array}{cc}
A_{1}^{f p 1} & C^{f p 1} \\
\left(C^{f p 1}\right)^{T} & A_{2}^{f p 1}
\end{array}\right), \quad \text { onde } \quad A_{i}^{f p 1}=\alpha_{i} \mathcal{I} \quad \text { e } \quad C^{f p 1}=\left(\begin{array}{cc}
c_{q} & 0 \\
0 & c_{p}
\end{array}\right),
$$

que é chamada forma padrão 1. Note que dos 10 parâmetros que formam $V$, apenas 4 são importantes para o emaranhamento em estados gaussianos $-\alpha_{1}, \alpha_{2}, c_{q}$ e $c_{p}$. A transformação para a forma padrão é realizada utilizando-se somente operações simpléticas locais. Por este motivo, algumas quantidades são conservadas e são chamadas de invariantes simpléticos locais. As primeiras delas são as purezas locais, que são 
função do determinante das matrizes $A_{i}$, e do determinante da matriz $C$, $\operatorname{det}\left(A_{i}^{f p 1}\right)=\operatorname{det}\left(S_{i} A_{i} S_{i}^{T}\right)=\operatorname{det}\left(A_{i}\right) \quad \mathrm{e} \quad \operatorname{det}\left(C^{f p 1}\right)=\operatorname{det}\left(S_{1} C S_{2}^{T}\right)=\operatorname{det}(C), \operatorname{pois} \operatorname{det}\left(S_{i}\right)=1$ e onde $S_{1}$ e $S_{2}$ são transformações simpléticas que atuam somente nos modos 1 e 2 respectivamente. A pureza global também é conservada ${ }^{19}$ e o determinante da matriz $V^{f p 1}$ é

$$
\begin{aligned}
\operatorname{det}\left(V^{f p 1}\right)=\operatorname{det}(V)= & \alpha_{1}^{2} \alpha_{2}^{2}+\left(c_{q} c_{p}\right)^{2}-\alpha_{1} \alpha_{2}\left(c_{q}^{2}+c_{p}^{2}\right) \\
= & \operatorname{det}\left(A_{1}\right) \operatorname{det}\left(A_{2}\right)+\operatorname{det}(C)^{2}-I_{4}, \\
\operatorname{com} \quad & I_{4}=\alpha_{1} \alpha_{2}\left(c_{q}^{2}+c_{p}^{2}\right)=\operatorname{tr}\left(A_{1} J C J A_{2} J C^{T} J\right) .
\end{aligned}
$$

onde $J$ é definido em (5.18). Como pode ser verificado diretamente, $I_{4}$ também é invariante por transformações simpléticas locais e, junto com $I_{1}=\operatorname{det}\left(A_{1}\right), I_{2}=\operatorname{det}\left(A_{2}\right)$ e $I_{3}=\operatorname{det}(C)$, faz parte dos 4 invariantes simpléticos locais. Note que o número de parâmetros necessários para caracterizar o emaranhamento e a quantidade de invariantes locais é a mesma. Isto não é coincidência, pois o emaranhamento é uma propriedade, por definição, invariante por transformações unitárias locais. No caso de estados gaussianos, esta invariância se restringe às transformações simpléticas.

O determinante da matriz $V+i \Omega$ é positivo quando $V$ é a matriz de covariâncias de um estado físico, pois todos os seus autovalores são positivos. A matriz $V+i \Omega$ somente pode ter um autovalor negativo, portanto a positividade do seu determinante é equivalente à dos autovalores. Para mostrar isto, considere o determinante de $V+i \Omega$ na forma padrão 1 ,

$$
\operatorname{det}(V+i \Omega)=\alpha_{1}^{2} \alpha_{2}^{2}-\alpha_{1}^{2}-\alpha_{2}^{2}-\alpha_{1} \alpha_{2}\left(c_{p}^{2}+c_{q}^{2}\right)+\left(1-c_{p} c_{q}\right)^{2}
$$

A equação secular, $\operatorname{det}(V+i \Omega-\lambda)=0$, é equivalente $\operatorname{adet}(V+i \Omega)=0$, mas $\operatorname{com} \alpha_{i} \rightarrow$ $\alpha_{i}-\lambda$. Em função de $\lambda_{0}=\lambda-\left(\alpha_{1}+\alpha_{2}\right) / 2$,

$$
\left(\alpha_{1}-\lambda\right)\left(\alpha_{2}-\lambda\right)=\lambda_{0}^{2}-\frac{\left(\alpha_{1}-\alpha_{2}\right)^{2}}{4}, \quad\left(\alpha_{1}-\lambda\right)^{2}+\left(\alpha_{2}-\lambda\right)^{2}=2 \lambda_{0}^{2}+\frac{\left(\alpha_{1}-\alpha_{2}\right)^{2}}{2} .
$$

Portanto, a equação secular se torna uma equação do tipo $x^{2}+b x+c=0 \operatorname{com} x=\lambda_{0}^{2}$,

$$
b=-\frac{\left(\alpha_{1}-\alpha_{2}\right)^{2}}{2}-\left(c_{p}^{2}+c_{q}^{2}+2\right) \quad \text { e } \quad c=\frac{\left(\alpha_{1}-\alpha_{2}\right)^{2}}{4}\left(c_{p}^{2}+c_{q}^{2}-2\right)+\left(1-c_{p} c_{q}\right)^{2}+\frac{\left(\alpha_{1}-\alpha_{2}\right)^{4}}{16} .
$$

\footnotetext{
${ }^{19}$ Inclusive, ela é conservada por qualquer transformação simplética mesmo que global.
} 
Como a matriz $V+i \Omega$ é hermitiana, seus autovalores são reais e iguais a

$$
\lambda_{1 \pm}=\frac{\alpha_{1}+\alpha_{2}}{2} \pm \sqrt{x_{+}} \quad \text { e } \quad \lambda_{2 \pm}=\frac{\alpha_{1}+\alpha_{2}}{2} \pm \sqrt{x_{-}}
$$

onde $x_{ \pm}$são as raízes do polinômio em $x$. Também devido à hermiticidade de $V+i \Omega$, $c \geq 0$ e $x_{ \pm} \geq 0$. Como $\alpha_{i} \geq 0$, os dois autovalores $\lambda_{1+}$ e $\lambda_{2+}$ são sempre positivos. Para mostrar que o maior dentre $\lambda_{1-}$ e $\lambda_{2-}$ também deve ser positivo, usaremos

$$
x_{ \pm}=\frac{\left(\alpha_{1}-\alpha_{2}\right)^{2}}{4}+\frac{\left(c_{p}^{2}+c_{q}^{2}+2\right)}{2} \pm \frac{\sqrt{\cdots}}{2}
$$

Se supomos que máx $\left\{\lambda_{1-}, \lambda_{2-}\right\}<0$, então $\left(\alpha_{1}+\alpha_{2}\right)^{2} / 4-\min \left\{x_{+}, x_{-}\right\}<0$. Porém, como $\min \left\{x_{+}, x_{-}\right\}=x_{-}$,

$$
\left(\alpha_{1}+\alpha_{2}\right)^{2} / 4-\min \left\{x_{+}, x_{-}\right\}=\frac{\left(2 \alpha_{1} \alpha_{2}-c_{q}^{2}-c_{p}^{2}\right)}{2}+1+\frac{\sqrt{\cdots}}{2}>0,
$$

o que contradiz a hipótese inicial. A última desigualdade em (5.40) segue direto da desigualdade de Cauchy-Schwartz, pois $c_{q} \leq \sqrt{\alpha_{1} \alpha_{2}}$ e $c_{p} \leq \sqrt{\alpha_{1} \alpha_{2}}$. Portanto, o sinal do determinante de $V+i \Omega$ se torna o critério para que $V$ possa ser a matriz de covariâncias de um estado físico. A expressão (5.39) em função dos invariantes simpléticos se torna

$$
W_{\text {phys }} \equiv \operatorname{det}(V+i \Omega)=\operatorname{det}(V)+1-2 \operatorname{det}(C)-\sum_{i=1,2} \operatorname{det}\left(A_{i}\right) \geq 0 \text {, }
$$

A transposição parcial equivale à reversão temporal em um dos modos e, como $\tilde{V}=\Lambda V \Lambda$ em que $\Lambda=\operatorname{diag}(1,1,1,-1)$, apenas o sinal $\operatorname{de} \operatorname{det}(C)$ será modificado. Deste modo, a desigualdade

$$
W_{p p t}=\operatorname{det}(V)+1+2 \operatorname{det}(C)-\sum_{i=1,2} \operatorname{det}\left(A_{i}\right)<0
$$

se torna o critério PPT para sistemas bipartites formados por dois modos. Todo estado físico deve satisfazer a relação (5.41) e todo estado físico separável deve violar (5.42). Consequentemente, todo estado que satisfaz ambas as desigualdades (5.41) e (5.42) é emaranhado. Em outras palavras, (5.41) e (5.42) são uma condição suficiente para o emaranhamento em sistemas bipartites de dois modos. Se o estado for gaussiano, é possível deduzir também uma condição necessária para o emaranhamento [107]: todo estado emaranhado gaussiano possui $\operatorname{det}(C)<0$. A partir deste resultado, podemos mostrar que as desigualdades (5.41) e (5.42) são também uma condição necessária para o emaranhamento em sistemas bipartites de dois modos gaussianos [107]. 
O argumento para se chegar a esta última conclusão é discutido neste parágrafo e, apesar de interessante, não é essencial para a compreensão do restante do texto. Primeiro considere um estado gaussiano e emaranhado inicial. Ele possui $\operatorname{det}(C)<0$, pois é emaranhado, e $W_{p h y s} \geq 0$, pois é um estado físico. Há somente duas situações possíveis para $W_{p p t}$ : uma com $W_{p p t} \geq 0$ e outra com $W_{p p t}<0$. Na primeira delas, obtemos em um novo estado, $\rho^{\prime}$, após a transposição parcial que também é físico, pois $W_{\text {phys }}^{\prime}=W_{p p t} \geq 0$, mas é separável, pois $\operatorname{det}\left(C^{\prime}\right)=-\operatorname{det}(C) \geq 0$. Uma nova transposição parcial no estado $\rho^{\prime}$ necessariamente irá resultar em um outro estado físico e separável. Neste ponto chegamos à uma contradição, pois duas transposições parciais aplicadas no mesmo subsistema tem como resultado o próprio estado original que, por hipótese, é emaranhado. Como a primeira situação não é possível, somente nos resta a segunda, em que $\operatorname{det}(C)<0, W_{\text {phys }} \geq 0$ e $W_{p p t}<0$. Neste segundo caso, a transposição parcial sempre mapeia um estado emaranhado em um estado não-físico. Consequentemente, uma segunda transposição no mesmo subsistema irá mapear este estado não-físico de volta no estado emaranhado origina 20 Sendo assim, o critério PPT fornece uma fronteira clara entre o conjunto de estados gaussianos separáveis e emaranhados que é dada pela equação $W_{p p t}=0$, de tal modo que os estados desta fronteira são separáveis.

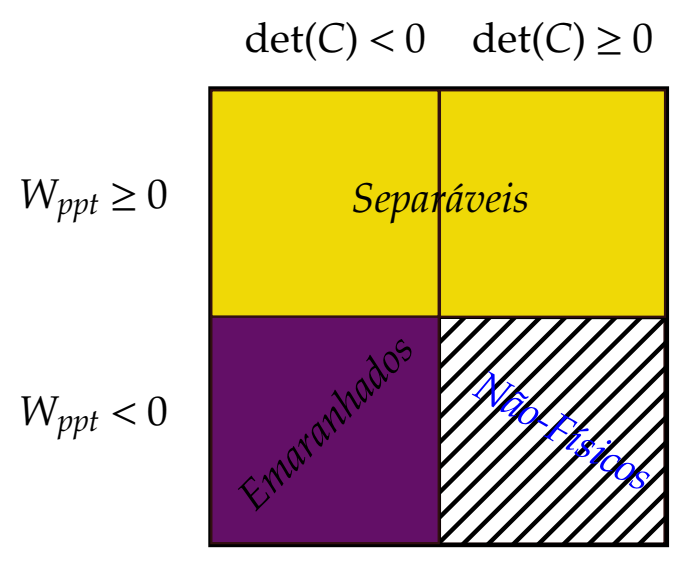

Figura 5.3: Figura esquemática comparando os critérios de emaranhamento $W_{p p t} e \operatorname{det}(C)$.

A condição (5.42) é equivalente a (5.30), fazendo com que esta também seja necessária e suficiente para o emaranhamento. Considerando o sistema geral (5.31) na forma padrão 1, o menor autovalor simplético após a transposição parcial é

$$
\tilde{v}_{-}=\frac{\Delta}{2}-\sqrt{\Delta^{2}-4 \operatorname{det}(V)},
$$

\footnotetext{
${ }^{20}$ Apesar de isto parecer estranho, não há problema, pois a transposição parcial não é uma transformação física e, consequentemente pode mapear estados físicos em estados não-físicos e viceversa.
} 
onde $\Delta=\operatorname{det}\left(A_{1}\right)+\operatorname{det}\left(A_{2}\right)-2 \operatorname{det}(C)$. Se escrevemos (5.42) $\operatorname{como} \operatorname{det}(V)+1<\Delta$, é possível mostrar que $\tilde{v}_{-}<1 \Leftrightarrow W_{p p t}<0$. Na próxima seção serão mostrados resultados originais utilizando o critério PPT na forma apresentada em (5.42) para estudar a dinâmica do emaranhamento sujeito a perdas neste tipo de sistema [27, 96].

\subsection{Análise da Robustez do Emaranhamento em Sistemas de Variáveis Contínuas de Dois Modos}

Estudamos o efeito da dissipação no emaranhamento em sistema de variáveis contínuas contendo um modo em cada parte. É bem conhecido o fato de que o emaranhamento neste tipo de sistema é degradado por perdas nos canais de transmissão [89]. Uma análise experimental de como ocorre essa degradação foi reportada em [124], mostrando que os estados que violavam o critério DGCZ são robustos frente a perdas. Neste trabalho, entretanto, são utilizados estados emaranhados criados a partir da interferência de estados comprimidos em divisores de feixes, procedimento que limita a geração de estados emaranhados entre luz de mesma frequência. Como discutirei na seção 5.4.2, estados criados desta maneira são sempre robustos frente a perdas.

As medidas de desemaranhamento frente a perdas serão apresentados no capítulo 7] e constituem a primeira observação de estados gaussianos de dois modos cujo emaranhamento desaparece por completo quando sujeitos a perdas. Discutirei aqui o estudo teórico que realizamos com o intuito de entender o que causa e, consequentemente, como evitar os estados que apresentam emaranhamento frágil frente a perdas. A partir deste estudo teórico, derivamos uma condição suficiente para que o emaranhamento seja robusto frente a perdas. Esta condição se torna também necessária para estados gaussianos.

\subsubsection{Critério PPT após Atenuação}

Vamos utilizar o critério PPT para estudar a dinâmica frente a perdas do emaranhamento em estados gaussianos, pois ele é uma condição necessária e suficiente para o emaranhamento nesta classe de estados. Modelamos a dissipação como perdas por uma das portas de um divisor de feixes, como mostrado em (2.17). Elas vão agir nos 
ruídos e correlações como

$$
\begin{aligned}
\Delta^{2} \hat{\xi}_{a}\left(T_{i}\right) & =\left\langle\left[\delta \hat{\xi}_{a}\left(T_{i}\right)\right]^{2}\right\rangle=\left\langle\left(\sqrt{T_{i}} \delta \hat{\xi}_{b} \pm \sqrt{1-T_{i}} \delta \hat{\xi}_{0 a}\right)^{2}\right\rangle=T_{i}\left(\Delta^{2} \hat{\xi}_{a}-1\right)+1 \\
\left\langle\hat{\xi}_{a}\left(T_{i}\right) \hat{\xi}_{b}\left(T_{j}\right)\right\rangle & =\left\langle\left(\sqrt{T_{i}} \delta \hat{\xi}_{a} \pm \sqrt{1-T_{i}} \delta \hat{\xi}_{0 a}\right)\left(\sqrt{T_{j}} \delta \hat{\xi}_{b} \pm \sqrt{1-T_{j}} \delta \hat{\xi}_{0 b}\right)\right\rangle=\sqrt{T_{i} T_{j}}\left\langle\hat{\xi}_{a} \hat{\xi}_{b}\right\rangle,
\end{aligned}
$$

pois $\hat{\xi}_{a}$ e $\hat{\xi}_{b 0}$ são descorrelacionados. Estas expressões são compactadas em função da matriz de covariâncias como

$$
V \rightarrow V^{\prime}=\mathcal{L}(V)=L(V-\mathcal{I}) L+\mathcal{I}
$$

onde $\mathcal{I}$ é a matriz identidade, $L=\operatorname{diag}\left(\sqrt{T_{1}}, \sqrt{T_{1}}, \sqrt{T_{2}}, \sqrt{T_{2}}\right)$ e $T_{i}$ são os coeficientes de transmissão. A transformação (5.44) se traduz nas matrizes $A_{1}, A_{2}$ e $C$ como

$$
A_{i}^{\prime}=T_{i} A_{i}+\left(1-T_{i}\right) \mathcal{I} \quad \text { e } \quad C^{\prime}=\sqrt{T_{1} T_{2}} C .
$$

Como o critério PPT depende apenas do sinal de $W_{p p t} t^{21}$, se as atenuações se fatorassem para todas as quantidades envolvidas em (5.42) de forma simples como ocorre com $\operatorname{det}(C)$, o estado se manteria emaranhado até atenuação total. Entretanto, devido a (5.44) e a primeira equação de (5.45), essa fatoração não ocorre para as purezas global e locais. Estas possuem uma dinâmica não-linear em função das perdas, chegando a $\mu=\mu_{i}=1$ para atenuação total. Esta dinâmica não-linear das purezas é a origem dos estados de emaranhamento frágil.

O procedimento que adotaremos será calcular $W_{p p t}$ após a atenuação,

$$
W_{p p t}^{\prime}\left(T_{1}, T_{2}\right)=\operatorname{det}\left(V^{\prime}\right)+1+2 \operatorname{det}\left(C^{\prime}\right)-\sum_{j=1,2} \operatorname{det}\left(A_{i}^{\prime}\right) \text {. }
$$

Um ponto muito importante a ressaltar é de que aqui não podemos usar a forma padrão 1 como fizemos antes porque a operação que leva à forma padrão 1 - mais especificamente a transformação de squeezing - não comuta com a atenuação. Portanto, se aplicarmos a atenuação após passar para a forma padrão 1, a matriz de covariâncias obtida não necessariamente é a mesma que obteríamos se invertêssemos os processos. Como no experimento a atenuação é aplicada no sistema real, antes de passar para a forma padrão 1, o mesmo deve ser feito aqui.

O emaranhamento do sistema é dito robusto quando $W_{p p t}^{\prime}\left(T_{1}, T_{2}\right)<0$ dentro de toda a região em que $0<T_{1}, T_{2}<1$. Para calcular a dependência explícita com relação à

\footnotetext{
${ }^{21}$ A partir deste ponto, vou supor que $W_{\text {phys }} \geq 0$ sempre. Esta suposição é completamente razoável e justificada, principalmente no contexto experimental, pois qualquer estado que possamos preparar é, por definição, físico.
} 
atenuação, vamos reescrever cada um dos termos de (5.46) em função de quantidades que escalam de forma simples com as perdas. As mais diretas delas são

$$
\operatorname{det}\left(C^{\prime}\right)=T_{1} T_{2} \operatorname{det}(C) \quad \text { e } \quad \operatorname{det}\left(V^{\prime}-\mathcal{I}\right)=T_{1}^{2} T_{2}^{2} \operatorname{det}(V-\mathcal{I})
$$

Como qualquer matriz $2 \times 2$ possui as propriedades

$$
\operatorname{det}(M-\mathcal{I})=\operatorname{det}(M)-\operatorname{tr}(M)+1 \quad \text { e } \operatorname{tr}(M-\mathcal{I})=\operatorname{tr}(M)-2,
$$

a atenuação também é fatorada nas quantidades

$$
\gamma_{i}^{\prime}-\delta_{i}^{\prime}=T_{i}^{2}\left(\gamma_{i}-\delta_{i}\right) \quad \text { e } \quad \delta_{i}^{\prime}=T_{i} \delta_{i}
$$

onde $\gamma_{i}=\operatorname{det}\left(A_{i}\right)-1$ e $\delta_{i}=\operatorname{tr}\left(A_{i}\right)-2$, tal que $\gamma_{i}-\delta_{i}=\operatorname{det}\left(A_{i}-\mathcal{I}\right)$. $\gamma_{i}$ é somente uma maneira conveniente de se escrever as purezas dos subsistemas. Ela é nula para um estado puro, positiva para uma mistura estatística e tende a infinito quanto mais o estado estiver longe de um estado puro. A quantidade $\delta_{i}$ é a soma dos ruídos de $\hat{p}$ e $\hat{q}$ de cada subsistema menos os ruídos de $\hat{p}$ e $\hat{q}$ de um estado de vácuo. Por este motivo, ela será chamada de excesso de ruído no decorrer deste texto. Este excesso de ruído é nulo para um estado coerente e positivo para qualquer outro estado físico. A pureza global pode ser escrita em função de $\delta_{i}$ e $\gamma_{i}$ como

$$
\begin{gathered}
\operatorname{det}(V)=\operatorname{det}(V-\mathcal{I})+\eta, \quad \text { onde } \\
\eta=\delta_{1}\left(\gamma_{2}-\delta_{2}\right)+\delta_{2}\left(\gamma_{1}-\delta_{1}\right)+\operatorname{det}\left(A_{1}\right)+\operatorname{det}\left(A_{2}\right)+\delta_{1} \delta_{2}+\operatorname{Tr}_{1}+\operatorname{Tr}_{2}-\operatorname{tr}\left(C^{T} C\right)-1, \\
\operatorname{Tr}_{1}=\operatorname{tr}\left(C^{T} J\left(A_{1}-\mathcal{I}\right) J C\right) \quad \text { e } \operatorname{Tr}_{2}=\operatorname{tr}\left(C J\left(A_{2}-\mathcal{I}\right) J C^{T}\right)
\end{gathered}
$$

Estas últimas quantidades escalam com as perdas como

$$
\operatorname{Tr}_{1}^{\prime}=T_{1}^{2} T_{2} \operatorname{Tr}_{1}, \quad \operatorname{Tr}_{2}^{\prime}=T_{1} T_{2}^{2} \operatorname{Tr}_{2}, \quad \text { e } \operatorname{tr}\left(C^{T} C\right)=T_{1} T_{2} \operatorname{tr}\left(C^{T} C\right)
$$

Quando substituímos (5.50) em (5.20) e aplicamos a atenuação em todos os seus termos, chegamos em

$$
\begin{gathered}
\left.W_{p p t}^{\prime}\left(T_{1}, T_{2}\right)=T_{1} T_{2} W_{H}, \quad \operatorname{com} \quad W_{H}=T_{1} T_{2} \Gamma_{22}+T_{1} \Gamma_{21}+T_{2} \Gamma_{12}+\Gamma_{11}\right] \\
\Gamma_{22}=\operatorname{det}(V-\mathcal{I})=\operatorname{det}(V)-\eta, \quad \Gamma_{12}=\operatorname{Tr}_{2}+\delta_{1}\left(\gamma_{2}-\delta_{2}\right), \\
\Gamma_{21}=\operatorname{Tr}_{1}+\delta_{2}\left(\gamma_{1}-\delta_{1}\right), \quad \Gamma_{11}=\delta_{1} \delta_{2}-\operatorname{tr}\left(C^{T} C\right)+2 \operatorname{det}(C) .
\end{gathered}
$$

Como era de se esperar, quando qualquer um dos campos sofre atenuação total, o 
sistema é sempre separável, $W_{p p t}^{\prime}\left(T_{1}, 0\right)=W_{p p t}^{\prime}\left(0, T_{2}\right)=02$ Para qualquer $T_{1} \neq 0$ e $T_{2} \neq 0$, podemos fatorar (5.53) de modo que $W_{H}$ pode ser usada no lugar de $W_{p p t}^{\prime}$ como critério de emaranhamento, pois ambas guardam a mesma informação. Todas as possíveis dinâmicas de $W_{p p t}^{\prime}$ frente a perdas são mostradas na figura 5.4, para isso usamos uma matriz de covariâncias mais simples na forma
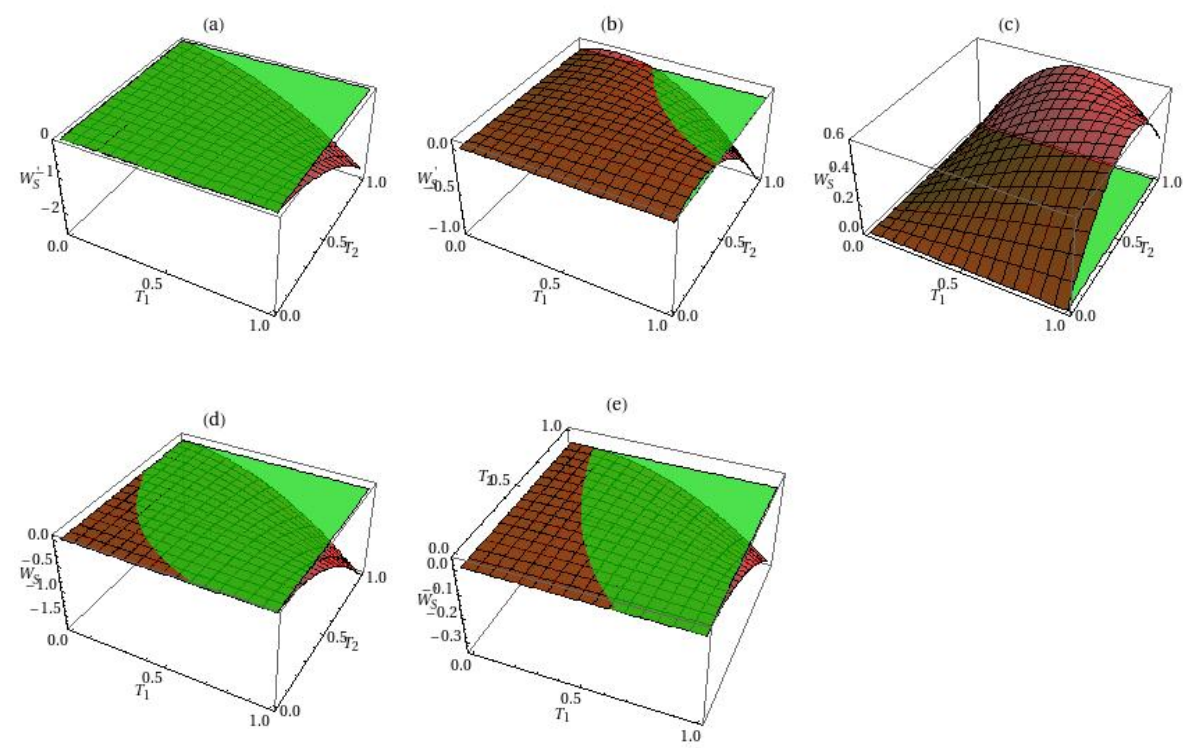

Figura 5.4: Curvas de $W_{p p t}^{\prime}\left(T_{1}, T_{2}\right)$. Os estados do tipo mostrado em $a$ ) são robustos. $\mathrm{O}$ emaranhamento presente neles somente é perdido para atenuação total em ambos os subsistemas. O estado $c$ ) é separável e mantendo-se sempre assim quando atenuado. Os estados $d$ ) e $e$ ) são parcialmente robustos com relação a um e dois subsistemas. E o estado $b$ ) se torna separável antes da atenuação total para qualquer um dos subsistemas.

$$
\left[\begin{array}{cccc}
\Delta^{2} q_{1} & 0 & c_{q} & 0 \\
0 & \Delta^{2} p_{1} & 0 & c_{p} \\
c_{q} & 0 & \Delta^{2} q_{2} & 0 \\
0 & c_{p} & 0 & \Delta^{2} p_{2}
\end{array}\right]
$$

As curvas a)-d) são construídas com os seguintes parâmetros comuns: $\Delta^{2} q_{1}=\Delta^{2} q_{2}=$ $2.55, \Delta^{2} p_{1}=\Delta^{2} p_{2}=1.80$ e $c_{p}=-1.26$. Passamos de uma situação para a outra variando o parâmetro $c_{q}$. A figura a) representa um estado emaranhado robusto, com $c_{q}=1.275$. $\mathrm{Na}$ figura b), o estado é frágil com relação a atenuação de qualquer uma das partes, com $c_{q}=0.893$. Um exemplo de estado separável é mostrado na figura c), onde $c_{q}=0.3825$. Escolhendo $c_{q}=1.03$, o sistema é frágil para atenuação conjunta nos dois subsistemas,

\footnotetext{
${ }^{22}$ Isto significa que o estado formado quando um dos campos está no estado de vácuo é sempre separável.
} 
mas é robusto quando atenuamos apenas uma das partes, como pode ser visto na figura d). Para construir a matriz de covariâncias cuja dinâmica é mostrada na figura e), uma das partes foi atenuada até que o estado gerado se torne frágil para atenuação em uma delas. Isto ocorre para a seguinte matriz de covariâncias,

$$
\left[\begin{array}{cccc}
2.55 & 0 & 0.653 & 0 \\
0 & 1.80 & 0 & -0.797 \\
0.653 & 0 & 1.62 & 0 \\
0 & -0.797 & 0 & 1.32
\end{array}\right]
$$

Os estados que são frágeis para atenuação em apenas uma das partes são chamados de parcialmente robustos. Por definição, todos os estados robustos são também parcialmente robustos.

A função $W_{H}$ é um parabolóide hiperbólico com coeficientes $\Gamma_{i j}$ e possui uma propriedade bastante útil para nossa análise: a curva definida por $W_{H}\left(T_{1}, T_{2}\right)=$ constante é uma hipérbole com assíntotas dadas por $T_{1}=$ constante e $T_{2}=$ constante. Quando $W_{H}\left(T_{1}, T_{2}\right)=0$, a curva formada é o conjunto de atenuações para as quais o estado emaranhado se torna separável. Ela divide os estados emaranhados dos separáveis dentro da região de atenuações físicas. A quantidade $W_{H}$ é mostrada na figura 5.5 e a fronteira consiste das atenuações que satisfazem $W_{H}\left(T_{1}, T_{2}\right)=0$. Devido ao fato de os coeficientes $\Gamma_{i j}$ serem dependentes entre si, a hipérbole $W_{H}\left(T_{1}, T_{2}\right)=0$ não pode ter uma orientação arbitrária. Cada ramo da mesma deve ser formado por uma função monotonicamente decrescente em relação a qualquer uma das atenuações, como mostrado na figura $5.5^{23}$.

\subsubsection{Critérios para a Robustez do Emaranhamento}

Todas as dinâmicas possíveis mostradas na figura 5.4 somente dependem do sinal de $W_{H}$ nos quatro vértices do quadrado verde mostrado em 5.5 . Estes vértices determinam a região de atenuações físicas em que $0 \leq T_{1}, T_{2} \leq 1$. Chamaremos $W_{H}$ calculada nestes pontos de

$$
W_{1}=W_{H}(1,0)=\Gamma_{12}+\Gamma_{11}, \quad W_{2}=W_{H}(0,1)=\Gamma_{21}+\Gamma_{11}, \quad W_{R E}=W_{H}(0,0)=\Gamma_{11}
$$

e $W_{p p t}=W_{H}(1,1)=\sum_{i, j} \Gamma_{i j}$. Por enquanto, vou restringir a discussão a estados gaussia-

\footnotetext{
${ }^{23} \mathrm{~A}$ equação $W_{H}\left(T_{1}, T_{2}\right)=0$ pode ser escrita como $T_{2}=f\left(T_{1}\right)$ ou $T_{1}=S\left(T_{2}\right)$. Em ambas as formas, devemos obter funções decrescentes [96].
} 

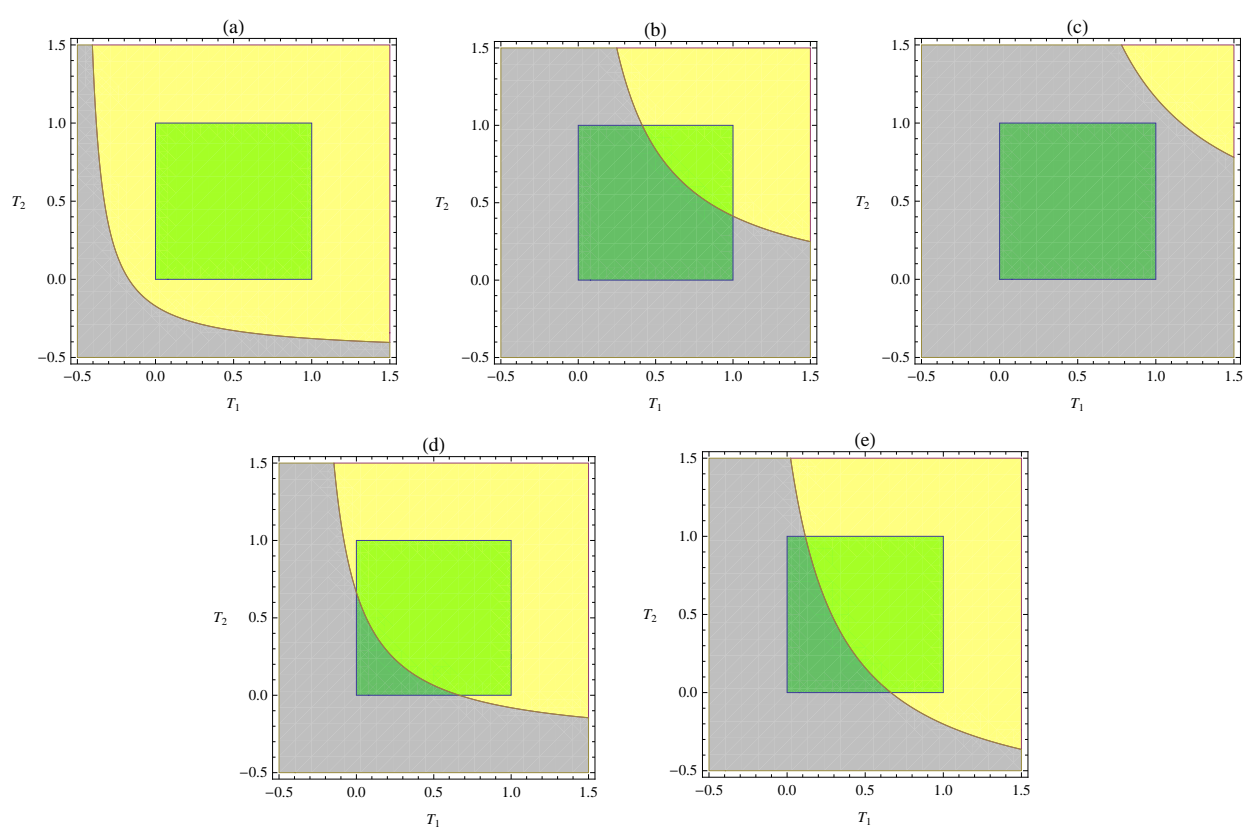

Figura 5.5: $W_{H}^{\prime}\left(T_{1}, T_{2}\right)$ calculada para as mesmas matrizes presentes na figura 5.4 . A região amarela é formada por estados emaranhados e a cinza contém os estados separáveis. O quadrado representa a região física, determinada por $0<T_{i} \leq 1$.

nos, pois para estes sistemas o critério PPT é necessário e suficiente. Neste caso, o sinal de $W_{p p t}$ determina o emaranhamento do estado inicial. Se $W_{p p t} \geq 0$, o estado inicial é separável e vai continuar separável para qualquer atenuação, pois estas são aplicadas de forma independente $e^{24}$ e o emaranhamento não pode ser criado por operações locais. Sendo assim, os casos de interesse ocorrem somente quando $W_{p p t}<0$. Como o emaranhamento não pode ser criado por perdas aplicadas de maneira independente, somente um estado inicialmente emaranhado pode estar emaranhado após as perdas. Em outras palavras, $W_{H}\left(T_{1}, T_{2}\right)<0$, calculado em qualquer $T_{1}$ ou $T_{2}$, é uma condição de emaranhamento para o estado original, porém menos restritiva que $W_{p p t}<0^{25}$. Bem próximo da atenuação total em ambos os subsistemas, $W_{H}$ se torna $W_{R E}$. Portanto, $W_{H}\left(T_{1}, T_{2}\right)<0$, para qualquer $T_{1}$ e $T_{2}$, sempre que $W_{R E}<0$. O que equivale a dizer que

$$
W_{R E}=\delta_{1} \delta_{2}-\operatorname{tr}\left(C^{T} C\right)+2 \operatorname{det}(C)<0
$$

é condição necessária e suficiente para que estados gaussianos de dois modos sejam robustos frente a perdas. Todos os elementos que compõem $W_{R E}$ são invariantes por rotação local nas quadraturas. Isto é razoável, pois a escolha da base de quadratura

\footnotetext{
${ }^{24}$ Consequentemente, são transformações locais.

${ }^{25} \mathrm{O}$ que quero dizer com isto é que enquanto todo estado para o qual $W_{H}\left(T_{1}, T_{2}\right)<0$ é emaranhado, existem alguns estados emaranhados para os quais $W_{H}\left(T_{1}, T_{2}\right) \geq 0$. Em outras palavras, a menos do ponto $T_{1}=T_{2}=1, W_{H}\left(T_{1}, T_{2}\right)<0$ é somente uma condição suficiente para o emaranhamento no estado original.
} 
na qual fazemos o experimento não deve mudar as propriedades físicas do estado. Sabendo disto, existe uma escolha de quadraturas na qual $C=\operatorname{diag}\left(c_{q}, c_{p}\right)$, e $W_{R E}$ se torna

$$
W_{R E}^{\mathrm{sb}}=\delta_{1} \delta_{2}-\left(c_{q}-c_{p}\right)^{2},
$$

pois $\delta_{1}$ e $\delta_{2}$ são invariantes por rotações. Consequentemente, todos os estados gaussianos de emaranhamento frágil satisfazem $\sqrt{\delta_{1} \delta_{2}}>\left|c_{q}\right|+\left|c_{p}\right|$ em alguma base de quadraturas, pois $\operatorname{det} C=c_{p} c_{q}<0$ para estados gaussianos emaranhados [107].

Os estados de emaranhamento frágil são caracterizados por um aumento dos excessos de ruído em relação ao valor absoluto das correlações. Quando $\delta_{1}=\delta_{2}$, a relação $\beta=\delta /\left(\left|c_{p}\right|+\left|c_{q}\right|\right)>1$ deve ser satisfeita para que os estados emaranhados sejam frágeis. Sendo assim, a forma mais direta de se construir estados frágeis a partir de estados robustos consiste em introduzir ruído em $\hat{p}$ e/ou $\hat{q}$, modificando pouco as correlações $c_{p}$ e $c_{q}$, até que $\beta$ se torne maior que 1. Esse ruído tipicamente diminui a pureza do estado global, o que sugere uma relação entre a robustez do emaranhamento e a pureza do sistema global. Esta relação não é válida de forma geral, pois estados puros também podem ser frágeis e podem ser construídos a partir de estados robustos por meio de squeezing local. Neste caso, o ruído de $\hat{p}$ aumenta por um fator $e^{2 r}$, onde $r>0$ é o fator de squeezing, enquanto o de $\hat{q}$ diminui pelo mesmo fator mantendo a multiplicação dos ruídos constantes. Entretanto, a soma dos mesmos sempre aumenta em relação à original. Como $c_{p}$ e $c_{q}$ serão multiplicadas por $e^{r}$ e $e^{-r}$, pode ocorrer que, para algum $r$, o desbalanceio entre o excesso de ruído e as correlações fará com que $\beta>1$.

A condição de simetria por rotação de $W_{R E}$ é menos restritiva que a satisfeita por $W_{p p t}$, pois esta deve ser invariante por qualquer transformação simplética local $\sqrt{26}$, Para um modo, estas transformações são somente duas: rotação no espaço das fase e squeezing local. Claramente, $W_{R E}$ é modificada por uma transformação de squeezing local $S_{\text {local }}=S\left(r_{1}\right) \oplus S\left(r_{2}\right)$, com $S(r)=\operatorname{diag}\left(e^{r}, e^{-r}\right)$. De fato, squeezings locais podem transformar qualquer estado frágil em robusto [96].

Como dito anteriormente, (5.57) é um critério suficiente de emaranhamento. Entretanto, os estados emaranhados que não satisfazem (5.57) se tornam separáveis após

\footnotetext{
${ }^{26}$ Transformações unitárias locais não podem mapear estados emaranhados em separáveis, mas podem mapear estados emaranhados que são detectados pelo critério $P P T$ em outros que não o são. $\mathrm{O}$ que corresponde a modificar o sinal de $W_{p p t}$. Consequentemente $W_{p p t}$ não é invariante por transformações unitárias gerais. Porém se estas transformações forem gaussianas, elas irão mapear estados gaussianos em outros estados gaussianos. Como o critério $P P T$ é necessário e suficiente para estes estados, $W_{p p t}$ deve se manter negativa após qualquer transformação unitária gaussiana local. O que somente é garantido se $W_{p p t}$ for invariante por transformações simpléticas locais, pois operações gaussianas aplicadas no operador densidade correspondem a transformações simpléticas aplicadas em $V$.
} 
perdas parciais A fronteira entre os estados frágeis e robustos no espaço de estados físicos 27 , definida por $W_{R E}=0$, é composta por estados emaranhados robustos ou separáveis, pois há alguns estados para os quais $W_{R E}=W_{p p t}=q^{28}$. Note que as perdas introduzidas na matriz de covariâncias, são linearmente fatoradas em $W_{R E}$,

$$
W_{R E}^{\prime}\left(T_{1}, T_{2}\right)=T_{1} T_{2}\left[\delta_{1} \delta_{2}-\operatorname{tr}\left(C^{T} C\right)+2 \operatorname{det}(C)\right]<0, \quad \text { sempre que } \quad W_{R E}<0
$$

De todos os estados mostrados na figura 5.4. apenas o exemplo a) é robusto. Uma caraterística importante que o diferencia dos outros exemplos apresentados é que ele sempre continuará emaranhando até atenuação total em ambas as partes independentemente de como a atenuação seja feita. Dentro dos estados frágeis, existem alguns que podem ser robustos quando atenuamos somente em uma das partes. Estes serão chamados de parcialmente robustos e são determinados pelos sinais de $W_{1}$ e $W_{2}$. A figura d) é um exemplo de estado de emaranhamento frágil para o qual $W_{1}<0$ e $W_{2}<0$. Apesar de se tornar separável para perdas introduzidas em ambos os subsistemas, o emaranhamento é robusto quando qualquer uma das partes é atenuada individualmente. O outro caso de robustez parcial ocorre quando o estado emaranhado é robusto ao se atenuar apenas um dos subsistemas, mas pode se tornar separável quando se atenua individualmente o outro subsistema. Eles são mostrados na figura e) e são definidos por $W_{1}<0$ e $W_{2} \geq 0$ ou $W_{2}<0$ e $W_{1} \geq 0$. Os estados do tipo b), possuem $W_{1} \geq 0$ e $W_{2} \geq 0$, e se tornam separáveis para perdas parciais quando qualquer uma das partes é atenuada. Estes estado possuem emaranhamento frágil para perdas introduzidas de qualquer maneira e são os piores estados para serem usados em protocolos de comunicação quântica no que diz respeito às perdas por transmissão.

Quando apenas uma das partes é atenuada, podemos calcular para qual transmissão o estado emaranhado se torna separável. Vamos considerar que apenas o subsistema 1 seja atenuado. Para isso considere $T_{2}=1 \mathrm{em}$ (5.53),

$$
W_{H}\left(T_{1}, 1\right)=T_{1}\left(\Gamma_{22}+\Gamma_{21}\right)+\Gamma_{12}+\Gamma_{11}
$$

Este estado é frágil em relação a perdas no subsistema 1 quando $W_{1}>0$. O coeficiente de atenuação para o qual o sistema se torna separável é

$$
T_{1}^{c}=\frac{W_{1}}{W_{1}-W_{p p t}}
$$

\footnotetext{
${ }^{27}$ Rigorosamente, estamos falando do espaço de matrizes de covariâncias. Porém para estados gaussianos, estes dois espaços são equivalentes.

${ }^{28}$ Alguns exemplos de estados com esta característica serão mostrados mais adiante.
} 
Como $W_{p p t}<0$, então $W_{1}-W_{p p t}>W_{1}>0$. Isto garante que $0<T_{1}^{c}<1$ sempre que $W_{p p t}<0$ e $W_{1}>0$. Uma condição semelhante ocorre para o subsistema 2 se substituímos $W_{1}$ por $W_{2}$ em (5.61). Na tabela 5.1 são mostradas as diversas situações de robustez do emaranhamento sempre supondo que o estado inicial viola o critério $P P T, W_{p p t}<0$.

Tabela 5.1: Condições para os diversos tipo de robustez

\begin{tabular}{cccc}
\hline \hline$W_{R E}$ & $W_{1}$ & $W_{2}$ & \\
\hline$\leq 0$ & $\leq 0$ & $\leq 0-$ & Robusto para qualquer tipo de atenuação \\
$>0$ & $\leq 0$ & $\leq 0-$ & Robusto para um modo/Frágil para os dois \\
$>0$ & $>0$ & $\leq 0-$ & Robusto para apenas um modo/Frágil para o outro modo \\
$>0$ & $\leq 0$ & $>0-$ & Robusto para apenas um modo/Frágil para o outro modo \\
$>0$ & $>0$ & $>0-$ & Frágil para qualquer um dos modos \\
\hline
\end{tabular}

A figura 5.6 mostra as regiões de estados separáveis e de emaranhamento frágil e robusto. Por simplicidade, a matriz de covariâncias utilizada é simétrica, com $\Delta^{2} q_{1}=$ $\Delta^{2} q_{2}=\Delta^{2} q$ e $\Delta^{2} p_{1}=\Delta^{2} p_{2}=\Delta^{2} p$. No gráfico consideramos $\Delta^{2} q$ e $\Delta^{2} p$ fixos e variando as correlações normalizadas $\bar{c}_{q}=c_{q} / \Delta^{2} q$ e $\bar{c}_{p}=c_{p} / \Delta^{2} p$

\subsubsection{Exemplos de Estados Simples}

Neste ponto, darei alguns exemplos de estados de emaranhamento robusto e frágil mais simples.

\section{Estado Completamente Simétrico}

O primeiro deles é o estado completamente simétrico, dado por

$$
V=\left(\begin{array}{cccc}
s & 0 & c & 0 \\
0 & s & 0 & -c \\
c & 0 & s & 0 \\
0 & -c & 0 & s
\end{array}\right),
$$

em que $s, c>0$. Exemplos deste tipo são os estados de squeezing de dois modos térmico. Estes são gerados quando aplicamos uma transformação de squeezing de dois modos,

$$
S_{E P R}(r)=e^{r\left[a_{1} a_{2}-a_{1}^{\dagger} a_{2}^{\dagger}\right]}
$$

em um estado térmico com número médio de fótons iguais em ambos os modos. $\mathrm{O}$ estado resultante, $\rho=U(r) \rho U(r)^{\dagger}$, possui uma matriz de covariâncias na forma 5.62 


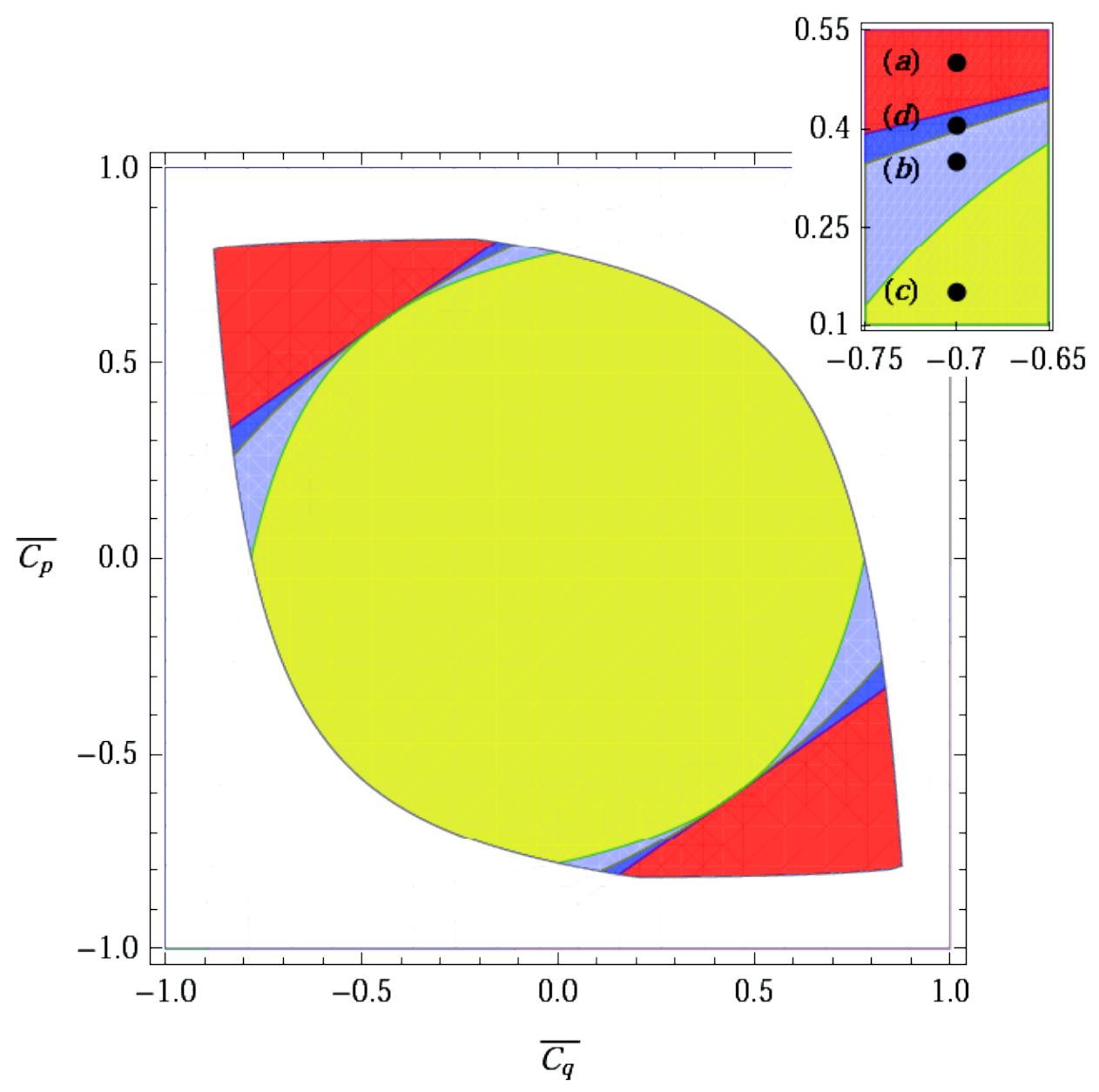

Figura 5.6: As regiões amarela e vermelha consistem dos estados separáveis e emaranhados robustos, respectivamente. A região azul representa os estados frágeis, sendo que os parcialmente robustos estão em azul escuro. A região branca corresponde a estados não-físicos. Os pontos destacados correspondem aos da figura 5.4. As quantidades $\bar{c}_{p} \mathrm{e} \bar{c}_{q}$ são as correlações normalizadas, definidas como $\bar{c}_{q}=c_{q} / \Delta^{2} q \mathrm{e} \bar{c}_{p}=c_{p} / \Delta^{2} p$

tal que $s=v \cosh (r)$ e $c=v \sinh (r)$, onde $v=2 \bar{n}+1$ e $\bar{n}$ é o número médio de fótons em cada modo. A pureza deste estado é $\mu=v^{-2}$, consequentemente, o estado somente é puro se $\bar{n}=0$, ou seja, se cada modo estiver no estado de vácuo. Para os estados como (5.62),

$$
\begin{gathered}
W_{p p t}=\left(s^{2}-c^{2}\right)^{2}+1-2 c^{2}-2 s^{2}=\left(s^{2}-c^{2}+1-2 s\right)\left(s^{2}-c^{2}+1+2 s\right) \\
\text { e } W_{R E}=4(s-1)^{2}-4 c^{2}=4\left(s^{2}-c^{2}+1-2 s\right) .
\end{gathered}
$$

Sabemos que $W_{p p t}<0$ sempre que $W_{R E}<0$, pois não podemos criar emaranhamento a partir de atenuações independentes. Como, neste exemplo, $W_{p p t}$ é proporcional a $W_{R E}$, o fator $s^{2}-c^{2}+1+2 s>0$ sempre que $W_{R E}<0$. Se supomos agora que $W_{p p t}<0$, um dos fatores da última igualdade em 5.64 dever ser negativo. Como $s>0, s^{2}-c^{2}+1+2 s>s^{2}-$ 
$c^{2}+1-2 s$ e concluímos que o fator negativo é o proporcional a $W_{R E}$. Consequentemente, $W_{R E}<0$ sempre que $W_{p p t}<0$. Em outras palavras, estados emaranhado com matrizes de covariâncias na forma 5.62 são sempre robustos, pois

$$
W_{R E}<0 \Leftrightarrow W_{p p t}<0
$$

No caso do squeezing de dois modos, para o qual $s=\cosh (r)$ e $c=\sinh (r)$,

$$
W_{p p t}=4(1-s)(1+s) \quad \text { e } \quad W_{R E}=8(1-s)
$$

Como $\cosh (r) \geq 1$, este estado é sempre emaranhado e robusto, $W_{p p t}<0$ e $W_{R E}<0$, se tornando separável somente quando $r=0$.

\section{Estado Simétrico}

Neste texto, chamo de sistemas simétricos aqueles que possuem as mesmas matrizes de covariâncias para os sistemas reduzidos, $A_{1}=A_{2}$. Vou supor também que $p$ e $q$ do mesmo subsistema sejam descorrelacionados. A matriz de covariâncias neste caso será

$$
V=\left(\begin{array}{cccc}
\Delta^{2} q & 0 & c_{q} & 0 \\
0 & \Delta^{2} p & 0 & c_{p} \\
c_{q} & 0 & \Delta^{2} q & 0 \\
0 & c_{p} & 0 & \Delta^{2} p
\end{array}\right) .
$$

Este exemplo é muito importante, pois os estados que são gerados no nosso OPO, e em grande parte dos experimentos envolvendo feixes gêmeos, possuem matrizes de covariâncias na forma 5.68. Neste caso, $W_{p p t}$ e $W_{R E}$ são

$$
\begin{gathered}
W_{p p t}=\left[\left(\Delta^{2} p\right)^{2}-c_{p}^{2}\right]\left[\left(\Delta^{2} q\right)^{2}-c_{q}^{2}\right]-2 \Delta^{2} p \Delta^{2} q+2 c_{p} c_{q}+1 \\
\text { e } \quad W_{R E}=\left(\Delta^{2} p+\Delta^{2} q-2\right)^{2}-\left(c_{q}-c_{p}\right)^{2}
\end{gathered}
$$

Este tipo de sistema pode ser analisado de forma mais simples passando para o espaço de soma e subtração, no qual os operadores canônicos se transformam em

$$
p_{ \pm}=\frac{1}{\sqrt{2}}\left(p_{1} \pm p_{2}\right) \quad \text { e } \quad q_{ \pm}=\frac{1}{\sqrt{2}}\left(q_{1} \pm q_{2}\right) .
$$

As entradas de $V$ são funções apenas dos ruídos no espaço de soma e subtração, $2 \Delta^{2} p=\Delta^{2} p_{+}+\Delta^{2} p_{-}$e $2 c_{p}=\Delta^{2} p_{+}-\Delta^{2} p_{-}$, tal que o mesmo vale para $q$. Em função destas 
novas variáveis,

$$
\begin{array}{cc}
W_{p p t}=W_{p} \bar{W}_{p} \text { e } & W_{R E}=W_{s} \bar{W}_{s}, \quad \text { onde } \\
W_{s}=\Delta^{2} \hat{p}_{+}+\Delta^{2} \hat{q}_{-}-2, & W_{p}=\Delta^{2} \hat{p}_{+} \Delta^{2} \hat{q}_{-}-1, \\
\bar{W}_{s}=\Delta^{2} \hat{p}_{-}+\Delta^{2} \hat{q}_{+}-2, & \bar{W}_{p}=\Delta^{2} \hat{p}_{-} \Delta^{2} \hat{q}_{+}-1 .
\end{array}
$$

Quando olhamos para a robustez parcial,

$$
W_{1}=W_{2}=W_{s} \bar{W}_{p}+W_{p} \bar{W}_{s}
$$

já no espaço de soma e subtração. O que faz com que a atenuação na qual o sistema se torna separável seja

$$
T^{c}=\frac{W_{s} \bar{W}_{p}+W_{p} \bar{W}_{s}}{W_{s} \bar{W}_{p}+W_{p} \bar{W}_{s}-W_{p} \bar{W}_{p}} .
$$

Podemos ver todas estas regiões no gráfico 5.7 .

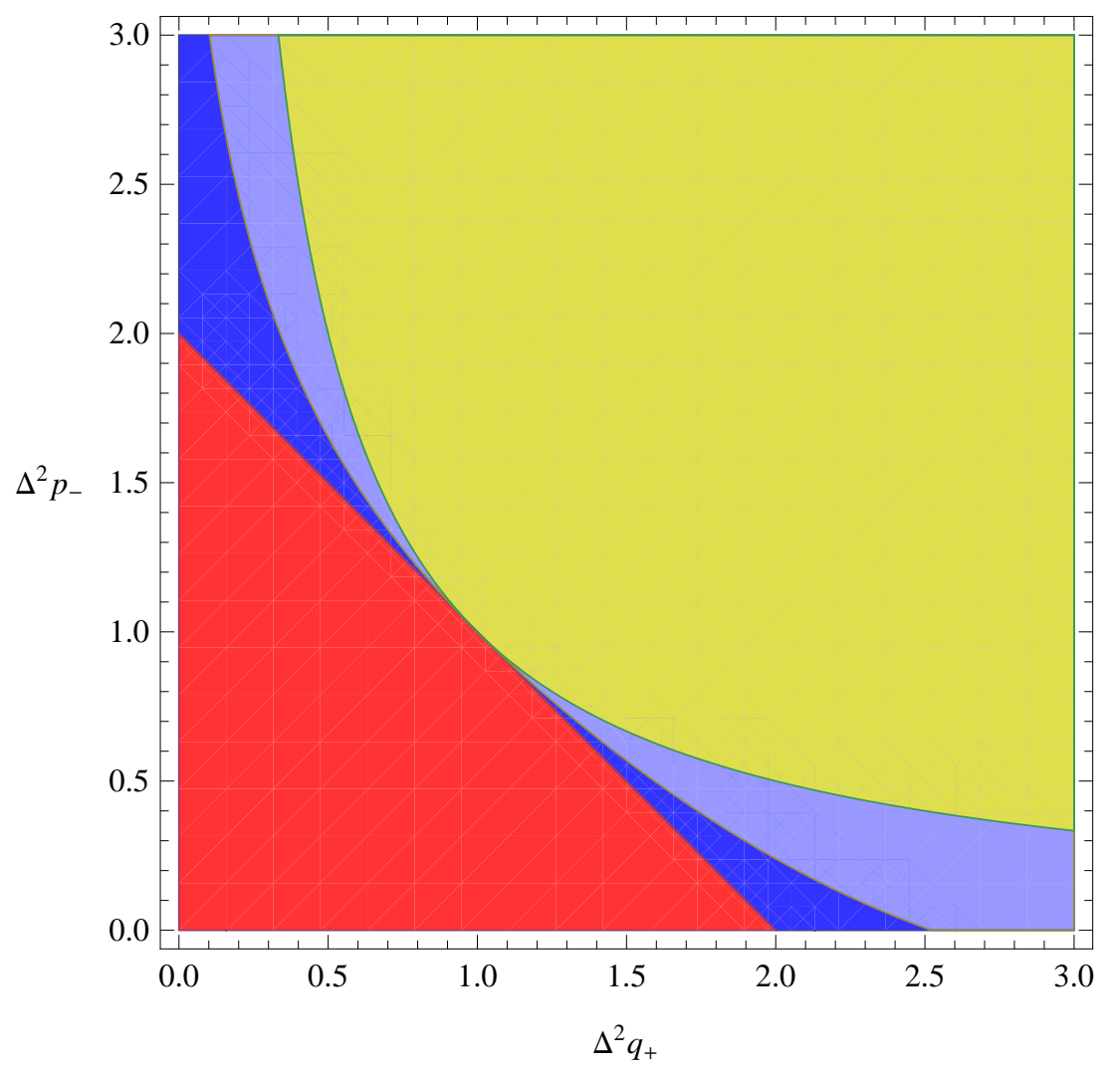

Figura 5.7: As regiões amarela e vermelha representam os estados separáveis e de emaranhamento robusto, respectivamente. Na região azul escura estão os estados parcialmente robustos e na azul clara, os que são frágeis para atenuações em qualquer uma das partes.

Note que, como $p_{ \pm}$e $q_{ \pm}$são pares conjugados, se $W_{p}<0$ então $\bar{W}_{p}>0$ necessa- 
riamente. Em outras palavras, o estado é emaranhado quando apenas um deles é negativo e é separável quando ambos forem positivos. A escolha dentre qual $W_{p}$ e $\bar{W}_{p}$ devemos usar depende de como os subsistemas estão correlacionados. No estado $E P R$, a subtração das posições e a soma dos momentos eram bem conhecidas, portanto, as variâncias de $p_{-}$e $q_{+}$devem possuir squeezing 29 e devemos escolher $W_{p}<0$ como critério de emaranhamento. Isto é precisamente isto que ocorre no nosso OPO, pois temos squeezing na subtração das intensidades e, para potências próximas do limiar, squeezing na soma das fases. De fato, não é estritamente necessário possuir squeezing em ambos $p_{-}$e $q_{+}$, mas apenas em um deles, desde que este seja grande o suficiente para compensar o excesso de ruído presente no outro, fazendo com que $W_{p}<0$. Para um estado no qual esperamos ter squeezing na soma das posições e na subtração dos momentos, o emaranhamento vai ocorrer quando $\bar{W}_{p}<0$. No final, a escolha se resume a saber quais são os pares EPR corretos: $p_{-}$e $q_{+}$ou $p_{+}$e $q_{-}$. As mesmas ideias se aplicam quando analisamos a robustez do emaranhamento, por meio de $W_{R E}$. Quando escolhemos os pares EPR corretos, o critério PPT se iguala ao critério proposto por Giovannetti e Mancini [103], baseado no produto de variâncias, e $W_{R E}<0$ se torna equivalente ao critério DGCZ [89], baseado em soma de variâncias. Ambos são conhecidos na literatura e derivados de forma completamente diferente.

\subsubsection{Comentário Sobre Estados Não-Gaussianos}

Quando os estados não são gaussianos, a condição $W_{p p t} \geq 0$ pode ser satisfeita também para estados emaranhados. Quando atenuada, esta vai continuar positiva, pois é a condição de separabilidade para o estado gaussiano com a mesma matriz de covariâncias deste estado não-gaussiano. Como o emaranhamento presente nestes estados é insensível ao critério $P P T$, a robustez dos mesmos não pode ser corretamente analisada utilizando o critério PPT. A conexão entre estados destiláveis e PPT já é conhecida há um bom tempo: todo estado que possui transposta parcial positiva (PPT), se emaranhado, é não-destilável [125]. A prova da existência (ou inexistência) de estados com transposta parcial negativa (NPT) e com emaranhamento não-destilável é um problema aberto [126]. Entretanto, recentemente foi publicado um resultado que alega ter resolvido este problema [127]. Caso este resultado seja verificado, podemos fazer uma associação entre distilabilidade e violação do critério PPT, portanto, estados para os quais $W_{p p t} \geq 0$ não são destiláveis e, consequentemente, não são úteis em diversos pro-

\footnotetext{
${ }^{29} \mathrm{Ou}$ se anular por completo como no caso ideal tratado por EPR.
} 
tocolos de comunicação quântica. Nestas condições, se o estado não-gaussiano inicial satisfaz o critério PPT, mas passa a violá-lo para alguma atenuação, duas coisas podem ter ocorrido: ele se tornou separável ou evoluiu para um estado cujo emaranhamento não é detectado pelo critério PPT. Qualquer uma das possibilidades não é desejada no contexto de comunicação quântica, pois se o estado continua emaranhado, o emaranhamento se tornou não-destilável. Por outro lado, quando $W_{R E}<0$ o emaranhamento é robusto seja o estado gaussiano ou não-gaussiano, pois o critério $P P T$ é suficiente para o emaranhamento em qualquer tipo de estado quântico. Portanto, se o estado analisado for não-gaussiano, $W_{R E}<0$ é condição somente suficiente para a robustez do emaranhamento e, caso as conclusões de [127] sejam válidas, condição necessária e suficiente para que o emaranhamento destilável seja robusto.

No próximo capítulo, vou tratar da descrição quântica que usamos para o OPO. A partir dela, foi possível prever a existência do emaranhamento tripartite no nosso sistema [14], o que levou a observação, em um primeiro momento, de correlações quânticas entre o campo de bombeio e os feixes gêmeos [16]. Também, devido a discrepâncias entre os dados experimentais e as previsões teóricas, foi possível reconhecer o papel que os fônons do cristal possuem na introdução de ruído de fase [17] nos três campos. Este entendimento propiciou a observação de emaranhamento entre os três campos produzidos pelo OPO [26]. 


\section{Descrição Teórica do OPO Triplamente Ressonante}

\subsection{Descrição Quântica dos Processos Físicos Envolvidos}

Como discutido no capítulo2, o OPO é formado por um cristal não-linear no interior de uma cavidade ótica ressonante. Na descrição quântica, o sistema total é formado pelos campos dentro e fora da cavidade. Os campos intracavidade interagem com os campos do lado externo por meio do espelho de acoplamento e interagem entre si mediados pelo cristal. Os operadores $\hat{a}_{0}, \hat{a}_{1}$ e $\hat{a}_{2}$ descrevem três dos modos ressonantes da cavidades - e representam bombeio, sinal e complementar respectivamente. Eles são definidos pelas relações de comutação,

$$
\left[\hat{a}_{i}, \hat{a}_{j}^{\dagger}\right]=\delta_{i j}
$$

Os campos externos são representados por um conjunto muito grande de osciladores harmônicos desacoplados, descritos por $\hat{b}_{i}^{k}$, onde $i=0,1,2$ representa cada modo intracavidade e o índice $k=0,1, \ldots, N, \operatorname{com} N \rightarrow \infty$, representa os modos do reservatório que interagem com cada modo $\hat{a}_{i}$. As relações de comutação para os modos do reservatório são

$$
\left[\hat{b}_{i}^{k},\left(\hat{b}_{j}^{l}\right)^{\dagger}\right]=\delta_{i j} \delta_{k l} .
$$

Um número grande de modos interage com cada modo intracavidade, pois a parte externa pode ser modelada por uma cavidade de tamanho muito grande e irá agir como um reservatório. O hamiltoniano livre total do sistema é formado pelos hamiltonianos livres da cavidade e do reservatório,

$$
\hat{H}_{\text {livre }}=\sum_{i} \hat{H}_{\text {cav }}^{i}+\hat{H}_{\text {res }}^{i} \text { onde } \hat{H}_{\text {cav }}^{i}=\hbar \omega_{i}^{c}\left[\hat{a}_{i}^{\dagger} \hat{a}_{i}+\frac{1}{2}\right] \text { e } \hat{H}_{\text {res }}^{i}=\sum_{k} \hbar \omega_{i k}\left[\left(\hat{b}_{i}^{k}\right)^{\dagger} \hat{b}_{i}^{k}+\frac{1}{2}\right] \text {. }
$$


Numa situação típica de laboratório, a cavidade é bombeada por feixes laser coerentes. Como discutido no capítulo 3, o estado do campo externo pode ser separado em portadora e bandas laterais no estado de vácuo, $|\psi\rangle_{i}=\left|\alpha_{i p} \mathrm{e}^{i \omega_{i k_{p}}{ }}\right\rangle \otimes \prod_{k \neq k_{p}}|0\rangle_{k}$.

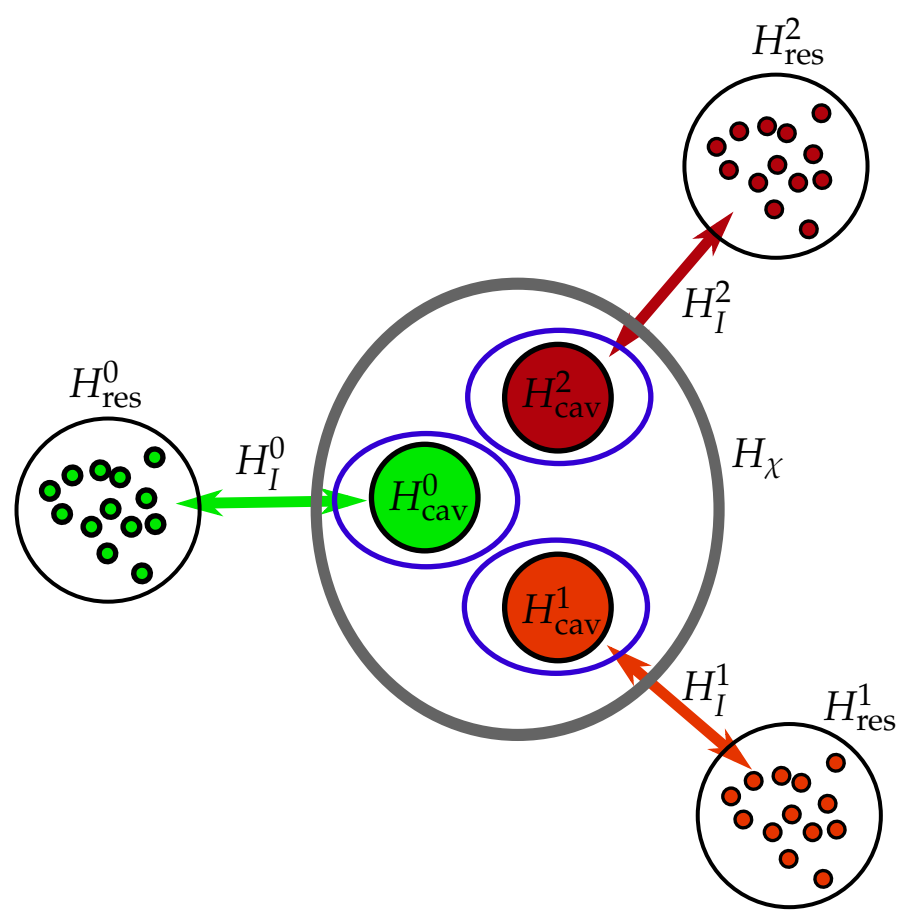

Figura 6.1: Figura esquemática mostrando as interações que ocorrem no OPO. Os círculos maiores verde, vermelho e laranja representam os modos intracavidade e seus respectivos hamiltonianos de evolução livre. O círculo cinza que os cerca representa o hamiltoniano de interação $\hat{H}_{\chi}$ introduzido pelo cristal. Os círculos menores representam os reservatórios que interagem com os modos intracavidade por meio do hamiltoniano $\hat{H}_{I}$.

O hamiltoniano de interação devido à $P D C$ é função somente dos três campos intracavidade. Ele teria uma forma semelhante a uma operação de squeezing de dois modos se o campo de bombeio fosse considerado um campo clássico,

$$
\hat{H}_{\chi}=2 i \hbar \frac{\chi}{\tau}\left[\hat{a}_{0} \hat{a}_{1}^{\dagger} \hat{a}_{2}^{\dagger}-\hat{a}_{0}^{\dagger} \hat{a}_{1} \hat{a}_{2}\right]
$$

onde $\tau=L / c$ é o tempo de voo da frente de onda dentro da cavidade. Este é um modelo efetivo para o processo de conversão paramétrica descendente, cuja constante de acoplamento é função dos tensores de susceptibilidade linear e não-linear de segunda ordem do cristal [64, 65]. O primeiro termo da expressão (6.4) representa de fato a $P D C$, pois um fóton do bombeio é absorvido e os dois fótons gêmeos são emitidos 1 . $\mathrm{O}$ segundo termo é o seu processo inverso, a geração de soma de frequências, no qual os

\footnotetext{
${ }^{1}$ Aqui o casamento de fases é considerado como perfeito, pois a sua largura é muito maior que as larguras típicas das nossas cavidades.
} 
dois fótons gêmeos são recombinados e um fóton é emitido no campo de bombeio. A constante de acoplamento $2 i \hbar \frac{\chi}{\tau}$ é escrita desta forma somente por conveniência ${ }^{2}$.

Cada campo intracavidade interage independentemente com o seu reservatório. Este acoplamento, mediado pelo espelho de entrada/saída, entre o campo $\hat{a}_{i}$ e cada $\hat{b}_{i}^{k}$, é descrito por um modelo de Jaynes-Cummings [73, 128],

$$
\hat{H}_{I}=\hat{H}_{I}^{0}+\hat{H}_{I}^{1}+\hat{H}_{I}^{2}, \quad \operatorname{com} \quad \hat{H}_{I}^{i}=\hbar \sum_{k}\left[g_{i}^{k} \hat{a}_{i}\left(\hat{b}_{i}^{k}\right)^{\dagger}+\left(g_{i}^{k}\right)^{*} \hat{a}_{i}^{\dagger} \hat{b}_{i}^{k}\right]
$$

Este hamiltoniano descreve o processo de absorção (emissão) de um fóton fora da cavidade junto com a emissão (absorção) de outro dentro cavidade. A constante de acoplamento $g_{i}^{k}$ está relacionada com a transmissão dos espelhos.

Vamos analisar primeiro o acoplamento descrito pelo hamiltoniano (6.5). Como mostrado em [73, 128], podemos eliminar os graus de liberdade associados a $\hat{b}_{i}^{k}$ no limite de $k$ contínuo. Consideramos que o estado do sistema fechado cavidade+reservatório está em um estado inicial

$$
\hat{\rho}_{\text {total }}\left(t_{0}\right)=\hat{\rho}_{c}\left(t_{0}\right) \otimes \hat{\rho}_{p}\left(t_{0}\right) \otimes \hat{\rho}_{B}
$$

onde $\hat{\rho}_{c}\left(t_{0}\right)$ é o estado inicial do campo intracavidade, $\hat{\rho}_{p}=\prod_{j}\left|\alpha_{j p} \mathrm{e}^{i \omega_{i p} t_{0}}\right\rangle\left\langle\alpha_{j p} \mathrm{e}^{i \omega_{j p} t_{0}}\right| \mathrm{e}$ $\hat{\rho}_{B}=\prod_{\omega \neq \omega_{p}}|0\rangle_{\omega}\left\langle\left. 0\right|_{\omega}\right.$ é o estado do reservatório 3 . Como o mesmo possui muitos graus de liberdade, ele é grande o suficiente para que seu estado não mude de forma apreciável devido à interação com a cavidade. Neste limite, o acolamento devido ao hamiltoniano (6.5) é reduzido a duas contribuições efetivas. A primeira delas ocorre como um termo de decaimento [62, 73, 128],

$$
\Lambda_{i}(\hat{\rho})=\bar{\gamma}_{i}\left[\hat{a}_{i}^{\dagger} \hat{a}_{i} \hat{\rho}+\hat{\rho} \hat{a}_{i}^{\dagger} \hat{a}_{i}-2 \hat{a}_{i} \hat{\rho} \hat{a}_{i}^{\dagger}\right]
$$

que é o superoperador de Lindblad para o hamiltoniano (6.5). A constante $\bar{\gamma}_{i}=\lim _{\Delta \omega \rightarrow \infty}\left|g_{i}^{k}\right|^{2} / \Delta \omega$ é a taxa de decaimento da cavidade e está associada ao coeficiente de transmissão, $T_{i}$, do espelho por $2 \bar{\gamma}_{i}=T_{i} \tau$. Para derivar este resultado considere a evolução para a intensidade média do campo intracavidade, que é proporcional a $\left\langle\hat{a}_{i}^{\dagger} \hat{a}_{i}\right\rangle$. No caso mais simples em que não há conversão paramétrica, a equação para a intensidade média em

\footnotetext{
${ }^{2} \mathrm{~A}$ forma explícita de $\chi$ não é importante, pois ela será associada à potência de limiar da mesma forma que no capítulo 2

${ }^{3}$ Rigorosamente, o resevatório está em um banho térmico, $\hat{\rho}_{B}=\prod_{i} \mathrm{e}^{-\beta \hat{H}_{\mathrm{res}}^{i}} / \operatorname{Tr}\left[\prod_{i} \mathrm{e}^{-\beta \hat{H}_{\mathrm{res}}^{i}}\right]$, com $\beta=$ $1 / k_{B} T$. Entretanto, em nosso caso, o número médio de fótons é tão pequeno que o estado térmico pode ser aproximado pelo estado de vácuo. Para se ter uma ideia, o número médio de fótons em um estado térmico é $\bar{n}=\left(\mathrm{e}^{\beta \hbar \omega}-1\right)^{-1}$. No domínio ótico, $\lambda$ está entre $0.4 \mu \mathrm{m}$ e $1 \mu \mathrm{m}$, o que faz com que $\beta \hbar \omega$ esteja entre 45 e 113 , ou seja, $\bar{n}<\mathrm{e}^{-45} \approx 10^{-19}$.
} 
cada modo se torna

$$
\frac{d\left\langle\hat{I}_{i}(t)\right\rangle}{d t}=\operatorname{Tr}\left[\hat{a}_{i}^{\dagger} \hat{a}_{i} \frac{d \hat{\rho}}{d t}\right]=-2 \bar{\gamma}_{i}\left\langle\hat{I}_{i}(t)\right\rangle
$$

cuja solução é $\left\langle\hat{I}_{i}(t)\right\rangle=\mathrm{e}^{-2 \bar{\gamma}_{i} t}\langle\hat{I}(0)\rangle$. Após uma volta pela cavidade, teremos $\langle\hat{I}(\tau)\rangle=$ $\mathrm{e}^{-2 \bar{\gamma}_{i} \tau}\langle\hat{I}(0)\rangle$. A cada volta pela cavidade a intensidade vai diminuir por um fator de $\mathrm{e}^{-2 \bar{\gamma}_{i} \tau}$. Esta diminuição na intensidade ocorre devido à transmissão pelo espelho de acoplamento e às perdas espúrias no interior da cavidade. Supondo, por enquanto, que somente há perdas pelo espelho de acoplamento, o coeficiente de reflexão se iguala a $e^{-2 \bar{\gamma}_{i} \tau}$. Consequentemente, o coeficiente de transmissão se torna

$$
T_{i}=1-R_{i}=1-\mathrm{e}^{-2 \gamma_{i}} \approx 2 \gamma_{i}, \quad \text { quando } \gamma_{i}<<\gamma_{i}^{2}
$$

onde $\bar{\gamma}_{i}=\gamma_{i} \tau$. Quando a cavidade não é perfeita, as perdas totais a cada volta passam a ser maiores e $\bar{\gamma}_{i}=\gamma_{i}^{\prime} \tau$, com $\gamma_{i}^{\prime}=\gamma_{i}+\mu_{i}$, onde $\mu_{i}$ são as perdas espúrias. Sendo assim, o superoperador de Lindblad pode ser escrito como

$$
\Lambda_{i}(\hat{\rho})=\frac{\gamma_{i}^{\prime}}{\tau}\left[\hat{a}_{i}^{\dagger} \hat{a}_{i} \hat{\rho}+\hat{\rho} \hat{a}_{i}^{\dagger} \hat{a}_{i}-2 \hat{a}_{i} \hat{\rho} \hat{a}_{i}^{\dagger}\right],
$$

A outra contribuição aparece na forma de um hamiltoniano de interação de cada campo intracavidade com a portadora do campo fora da cavidade. Como no nosso experimento somente a portadora do campo de bombeio é não nula,

$$
\hat{H}_{\text {pump }}=i \hbar \frac{\sqrt{2 \gamma_{0}}}{\tau}\left[\alpha_{i n}^{*} \mathrm{e}^{i \omega_{0} t} \hat{a}_{0}-h . c\right],
$$

onde $\alpha_{i n}=\alpha_{0 p}$ e $\omega_{0} \equiv \omega_{0 p}$ é a frequência da portadora do campo de bombeio. Desta forma, todas as contribuições para a dinâmica do sistema dependem apenas dos operadores de campo intracavidade. Por conveniência, vamos mudar para as chamadas variáveis lentas, nas quais os fatores que oscilam com a frequência da luz são retirados, e nos preocupamos apenas com as oscilações em torno da mesma, $\hat{a}_{i}(t) \rightarrow \mathrm{e}^{-i \omega_{i} t} \hat{a}_{i}(t)$. Estas variáveis podem ser obtidas pela transformação

$$
\hat{a}_{i}(t) \rightarrow \mathrm{e}^{-i \omega_{i} t \hat{a}_{i}^{\dagger} \hat{a}_{i}} \hat{a}_{i} \mathrm{e}^{i \omega_{i} t \hat{a}_{i}^{\dagger} \hat{a}_{i}}
$$

e é equivalente a passar para o formalismo de interação. As frequências dos feixes gêmeos $\omega_{1}$ e $\omega_{2}$ satisfazem $\omega_{1}+\omega_{2}=\omega_{0}$, portanto, o hamiltoniano (6.4) mantém a mesma forma nas variáveis lentas. O hamiltoniano de campo livre (6.3) vai ganhar mais um termo,

$$
\hat{H}_{0}^{\mathrm{cav}} \rightarrow \hat{H}_{0}=\sum_{i} \hbar \frac{\Delta_{i}}{\tau} \gamma_{i}^{\prime}\left[\hat{a}_{i}^{\dagger} \hat{a}_{i}+\frac{1}{2}\right]
$$


onde $\Delta_{i}=2\left(\omega_{i}^{c}-\omega_{i}\right) / \delta \omega_{i}$ é a duas vezes a dessintonia entre a frequência da luz e a ressonância da cavidade normalizada pela largura de banda $\delta \omega_{i}=2 \gamma_{i}^{\prime} / \tau$ - ou, como mostrado no capítulo 2, a fase após uma volta completa normalizada pelas perdas. Quando juntamos todos estes termos, a equação de Von Neumann se torna

$$
\frac{1}{\tau} \frac{d \hat{\rho}}{d t}=-\frac{i}{\hbar}\left[\hat{H}_{0}+\hat{H}_{\chi}+\hat{H}_{\text {pump }}, \hat{\rho}\right]-\sum_{i} \Lambda_{i}(\hat{\rho})
$$

onde $t$ é medido em unidades do tempo de uma volta completa pela cavidade, $\tau$.

\subsection{Análise Espectral do OPO}

A solução da equação (6.14) fornece a descrição teórica para o estado do campos dentro da cavidade do OPO. Como trabalhamos com variáveis contínuas, vamos buscar soluções de (6.14 no espaço de fase e para isto usaremos a função de Wigner, discutida do capítulo 5. Como não temos acesso ao campo intracavidade, mas somente ao campo no exterior, usaremos as relações de entrada e saída de cavidades [87] para obter os campos fora da cavidade a partir da solução para os campos internos.

\subsubsection{Função de Wigner e Equações de Langevin}

A equação de Von Neumann pode ser resolvida usando a representação da função de Wigner para o operador densidade como mostrada em (5.9),

$$
W\left(\alpha, \alpha^{*}\right)=\frac{1}{\left(\pi^{2} \hbar\right)^{n}} \int_{-\infty}^{\infty} d^{2 n} \beta \mathrm{e}^{-i\left(\beta^{*} \alpha+\beta \alpha^{*}\right)} \chi\left(\beta, \beta^{*}\right), \quad \chi\left(\beta, \beta^{*}\right)=\operatorname{Tr}\left[\mathrm{e}^{i\left(\beta^{*} \hat{a}\right.} \mathrm{e}^{\left.i \beta \hat{a}^{\dagger}\right)} \mathrm{e}^{-\frac{|\beta|^{2}}{2}} \hat{\rho}\right] .
$$

Os operadores de criação multiplicados pelo operador densidade correspondem a operadores diferenciais atuando na função de Wigner. Por exemplo, o operador $\hat{a}_{i} \hat{\rho}$ corresponde a

$$
O\left(\alpha, \alpha^{*}\right) W\left(\alpha, \alpha^{*}\right)=\frac{1}{\left(\pi^{2} \hbar\right)^{n}} \int_{-\infty}^{\infty} d^{2 n} \beta \mathrm{e}^{-i\left(\beta^{*} \alpha+\beta \alpha^{*}\right)} \bar{\chi}\left(\beta, \beta^{*}\right),
$$

com função característica $\bar{\chi}\left(\beta, \beta^{*}\right)=\operatorname{tr}\left[\mathrm{e}^{i \beta^{*} \hat{a}} \mathrm{e}^{i \beta \hat{a}^{\dagger}} \mathrm{e}^{-\frac{|\beta|^{2}}{2}} \hat{a}_{i} \rho\right]$ e que pode ser escrita como

$$
\begin{gathered}
\bar{\chi}\left(\beta, \beta^{*}\right)=\operatorname{Tr}\left[\mathrm{e}^{i\left(\beta^{*} \hat{a}\right.} \mathrm{e}^{\left.i \beta \hat{a}^{+}\right)} \mathrm{e}^{-\frac{|\beta|^{2}}{2}} \hat{a}_{i} \rho\right]=-i\left(\frac{\partial}{\partial \beta_{i}^{*}}+\frac{\beta_{i}}{2}\right) \chi\left(\beta, \beta^{*}\right), \quad \text { portanto, } \\
O\left(\alpha, \alpha^{*}\right) W\left(\alpha, \alpha^{*}\right)=\left(\frac{1}{2} \frac{\partial}{\partial \alpha_{i}^{*}}+\alpha_{i}\right) W\left(\alpha, \alpha^{*}\right)+\frac{1}{\left(\pi^{2} \hbar\right)^{n}} \int_{-\infty}^{\infty} d^{2 n} \beta \frac{\partial}{\partial \beta_{i}^{*}}\left[\mathrm{e}^{-i\left(\beta^{*} \alpha+\beta \alpha^{*}\right)} \chi\left(\beta, \beta^{*}\right)\right] .
\end{gathered}
$$


Como o último termo desta equação se anula devido ao teorema de Stokes, podemos fazer a seguinte associação

$$
\hat{a}_{i} \hat{\rho} \rightarrow O\left(\alpha, \alpha^{*}\right) W\left(\alpha, \alpha^{*}\right)=\left(\frac{1}{2} \frac{\partial}{\partial \alpha_{i}^{*}}+\alpha_{i}\right) W\left(\alpha, \alpha^{*}\right)
$$

Como em (6.18), a multiplicação por operadores de criação e aniquilação equivale a operadores diferenciais na representação da função de Wigner [87, 73],

$$
\hat{\rho} \hat{a}_{i} \rightarrow\left(\alpha_{i}-\frac{1}{2} \frac{\partial}{\partial \alpha_{i}^{*}}\right) W\left(\alpha, \alpha^{*}\right), \quad \hat{a}_{i}^{\dagger} \hat{\rho} \rightarrow\left(\alpha_{i}^{*}-\frac{1}{2} \frac{\partial}{\partial \alpha_{i}}\right) W\left(\alpha, \alpha^{*}\right) \quad \text { e } \quad \hat{\rho} \hat{a}_{i}^{\dagger} \rightarrow\left(\alpha_{i}^{*}+\frac{1}{2} \frac{\partial}{\partial \alpha_{i}}\right) W\left(\alpha, \alpha^{*}\right) .
$$

Quando substituímos estas correspondências para os operadores que compõem a equação de Von Neumann, obtemos uma equação diferencial para a função de Wigner equivalente a equação (6.14),

$$
\frac{\partial W}{\partial t}=-\sum_{i} \frac{\partial}{\partial X_{i}}\left[A_{i} W\right]+\frac{1}{2} \sum_{i, j} \frac{\partial^{2}}{\partial X_{i} \partial X_{j}}\left[\sum_{k} B_{i k} B_{j k} W\right]-\chi\left[\frac{\partial^{3} W}{\partial \alpha_{0}^{*} \partial \alpha_{1}^{*} \partial \alpha_{2}^{*}}+\text { c.c. }\right],
$$

com o tempo medido em unidades de $\tau$. As quantidades em 6.19 são $X=\left(\alpha_{0}, \alpha_{0}^{*}, \alpha_{1}, \alpha_{1}^{*}, \alpha_{2}, \alpha_{2}^{*}\right)$, a matriz de difusão $B=\mathrm{T}+\mathrm{T}^{\prime} \operatorname{com} \mathrm{T}=\operatorname{diag}\left(\sqrt{2 \gamma_{0}}, \sqrt{2 \gamma_{0}}, \sqrt{2 \gamma_{1}}, \sqrt{2 \gamma_{1}}, \sqrt{2 \gamma_{2}}, \sqrt{2 \gamma_{2}}\right)$ e $\mathrm{T}^{\prime}=\operatorname{diag}\left(\sqrt{2 \mu_{0}}, \sqrt{2 \mu_{0}}, \sqrt{2 \mu_{1}}, \sqrt{2 \mu_{1}}, \sqrt{2 \mu_{2}}, \sqrt{2 \mu_{2}}\right)$ e o vetor de arrasto

$$
A(X)=\left(\begin{array}{c}
-\gamma_{0}^{\prime}\left(1-i \Delta_{0}\right) \alpha_{0}-2 \chi \alpha_{1} \alpha_{2}+\sqrt{2 \gamma_{0}} \alpha_{i n} \\
-\gamma_{0}^{\prime}\left(1+i \Delta_{0}\right) \alpha_{0}^{*}-2 \chi \alpha_{1}^{*} \alpha_{2}^{*}+\gamma_{0} \alpha_{i n} \\
-\gamma_{1}^{\prime}\left(1-i \Delta_{1}\right) \alpha_{1}+2 \chi \alpha_{0} \alpha_{2}^{*} \\
-\gamma_{1}^{\prime}\left(1+i \Delta_{1}\right) \alpha_{1}^{*}+2 \chi \alpha_{0}^{*} \alpha_{2} \\
-\gamma_{2}^{\prime}\left(1-i \Delta_{2}\right) \alpha_{2}+2 \chi \alpha_{0} \alpha_{1}^{*} \\
-\gamma_{2}^{\prime}\left(1+i \Delta_{2}\right) \alpha_{2}^{*}+2 \chi \alpha_{0}^{*} \alpha_{1}
\end{array}\right) .
$$

Se desprezamos a derivada terceira, chegamos em uma equação de Fokker-Planck, que podemos transformar em um conjunto de equações de Langevin.

$$
\frac{d X_{i}}{d t}=A_{i}+\sum_{j} T_{i j} \zeta_{j}(t)+T_{i j}^{\prime} \zeta_{j}^{\prime}(t)
$$

onde $\zeta(t)$ e $\zeta^{\prime}(t)$ representam, respectivamente, os estados de vácuo que entram pelo espelho de acoplamento e por todas as outras entradas da cavidade. Eles são modelados como ruídos brancos descorrelacionados entre si: $\left\langle\zeta_{i}(t)\right\rangle=\left\langle\zeta_{i}^{\prime}(t)\right\rangle=0,\left\langle\zeta_{i}(t) \zeta_{j}\left(t^{\prime}\right)\right\rangle=$ $\left\langle\zeta_{i}^{\prime}(t) \zeta_{j}^{\prime}\left(t^{\prime}\right)\right\rangle=2 \pi \delta_{i j} \delta\left(t-t^{\prime}\right)$ e $\left\langle\zeta_{i}(t) \zeta_{j}^{\prime}\left(t^{\prime}\right)\right\rangle=0$ [128, 70, 87].

Vamos separar as amplitudes de campo em valor médio mais flutuações, $\alpha_{i}=$ 
$\bar{\alpha}_{i}+\delta \alpha_{i}$ e desprezar termos de ordem superior. Neste caso, as seguintes equações são equivalentes à equação de von Neumann,

$$
\begin{gathered}
\frac{d \bar{X}_{i}}{d t}=A_{i}(\bar{X}) \quad \text { e } \frac{d\left(\delta X_{i}\right)}{d t}=\sum_{j} M_{i j} \delta X_{j}+\mathrm{T}_{i} \delta \zeta_{i}+\mathrm{T}_{i}^{\prime} \delta \zeta_{i}^{\prime}, \quad \text { com } \\
M=\left(\begin{array}{cccccc}
-\gamma_{0}^{\prime}\left(1-i \Delta_{0}\right) & 0 & 0 & -2 \chi \bar{\alpha}_{2} & 0 & -2 \chi \bar{\alpha}_{1} \\
0 & -\gamma_{0}^{\prime}\left(1+i \Delta_{0}\right) & -2 \chi \bar{\alpha}_{2}^{*} & 0 & -2 \chi \bar{\alpha}_{1}^{*} & 0 \\
2 \chi \bar{\alpha}_{2}^{*} & 0 & -\gamma_{1}^{\prime}\left(1-i \Delta_{1}\right) & 0 & 0 & 2 \chi \bar{\alpha}_{0} \\
0 & 2 \chi \bar{\alpha}_{2} & 0 & -\gamma_{1}^{\prime}\left(1+i \Delta_{1}\right) & 2 \chi \bar{\alpha}_{0}^{*} & 0 \\
2 \chi \bar{\alpha}_{1}^{*} & 0 & 0 & 2 \chi \bar{\alpha}_{0} & -\gamma_{2}^{\prime}\left(1-i \Delta_{2}\right) & 0 \\
0 & 2 \chi \bar{\alpha}_{1} & 2 \chi \bar{\alpha}_{0}^{*} & 0 & 0 & -\gamma_{2}^{\prime}\left(1-i \Delta_{2}\right)
\end{array}\right)
\end{gathered}
$$

As equações (6.22) descrevem a dinâmica dos campos intracavidade na aproximação linearizada As segunda equação de (6.22) descreve a dinâmica das flutuações de quadratura para o OPO abaixo e acima do limiar. O que irá diferenciar os dois regimes de operação são os valores médios. Abaixo do limiar, por exemplo, $\alpha_{1}=\alpha_{2}=0$, o que faz com que a equação (6.22) se desacople em bombeio + gêmeos.

\subsubsection{Solução Espectral para as Flutuações}

Os valores médios são obtidos pela solução da primeira equação de (6.22). Ela é um conjunto de equações não-lineares para $\bar{\alpha}_{i}$ que explicitamente é igual a

$$
\begin{gathered}
\frac{d \bar{\alpha}_{0}}{d t}=-\gamma_{0}^{\prime}\left(1-i \Delta_{0}\right) \bar{\alpha}_{0}-2 \chi \bar{\alpha}_{1} \bar{\alpha}_{2}+\sqrt{2 \gamma_{0}} \alpha_{i n} \\
\frac{d \bar{\alpha}_{1}}{d t}=-\gamma_{1}^{\prime}\left(1-i \Delta_{1}\right) \bar{\alpha}_{1}+2 \chi \bar{\alpha}_{0} \bar{\alpha}_{2}^{*} \quad \text { e } \frac{d \bar{\alpha}_{1}}{d t}=-\gamma_{2}^{\prime}\left(1-i \Delta_{2}\right) \bar{\alpha}_{2}+2 \chi \bar{\alpha}_{0} \bar{\alpha}_{1}^{*} .
\end{gathered}
$$

Estamos interessados na dinâmica temporal das flutuações. Consequentemente, a solução para os valores médios que nos interessa é a obtida para tempos longos 5 . Neste contexto, se há uma solução estável, $\bar{\alpha}_{i}$, tal que $\lim _{t \rightarrow \infty} \bar{\alpha}_{i}(t)=\bar{\alpha}_{i}$, as derivadas temporais se anulam para tempos longos e o conjunto de equações (6.23) e (6.24) se torna $A_{i}(\bar{X})=0$ e são iguais às previstas para o OPO clássico mostradas em 2.37) e (2.38). Portanto, os valores médios para tempos longos são derivados de (2.39), (2.44)

\footnotetext{
${ }^{4}$ Em que os valores médios são bem maiores que as flutuações, $\bar{\alpha}_{i}>>\delta \alpha_{i}$.

${ }^{5}$ Essa solução é por vezes também chamada de solução estacionária. Porém como a palavra estacionária é usada com um sentido muito específico neste trabalho, não usarei esta mesma denominação.
} 
e (2.45)

$$
\begin{gathered}
\bar{\alpha}_{i}=\left|\bar{\alpha}_{i}\right| \mathrm{e}^{i \phi_{i}}, \quad \text { onde } \mathrm{e}^{i\left(\phi_{0}-\phi_{1}-\phi_{2}\right)}=\frac{(1-i \Delta)}{\sqrt{1+\Delta^{2}}}, \quad\left|\bar{\alpha}_{0}\right|^{2}=\frac{2 \gamma_{0} P_{\lim }}{\left(\gamma_{0}^{\prime}\right)^{2}}\left(1+\Delta^{2}\right) \\
\text { e }\left|\bar{\alpha}_{i}\right|^{2}=\frac{2 \gamma_{0} P_{\lim }}{\gamma_{i}^{\prime} \gamma_{0}^{\prime}}\left[\Delta \Delta_{0}-1+\sqrt{\sigma-\left(\Delta+\Delta_{0}\right)^{2}}\right] \text { para } i=1 \mathrm{e} 2,
\end{gathered}
$$

$\operatorname{com} P_{\lim }=\frac{\gamma_{1}^{\prime} \gamma_{2}^{\prime}\left(\gamma_{0}^{\prime}\right)^{2}}{8 \gamma_{0} \chi^{2}}$ e $\sigma=\left|\alpha_{i n}\right|^{2} / P_{\text {lim. }}$. Usamos essa solução para construir a matriz $M$ que fornece a dinâmica das flutuações. Como estamos interessados nas variáveis do espaço de fase, vamos escrever $\delta \alpha_{i}$ e $\delta \alpha_{i}^{*}$ em função de variáveis canônicas. As equações 6.22 podem ser escritas como

$$
\begin{gathered}
\frac{d}{d t} \delta \alpha_{0}=-\gamma_{0}^{\prime}\left(1-i \Delta_{0}\right) \delta \alpha_{0}-2 \chi\left|\bar{\alpha}_{2}\right| \mathrm{e}^{i \phi_{2}} \delta \alpha_{1}-2 \chi\left|\bar{\alpha}_{1}\right| \mathrm{e}^{i \phi_{1}} \delta \alpha_{2}+\sqrt{2 \gamma_{0}} \zeta_{1}+\sqrt{2 \mu_{0}} \zeta_{1}^{\prime} \\
\frac{d}{d t} \delta \alpha_{1}=-\gamma^{\prime}(1-i \Delta) \delta \alpha_{1}+2 \chi\left|\bar{\alpha}_{2}\right| \mathrm{e}^{-i \phi_{2}} \delta \alpha_{0}+2 \chi\left|\bar{\alpha}_{0}\right| \mathrm{e}^{i \phi_{0}} \delta \alpha_{2}^{*}+\sqrt{2 \gamma} \zeta_{3}+\sqrt{2 \mu} \zeta_{3}^{\prime} \\
\frac{d}{d t} \delta \alpha_{2}=-\gamma^{\prime}(1-i \Delta) \delta \alpha_{2}+2 \chi\left|\bar{\alpha}_{1}\right| \mathrm{e}^{-i \phi_{1}} \delta \alpha_{0}+2 \chi\left|\bar{\alpha}_{0}\right| \mathrm{e}^{i \phi_{0}} \delta \alpha_{1}^{*}+\sqrt{2 \gamma} \zeta_{5}+\sqrt{2 \mu} \zeta_{5}^{\prime}
\end{gathered}
$$

e os seus respectivos conjugados complexos. Fazendo as transformações $\delta \alpha_{i} \rightarrow \delta \alpha_{i} \mathrm{e}^{-i \phi_{i}}$, $\zeta_{1} \rightarrow \zeta_{1} \mathrm{e}^{-i \phi_{0}}, \zeta_{1}^{\prime} \rightarrow \zeta_{1}^{\prime} \mathrm{e}^{-i \phi_{0}}$, assim como relações similares para as outras componentes de $\zeta$ e $\zeta^{\prime}$, podemos usar a relação entre as fases dos campos médios mostrada em 6.25) para eliminá-las das equações 6.27 6.29,

$$
\begin{aligned}
& \frac{d}{d t} \delta \alpha_{0}=-\gamma_{0}^{\prime}\left(1-i \Delta_{0}\right) \delta \alpha_{0}-2 \chi|\bar{\alpha}| \frac{(1+i \Delta)}{\sqrt{1+\Delta^{2}}}\left(\delta \alpha_{1}+\delta \alpha_{2}\right)+\sqrt{2 \gamma_{0}} \zeta_{1}+\sqrt{2 \mu} \zeta_{1}^{\prime}, \\
& \frac{d}{d t} \delta \alpha_{1}=-\gamma^{\prime}(1-i \Delta) \delta \alpha_{1}+2 \chi \frac{(1-i \Delta)}{\sqrt{1+\Delta^{2}}}\left(|\bar{\alpha}| \delta \alpha_{0}+\left|\bar{\alpha}_{0}\right| \delta \alpha_{2}^{*}\right)+\sqrt{2 \gamma} \zeta_{3}+\sqrt{2 \mu} \zeta_{3^{\prime}}^{\prime} \\
& \frac{d}{d t} \delta \alpha_{2}=-\gamma^{\prime}(1-i \Delta) \delta \alpha_{2}+2 \chi \frac{(1-i \Delta)}{\sqrt{1+\Delta^{2}}}\left(|\bar{\alpha}| \delta \alpha_{0}+\left|\bar{\alpha}_{0}\right| \delta \alpha_{1}^{*}\right)+\sqrt{2 \gamma} \zeta_{5}+\sqrt{2 \mu} \zeta_{5}^{\prime} .
\end{aligned}
$$

Esta transformação tem uma interpretação física muito importante: medimos as flutuações em relação à fase média do campo. Se as fases médias dos campos fossem fixas no tempo, esta mudança de variável seria somente uma conveniência matemática. Porém, como ocorre em qualquer laser, as fases médias dos campos sofrem um processo de difusão equivalente a um passeio aleatório fazendo com que, em cada medida, as fases $\phi_{i}$ sejam diferentes. Entretanto, as flutuações em $\alpha_{i}$ são medidas tendo esta fase como referência.

As quadraturas que correspondem às flutuações de fase e intensidade são

$$
\delta q_{i}=\delta \alpha_{i}^{*}+\delta \alpha_{i} \quad \text { e } \quad \delta p_{i}=i\left(\delta \alpha_{i}^{*}-\delta \alpha_{i}\right)
$$


e representam operadores hermitianos com relações de comutação de posição e momento ${ }^{6}$ As equações do OPO em função das combinações reais $\delta q_{i}$ e $\delta p_{i}$ se tornam

$$
\begin{gathered}
\frac{d}{d t} \delta q_{0}=-\gamma_{0}^{\prime} \delta q_{0}-\gamma_{0}^{\prime} \Delta_{0} \delta p_{0}-\eta\left[\delta q_{1}+\delta q_{2}-\Delta\left(\delta p_{1}+\delta p_{2}\right)\right]+\sqrt{2 \gamma_{0}} v_{0}^{q}+\sqrt{2 \mu_{0}} v_{0}^{q^{\prime}}, \\
\frac{d}{d t} \delta p_{0}=-\gamma_{0}^{\prime} \delta p_{0}+\gamma_{0}^{\prime} \Delta_{0} \delta q_{0}-\eta\left[\delta p_{1}+\delta p_{2}+\Delta\left(\delta q_{1}+\delta q_{2}\right)\right]+\sqrt{2 \gamma v_{0}} v_{0}^{p}+\sqrt{2 \mu_{0}} v_{0}^{p^{\prime}}, \\
\frac{d}{d t} \delta q_{1}=-\gamma^{\prime}\left(\delta q_{1}-\delta q_{2}\right)-\gamma^{\prime} \Delta\left(\delta p_{1}+\delta p_{2}\right)+\eta\left(\delta q_{0}+\Delta \delta p_{0}\right)+\sqrt{2 \gamma} v_{1}^{q}+\sqrt{2 \mu} v_{1}^{q^{\prime}}, \\
\frac{d}{d t} \delta p_{1}=-\gamma^{\prime}\left(\delta p_{1}+\delta p_{2}\right)+\gamma^{\prime} \Delta\left(\delta q_{1}-\delta q_{2}\right)+\eta\left(\delta p_{0}-\Delta \delta q_{0}\right)+\sqrt{2 \gamma} v_{1}^{p}+\sqrt{2 \mu} v_{1}^{p,}, \\
\frac{d}{d t} \delta q_{2}=-\gamma^{\prime}\left(\delta q_{2}-\delta q_{1}\right)-\gamma^{\prime} \Delta\left(\delta p_{2}+\delta p_{1}\right)+\eta\left(\delta q_{0}+\Delta \delta p_{0}\right)+\sqrt{2 \gamma} v_{2}^{q}+\sqrt{2 \mu} v_{2}^{q \prime}, \\
\frac{d}{d t} \delta p_{2}=-\gamma^{\prime}\left(\delta p_{2}+\delta p_{1}\right)+\gamma^{\prime} \Delta\left(\delta q_{2}-\delta q_{1}\right)+\eta\left(\delta p_{0}-\Delta \delta q_{0}\right)+\sqrt{2 \gamma} v_{2}^{p}+\sqrt{2 \mu} v_{2}^{p,},
\end{gathered}
$$

onde a constante $\chi$ foi eliminada por meio de $2 \chi=\gamma_{0}^{\prime} \gamma^{\prime} / \sqrt{2 \gamma_{0} P_{\text {lim }}} \mathrm{e}$

$$
\left.\eta=\frac{\gamma_{0}^{\prime} \gamma^{\prime}|\bar{\alpha}|}{\sqrt{2 \gamma_{0} P_{\lim }\left(1+\Delta^{2}\right)}}=\frac{\sqrt{\gamma_{0}^{\prime} \gamma^{\prime}}}{\sqrt{1+\Delta^{2}}}\left[\Delta \Delta_{0}-1+\sqrt{\sigma-\left(\Delta+\Delta_{0}\right.}\right)^{2}\right] .
$$

Aqui também já considero o caso particular em que as perdas para os feixes gêmeos são iguais, $\gamma_{1}=\gamma_{2}$ e $\mu_{1}=\mu_{2}$. As quantidades $v_{i}^{q}$ e $v_{i}^{p}\left(v_{i}^{q \prime}\right.$ e $\left.v_{i}^{p \prime}\right)$ são combinações de $\zeta_{i}$ $\left(\zeta_{i}^{\prime}\right)$ resultantes de 6.30 e também são números reais. Nestas equações, podemos ver como as variáveis $E P R, \delta q_{1}-\delta q_{2}$ e $\delta p_{1}+\delta p_{2}$, aparecem naturalmente, especialmente nas equações referentes aos feixes gêmeos. Chegamos então em um conjunto de equações de Langevin acopladas, cujos coeficientes não dependem da potência de limiar, somente das perdas intracavidade, das dessintonias e de $\sigma$ - todos parâmetros que temos acesso direto no experimento. Elas podem ser escritas em uma notação matricial como

$$
\begin{gathered}
\frac{d(\delta X)}{d t}=\bar{M} \delta X+\mathrm{T}^{\mathrm{T}} \delta v+\mathrm{T}^{\mathrm{T}} \delta v^{\prime}, \text { onde } \\
\bar{M}=\left(\begin{array}{cccccc}
-\gamma_{0}^{\prime} & -\gamma_{0}^{\prime} \Delta_{0} & -\eta & \eta \Delta & -\eta & \eta \Delta \\
\gamma_{0}^{\prime} \Delta_{0} & -\gamma_{0}^{\prime} & -\eta \Delta & -\eta & -\eta \Delta & -\eta \\
\eta & \eta \Delta & -\gamma^{\prime} & -\gamma^{\prime} \Delta & \gamma^{\prime} & -\gamma^{\prime} \Delta \\
-\eta \Delta & \eta & \gamma^{\prime} \Delta & -\gamma^{\prime} & -\gamma^{\prime} \Delta & -\gamma^{\prime} \\
\eta & \eta \Delta & \gamma^{\prime} & -\gamma^{\prime} \Delta & -\gamma^{\prime} & -\gamma^{\prime} \Delta \\
-\eta \Delta & \eta & -\gamma^{\prime} \Delta & -\gamma^{\prime} & \gamma^{\prime} \Delta & -\gamma^{\prime}
\end{array}\right),
\end{gathered}
$$

${ }^{6}$ As quantidades $\delta q_{i}$ e $\delta \mathrm{p}_{i}$ mostradas na equação anterior não são operadores. Elas são os parâmetros da função de Wigner, como mostrado em (5.8), e suas médias estatísticas, tendo a função de Wigner como densidade de quasi-probabilidade são equivalentes às média quânticas dos operadores no espaço de fase. 
e $\delta X=\left(\delta q_{0}, \delta p_{0}, \delta q_{1}, \delta p_{1}, \delta q_{2}, \delta p_{2}\right)^{T}$. Como o conjunto de equações é linear em $\delta X$, resolvemos este no domínio da frequência,

$$
\delta X(\Omega)=-\frac{1}{2 i \gamma^{\prime} \Omega+\bar{M}}\left[\mathrm{~T} \delta v(\Omega)+\mathrm{T}^{\prime} \delta v(\Omega)^{\prime}\right], \quad \text { onde } \quad \delta X(\Omega)=\int_{-\infty}^{+\infty} d t \delta X(t) \mathrm{e}^{-2 i \gamma^{\prime} \Omega t}
$$

e $\Omega$ é medida em unidades da largura de banda da cavidade do OPO para os feixes gêmeos 7 . As quantidades $v(\Omega)$ e $v(\Omega)^{\prime}$ também são transformadas de Fourier de $v(t)$ e $v(t)^{\prime}$. Como as mesmas representam as bandas laterais do campo de entrada na cavidade, elas são processos estacionários, pois $\left\langle v_{i}(\Omega) v_{j}\left(\Omega^{\prime}\right)\right\rangle=\left\langle v_{i}(\Omega)^{\prime} v_{j}\left(\Omega^{\prime}\right)^{\prime}\right\rangle=$ $2 \pi \delta_{i j} \delta\left(\Omega+\Omega^{\prime}\right)$ e $\left\langle v_{i}(\Omega)\right\rangle=\left\langle v_{j}(\Omega)^{\prime}\right\rangle=0$. Esta é a solução espectral para as flutuações dos campos intracavidade. Entretanto, temos acesso no experimento somente aos campos transmitidos. Podemos fazer a conversão entre os dois campos por meio dos processos de entrada/saída de cavidade [128, 73]. Na descrição clássica, o campo transmitido vai ser multiplicado por um fator igual à raiz do coeficiente de transmissão. Porém, na descrição quântica, outro termo, referente à reflexão do vácuo externo à cavidade, deve ser adicionado,

$$
\delta X_{\mathrm{S}}(\Omega)=\mathrm{T} \delta X(\Omega)-\delta v(\Omega)
$$

na aproximação em que estamos trabalhando, $T_{\text {bombeio }} \approx \sqrt{2 \gamma_{0}}<<1$ e $T_{\text {gêmeos }} \approx \sqrt{2 \gamma}<<$ 1 , consequentemente, $R_{\text {bombeio }} \approx 1$ e $R_{\text {gêmeos }} \approx 1$. Portanto, o campo que medimos de fato em cada fotodetetor é

$$
\delta X_{\mathrm{s}}(\Omega)=-\left[\mathrm{T} \frac{1}{2 \gamma^{\prime} i \Omega+\bar{M}} \mathrm{~T}+1\right] \delta v(\Omega)-\mathrm{T} \frac{1}{2 \gamma^{\prime} i \Omega+\bar{M}} \mathrm{~T}^{\prime} \delta v(\Omega)^{\prime} .
$$

Toda a informação sobre as propriedades quânticas espectrais do sistema estão presentes em $\delta X_{\mathrm{S}}(\Omega)$, pois os valores médios nos fornecem somente a dinâmica clássica. Neste sistema, o espectro de ruído, $S_{\mathrm{S}}(\Omega)=\left\langle\delta X_{\mathrm{S}}(\Omega) \delta X_{\mathrm{S}}(-\Omega)\right\rangle$, se torna

$$
S_{\mathrm{S}}(\Omega)=\left[\mathrm{T} \frac{1}{2 \gamma^{\prime} i \Omega+\bar{M}} \mathrm{~T}+1\right]\left[\mathrm{T} \frac{1}{-2 \gamma^{\prime} i \Omega+\bar{M}} \mathrm{~T}+1\right]+\mathrm{T} \frac{1}{2 \gamma^{\prime} i \Omega+\bar{M}} \mathrm{~T}^{\prime 2} \frac{1}{-i \Omega+\bar{M}} \mathrm{~T}
$$

e descreve, em primeira aproximação, as propriedades quânticas de um sistema geral. Caso o estado seja gaussiano e estacionário, todas estas propriedades do sistema estão presentes em $S_{\mathrm{s}}(\Omega)$, conforme discutido no capítulo 2. No caso particular em que

\footnotetext{
${ }^{7} \mathrm{O}$ fator $2 \gamma^{\prime}$ que aparece na exponencial em (6.39) é introduzido para fazer esta conversão, pois, como $t$ é medido em unidades de $\tau, 2 \gamma^{\prime} \Omega$ é a frequência angular em unidades do FSR. O que faz com que $\Omega$ esteja definida em unidades de largura de banda.
} 

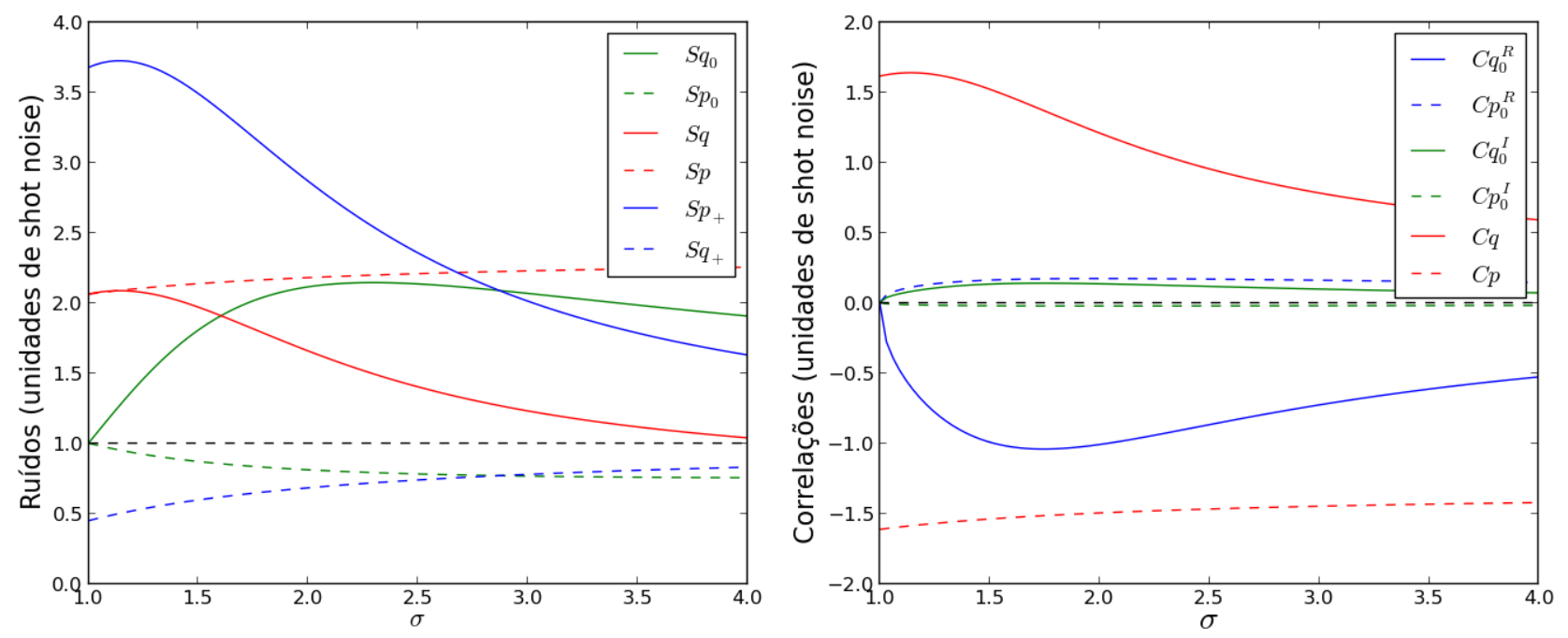

Figura 6.2: Ruídos do OPO - Previsão teórica para os ruídos e correlações dos campos poduzidos pelo OPO.

$\Delta=\Delta_{0}=0$, o espectro de ruído possui a seguinte forma,

$$
S_{\mathrm{S}}(\Omega)=\left(\begin{array}{cccccc}
S q_{0} & 0 & C q_{0}^{R}+i C q_{0}^{I} & 0 & C q_{0}^{R}+i C q_{0}^{I} & 0 \\
0 & S p_{0} & 0 & C p_{0}^{R}+i C p_{0}^{I} & 0 & C p_{0}^{R}+i C p_{0}^{I} \\
C q_{0}^{R}-i C q_{0}^{I} & 0 & S q & 0 & C q & 0 \\
0 & C p_{01}^{R}-i C p_{01}^{I} & 0 & S p & 0 & C p \\
C q_{0}^{R}-i C q_{0}^{I} & 0 & C q & 0 & S q & 0 \\
0 & C p_{0}^{R}-i C p_{02}^{I} & 0 & C p & 0 & S p
\end{array}\right)
$$

e suas entradas são mostradas na figura 6.2

\subsubsection{Emaranhamento e Excesso de Ruído de Fase Introduzido pelos Fônons}

A teoria descrita até aqui concorda quantitativamente muito bem com o experimento para os ruídos e correlações de intensidade. Porém no que diz respeito à fase há excesso de ruído e correlação presentes nos três campos [129]. Este problema foi analisado em detalhes em [17] e tem como causa o espalhamento de fótons da portadora para as bandas laterais devido ao acoplamento com os fônons no cristal. Consequentemente, este efeito deve ser menor quanto mais frio estiver o cristal. Nestas condições, os ruídos e correlações dos campos intracavidade são acrescidos das entradas da matriz 

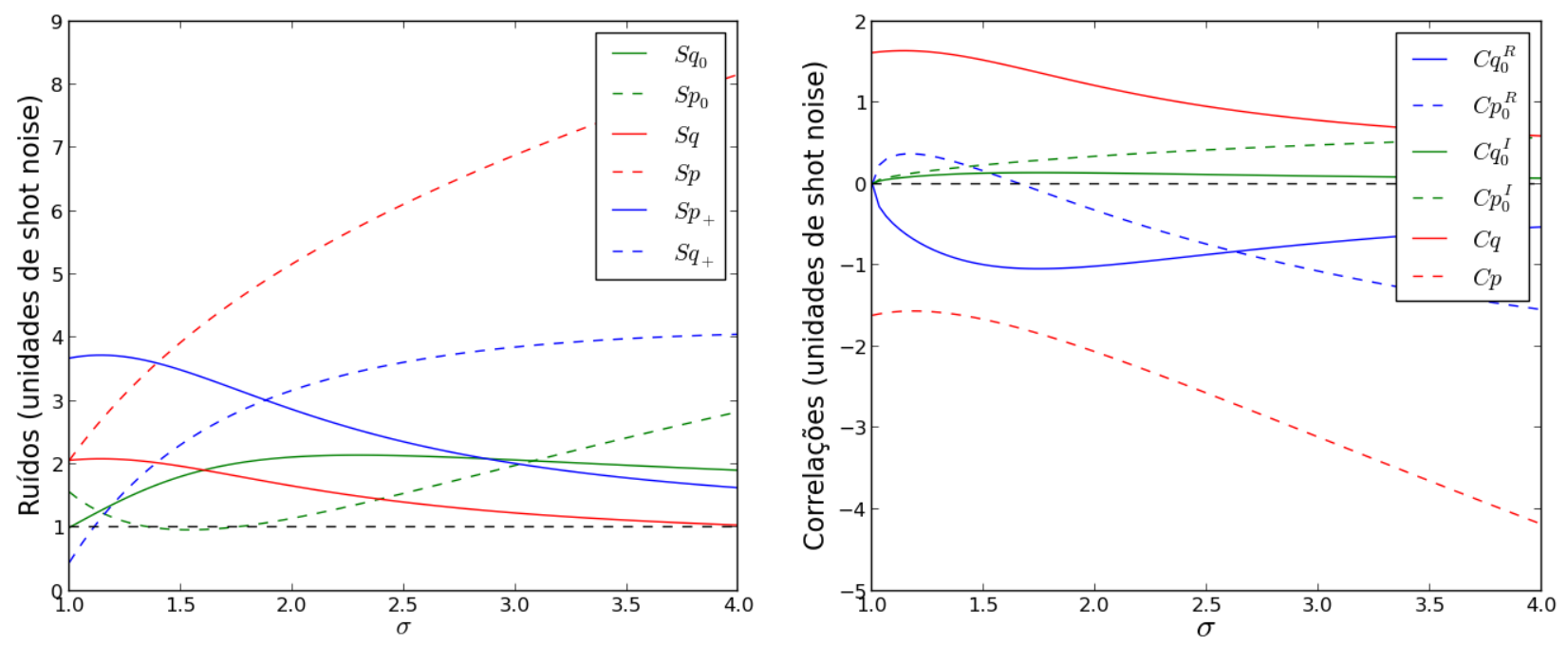

Figura 6.3: Ruídos do OPO - Situação em que adicionamos o ruído de fase introduzido pelos fônons do cristal.

de difusão espectral,

$$
V_{i j}^{Q}(\Omega)=\eta_{i j} \sqrt{P_{i} P_{j}}
$$

Isto faz com que a matriz espectral na saída seja dada por

$$
S_{p h}(\Omega)=S_{s}(\Omega)+\mathrm{T} \frac{1}{2 \gamma^{\prime} i \Omega+\bar{M}} V^{\mathrm{Q}} \frac{1}{-i \Omega+\bar{M}} \mathrm{~T}
$$

Os coeficientes $\eta_{i}$ podem ser medidos de forma independente do OPO e são função da temperatura, como mostrado em [17]. Note que como não há menção $\sigma$ em (6.44), esta contribuição para a matriz de covariâncias espectral depende explicitamente da potência de limiar.

A matriz de covariâncias global composta pelas seis bandas laterais dos três campos está relacionada com o espectro de ruído pela relação (3.122). De posse da matriz de covariâncias, podemos testar o critério PPT como discutido no capítulo5. De acordo com (3.123), a parte real do espectro de ruído se refere à matriz de covariâncias dos operadores no subespaço de soma de bandas laterais (ou subtração a menos de uma rotação) quando a fotocorrente é estacionária

$$
V_{+}=\operatorname{Re}\left[S_{\mathrm{s}}(\Omega)\right]
$$

O emaranhamento pode ser testado através dos autovalores simpléticos como mostrado na figura 6.4 . 


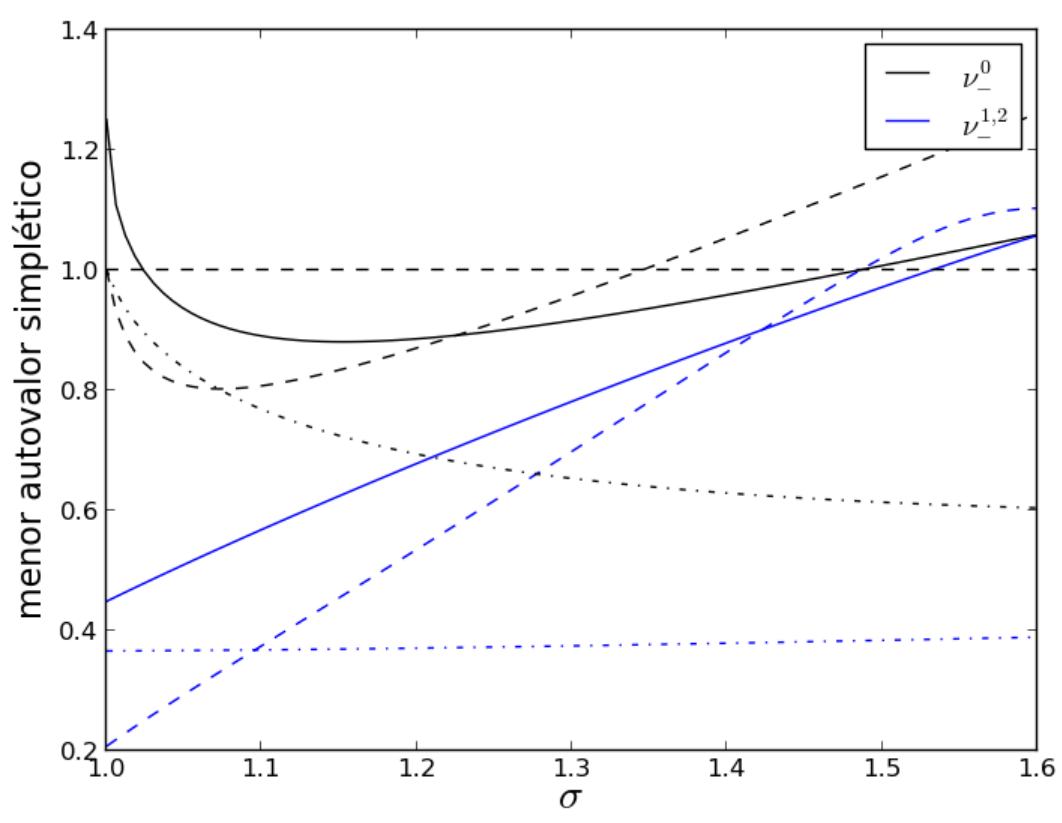

Figura 6.4: Menores autovalores simpléticos calculados a partir de transposições parciais da matriz $V_{+}$para os parâmetros $\gamma_{0}=0.15, \gamma=0.02, \Omega=0.45, \mu_{0}=0.06, \mu=0.002 e$ $\eta=(0.20,0.095,0.105,0.120,0.130,0.060)^{T}$ (curvas cheias) e $\eta=(0,0,0,0,0,0)^{T}$ (curvas pontilhadas). Nas curvas tracejadas, considero $\mu_{0}=0$ e $\mu=0$ na presença do excesso de ruído de fase. Note que devido ao excesso de ruído, perdas maiores podem agir de maneira positiva para o emaranhamento. Nas curvas pretas, o bombeio é transposto e nas curvas em azul, um dos gêmeos é transposto.

\subsubsection{Squeezing de Dois Modos}

Uma forma menos direta de resolver o sistema (6.38), mas que trás um pouco mais de intuição física sobre o sistema é obtida ao passar para o espaço de soma e subtração entre os feixes gêmeos 8

$$
\delta q_{ \pm}=\frac{1}{\sqrt{2}}\left(\delta q_{1} \pm \delta q_{2}\right) \quad \text { e } \quad \delta p_{ \pm}=\frac{1}{\sqrt{2}}\left(\delta p_{1} \pm \delta p_{2}\right)
$$

Neste espaço, as variáveis no subespaço de subtração se desacoplam do resto como pode ser visto em 6.31 6.36). Para dessintonia nula, $\Delta=\Delta_{0}=0$, as equações para o subespaço de subtração se tornam,

$$
\frac{d}{d t} \delta q_{-}=-2 \gamma^{\prime} \delta q_{-}+\sqrt{2 \gamma} v_{-}^{q}+\sqrt{2 \mu} v_{-}^{q \prime} \text { e } \frac{d}{d t} \delta p_{-}=\sqrt{2 \gamma} v_{-}^{p}+\sqrt{2 \mu} v_{-}^{p \prime} .
$$

\footnotetext{
${ }^{8}$ É importante não confundir esta transformação com a de soma e subtração de bandas laterais discutida no capítulo 3 Aquela transformação é realizada entre as bandas laterais inferior e superior de cada campo e ocorre de fato no experimento, pois forma o espaço privilegiado pela fotodetecção. Aqui a passagem para o espaço de soma e subtração é uma conveniência matemática. Não fazemos esta transformação no nosso experimento, as a mesma pode ser implementada de incidimos cada feixe gêmeo em uma das portas de um divisor de feixes balanceado. Note que isto funciona somente se os dois campos possuem portadoras com a mesma frequência.
} 
Se não houvesse cristal na cavidade, não haveria geração de feixes gêmeos e o estado de saída seria o mesmo estado de vácuo de entrada. A equação para a evolução de $\delta q_{-}$e $\delta p_{-}$para esta situação pode ser encontrada fazendo $\chi=0$ em (6.31,6.36), o que faz com que a matriz $M$ seja diagonal e leve ao seguinte conjunto de equações para o subespaço de subtração,

$$
\frac{d}{d t} \delta q_{-}^{v a c}=-\gamma^{\prime} \delta q_{-}^{v a c}+\sqrt{2 \gamma} v_{-}^{q}+\sqrt{2 \mu} v_{-}^{q \prime} \quad \text { e } \frac{d}{d t} \delta p_{-}^{v a c}=-\gamma^{\prime} \delta q_{-}^{v a c}+\sqrt{2 \gamma} v_{-}^{p}+\sqrt{2 \mu} v_{-}^{p \prime} .
$$

Note que aqui a equação para ambas as quadraturas possuem um mesmo termo de decaimento. O decaimento com o fator de $2 \gamma^{\prime}$ em (6.48) é o responsável pelo squeezing na subtração das intensidades no OPO acima do limiar. Como a quadratura conjugada não possui termos de decaimento ela pode, em princípio, se tornar arbitrariamente grande. Para tornar este argumento mais explícito vamos olhar para as soluções das equações (6.49) e (6.48). Todas elas serão da forma

$$
f_{\beta}(t)=\int_{-\infty}^{t} d t_{1} \mathrm{e}^{-\beta \gamma^{\prime}\left(t-t_{1}\right)}\left[\sqrt{2 \gamma} v\left(t_{1}\right)+\sqrt{2 \mu} v^{\prime}\left(t_{1}\right)\right]
$$

em que $\delta q_{-}^{v a c}=\delta p_{-}^{v a c}=f_{1}, \delta q_{-}=f_{2}$ e $\delta p_{-}=f_{0}$. Os termos de vácuo são representados por $v=v_{-}^{q}$ ou $v_{-}^{p}$ e $v^{\prime}=v_{-}^{q}$ ou $v_{-}^{p \prime}$. Os processos de entrada e saída da cavidade levam a um modo de saída de $f_{\beta}^{S}(t)=\sqrt{2 \gamma} f_{\beta}(t)-v(t)$. Portanto, as correlações temporais do campo intracavidade e do campo de saída se tornam

$$
\left\langle f_{\beta}(t) f_{\beta}\left(t^{\prime}\right)\right\rangle=\frac{1}{\beta} \mathrm{e}^{-\beta \gamma^{\prime}\left|t-t^{\prime}\right|} \text { e }\left\langle f_{\beta}^{S}(t) f_{\beta}^{S}\left(t^{\prime}\right)\right\rangle=\delta\left(t-t^{\prime}\right)-2 \gamma\left(1-\frac{1}{\beta}\right) \mathrm{e}^{-\beta \gamma^{\prime}\left|t-t^{\prime}\right|}
$$

pois $\left\langle v_{-}^{q}\left(t_{1}\right) v_{-}^{q}\left(t_{2}\right)\right\rangle=\left\langle v_{-}^{p}\left(t_{1}\right) v_{-}^{p}\left(t_{2}\right)\right\rangle=\delta\left(t_{1}-t_{2}\right)$, sendo que o mesmo vale para $v_{-}^{p}{ }^{\prime}(t)$ e $v_{-}^{q \prime}(t)$ e todas as outras correlações se anulam. Note que estas correlações temporais preveem fotocorrentes estacionárias. Quando $\beta=1$, o estado de saída tem a mesma correlação do estado de vácuo de entrada como era de se esperar. Para $\beta=2$, há uma diminuição da correlação temporal por um fator $\gamma \mathrm{e}^{-2 \gamma^{\prime}\left|t-t^{\prime}\right|}$, o que significa que há squeezing em relação ao ruído de vácuo quando $t=t^{\prime}$. A correlação temporal fora da cavidade diverge além de uma delta de Dirac quando $\beta=0$.

No domínio da frequência, a solução para este sistema é

$$
\begin{aligned}
& \delta q_{-}^{S}(\Omega)=-\left(\frac{2 \gamma}{i \Omega-2 \gamma^{\prime}}+1\right) v_{-}^{q}(\Omega)-\frac{2 \sqrt{\gamma \mu}}{i \Omega-2 \gamma^{\prime}} v_{-}^{q \prime}(\Omega), \\
& \delta p_{-}^{S}(\Omega)=(2 \gamma-1) v_{-}^{p}(\Omega)+2 \sqrt{\gamma \mu} v_{-}^{p}(\Omega) .
\end{aligned}
$$

Através do cálculo dos espectros de ruído podemos prever squeezing em $\delta q_{-}^{S}$ indepen- 


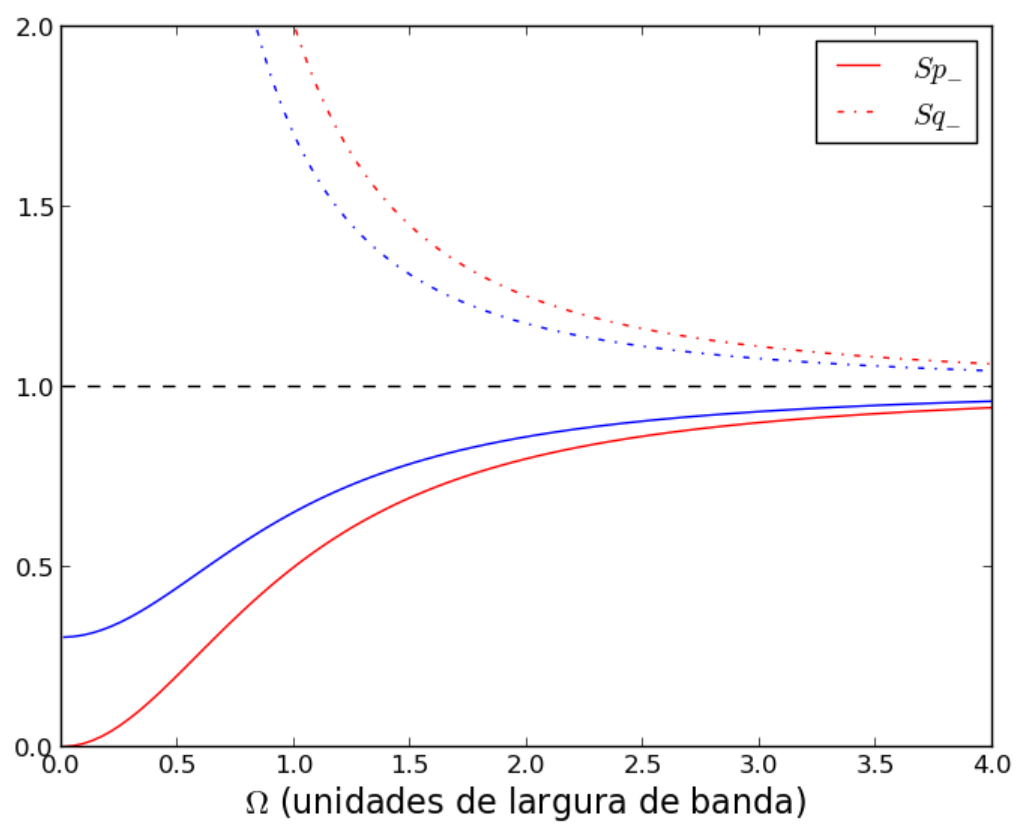

Figura 6.5: Squeezing de intensidade de antisqueezing de fase na subtração das intensidades. As curvas azuis representam a situação em que $\mu=0.1 \gamma$ e as curvas vermelhas em que $\mu=0$.

dente de $\sigma$ para qualquer valor de $\Omega$,

$S q_{-}(\Omega)=\left\langle\delta q_{-}^{S}(\Omega) \delta q_{-}^{S}(-\Omega)\right\rangle=\frac{\gamma^{\prime} \Omega^{2}+\mu}{\gamma^{\prime}\left(1+\Omega^{2}\right)} \quad$ e $\quad S p_{-}(\Omega)=\left\langle\delta p_{-}^{S}(\Omega) \delta p_{-}^{S}(-\Omega)\right\rangle=\frac{\gamma^{\prime} \Omega^{2}+\gamma}{\gamma^{\prime} \Omega^{2}}$.

O ruído de intensidade no modo de subtração é sempre menor que o ruído quântico padrão, o que ocorre devido às correlações de intensidade presentes no OPO. Essa compressão será maior quanto mais próximo da frequência de ressonância estivermos medindo, ou seja, quanto menor $\Omega$. Quando a cavidade é perfeita para os feixes gêmeos, $\mu=0$ e $S q_{-}(\Omega)=1 / S p_{-}(\Omega)=\Omega^{2} /\left(1+\Omega^{2}\right)$, temos um estado comprimido de fato na subtração. Dependendo da frequência de análise, obtemos diferentes valores de squeezing tendo como casos limite o estado de vácuo $\left(S q_{-}(\Omega \rightarrow \infty) \rightarrow 1\right)$ e o estado de squeezing infinito tratado por $\operatorname{EPR}\left(S q_{-}(\Omega \rightarrow 0) \rightarrow 0\right)$ [29]. Note também que o squeezing sofre bastante com as perdas intracavidade. Nestas circunstâncias, $S q_{-}(\Omega \rightarrow 0)=1-\eta$, onde $\eta=\gamma / \gamma^{\prime}$ é a eficiência quântica. Esta relação significa que para perdas espúrias de apenas $10 \%$ do coeficiente de transmissão, $S q_{-}(\Omega \rightarrow 0) \approx 0.1$, o que é equivalente a um fator de squeezing de 1,15 (ou $10 \mathrm{~dB}$ ). 


\subsection{Correlações EPR no OPO}

Como dito anteriormente, o emaranhamento bipartite entre os gêmeos pode ser visto como um sistema do tipo EPR [29], no qual flutuações de intensidade e fase fazem o papel de posição e momento, respectivamente. Como os fótons são emitidos aos pares, os feixes gêmeos possuem correlações de intensidade. O fato da intensidade de um deles diminuir (aumentar) é consequência da presença de menos (mais) fótons neste feixe. Como para cada fóton emitido (absorvido) em um dos feixes gêmeos, outro será emitido (absorvido) no outro, a intensidade de ambos irá variar da mesma forma. Consequentemente, uma flutuação $\delta I_{1}$ em um deles corresponde a um flutuação no outro de mesmo valor,

$$
\delta I_{1}-\delta I_{2}=0
$$

Como explicitado em (2.44), as fases também estão correlacionadas devido à relação de conservação de energia. Como $\omega_{i} \approx \delta \phi_{i} / \delta t$, a expressão 2.10 pode ser escrita como

$$
\delta \phi_{0}=\delta \phi_{1}+\delta \phi_{2}=0
$$

onde na última igualdade considero o bombeio como um campo clássico cuja fase é fixada. Portanto, há uma anticorrelaçãd ${ }^{9}$ para as flutuações de fase entre os feixes gêmeos. As relações 6.53) e 6.52) são as mesmas observadas para as variáveis $E P R, \mathrm{o}$ que dá uma ideia física sobre a origem do emaranhamento.

Quando consideramos o bombeio como um campo quantizado, podemos observar emaranhamento entre este e os gêmeos. Para o emaranhamento tripartite não há uma relação tão próxima com estados bem conhecidos como a que os feixes gêmeos apresentam com os pares EPR. Porém, também podemos entende-lo em termos de correlações entre fase e intensidade. Como a absorção de um fóton do bombeio produz a emissão dos fótons gêmeos, flutuações na soma da intensidade dos feixes gêmeos dão origem a flutuações de sinal oposto na intensidade do bombeio. Este efeito pode ser visto como paralelo à depleção do bombeio, discutida anteriormente, aplicada as flutuações em vez da intensidade média. Entretanto, aqui pode acontecer tanto a depleção quanto o aumento da intensidade do bombeio refletido em torno da intensidade média contanto que a soma das intensidades de sinal e complementar variem de maneira oposta. Esta

\footnotetext{
${ }^{9}$ Aqui me refiro à anticorrelação para explicitar o fato que que uma fase varia em sentido oposto à outra.
} 
anticorrelação de intensidade é resumida como

$$
\delta I_{0}=-\left(\delta I_{1}+\delta I_{2}\right)
$$

Com relação à fase, as flutuações no bombeio estão correlacionadas com a soma das flutuações de fase dos feixes gêmeos pela primeira equação em (6.53). Estas correlações, apresentadas em (6.53) e (6.54), dão origem ao emaranhamento tripartite entre os feixes gêmeos e o bombeio refletido pela cavidade

No próximo capítulo, vou apresentar os resultados experimentais mais importantes conseguidos durante o doutorado. Nele, todos os conceitos desenvolvidos e discutidos em toda a tese serão aplicados à caracterização do estado produzido pelo $O P O$ e ao estudo do emaranhamento e da sua dinâmica frente a perdas. Ao final também discuto um método para testar se os estados que produzimos possuem estatística gaussiana, como previsto pela teoria. 


\section{$7 \quad$ Medidas de Emaranhamento e Desemaranhamento no OPO}

\subsection{Emaranhamento e Robustez no OPO}

Neste capítulo vou apresentar os resultados experimentais obtidos durante o doutorado. Aqui, as ideias discutidas no decorer da tese serão utilizadas para estudar o emaranhamento gerado pelo OPO operando acima do limiar. Como explicitado no capítulo anterior, aplicamos os critérios de emaranhamento na matriz de covariâncias dos operadores de quadratura. Em um primeiro momento, utilizamos a matriz de covariâncias proveniente da parte real da matriz espectral. Esta é uma matriz de covariâncias legítima quando interpretada como sendo a matriz de covariâncias dos operadores de soma entre as bandas laterais inferior e superior de um único campo que chamei no decorrer do texto como uma descrição efetiva de um modo por portadora. No caso de um estado para o qual a parte imaginária da matriz espectral é bem menor que a parte real, como é o caso do nosso OPO, o emaranhamento dentro da descrição de um modo é pouco afetado pela informação a mais introduzida pela parte imaginária.

\subsubsection{Sistema Tripartite}

Como será discutido mais adiante, analisar o estado que produzimos fora desta aproximação de um modo - sistema hexapartite formado pelas duas bandas lateiras dos três campos - permite que separemos subsistemas que possuem mais correlações do que as medidas na aproximação de um modo por portadora. Esta possibilidade de subsistemas mais correlacionados se traduz em maior violação do critério de emaranhamento testado. Se o estado dentro da aproximação de um modo for gaussiano, as entradas desta matriz de covariâncias guardam toda a informação sobre o emaranhamento, pois o critério PPT é necessário e suficiente [102].

A descrição do sistema dentro desta aproximação foi analisada em detalhes pelo 
estudante do grupo Antonio Sales Coelho, que defendeu seu doutorado recentemente. Estas medidas foram realizadas utilizando o OPO 1 como descrito no capítulo 4 . As cavidades de análise utilizadas para a detecção dos ruídos e correlações possuíam largura de banda de 16(1) MHz e finesses por volta de 140(5). Um fator de suma importância para a observação do emaranhamento tripartite foi a diminuição da temperatura do cristal, pois havia um excesso de ruído de fase presente no sistema que impedia a violação dos critérios de emaranhamento. Este excesso de ruído foi caracterizado como efeito elasto-ótico devido ao acoplamento de cada portadora com suas respectivas bandas laterais induzida pelos fônons do cristal [17].

De posse deste resultado, passamos a investigar como o emaranhamento gerado se comportava ao sofrer perdas. $\mathrm{O}$ fato de que o emaranhamento, para sistemas com apenas dois modos, é degradado por perdas de transmissão já era bem conhecido [89]. Inicialmente, a ideia era ter uma estimativa do quanto rápido isto ocorreria em nosso sistema tripartite e não esperávamos que ele se tornasse separável somente pela atenuação dos feixes. Esta expectativa era baseada no fato de que os estados emaranhados tripartites gaussianos, em geral, podem ser vistos como combinações de estados comprimidos em divisores de feixes [113]. Como o fator de compressão somente se anula para atenuação total e a compressão de ruído presente nos campos antes do divisor de feixes é responsável pelo emaranhamento após o mesmo, esperávamos que os nossos estados fossem robustos frente a perdas. Entretanto, vimos que, para algumas potências de bombeio, nossos estados se aproximavam da fronteira com os separáveis quando atenuados. A possibilidade de ocorrer este desemaranhamento frente a perdas possui um paralelo com a chamada morte súbita do emaranhamento (ESD) - do inglês Entanglement Sudden Death [130], já observada experimentalmente em variáveis discretas [131].

O comportamento do emaranhamento, ou qualquer outro recurso, frente a perdas constitui um problema de importância para quaisquer protocolo de informação quântica para o qual o respectivo recurso seja necessário. O emaranhamento possui a complicação adicional que, uma vez degradado, não há como recuperá-lo utilizando somente operações locais e comunicação clássica como é comum se fazer em sistemas de comunicação clássica, nos quais não há limitações fundamentais para o quanto é possível amplificar um sinal. Após ser degradado, o emaranhamento pode, no máximo, ser concentrado a partir do uso de diversas cópias do mesmo estado. Esta ideia é parte fundamental do protocolo de repetidores quânticos que propõe uma solução para a comunicação de informação quântica sobre longas distâncias, como 

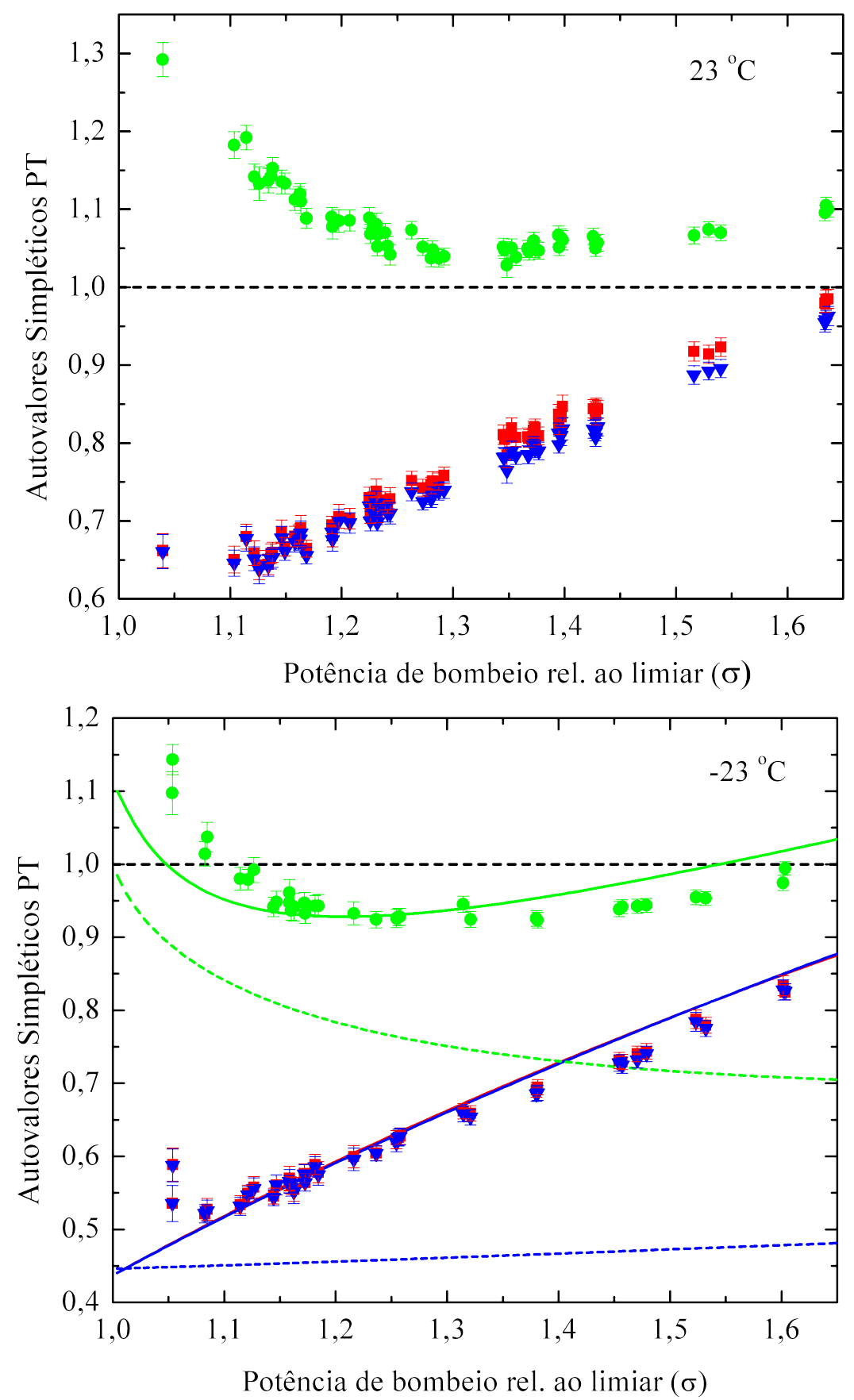

Figura 7.1: Autovalores simpléticos em função da potência de limiar. Os pontos verdes representam a separabilidade do bombeio com o conjunto formado pelos feixes gêmeos. Os pontos azuis e vermelhos representem a separabilidade de cada um do gêmeos com o conjunto composto pelo bombeio e pelo outro feixe gêmeo. As curvas cheias representam a variação do autovalor simplético com $\sigma$ levando em consideração o excesso de ruído de fase e as curvas tracejadas como seria o sistema sem este excesso de ruído. Como discutido no capítulo 5, $v<1$ implica em emaranhamento. Note que o excesso de ruído afeta as três curvas mostradas, mas ele se torna mais importante para o emaranhamento do bombeio com os gêmeos, pois este é naturalmente mais próximo da região separável. 
discutido em [18, 19]. Este protocolo é capaz de concentrar uma quantidade ínfima de emaranhamento presente em cada uma das diversas cópias e produzir uma estado com emaranhamento tão grande quanto se queria, desde que tenhamos cópias suficientes. Entretanto, é necessário que haja emaranhamento em cada uma das cópias. Se o estado perde completamente o emaranhamento devido ao efeito das perdas, o protocolo não funciona. Desta forma, ser capaz de prever se o emaranhamento de um determinado estado é mantido não frente a perdas parciais impõe um limite superior para a distância que este pode ser propagado antes de que o procedimento de concentração ou destilação - seja realizado.

Outro exemplo de protocolo de comunicação quântica no qual o emaranhamento possui um papel fundamental é o de teletransporte [2, 3]. Para este seja bem sucedido, uma das partes que forma um par EPR deve ser transmitida por um canal quântico. Entretanto, para que o teletransporte seja confiável, é necessário que se empregue estados fortemente emaranhados. Deste ponto de vista, a degradação do emaranhamento com as perdas de transmissão se torna um problema também para o teletransporte. Neste caso, o protocolo de repetidores quânticos também, em princípio poderia ser utilizado. Tanto a destilação quanto os repetidores quânticos foram implementados para variáveis discretas utilizando apenas elementos óticos lineares [20, 23]. Para variáveis contínuas, as primeiras implementações experimentais ocorreram recentemente [22, 21] e são necessárias operações gaussianas ou estados não-gaussiano para que ocorram [132, 133, 134]. Neste contexto, se insere nosso trabalho, pois assim como no exemplo citado, os protocolos de comunicação quântica que utilizam a destilação ${ }^{1}$ necessitam que os estados iniciais estejam emaranhados, mesmo que fracamente. Deste ponto de vista, se torna necessário utilizar estados robustos frente a perdas, ou ao menos se ter uma estimativa de quão longe o estado pode ser transmitido sem que o emaranhamento inicial seja completamente destruído.

Para garantir que o estado final seja separável, é preciso aplicar um critério necessário e suficiente para o emaranhamento. Se o critério utilizado for somente suficiente ainda restaria a dúvida se o sistema realmente deixou de ser emaranhado ou apenas evoluiu para um estado cujo emaranhamento não é detectado pelo mesmo. Utilizamos o critério $P P T$, que é necessário e suficiente para estados gaussianos com bipartições do tipo $(1 \otimes N)$ [102]. Em teoria, o estado tripartite gerado em nosso sistema é gaussiano de acordo com as aproximações que fazemos. Um estudo detalhado sobre a gaussianidade analisada à luz dos momentos de ordem superior foi apresentada na

\footnotetext{
${ }^{1}$ E grande parte dos protocolos em comunicação quântica são baseados nesta ideia.
} 

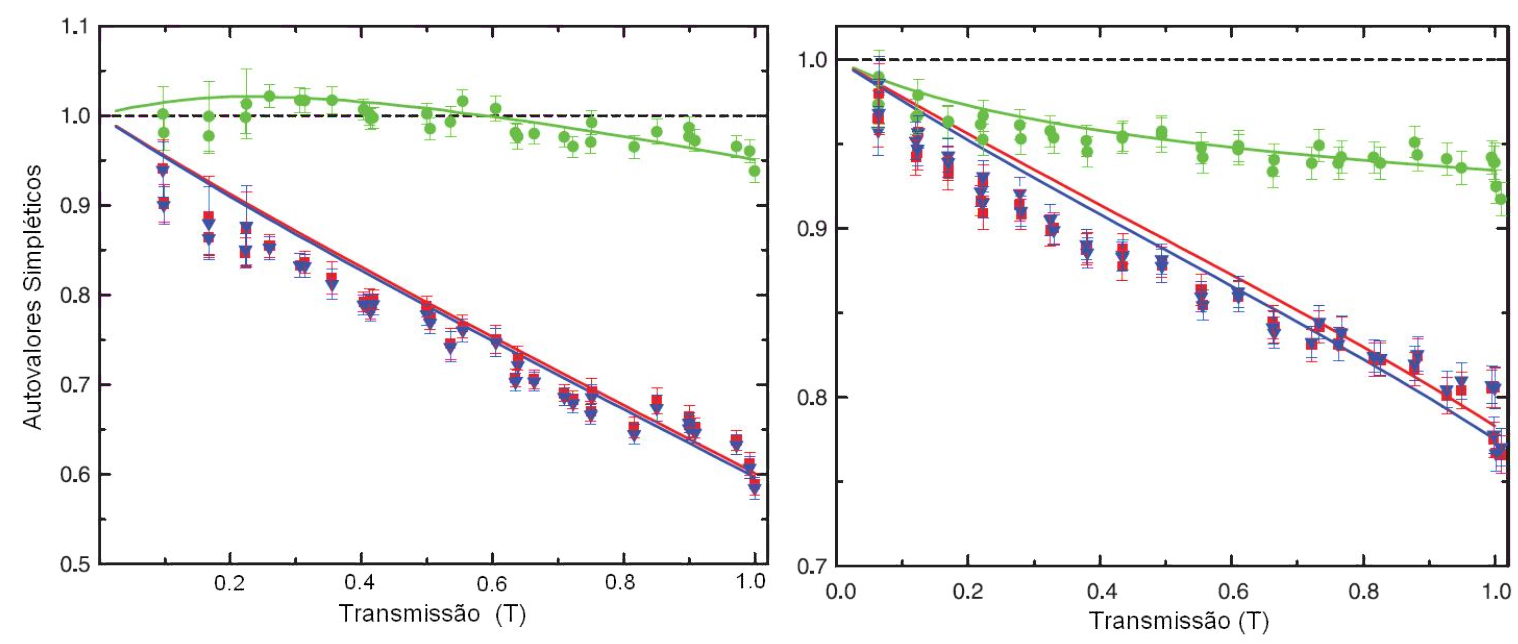

Figura 7.2: Comportamento dos autovalores simpléticos em função da atenuação. Note que o emaranhamento entre um dos feixes gêmeos e o conjunto bombeio+gêmeo persiste até atenuação total, mas há regiões em que o bombeio se torna separável dos gêmeos. Isto ocorre bem próximo do limiar. Entretanto, para potências da ordem de $40 \%$ acima do limiar, o emaranhamento é robusto. A potência relativa ao limiar é $\sigma=1,40$ e 1,18 para as figuras à direita e à esquerda, respectivamente.

tese de Antonio Sales Coelho. Mais adiante, irei analisar este problema do ponto de vista de teste de hipóteses.

Realizamos as medidas de atenuação para diversas de intensidade e encontramos regiões nas quais o emaranhamento é robusto frente a perdas e outras em que o sistema se torna separável para perdas parciais. Duas destas regiões, uma 15\% acima do limiar e outra $40 \%$ acima do limiar, são mostradas na figura 7.2. No experimento, aplicamos perdas balanceadas somente nos feixes gêmeos utilizando combinações de lâmina de meia-onda e divisores de feixes polarizantes. As medidas foram feitas com o cristal de $\mathrm{KTP}$ resfriado a $-10^{\circ} \mathrm{C}$ e para várias potências de bombeio. Os autovalores dos simpléticos em função da atenuação para as melhores dessas medidas, que foram publicadas em [26] são mostrados na figura 7.2.

\subsubsection{Sistema Bipartite formado pelos Feixes Gêmeos}

Devido a maior complexidade do sistema tripartite, resolvemos investigar a dinâmica frente a perdas do sistema formado pelos feixes gêmeos, cujo emaranhamento já havia sido experimentalmente demonstrado em nosso grupo [13]. Realizamos paralelamente estudos teóricos, como descrito no capítulo 5, e experimentais sobre a robustez do emaranhamento neste sistema. Fizemos medidas de emaranhamento em função da atenuação para o sistema bipartite formado pelos feixes gêmeos gerados pelo $O P O 1$, 
como descrito no capítulo 4 . Observamos que para algumas potências do campo de bombeio, o sistema se tornava separável para perdas parciais.

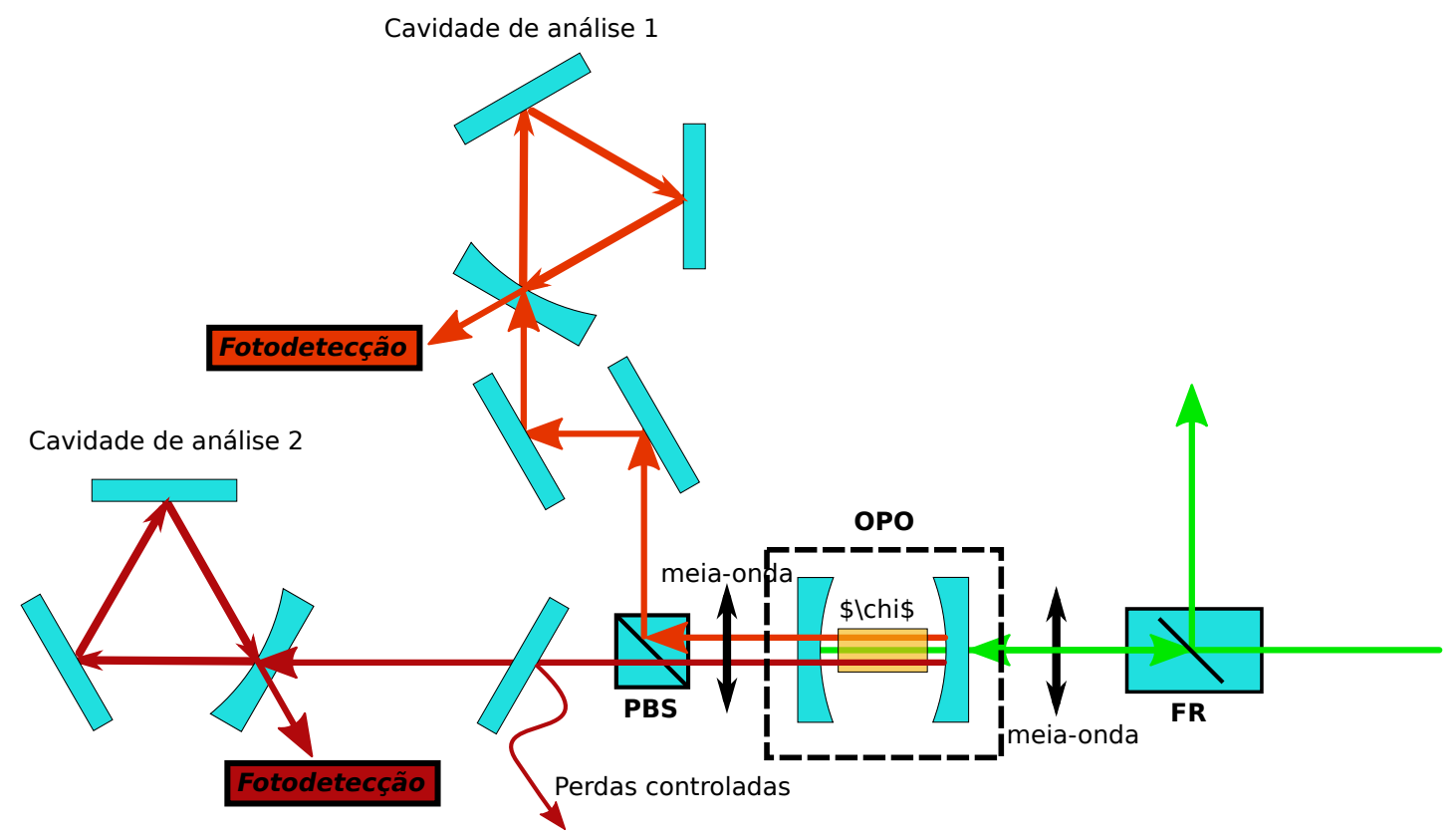

Figura 7.3: Esquema da atenuação utilizado no experimento. Atenuamos somente um dos feixes gêmeos, pois queriamos ser capazes de observar o desemaranhamento mesmo com a atenuação mais simples possível.

Para obter este resultado, utilizamos o segundo sistema de detecção discutido no capítulo 4, pois era necessário uma melhora calibração do shot noise. Como este sistema é mais simples que o tripartite, pudemos estudá-lo teoricamente de forma mais profunda e extrair os elementos responsáveis pelo desemaranhamento. Como visto nas seções anteriores, o desemaranhamento ocorre quando os excessos de ruído, $\delta_{1}$ e $\delta_{2}$, são grandes comparados com a diferença entre as correlações, $\left|c_{p}-c_{q}\right|$. Deste modo, qualquer processo que introduza ruído, mas que favoreça um aumento menor da correlação propicia a geração de estados de emaranhamento frágil. No nosso OPO, o processo por trás disto é o excesso de ruído gerado pelos fônons. Se o OPO fosse descrito satisfatoriamente sem considerar a presença deste ruído, o emaranhamento seria sempre robusto.

Os fônons introduzem tanto ruído quanto correlações de fase nos campos de saída. Porém estas correlações não são perfeitas. De fato, os valores que $\eta_{i j}$ assumem, $\eta_{11} \approx$ $\eta_{22}=0.15$ e $\eta_{12}=0.08$, estão longe de corresponder a uma correlação perfeita, $\eta_{12}^{2} \approx$ $\eta_{11} \eta_{22} / 4$. Como este efeito é maior quanto mais potência usarmos para bombear o $O P O$, estados bem próximos no limiar de oscilação possuem emaranhamento robusto. No nosso OPO, a ESD somente ocorre para estados com potência bem acima do limiar, 

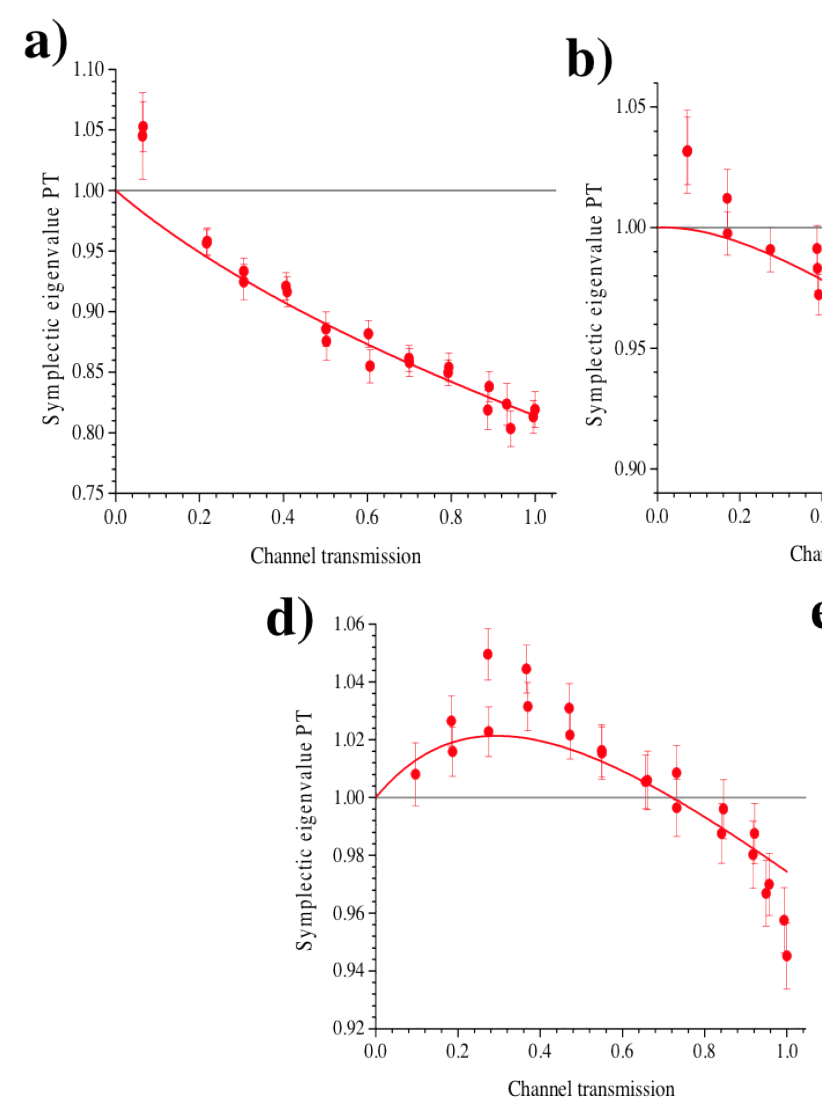

b)

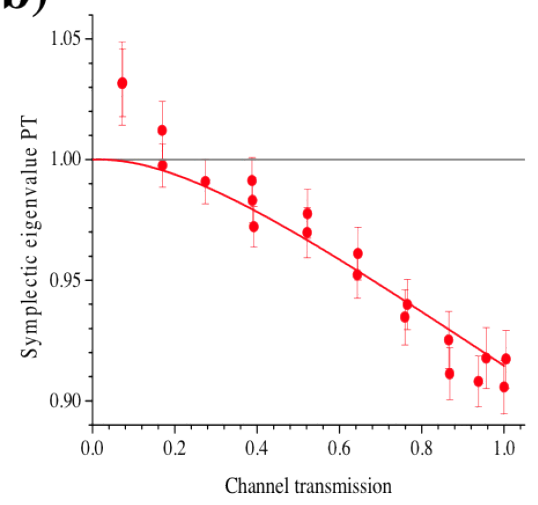

e) c)

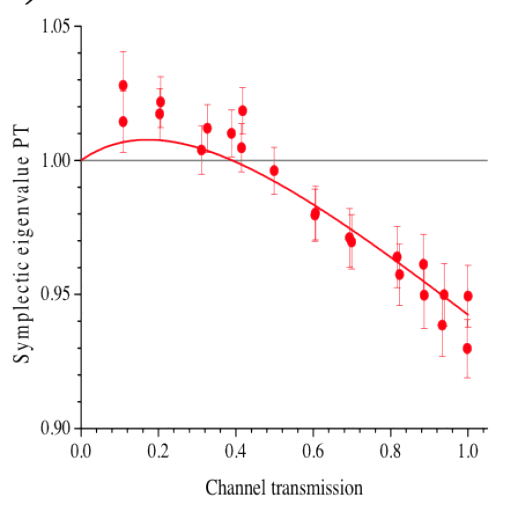

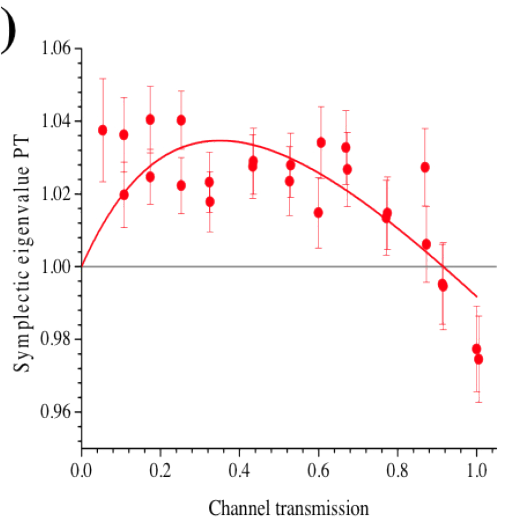

Figura 7.4: Desemaranhamento no sistema bipartite. Diferentemente do sistema tripartite, a ESD somente ocorre para estados bem acima do limiar de oscilação. Muito próximo da potência de limiar, $\Delta_{q+} \approx 1$ fazendo com que, como $\Delta^{2} p_{-} \approx 0.5$ para qualquer potência, o estado seja robusto.

como pode ser visto na figura 7.4

Neste experimento, atenuamos apenas um dos feixes, pois queríamos saber se mesmo a atenuação mais simples resultava em ESD. Os autovalores simpléticos em função da atenuação para a medida em que o desemaranhamento ocorre de forma mais marcante são mostrados na figura 7.4 para diversas potências de bombeio [27].

\subsection{Descrição do OPO nas Bandas Laterais}

Como discutido ao longo do capítulo 3, observamos diretamente dois modos de cada campo quando fazemos a demodulação. Desta forma, o estado gerado pelo OPO é formado por 6 modos. Entretanto podemos tratar o estado gerado pelo OPO como sendo formado por um conjunto de 3 modos efetivos que representam os operadores de quadratura no subespaço de soma (ou subtração) de bandas laterais. A matriz de covariâncias destes modos efetivos é dada pela parte real da matriz de covariâncias 
espectral. Quando a parte imaginária da mesma não possui valor apreciável, não estamos perdendo informação neste processo. Porém, mesmo nesta situação, perdemos "espaço de possibilidades" para explorar o emaranhamento, pois estamos reduzindo a complexidade do sistema. Quando consideramos o sistema global composto por 6 modos, podemos separar subsistemas mais correlacionados do que com os 3 modos efetivos mostrados até aqui.

Para o OPO não-degenerado, as portadoras do bombeio e de cada um dos feixes gêmeos oscilam com frequência $\omega_{0}, \omega_{1}$ e $\omega_{2}$. Quando o OPO estiver em ressonância, irá ocorrer conversão paramétrica preferencialmente entre as portadoras de modo que a relação de conservação de energia seja satisfeita, $\omega_{0}=\omega_{1}+\omega_{2}$. Porém, pode ocorrer também conversão paramétrica entre outras frequências que estejam dentro da largura de banda da cavidade. Se o cristal absorve um fóton do bombeio e emite um fóton em uma frequência um pouco diferente da de um dos feixes gêmeos, $\omega_{1} \pm \Omega$, o seu fóton gêmeo deverá possuir frequência de $\omega_{2} \mp \Omega$, onde $\Omega$ é menor que a largura de banda da cavidade e maior que a largura de linha de todas as portadoras,

$$
\omega_{0}=\omega_{1} \pm \Omega+\omega_{2} \mp \Omega \text {. }
$$
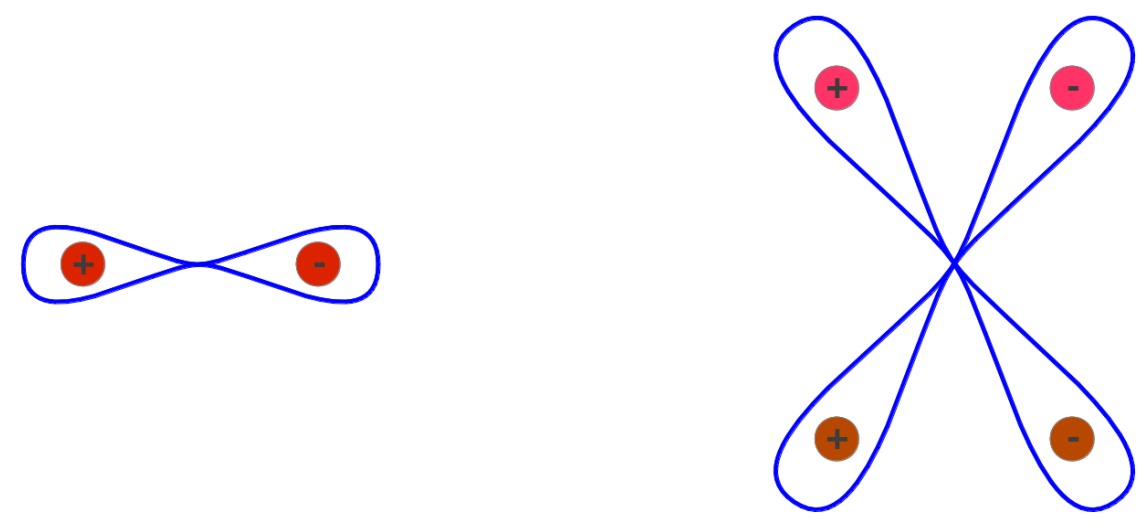

Figura 7.5: Correlações presentes entre a bandas laterais: os círculos com os sinais $+e-$ representam as bandas laterais positiva e negativa, respectivamente. Os laços azuis representam as correlações existentes no sistema. Figura da esquerda: OPO degenerado - correlações entre bandas laterais positiva e negativa do mesmo campo. Figura da direita: OPO não degenerado a cor ligeiramente diferente dos círculos significa que as frequências portadoras são diferentes. Neste caso, há correlações entre a bandas laterais positiva e negativa de campos distintos.

Em outras palavras, a banda lateral negativa de um dos gêmeos estará correlacio- 
nada com a banda lateral positiva do outro e vice-versa. Estes modos oscilam de forma não-ressonante quando as portadoras estão em ressonância. As intensidades dos mesmos, apesar de muito pequenas, estão correlacionadas, pois os fótons são criados aos pares. Estas correlações fazem com que os modos $\omega_{1} \pm \Omega$ e $\omega_{2} \mp \Omega$ não possam mais ser escritos de forma separada. Note que as frequências $\omega_{1} \mp \Omega$ e $\omega_{2} \pm \Omega^{\prime}, \operatorname{com} \Omega \neq \Omega^{\prime}$, não são acopladas, pois a conversão paramétrica depende, entre outras coisas, da intensidade média do campo de bombeio, que não é desprezível apenas para a portadora. Olhando apenas para as bandas laterais, a presença da portadora do bombeio permite a presença de dois sistema descorrelacionados entre si, mas que possuem correlações internas. Os modos de frequência $\omega_{1}+\Omega$ e $\omega_{2}-\Omega$ estão correlacionados, assim como $\omega_{1}-\Omega$ e $\omega_{2}+\Omega$, porém as duas duplas podem ser descritas de forma independente.

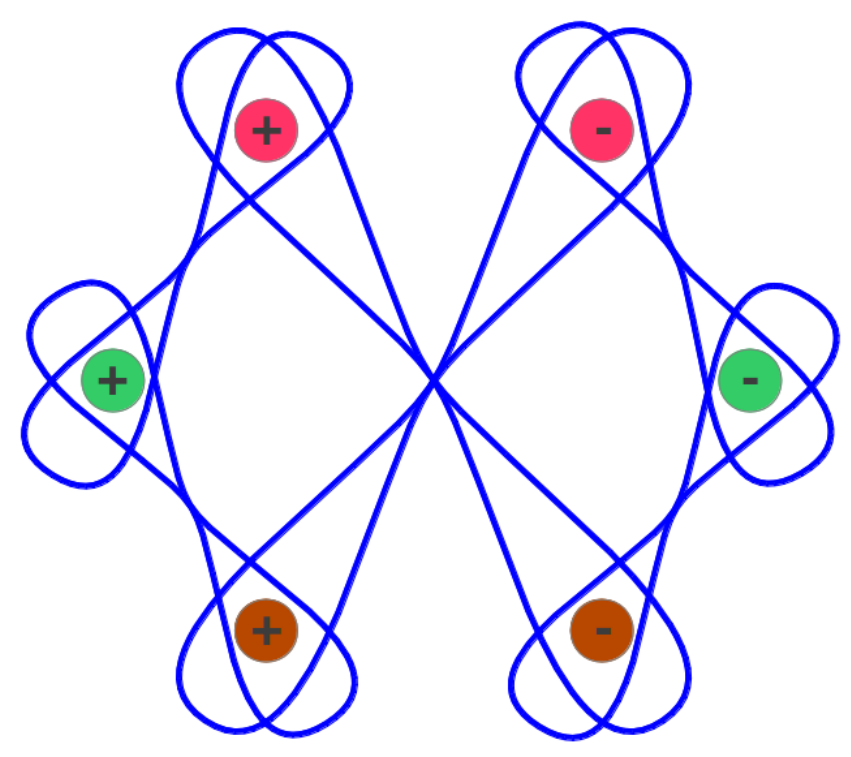

Figura 7.6: OPO triplamente ressonante: os círculos verdes representam o campo de bombeio $\left(0_{+} e 0_{-}\right)$e os círculos vermelhos e laranja representam os feixes gêmeos $\left(1_{+}, 1_{-}, 2_{+} e 2_{-}\right)$. Além das correlações existentes entre os feixes gêmeos, as bandas laterais do bombeio também estão correlacionadas às dos gêmeos de mesmo sinal. Isto faz com que todo o sistema de seis modos não possa ser descrito de forma independente.

Se a cavidade também é ressonante para a portadora do bombeio, suas bandas laterais oscilam fora de ressonância. Neste caso, pode ocorrer a absorção de fótons na portadora de um dos gêmeos e na banda lateral do outro, seguida pela emissão de um 
fóton na banda lateral do bombeio, de tal forma que haja conservação de energia,

$$
\omega_{0} \pm \Omega=\omega_{1}+\omega_{2} \pm \Omega
$$

Consequentemente, a banda lateral superior (inferior) do bombeio está correlacionada com as bandas laterais superiores (inferiores) de ambos os gêmeos. No caso em que os três campos são ressonantes com a cavidade, os seis modos de frequências $\omega_{0} \pm \Omega$, $\omega_{1} \pm \Omega$ e $\omega_{2} \pm \Omega$ estão correlacionados entre si. Cada banda lateral, e suas respectivas correlações, é representada na figura 7.6 possui espaço de fase como descrito no capítulo 3. Por uma questão de simplicidade, representarei cada uma delas como $n_{ \pm}$de modo que $0_{+}\left(0_{-}\right)$represente a banda lateral superior (inferior) do campo de bombeio e o mesmo vale para $1_{ \pm}$e $2_{ \pm}$em relação aos feixes gêmeos.

\subsection{Medidas da Matriz Espectral Complexa}

Como discutido no capítulo 4 , os estados que geramos são consistentes com uma estatística gaussiana. Por este motivo, focaremos nossa atenção apenas nos segundos momentos da fotocorrente. Para isto, os dados brutos são divididos em pacotes de 1000 pontos que são usados para calcular os segundos momentos ${ }^{2}$. Como cada aquisição é formada por um conjunto de 450000 pontos, adquiridos com uma taxa de $1.67 \mu$ s, cada curva de ruído ou correlação espectral conterá 450 pontos. Para fazer a conversão de fase em intensidade, utilizamos três cavidades de análises construídas de maneira idêntica. Elas tem, aproximadamente, $16.7 \pm 0.5 \mathrm{~cm}$ de comprimento e $120 \pm 5$ de Finesse, o que faz com que seus FSRs e larguras de banda sejam de $1.79 \pm 0.05 \mathrm{GHz}$ e $15 \pm 1 \mathrm{MHz}$, respectivamente. Para as medidas apresentadas, escolhemos a frequência de análise de $21 \mathrm{MHz}$, portanto, como $\Omega^{\prime}=1.4 \approx \sqrt{2}$, a cavidade está na situação limite em que consegue separa entre a rotação da portadora e de cada banda lateral.

De posse dos ruídos e correlações entre as componentes seno e cosseno da fotocorrente demodulada, podemos extrair a matriz de covariâncias espectral e, a partir da mesma, inferir as entradas da matriz de covariâncias das bandas laterais. A hipótese de estacionariedade da fotocorrente também pode ser testada diretamente a partir dos ruídos e correlações das componentes seno e cosseno, como discutido no capítulo 3 . Entretanto, esta estacionariedade pode ser devida à falta de controle sobre a diferença de fase entre os osciladores locais ótico e eletrônico também como discutido no capítulo

\footnotetext{
${ }^{2}$ Esta quantidade de pontos é arbitrária e somente foi escolhida pois possui a melhor relação sinal/ruído.
} 

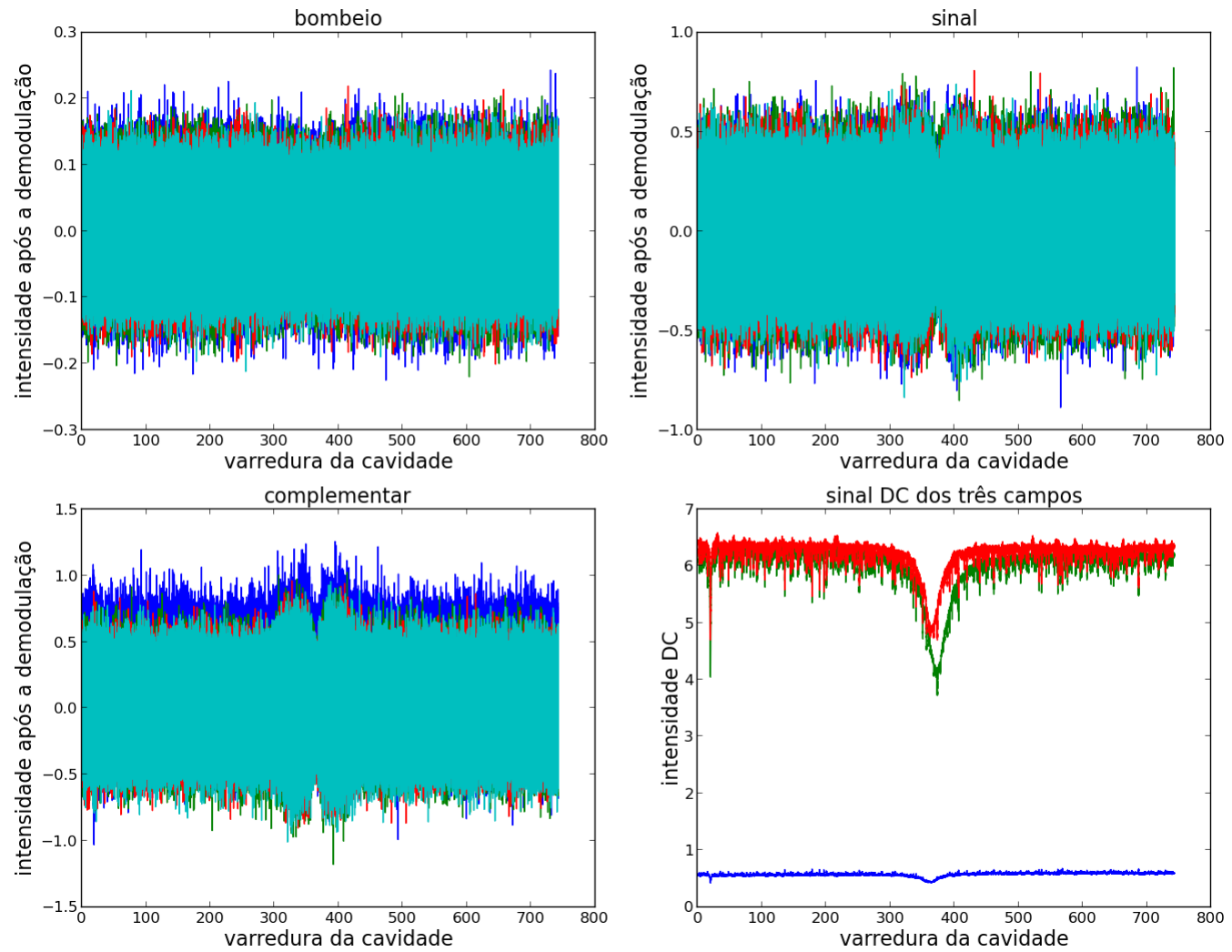

Figura 7.7: Exemplo de um arquivo de dados brutos adquiridos à medida que a cavidade de análise é varrida. Para cada campo, são adquiridos 4 sinais HF (2 detectores +2 osciladores locais eletrônicos) e 1 sinai DC.
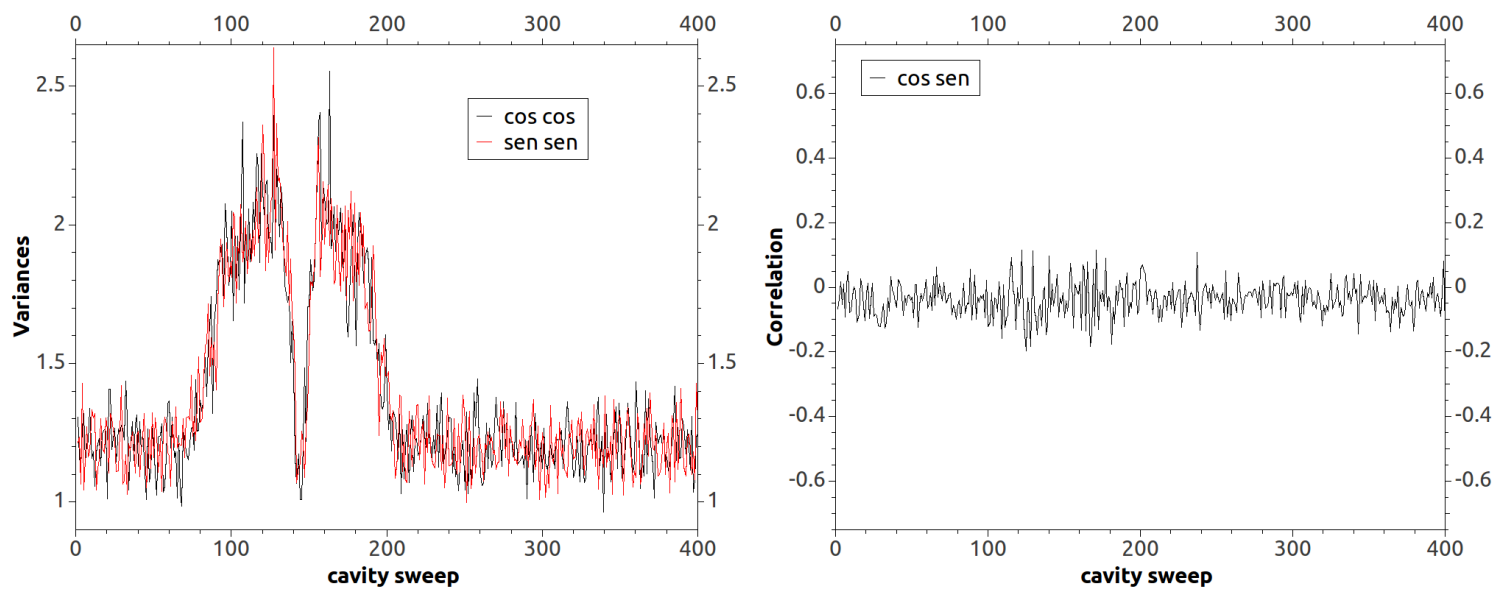

Figura 7.8: Nas curvas da esquerda, podemos ver os ruídos das componentes seno (em vermelho) e cosseno (em preto) da fotocorrente obtidas após a demodulação de um dos feixes gêmeos refletidos por sua respectiva cavidade de análise. Note que ambas as curvas são iguais a menos de flutuações, indicando que a fotocorrente é estacionária. Na curva da direita está a correlação entre as componentes seno e cosseno, que deve se anular para uma fotocorrente estacionária. 

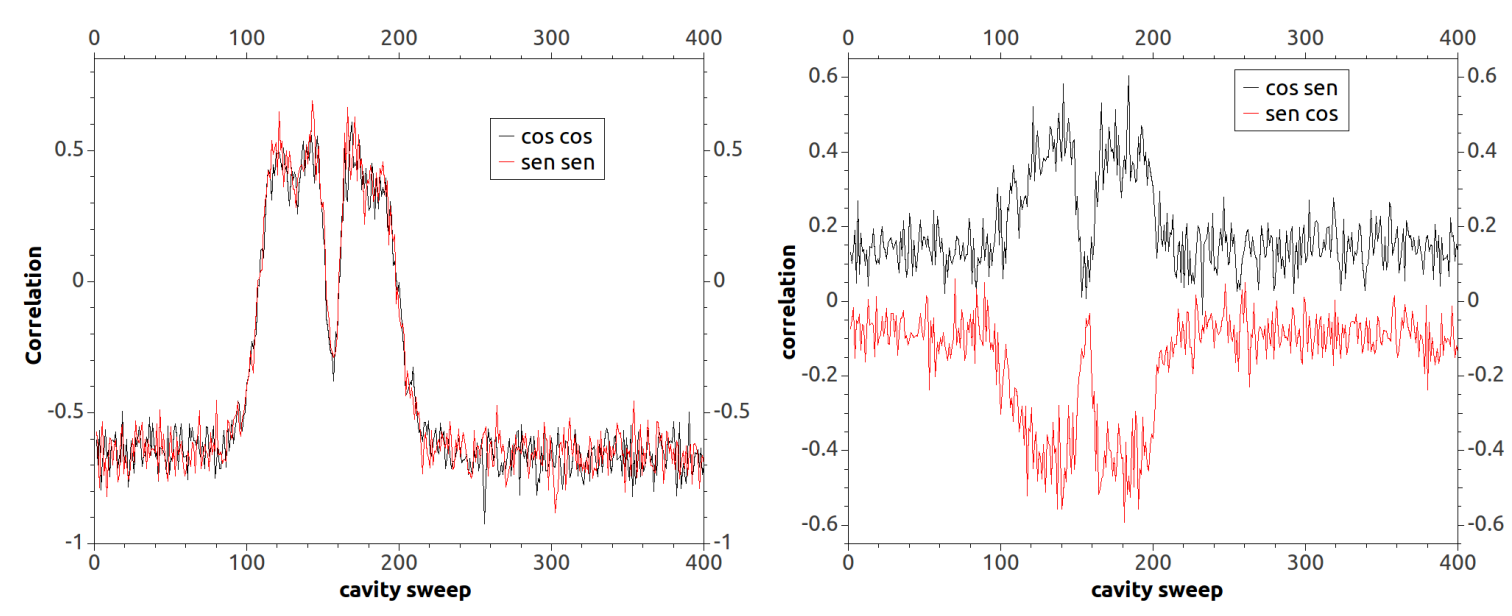

Figura 7.9: Nestas curvas são mostradas as correlações espectrais entre o bombeio e um dos feixes gêmeos. Nas cuvas da esquer estão os ruídos da fotocorrente demodulada com as componentes seno e cosseno Nas curvas da direita, estão as correlações entre as componentes seno e cosseno, que possuem sinais opostos a menos de flutuações. Ambas as curvas mostram que a nossa fotocorrente é estacionária.

3. Os sinais de ruído de correlação mostrados em 7.8 foram medidos a partir do campo gerado pelo OPO 1, descrito no capítulo 4. Como será mostrado na próxima seção, podemos utilizar este sinal para inferir a matriz de covariâncias dos operadores de quadratura do campo de entrada, como discutido no capítulo 3 .

\subsubsection{Ajustes dos Ruídos e Correlações Espectrais}

As componentes das matrizes de covariâncias do campo que entra na cavidade são estimadas a partir de um ajuste de mínimos quadrados. Como discutido nos capítulos 2 e 3, modelamos tanto o sinal DC quanto os HF por meio das equações (3.113) e (2.28). Como mostrado em (2.28), o coeficiente de reflexão da cavidade depende de 3 parâmetros: o seu valor na ressonância $\left(R_{\min }\right)$, a frequência de ressonância $\left(f_{0}\right)$ e a largura de banda da cavidade $(\delta f)$. O sinal DC depende de um parâmetro adicional que é a intensidade fora da ressonância. As curvas 3.113 dependem, além das componentes da matriz de covariâncias, dos três parâmetros do DC $\left(f_{0}, R_{\min }\right.$ e $\left.\delta f\right)$ de cada cavidade e a frequência de análise medida em unidades de largura de banda, $\Omega^{\prime}$ Note que apenas a intensidade média é o único parâmetro que influencia somente as curvas do DC. Por este motivo e para reduzir os erros, o ajuste é feito em todas as curvas de DC e HF simultaneamente. Este procedimento é utilizado para inferir, além dos parâmetros físicos das cavidades de análise $\left(f_{0}, R_{\text {min }}, \delta f\right)$, todas as componentes da 
Medida 16.dat
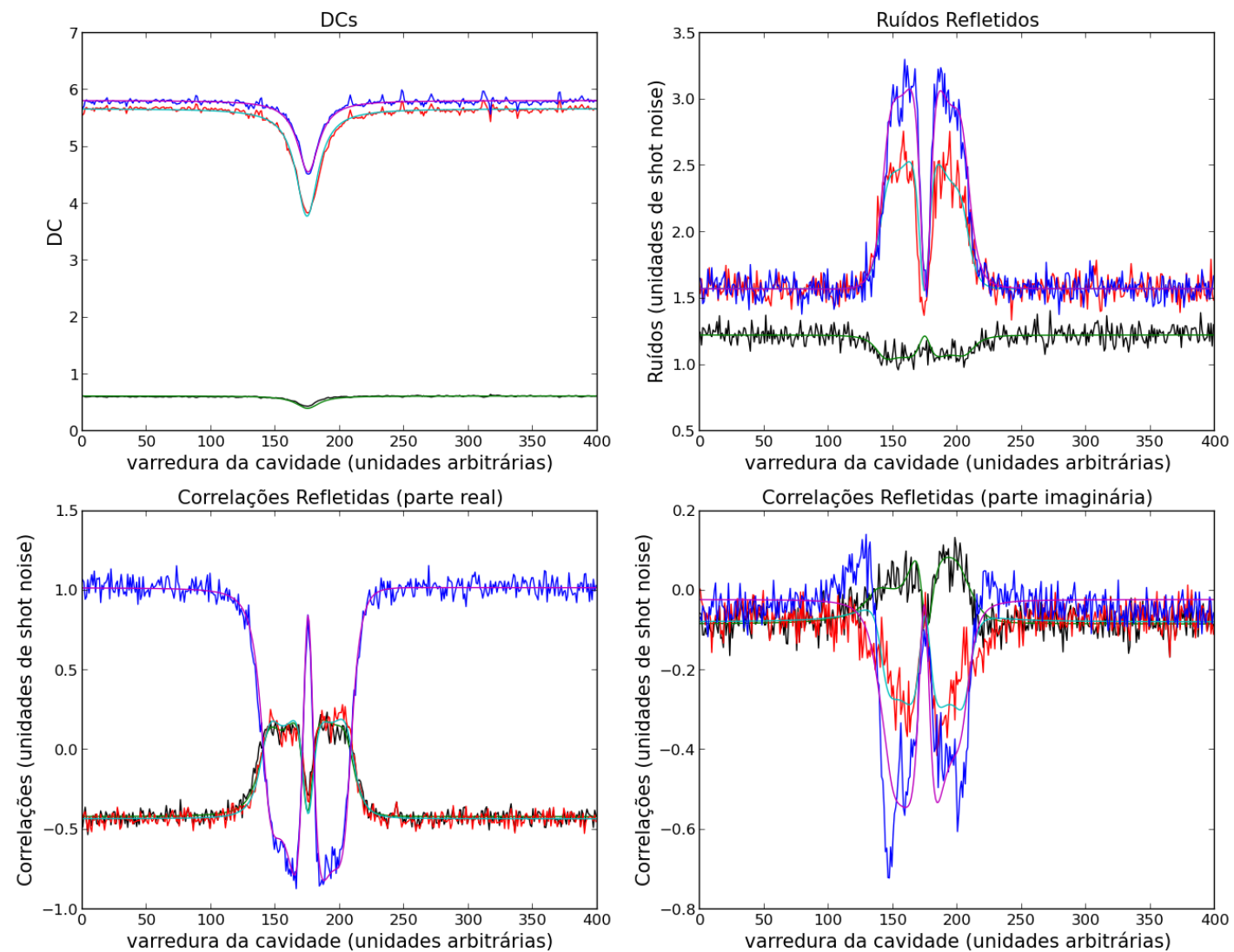

Figura 7.10: Nesta figura são mostradas os ajustes do DC, dos ruídos e das partes real e imaginária das correlações espectrais. Para fazer esta medida, as cavidades de análise foram mantidas em sincronia, de modo que as frequências de ressonância das três coincidissem durante a aquisição. O eixo x neste gráfico representa a varredura das cavidades através da ressonância e está em unidades arbitrárias

matriz de covariâncias do campo de entrada.

\subsubsection{Estimativa das Barras de Erro}

Utilizamos um procedimento de mínimos quadrados para fazer os ajustes. Este procedimento busca a curva que melhor se ajusta aos pontos minimizando a seguinte quantidade,

$$
Q(\beta)=\frac{1}{n \sigma^{2}} \sum_{k=1}^{n}\left[y_{i}-f\left(x_{i} ; \beta\right)\right]^{2}
$$

onde $n$ é o número de pontos para o ajuste, $\beta=\left(\beta_{1}, \ldots, \beta_{m}\right)^{T}$ são os parâmetros que desejamos inferir - no nosso caso, as entradas da matriz de covariâncias -, $y_{i}$ são as 

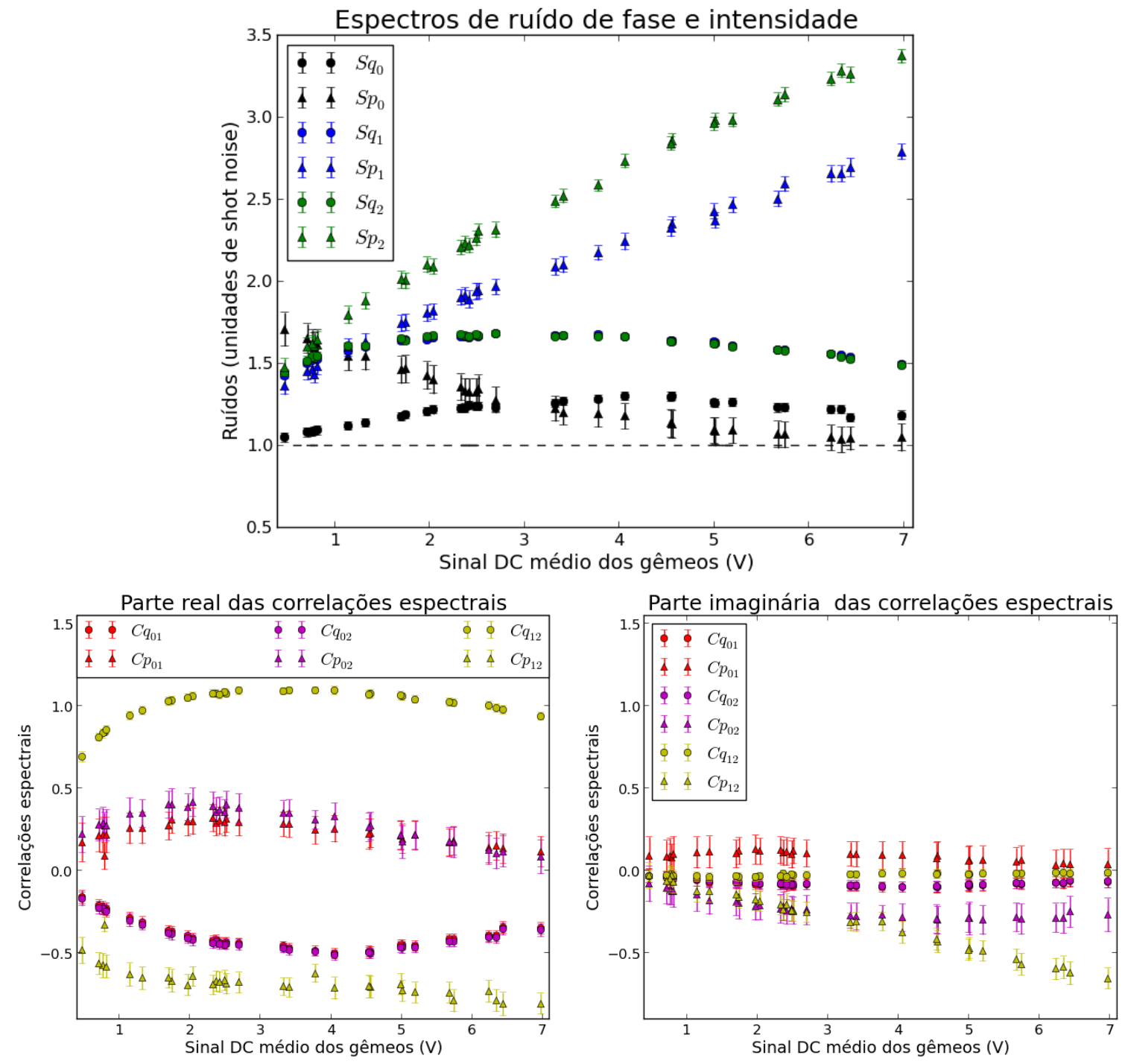

Figura 7.11: Componentes da matriz de covariâncias em função da potência média dos feixes gêmeos. Note que a forma dos ruídos e correlações concordam com o que é mostrado na figura 6.3. tendo em mente que um $D C$ de $7 \mathrm{~V}$ corresponde a uma potência pouco menor que o dobro da potência de limiar. Como discutido no capítulo 6. quando ambos os feixes gêmeos estão em ressonância e possuem as mesmas perdas intracavidade, suas correlações não deveriam possuir parte imaginária, o que não ocorre nestas medidas. Ainda não sabemos o que introduz esta parte imaginária, mas especulamos que ela poderia vir de alguma dessintonia entre a cavidade e os gêmeos ou do excesso de ruído de fase introduzido pelo cristal.

medidas da variável dependente - no nosso caso, os sinais DC, ruídos e correlações e $x_{i}$ re-presenta a variável independente - no nosso caso, a varredura das cavidades. As funções $f\left(x_{i} ; \beta\right)$ são dadas em (3.113) e relacionam o ruído de intensidade do campo refletido pela cavidade de análise com a matriz de covariâncias do campo incidente e a quantidade $\sigma^{2}$ é a variância da variável aleatória associada a cada $y_{i}$ e será explicada mais adiante. Ela é introduzida aqui por mera conveniência. A equação de estimação 
para este sistema é

$$
\left.\frac{\partial Q}{\partial \beta_{i}}\right)_{\beta=\beta_{o t}}=U_{i}\left(\beta_{o t}\right)=0
$$

e pode ser resolvida diretamente pelo cálculo das derivadas ou por métodos numéricos, como o Levenberg-Marquardt, que é o que utilizamos. Temos uma incerteza intrínseca associada às medidas $y_{i}$, pois, como pode ser visto na figura 7.10, há uma dispersão em torno da curva de ajuste. Esta incerteza pode ser usada para estimar a incerteza nos parâmetros $\beta$ [135].

Para que a equação de estimação nos forneça, além das curvas de ajuste, as barras de erro dos parâmetros $\beta$, é necessário associar cada medida $y_{i}$ e cada $\beta_{j}$ - este é chamado de estimador [135] - a variáveis aleatórias. Como o modelo que relaciona cada medida $y_{i}$ a $x_{i}$ é dado por (3.113), então o modelo para variável aleatória $y_{i}$ correspondente a $y_{i}$ será

$$
\mathrm{y}_{i}=f\left(x_{i} ; \beta\right)+e_{i}, \quad \text { onde } \quad\left\langle e_{i}\right\rangle=0 \quad \text { e }\left\langle e_{i} e_{j}\right\rangle=\sigma^{2} \delta_{i j} .
$$

Neste sentido, a ideia básica por trás do procedimento de estimação é inverter a relação acima e o cálculo das barras de erro se torna uma propagação de erro às avessas. Aqui fica mais claro o papel de $\sigma^{2}$ em $(7.3)$, pois desta forma, o valor médio de $Q(\beta)$ não diverge e $\langle Q(\beta)\rangle=1$. Considere também que o procedimento de ajuste vai convergir para um $\beta_{o t}$ e que $\langle\beta\rangle=\beta_{o t}$. Podemos expandir (7.4) em torno da média (ou de $\beta_{o t}$ ) de modo que

$$
U_{j}(\beta)=U_{j}(\langle\beta\rangle)+\sum_{i} \frac{\partial U_{j}}{\partial \beta_{i}} \delta \beta_{i} \Longrightarrow \delta \beta_{i}=\sum_{j} \Gamma_{i j}^{-1} U_{j}(\beta) \quad \text { onde } \quad \Gamma_{i j}=\frac{\partial U_{j}}{\partial \beta_{i}},
$$

$\delta \beta_{i}=\beta_{i}-\left\langle\beta_{i}\right\rangle$ e $U_{j}(\langle\beta\rangle)=0$, pois $\langle\beta\rangle=\beta_{o t}$. Portanto, em notação matricial,

$$
\left\langle\delta \beta(\delta \beta)^{T}\right\rangle=\Gamma^{-1} V^{Q}\left(\Gamma^{T}\right)^{-1}, \quad \text { onde } \quad V_{k l}^{Q}=\left\langle\frac{\partial Q}{\partial \beta_{k}} \frac{\partial Q}{\partial \beta_{l}}\right\rangle .
$$

Os elementos diagonais da matriz de covariâncias dos parâmetros $\beta,\left\langle\delta \beta(\delta \beta)^{T}\right\rangle$, nos fornecerão as barras de erro. Como no nosso caso o método dos mínimos quadrados é linear para as componentes da matriz de covariâncias espectral, pois o modelo é da forma (3.113), as quantidades mostradas na equação anterior serão

$$
\Gamma_{i k}=\frac{2}{n \sigma^{2}} \sum_{p=1}^{n} J_{p i} J_{p k} \quad \text { e } \quad U_{k}=-\frac{2}{n \sigma^{2}} \sum_{p=1}^{n} e_{p} J_{p k} \Rightarrow V_{k l}^{Q}=\frac{4}{n \sigma^{2}} \sum_{p=1}^{n} J_{p k} J_{p l}
$$

onde $\left.J_{p i}=\partial f\left(x_{p} ; \beta\right) / \partial \beta_{i}\right)_{\beta=\beta_{o t}}$ é o Jacobiano. Consequentemente, a matriz de covariâncias 
dos parâmetros inferidos $\beta$ é igual a

$$
V(\beta)=\left\langle\delta \beta(\delta \beta)^{T}\right\rangle=\left(J J^{T}\right)^{-1} \text { res, onde res }=\sum_{i=1}^{n}\left[y_{i}-f\left(x_{i} ; \beta_{o t}\right)\right]^{2}
$$

é um estimador para $n \sigma^{2}$ e é chamado de resíduo. As barras de erro de $\beta$ são dadas pelos elementos diagonais da matriz $V(\beta)$.

É importante ressaltar e que somente hipóteses acerca da média e da variâncias dos $e_{i}$ são necessárias. Em um caso mais específico, poderíamos assumir também que todos os $e_{i}$ são gaussianos. Esta hipótese adicional faz com que o método dos mínimos quadrados se torne um caso particular do método da máxima verossimilhança [135], pois, para uma distribuição gaussiana, a verossimilhança é

$$
L(\beta \mid y)=p_{\beta}(y)=A_{n} \prod_{i=1}^{n} \mathrm{e}^{\frac{\left[y_{i}-f\left(x_{i} ; \beta\right)\right]^{2}}{2 \sigma^{2}}}, \quad \text { portanto, } \quad \ln [L(\beta \mid y)]=Q(\beta) .
$$

onde $A_{n}$ é uma constante de normalização. Uma consequência importante de fazer esta hipótese adicional é que o estimador de máxima verossimilhança possui o menor erro possível dentre todos os estimadores não-enviesados para o mesmo modelo. Em outras palavras, ele é o estimador que satura a desigualdade de Cramér-Rao [135]. Em nosso caso, isto significa que as nossas barras de erro serão as menores possíveis se assumimos um modelo gaussiano para calculá-las.

\subsection{Emaranhamento Hexapartite no OPO}

De posse da matriz espectral, podemos caracterizar as propriedades de emaranhamento do OPO considerando cada banda lateral como um modo do campo. Neste tipo de sistema, esperamos ter mais correlações do que quando olhamos somente para a parte real da matriz de covariâncias espectral, que representa a matriz de covariâncias dos operadores de soma e subtração de bandas laterais como discutido no capítulo 3 . $\mathrm{O}$ motivo para isto é a possibilidade de separar os modos mais correlacionados em partes diferentes de uma determinada bipartição.

Como mostrado na figura 7.6, as bandas laterais superior (inferior) do campo de bombeio devem estar correlacionadas com as bandas laterais superiores (inferiores) de cada feixe gêmeo assim como as banda lateral superior (inferior) de um dos gêmeos deve estar correlacionada com a banda lateral inferior (superior) do outro. Quando consideramos o sistema reduzido, descrito pela parte real da matriz de covariâncias 

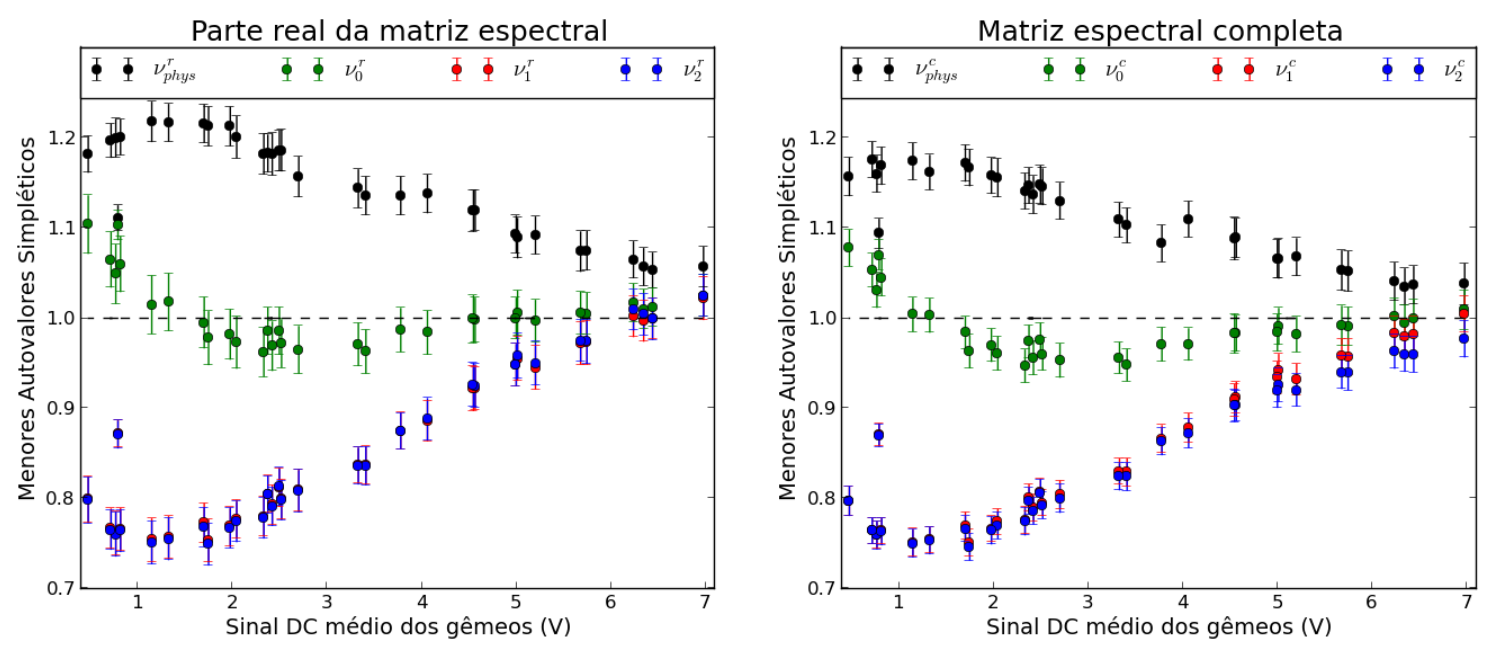

Figura 7.12: Comparação entre os menores autovalores simpléticos observados no sistema reduzido (esquerda) com os observados no sistema completo para as bipartições B0, B1 e B2 (direita). Note que há uma ligeira diminuição em todos os autovalores, principalmente nos gêmeos e em alta potência. Isto se deve ao fato de que, nestas medidas, há um aumento da parte imaginária das correlações de fase entre os feixes gêmeos, com mostrado na figura 7.11.

espectral, é necessário sempre colocar ambas as bandas laterais inferior e superior de cada campo em uma determinada parte. Esta é a única forma de garantir que os modos de soma (e subtração) de bandas laterais estarão sempre nesta mesma parte. No nosso sistema temos três bipartições desta forma:

- bipartição $B 0:\left(0_{+}, 0_{-}\right)$em uma parte e $\left(1_{+}, 1_{-}, 2_{+}, 2_{-}\right)$na outra

- bipartição $B 1:\left(1_{+}, 1_{-}\right)$em uma parte e $\left(0_{+}, 0_{-}, 2_{+}, 2_{-}\right)$na outra

- bipartição $B 2:\left(2_{+}, 2_{-}\right)$em uma parte e $\left(0_{+}, 0_{-}, 1_{+}, 1_{-}\right)$na outra

Consequentemente, não é possível separar os modos mais correlacionados, como, por exemplo, $\left(1_{+}, 2_{+}\right.$e $\left.0_{-}\right)$e $\left(1_{-}, 2_{-}\right.$e $\left.0_{+}\right)$formando as partes do sistema. Bipartições como esta de fato possuem uma violação maior do critério de emaranhamento do que as mostradas nas curvas da direita da figura 7.12 .

O sistema hexapartite possui 31 bipartições possíveis: 6 com um modo em uma das partes, 15 com dois modos em uma das partes e 10 com três modos em ambas as partes. As bipartições $B 0, B 1$ e $B 2$, mostradas em 7.12, são três das bipartições com dois campos em uma das partes. Na figura 7.13 são mostrados os menores autovalores simpléticos do sistema parcialmente transposto para todas as outras 28 bipartições. Podemos ver que há emaranhamento em todas elas e para quase toda a faixa de potências estudada. 

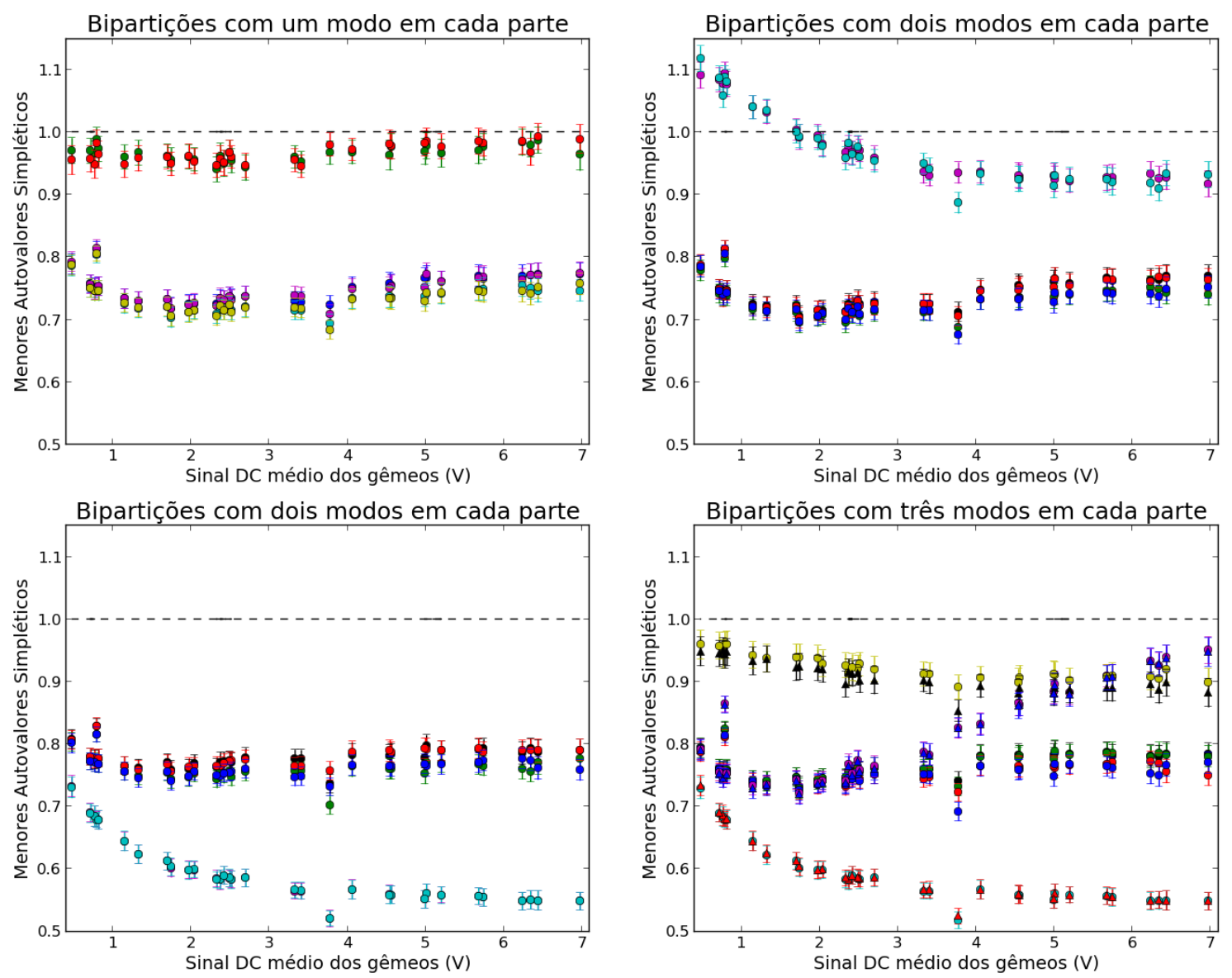

Figura 7.13: Autovalores simpléticos de todas as bipartições restantes. Na curva superior à esquerda, estão as bipartições com apenas um modo em uma das partes. É possível ver claramente, que cada banda lateral do campo de bombeio é fracamente emaranhada, mas o emaranhamento varia pouco com a frequência. Na curva superior à direita, são mostradas 6 das bipartições com dois modos em uma das partes. Estas são as bipartições para as quais esperamos menos emaranhamento, como $\left(1_{ \pm}, 2_{\mp}\right)$ e $\left(0_{+}, 0_{-}, 1_{\mp}, 2_{ \pm}\right)$. Na figura inferior à esquerda, são mostrados os menores autovalores simpléticos do sistema reduzido para as 6 bipartições restantes com dois modos em cada parte. Elas são as que esperamos possuir maior emaranhamento, como $\left(1_{ \pm}, 2_{ \pm}\right)$ $e\left(0_{+}, 0_{-}, 1_{\mp}, 2_{\mp}\right)$. Na figura inferior à direita, estão as 10 bipartições que possuem três modos em cada parte. Neste caso, há emaranhamento para todas a região de potências analisada.

\subsection{Comparação da Detecção Homodina com a Detecção com Cavidades}

Como discutido no capítulo 3, a detecção com cavidades possui uma vantagem em relação à detecção homodina espectral, pois aquela permite acessar desbalanceios de energia entre as bandas laterais enquanto esta é cega a possíveis desbalanceios porque trata as bandas laterais inferior e superior de maneira simétrica. Fizemos medidas utilizando uma cavidade de análise com as seguintes propriedades: $R_{\min }=0.05 \pm 0.01$, 
$\mathcal{F} \approx 260 \pm 10$ e $F S R=1.79 \pm 0.05 \mathrm{GHz}$, o que faz com que a sua largura de banda seja de $6.9 \pm 0.5 \mathrm{MHz}$.
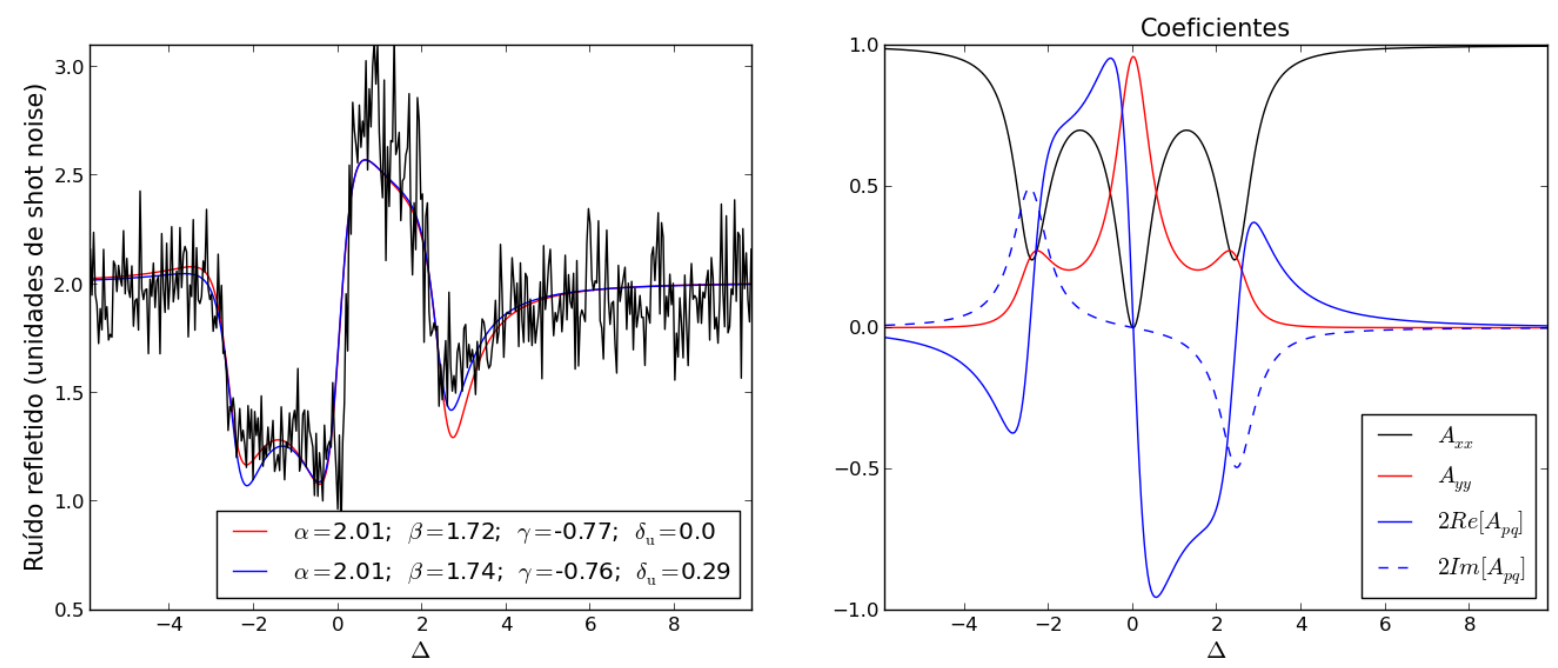

Figura 7.14: A figura à esquerda mostra a caracterização da matriz de covariâncias do campo gerado pelo OPO operando a uma potência 70\% acima do limiar de oscilação, $\sigma=1.7$. Os valores inferidos pelo ajuste estão mostrados na própria figura. A curva azul mostra o ajuste feito levando em consideração a presença de $\delta_{\mathrm{u}}$ e a curva vermelha mostra o ajuste feito forçando $\delta_{\mathrm{u}}$ a zero. A figura à direita mostra os coeficientes utilizados no ajuste.

O estado a ser caracterizado foi preparado no campo de bombeio refletido por um OPO operando acima do limiar. Utilizamos nestas medidas o OPO II, descrito no capítulo 4. Para que as correlações entre fase e intensidade sejam não-nulas, o OPO foi estabilizado a meia altura da ressonância do campo de bombeio. $\mathrm{O}$ foco desta investigação é o método de medida, por este motivo, não estamos especialmente interessados em observar efeitos quânticos no $O P O$, mas somente usá-lo como um estado prova para o sistema de detecção. Assim como no sistema com os três campos, o estado que nos interessa é aquele presente no campo logo antes da cavidade de análise. Um dos pontos importantes de se medir a parte imaginária das correlações espectrais é que teremos uma estimativa mais confiável da pureza do sistema, pois há informação acessível que não está sendo medida quando observamos somente a parte real das correlações. Esta informação perdida reflete como uma diminuição da pureza no estado observado. Considerando o estado que preparamos com gaussiano, a pureza global de um estado efetivo $\left(\delta_{\mathfrak{u}}=0\right)$ e com a medida completa nos permitindo estimar o estado total são

$$
\operatorname{Tr}\left[\left(\hat{\rho}_{e f f}\right)^{2}\right] \approx 0.35 \text { e } \operatorname{Tr}\left[\left(\hat{\rho}_{t o t}\right)^{2}\right] \approx 0.44
$$

Note que há um aumento considerável da pureza somente devido a uma melhor 
estimativa da matriz de covariâncias do estado.

Entretanto, como pode ser visto pelas medidas mostradas na figura 7.14, a parte imaginária neste sistema é muito pequena e não nos permite dizer que há de fato um desbalanceio entre as bandas laterais induzido pelo OPO. Esta se configura como uma medida preliminar e novas medidas estão sendo levadas a cabo no momento e para as quais preparamos um estado com um desbalanceio apreciável entre as bandas laterais. Para as novas medidas utilizamos uma cavidade com casamento de impedâncias para gerar o desbalanceio. Como não estamos interessados em propriedades de squeezing ou emaranhamento, este um meio de preparação de estados mais eficaz e mais simples de controlar que o OPO.

\subsection{Discussão sobre Gaussianidade}

Um ponto importante no nosso sistema, e utilizado extensivamente durante este trabalho, especialmente no estudo da robustez frente a perdas, diz respeito à gaussianidade da fotocorrente. Esta é associada à gaussianidade do estado das bandas laterais quando a fotocorrente pode ser descrita por um processo estacionário [66]. A forma que usarei aqui para buscar indicações é a partir de um teste de hipótese, precedimento muito utilizado em análise estatística clássica e aplicado em diversas áreas do conhecimento, especialmente em Medicina [136].

\subsubsection{Teste de Hipóteses}

A ideia de um teste de hipótese básico consiste em buscar indicações de que uma determinada suposição feita sobre alguma propriedade estatística é consistente com certo conjunto de dados. É importante se ter em mente que um teste deste tipo não permite refutar ou confirmar a hipótese, mas apenas dizer, com um determinado grau de confiabilidade, o quanto o conjunto de dados testado é discrepante do que seria esperado caso a hipótese fosse válida. O teste tem 3 elementos principais. O primeiro deles é a hipótese a ser testada, $H_{0}$, chamada de hipótese nula. O segundo é a chamada estatística de teste, $T$, que é uma função construída a partir dos dados experimentais cuja distribuição de probabilidades é bem conhecida quando a hipótese nula é verdadeira. Uma característica importante dessa estatística é que quanto mais discrepante a hipótese nula está do comportamento dos dados observados, maior deve ser o seu valor observado. Essas restrições ficarão mais claras no exemplo que darei mais à frente. 

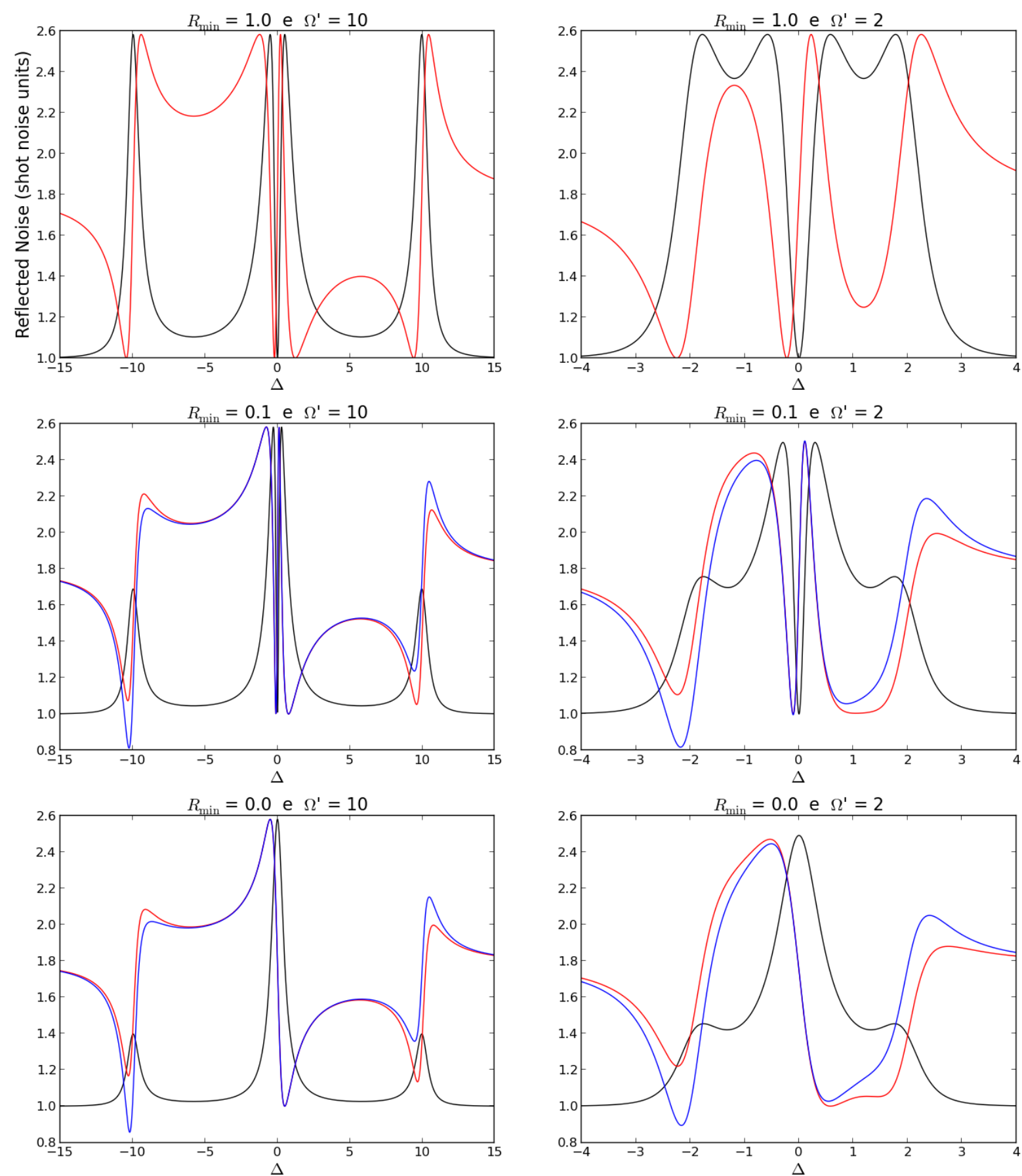

Figura 7.15: Estas figuras mostram o espectro de ruído de intensidade do campo refletido pela cavidade em diversas situações. Nas curvas à esquerda a ressonância com cada banda lateral e com a portadora estão bem resolvidas porque a frequência de análise é bem maior que a largura de banda. Nas da direita a ressonância com as bandas laterais se mistura à com a portadora. As entradas da matriz de covariâncias utilizada nas curvas pretas são $\alpha=1.0, \beta=2.58, \gamma=0.0$. Nas curvas vermelhas, a mesma matriz é utilizadas, mas com as quadraturas giradas de $45^{\circ}$ : $\alpha=1.79, \beta=1.79, \gamma=0.79$. Nas curvas em azul, um termo $\delta_{\mathfrak{u}}=0.76$ é adicionado. Neste exemplo, $\delta_{\mathrm{u}}$ é calculado para que o estado formado pelos dois modos seja puro. 
O terceiro elemento de um teste de hipóteses é o chamado p-valor. Formalmente, o p-valor é a probabilidade de que a estatística de teste possua um valor pelo menos tão extremo quanto o que foi observado em um determinado experimento,

$$
p=P\left(T>t \mid H_{0}\right)=1-P(T \leq t \mid H 0)=1-F_{0}(t),
$$

onde $T$ é a estatística de teste teórica e $t$ é o valor observado dessa estatística extraída a partir dos dados experimentais. A função $F_{0}(t)=P(T \leq t \mid H 0)$ é a distribuição de probabilidade cumulativa da estatística de teste até o valor observado $t$ quando $H_{0}$ é verdadeira ${ }^{3}$ e é uma função crescente definida no intervalo [0,1] [136]. Para variáveis aleatórias contínuas, $F_{0}(t)=P\left(T \leq t \mid H_{0}\right)=\int_{-\infty}^{t} d t^{\prime} \phi\left(t^{\prime}\right)$ onde $\phi(t)$ é a densidade de probabilidades.

Se a hipótese $H_{0}$ for verdadeira, os p-valores possuem densidade de probabilidade uniforme, pois eles são, por definição, uma distribuição cumulativa. Para provar isto, considere duas variáveis aleatórias contínuas $X$ e $Y$ que podem assumir valores reais $x$ e $y$, respectivamente, tal que

$$
Y=F_{X}(x)=\int_{-\infty}^{x} d x^{\prime} \phi_{X}\left(x^{\prime}\right)
$$

e $\phi_{X}(x)$ e $\phi_{Y}(y)$ sejam as densidades de probabilidades de $X$ e $Y$ respectivamente. Como $0 \leq y \leq 1$,

$$
1=\int_{0}^{1} d y^{\prime} \phi_{Y}\left(y^{\prime}\right)=\int_{-\infty}^{\infty} d x^{\prime} \phi_{X}\left(x^{\prime}\right)=\int_{0}^{1}|J|^{-1} d y^{\prime} \phi_{X}\left[F_{X}^{-1}\left(Y^{\prime}\right)\right]=\int_{0}^{1} d y^{\prime},
$$

pois $J=\partial y^{\prime} / \partial x^{\prime}=\phi_{X}\left(x^{\prime}\right)$. Portanto, $\phi_{Y}\left(y^{\prime}\right)=1$, ou seja, a distribuição de $Y$ é uniforme, pois esta está definida no intervalo $[0,1]$. Como o p-valor é igual a uma distribuição uniforme mais uma constante, ele é, por sua vez, também uma distribuição uniforme. Em outras palavras como, o p-valor é definido como uma distribuição cumulativa condicionado à hipótese $H_{0}$ ser válida, se observamos uma distribuição uniforme dos p-valores temos evidências para aceitar a hipótese $H_{0}$ como sendo válida. $\mathrm{Ou}$, de forma menos restritiva: não temos nenhuma evidência que permita refutar a validade da hipótese $H_{0}$.

Normalmente é escolhido um valor $\alpha$, chamado de significância estatística, abaixo do qual a hipótese deve ser considerada falsa 4 A escolha deste valor é arbitrária e quanto mais próxima de zero, mais o teste de hipótese é capaz de rejeitar a hipótese nula. O problema é que mesmo quando a hipótese é verdadeira, uma fração dos p-

\footnotetext{
${ }^{3}$ Ela é igual à probabilidade da variável aleatória assumir valores $t \leq t^{\prime}$ assumindo que $H_{0}$ esteja correta.

${ }^{4}$ Valores bem comuns são $\alpha=0.01,0.05$.
} 
valores vai estar abaixo do nível de significância escolhido, pois a distribuição dos mesmos deve ser uniforme. Por este motivo, uma informação bem mais confiável pare rejeitar a hipótese inicial é olhar para a distribuição dos p-valores em vez de somente para um p-valor individual. Se vários p-valores possuírem, consistentemente, valores abaixo do nível de significância, então a hipótese pode ser rejeitada satisfatoriamente.

\section{Comparação entre as distribuições Gaussiana e T de Student}
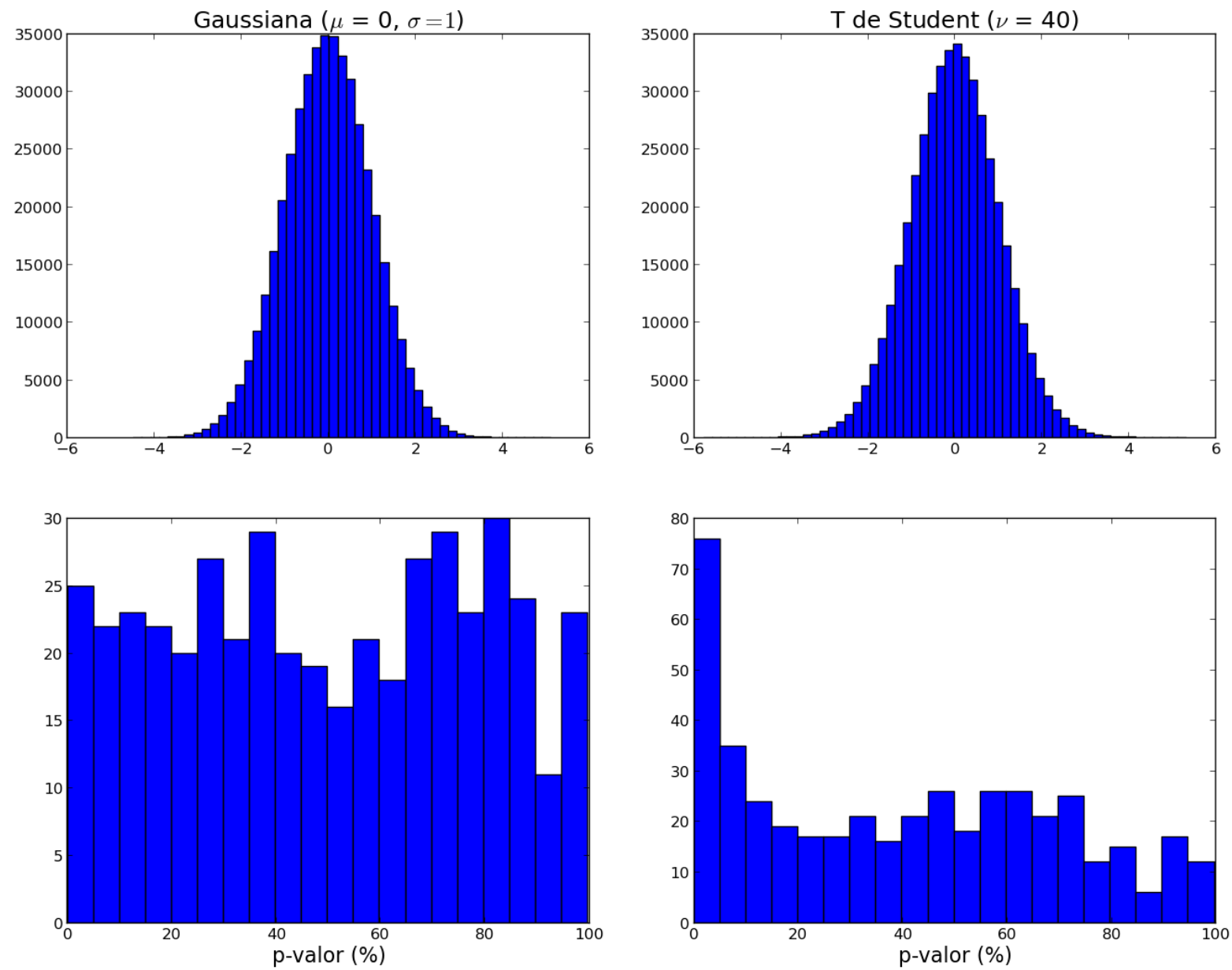

Figura 7.16: Comparação do resultado do teste de Shapiro-Wilk para uma distribuição gaussiana com uma $T$ de Student de dimensão $v=40$, ambas produzidas por um gerador de números pseudo-aleatórios. O ponto interessante desta comparação é que, além do apelo visual, o quarto momento da distribuição T de Student é apenas $8 \%$ maior que o da distribuição gaussiana. Note que o resultado para o teste de Shapiro-Wilk acusa claramente a distribuição da direita não é gaussiana.

Discutirei um exemplo simples de teste de hipóteses para criar um pouco de intuição sobre a estatística de teste e sobre o p-valor. Considere que possuo um conjunto de variáveis aleatórias, $x_{1}, x_{2}, \ldots, x_{n}$, com distribuição gaussiana de média $\mu$ e variância $\sigma$, e quero testar a hipótese de que $\mu=0$. De posse de uma hipótese nula, agora precisamos 
de uma estatística de teste. Uma possibilidade simples seria

$$
T_{1} \equiv \bar{x}=\frac{1}{n} \sum_{i=1}^{n} x_{i}
$$

Como cada $x_{i}$ segue uma distribuição gaussiana, de média $\mu$ e variância $\sigma^{2}$, a distribuição de $T_{1}$ é uma gaussiana de média $\mu$ e variância $\sigma^{2} / n$. Entretanto, ela é possui um problema, pois a distribuição de probabilidade de $T_{1}$ é simétrica em relação $\mu$, ou seja, ela decresce á medida que uma realização de $T_{1}$ se afasta de $\mu$ em ambos os sentidos positivo e negativo. De fato, o ponto que satisfaz a hipótese nula está na própria mediana da distribuição. Para contornar este problema, um estatística de teste melhor que $T_{1}$ é $T_{2}=T_{1}^{2}$, que corresponde a projetar todos os valores negativos sobre os positivos. Neste caso, a probabilidade de que cada realização de $T_{2}$ se afasta de $\mu$ decresce a medida que o valor seu valor aumenta. Como a probabilidade de uma ocorrência de um valor em $T_{2}$ deve ser a igual à soma de toda possível ocorrência $T_{1}$ mais o seu respectivo valor negativo, $P\left(T_{2}\right) d T_{2}=P\left(T_{1}\right) d T_{1}+P\left(-T_{1}\right) d T_{1}$, então, $T_{2}$ segue a densidade de probabilidades

$$
P\left(T_{2}\right)=\frac{1}{\sqrt{2 \pi \sigma^{2} T_{2}}} e^{-\frac{T_{2}}{2 \sigma^{2}}}
$$

caso $\sigma$ seja conhecidd 5 Portanto, é possível comparar $T_{2}$ calculado a partir da distribuição $P\left(T_{2}\right)$ com com o valor $t_{2}=\left[\sum x_{i}^{d}\right]^{2} / n^{2}$, calculado a partir dos dados que temos disponíveis.

\subsubsection{Teste de Shapiro-Wilk}

O teste de Shapiro-Wilk é um teste de hipóteses no qual a hipótese nula, $H_{0}$, é de que a distribuição que gerou os dados é gaussiana. Como dito antes, este teste não permite responder se o estado é gaussiano ou não, mas somente nos fornece um método quantitativo de testar o quão discrepante são os dados observados do que seria esperado se os mesmos fossem gerados por uma distribuição gaussiana. A estatística de teste deste método é [137]

$$
T=\frac{\left(\sum_{i=1}^{n} a_{i} x_{(i)}\right)^{2}}{\sum_{i=1}^{n}\left(x_{i}-\bar{x}\right)^{2}}, \quad \text { onde } \quad \bar{x}=\frac{1}{n} \sum_{i=1}^{n} x_{i} \quad \text { e } \quad a=\frac{\left(V^{-1} m\right)^{T}}{\sqrt{\left(V^{-1} m\right)^{T} V^{-1} m}} .
$$

O valor $x_{(i)}$ é o $i$-ésimo menor valor na amostra e é chamado de ordem da amostra. Ele é a componente $i$ do vetor formado pela amostra ordenado de maneira crescente. As quan-

\footnotetext{
${ }^{5}$ Esta distribuição é um caso particular da distribuição $\chi^{2}$.
} 
tidades $m_{i}=\left\langle x_{(i)}\right\rangle$ e $V_{i j}=\left\langle\left(x_{(i)}-m_{i}\right)\left(x_{(j)}-m_{j}\right)\right\rangle$ são os primeiros e segundos momentos das ordens da amostra. Para se ter uma ideia do poder do teste, fiz uma comparação

HF da medida 16.dat; campo 1
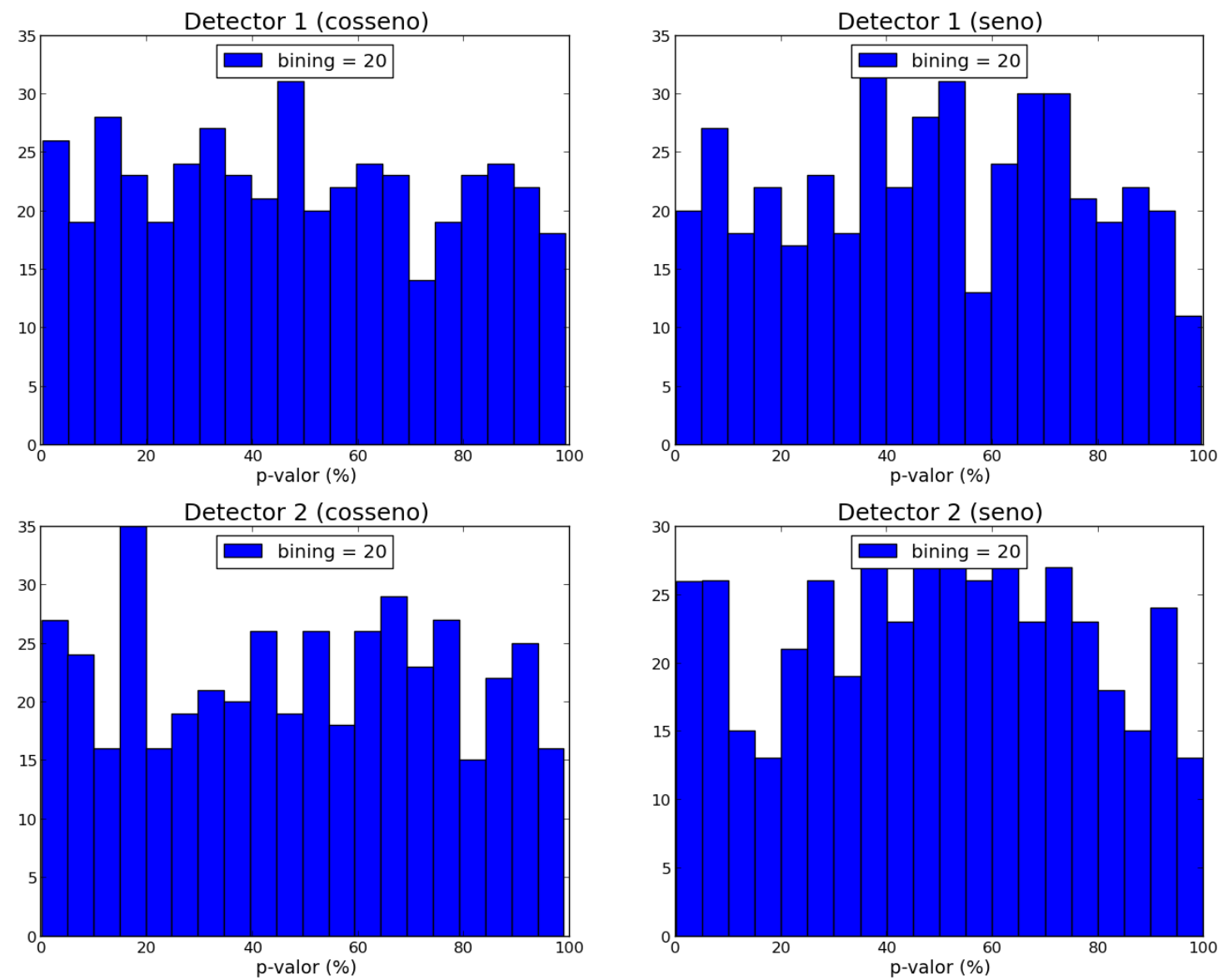

Figura 7.17: Teste de Shapiro-Wilk para um dos campos para a mesma aquisição mostrada em 7.11. O teste é feito para o dado bruto, sem nenhuma correção ou tratamento posterior. Note que pela uniformidade dos p-valores, temos uma forte indicação de que o nosso estado é, de fato, gaussiano.

de uma distribuição gaussiana, $g(x)$, como uma T de Student, $t_{v}(x)$, definidas por

$$
g(x)=\frac{1}{\sqrt{2 \pi}} \mathrm{e}^{-\frac{1}{2} x^{2}} \text { e } t_{v}(x)=\frac{\Gamma\left(\frac{v+1}{2}\right)}{\sqrt{v \pi} \Gamma\left(\frac{v}{2}\right)}\left(1+\frac{x^{2}}{v}\right)^{-\frac{v+1}{2}},
$$

onde $\Gamma(x)=\int_{0}^{\infty} t^{x-1} e^{-t} d t$ é a função Gamma. A distribuição T de student tem dois casos particulares bem conhecidos. Quando $v=1$, ela se torna uma função lorenztiana e quando $v \rightarrow \infty$, ela tende a um gaussiana. Ambas as distribuições mostradas em (7.18) possuem média nula variância $\sigma^{2}=11^{6}$. Como ambas são simétricas em relação a $x$, todos

\footnotetext{
${ }^{6}$ A média da distribuição T de Student só está definida para $v>1$ e a variância para $v>2$.
} 
os momentos ímpares também se anulam. A comparação do teste de gaussianidade para elas é mostrada na figura 7.16 e para tal escolhi $t_{40}(x)$. Como os quartos momentos (kurtosis) para as distribuições T de Student e gaussiana são dados por

$$
\mu_{g}=\int_{-\infty}^{\infty} d x^{4} g(x)=3 \quad \text { e } \quad \mu_{T}=\int_{-\infty}^{\infty} d x^{4} t_{v}(x)=3\left[1+\frac{2}{v-4}\right]
$$

tal que $\mu_{T}$ só está definido para $v>4, \mu_{T}=1,08 \mu_{g}$ para $v=40$.

Como pode ser visto na figura 7.17, os estados que produzimos com o OPO são consistentes com uma estatística gaussiana para as quadraturas. Note que durante esta medida, a cavidade de análise está sendo varrida. Isto faz com que os dados apresentados correspondam à medida de mais de uma quadratura do campo. Como não houve discrepâncias com o que era esperado de uma distribuição gaussiana para as diversas distribuições marginais, é seguro afirmar que a função de Wigner total é consitente com uma distribuição gaussiana. 


\section{Conclusões e Perspectivas}

Nesta tese, diversos assuntos foram abordados, a grande maioria relacionados á investigação do emaranhamento em variáveis contínuas. Parte do trabalho experimental foi realizado em conjunto com Antonio Sales Coelho e está descrito com mais detalhes em sua tese, em especial a produção do emaranhamento tripartite entre os três campos produzidos pelo OPO e a análise da robustez do emaranhamento neste caso.

As partes principais que abordei se referem à análise teórica e experimental da robustez do emaranhamento nos feixes gêmeos. Mostramos que o excesso na soma dos ruídos das quadratura, em relação ao ruído do vácuo, é o responsável pelo aparecimento de estados de emaranhamento frágil. Observamos que para determinadas regiões de potência, o emaranhamento entre os feixes gêmeos desaparece por completo para perdas parciais em um dos feixes gêmeos. Também demonstramos, a partir do critério $P P T$, uma condição suficiente para que um determinado estado emaranhado seja robusto. A qual também se torna necessária se o estado for gaussiano.

O outro ponto importantes tratado nesta tese se refere à análise da fotodetecção de feixes intensos do domínio da frequência. A abordagem que utilizada, tratando cada banda lateral separadamente, permite uma descrição mais rica e completa do estado produzido pelo OPO. Dentro desta descrição, mostramos que o OPO operando acima do limiar pode produzir estados de seis modos completamente inseparáveis e em que condições é lícito usar a aproximação de um modo por campo. Também mostramos que a detecção com cavidades é capaz de fazer uma medida completa do estado das bandas laterais quando este é gaussiano. Isto a coloca em uma posição superior à detecção homodina espectral, que é insensível a uma das entradas da matriz de covariâncias do sistema de dois modos.

Há perspectivas para a continuação deste trabalho em diversas linhas de pesquisa. As mais factíveis em curto prazo relacionadas a comunicação quântica e que já estão em andamento no laboratório são: a implementação de teletransporte e entanglement 
swapping entre diferentes frequências do espectro eletromagnético e a implementação de protocolos de memória quântica usando átomos de Rubídio. Para o teletransporte, já foram iniciadas a construção de um outro OPO com maior squeezing que o atual e das cavidades de análise que serão utilizadas para caracterizar o estado de entrada e de saída do protocolo de teletransporte. Para o procedimento o armazenamento, já há um $O P O$ em funcionamento no laboratório que opera nos comprimentos de onda de 780 nm (bombeio - linha atômica D2 do Rubídio) e 1560 (feixes gêmeos). O emaranhamento tripartite neste sistema pode ser usado para converter o estado presente nos átomos para o comprimento de onda de telecom e facilitar a comunicação em longas distâncias usando fibras óticas. Do ponto de vista do estudo da estrutura dos emaranhados que preparamos, há a possibilidade de que possamos preparar estados GHZ em variáveis contínuas, mais detalhes sobre isto podem ser encontrados na tese de Antonio Sales Coelho. Também há o interesse em entender melhor a estrutura do emaranhamento hexapartite produzido pelo OPO, como a possibilidade de preparar estados do tipo Cluster em variáveis contínuas [138] utilizando o OPO nesta configuração. 


\section{Referências Bibliográficas}

[1] M. A. Nielsen and I. L. Chuang. Quantum Computation and Quantum Information. Cambridge Univ. Press, 2000.

[2] C. H. Bennett, G. Brassard, C. Crépeau, R. Jozsa, A. Peres, and W. K. Wootters. "teleporting an unknown quantum state via dual classical and einstein-podolskyrosen channels". Phys. Rev. Lett., 70:1895-1899, Mar 1993.

[3] P. van Loock, S. L. Braunstein, and H. J. Kimble. Phys. Rev. A.

[4] C. H. Bennett and S. J. Wiesner. "communication via one- and two-particle operators on einstein-podolsky-rosen states". Phys. Rev. Lett., 69:2881-2884, Nov 1992.

[5] R. Jozsa and N. Linden. "on the role of entanglement in quantum-computational speed-up". Proc. R. Soc. Lond. A, 459:2011-2032, Aug 2003.

[6] E. Knill. "quantum computing". Nature, 463:441-443, Jan 2010.

[7] A. Stern and N. H. Lindner. "topological quantum computation-from basic concepts to first experiments". Science, 339(6124):1179-1184, 2013.

[8] J. H. Kimble. "the quantum internet". Nature, 453:1023-1030, Jun 2008.

[9] A. I. Lvovsky, B. C. Sanders, and W. Tittel. "optical quantum memory". Nat. Phot., 3(706-714), 2009.

[10] P. K. Lam and T. C. Ralph. "quantum cryptography: Continuous improvement". Nat. Phot., 7(350-352), Apr 2013.

[11] M. Martinelli, C. L. Garrido Alzar, P. H. Souto Ribeiro, and P. Nussenzveig. "classical and quantum properties of optical parametric oscillators". "Brazilian Journal of Physics

, 31:597-615, 122001.

[12] M. Martinelli, J. A. O. Huguenin, P. Nussenzveig, and A. Z. Khoury. “orbital angular momentum exchange in an optical parametric oscillator". Phys. Rev. A, 70:013812, Jul 2004.

[13] A. S. Villar, L .S. Cruz, K. N. Cassemiro, M. Martinelli, and P. Nussenzveig. Generation of bright two-color continuous variable entanglement. Phys. Rev. Lett., 95:243603, Dec 2005.

[14] A. S. Villar, C. Fabre, M. Martinelli, and P. Nussenzveig. "direct production of tripartite pump-signal-idler entanglement in the above-threshold optical parametric oscillator". Phys. Rev. Lett., 97:140504, Oct 2006. 
[15] A. S. Villar, K. N. Cassemiro, K. Dechoum, A. Z. Khoury, M. Martinelli, and P. Nussenzveig. "entanglement in the above-threshold optical parametric oscillator". J. Opt. Soc. Am. B, 24(2):249-256, Feb 2007.

[16] K. N. Cassemiro, A. S. Villar, P. Valente, M. Martinelli, and P. Nussenzveig. "experimental observation of three-color optical quantum correlations". Opt. Lett., 32(6):695-697, Mar 2007.

[17] A. S. César, J. E. S. and, K. N. Cassemiro, A. S. Villar, M. Lassen, P. Nussenzveig, and M. Martinelli. "extra phase noise from thermal fluctuations in nonlinear optical crystals". Phys. Rev. A, 79(063816), Jun 2009.

[18] C. H. Bennett, H. J. Bernstein, S. Popescu, and B. Schumacher. "concentrating partial entanglement by local operations". Phys.Rev.A, 53:2046, 1996.

[19] H. J. Briegel, W. Dür, J. I. Cirac, and P. Zoller. "quantum repeaters: The role of imperfect local operations in quantum communication". Physical Review Letters, 81(26):5932-5935, December 1998.

[20] J-W. Pan, S. Gasparoni, R. Ursin, G. Weihs, and A. Zeilinger. "experimental entanglement purification of arbitrary unknown states". Nature, 423:417-422, Mar 2003.

[21] B. Hage, A. Samblowski, J. DiGuglielmo, A. Franzen, J. Fiurášek, and R. Schnabel. "preparation of distilled and purified continuous-variable entangled states". Nat. Phys., 4(915-918), Dec 2008.

[22] R. Dong, M. Lassen, J. Heersink, C. Marquardt, R. Filip, G. Leuchs, and U. L. Andersen. "experimental entanglement distillation of mesoscopic quantum states". Nat. Phys., 4(919-922), Nov 2008.

[23] N. Sangouard, C. Simon, H. de Riedmatten, and N. Gisin. "quantum repeaters based on atomic ensembles and linear optics". Rev. Mod. Phys., 83:33-80, Mar 2011.

[24] A. I. Lvovsky and M. G. Raymer. "continuous-variable optical quantum-state tomography". Rev. Mod. Phys., 81:299-332, Mar 2009.

[25] K. Vogel and H. Risken. "determination of quasiprobability distributions in terms of probability distributions for the rotated quadrature phase". Phys. Rev. A, 40:2847-2849, Sep 1989.

[26] A. S. Coelho, F. A. S. Barbosa, K. N. Cassemiro, A. S. Villar, M. Martinelli, and P. Nussenzveig. "three-color entanglement". Science, 326(5954):823-826, 2009.

[27] F. A. S. Barbosa, A. S. Coelho, A. J. Faria, K. N. Cassemiro, A. S. Villar, P. Nussenzveig, and M. Martinelli. "robustness of bipartite gaussian entangled beams propagating in lossy channels". Nat. Phot., 4(858), Mar 2010.

[28] B. Hage, A. Samblowski, and R. Schnabel. "towards einstein-podolsky-rosen quantum channel multiplexing". Phys. Rev. A, 81:062301, Jun 2010. 
[29] A. Einstein, B. Podolski, and N. Rosen. "can quantum-mechanical description of physical reality be considered complete?". Phys. Rev., 47:777-780, 1935.

[30] N. Bohr. "can quantum-mechanical description of physical reality be considered complete?". Phys. Rev., 48:696, 1935.

[31] E. Schrödinger. "discussion of probability relations between separated systems". Proc. Cambr. Phil. Soc., 31:555, 1935.

[32] E. Schrödinger. "probability relations between separated systems". Proc. Cambr. Phil. Soc., 32:446, 1936.

[33] D. Bohm. Quantum Theory. New York: Prentice Hall, 1951.

[34] J.S. Bell. "on the problem of hidden variables in quantum mechanics". Rev. Mod. Phys., 38:447-452, Jul 1966.

[35] D. Bohm. "a suggested interpretation of the quantum theory in terms of "hidden"variables i.". Phys. Rev., 85:166-179, Jan 1952.

[36] D. Bohm. "a suggested interpretation of the quantum theory in terms of "hidden"variables ii.". Phys. Rev., 85:180-193, Jan 1952.

[37] J. S. Bell. “on the einstein podolsky rosen paradox". Physics, 1:195-200, 1964.

[38] J. F. Clauser and A. Shimony. "proposed experiment to test local hidden-variable theories". Rep. Prog. Phys, 41:1881-1928, 1978.

[39] S. J. Freedman and J. F. Clauser. "experimental test of local hidden-variable theories". Phys. Rev. Lett., 28:938-941, Apr 1972.

[40] A. Aspect. "coherent population trapping in laser spectroscopy". Phys. Rev. Lett., 35:257-354, 1981.

[41] A. Aspect, J. Dalibard, and G. Roger. "experimental test of bell's inequalities using time- varying analyzers". Phys. Rev. Lett., 49:1804-1807, Dec 1982.

[42] R. F. Werner. "quantum states with einstein-podolsky-rosen correlations admitting a hidden-variable model". Phys. Rev. A, 40:4277-4281, 1989.

[43] J. D. Jackson. Probability Theory: The Logic of Science. Cambridge University Press, 2003.

[44] V. Vedral. Introduction to Quantum Information Science. Oxford University Press, 2006.

[45] C. Cohen-Tannoudji, B. Diu, and F. Laloe. Quantum Mechanics. Wiley-VCH,Vol1-2, 2005.

[46] J. J. Sakurai. Modern Quantum Mechanics. Addison-Wesley, 1994.

[47] Messiah. Quantum Mechanics. Dover, 1999. 
[48] T. Scheidl, R. Ursin, J. Kofler, S. Ramelow, X.-S. Ma, T. Herbst, L. Ratschbacher, A. Fedrizzi, N. K. Langford, T. Jennewein, and A. Zeilinger. "violation of local realism with freedom of choice". Proceedings of the National Academy of Sciences, 107(46):19708-19713, 2010.

[49] E. B. Rosa and N. E. Dorsey. "the ratio of the electromagnetic and electrostatic units". Phys. Rev. (Series I), 22:367-368, Jun 1906.

[50] J. S. Bell. Speakable and Unspeakable in Quantum Mechanics. Cambridge University Press, 2004.

[51] Nicolas Gisin. "bell inequalities: Many questions, a few answers". In Quantum Reality, Relativistic Causality, and Closing the Epistemic Circle, volume 73 of The Western Ontario Series in Philosophy of Science, pages 125-138. Springer Netherlands, 2009.

[52] S. Goldstein, T. Norsen, D. V. Tausk, and N. Zanghi. "bell's theorem". Scholarpedia, 6:8378, 2011.

[53] N. Brunner, D. Cavalcanti, S. Pironio, V. Scarani, and S. Wehner. "bell nonlocality".

[54] L.-A. Wu, M. Xiao, and H. J. Kimble. "squeezed states of light from an optical parametric oscillator". J. Opt. Soc. Am. B, 4(10):1465-1475, Oct 1987.

[55] R. W. Boyd. Nolinear Optics. Academic Press, 2003.

[56] A. Yariv. Quantum Electronics. Wiley, 1988.

[57] D. C. Burnham and D. L. Weinberg. "observation of simultaneity in parametric production of optical photon pairs". Phys. Rev. Lett., 25:84-87, Jul 1970.

[58] C. Monroe. "quantum information processing with atoms and photons". Nature, 416:238-246, Mar 2002.

[59] H. Kogelnik and T. Li. "laser beams and resonators". Appl. Opt., 5(10):1550-1567, Oct 1966.

[60] J. D. Jackson. Classical Electrodynamics. Wiley, 1962.

[61] D. J. Griffiths. Introduction to Electrodynamics. Prentice Hall, 1999.

[62] L.Mandel and E. Wolf. Optical Coherence and Quantum Optics. Cambridge University Press, 1995.

[63] T. Debuisschert, A. Sizmann, E. Giacobino, and C. Fabre. “type-ii continuouswave optical parametric oscillators: oscillation and frequency-tuning characteristics". J. Opt. Soc. Am. B, 10:1668-1680, Sep 1993.

[64] R. Graham and H. Haken. "the quantum-fluctuations of the optical parametric oscillator i". Zeitschrift fur Physik, 210:276-302, June 1968.

[65] R. Graham. "the quantum-fluctuations of the optical parametric oscillator ii". Zeitschrift fur Physik, 210:319, June 1968. 
[66] F. A. S. Barbosa, A. S. Coelho, K. N. Cassemiro, P. Nussenzveig, M. Martinelli, C. Fabre, and A. S. Villar. "". a ser submetido",.

[67] F. A. S. Barbosa. "relatório científico fapesp".

[68] M. Martinelli. "comunicação interna".

[69] A. S. Villar. "comunicação interna".

[70] C. Cohen-Tannoudji; J. Dupont-Roc; G. Grynberg. Photons and Atoms-Introduction to Quantum Electrodynamics. Wiley, 1992.

[71] Roy J. Glauber. "the quantum theory of optical coherence". Phys. Rev., 130(6):2529-2539, Jun 1963.

[72] C. Cohen-Tannoudji; J. Dupont-Roc; G. Grynberg. Atom-Photon Interactions-Basic Processes and Applications. Wiley, 1992.

[73] M. O. Scully and M. S. Zubairy. Quantum Optics. Cambridge University Press, 1997.

[74] Roy J. Glauber. "coherent and incoherent states of the radiation field". Phys. Rev., 131(6):2766-2788, Sep 1963.

[75] H. Goldstein. Classical Mechanics. Addison Wesley, 2001.

[76] L. Landau and E. M. Lifshitz. Mechanics. Butterworth-Heineman, 3ed, 1976.

[77] H. P. Yuen and J. H. Shapiro. "optical communication with two-photon coherent states-part i: Quantum-state propagation and quantum-noise". Information Theory, IEEE Transactions on, 24(6):657-668, 1978.

[78] J. H. Shapiro, H. P. Yuen, and A. Mata. "optical communication with twophoton coherent states-part ii: Photoemissive detection and structured receiver performance". Information Theory, IEEE Transactions on, 25(2):179-192, 1979.

[79] H. P. Yuen and J. H. Shapiro. "optical communication with two-photon coherent states-part iii: Quantum measurements realizable with photoemissive detectors". Information Theory, IEEE Transactions on, 26(1):78-92, 1980.

[80] P. Galatola, L.A. Lugiato, M.G. Porreca, P. Tombesi, and G. Leuchs. "system control by variation of the squeezing phase. Optics Communications, 85(1):95 $103,1991$.

[81] A. S. Villar. "the conversion of phase to amplitude fluctuations of a light beam by an optical cavity". Am. J. Phys., 2008.

[82] E. H. Huntington, G. N. Milford, C. Robilliard, T. C. Ralph, O. Glöckl, U. L. Andersen, S. Lorenz, and G. Leuchs. Demonstration of the spatial separation of the entangled quantum sidebands of an optical field. Phys. Rev. A, 71:041802, Apr 2005.

[83] H. P. Yuen and V. W. S. Chan. "noise in homodyne and heterodyne detection". Opt. Lett., 8(3):177-179, Mar 1983. 
[84] B. L. Schumaker. "noise in homodyne detection". Opt. Lett., 9(5):189-191, May 1984.

[85] A. I. Lvovsky. "iterative maximum-likelihood reconstruction in quantum homodyne tomography". Journal of Optics B: Quantum and Semiclassical Optics, 6(6):S556, 2004.

[86] V. B. Braginsky, F. Y. Khalili, and K. S. Thorne. Quantum Measurement. Cambridge University Press, 1995.

[87] C. W. Gardiner. Quantum Noise. Springer-Verlag, 1991.

[88] M. B Priestley. Spectral Analysis and Time Series. Academic Press, 1981.

[89] L.-M Duan, G. Giedke, J. I. Cirac, and P. Zoller. “inseparability criterion for continuous variable systems". Phys. Rev. Lett., 84:2722, 2000.

[90] M. Martinelli. Compressção de Ruído Quântico e Efeitos Transeversos em Osciladores Paramétricos óticos. PhD thesis, Universidade de São Paulo, 2002.

[91] A. S. Villar. Emaranhamento Multicolor entre Feixes Intensos de Luz. PhD thesis, Universidade de São Paulo, 2007.

[92] K. N. Cassemiro. Correlações Quânticas Multicolores no Oscilador Paramétrico Ótico. PhD thesis, Universidade de São Paulo, 2008.

[93] G. D. Boyd and D. A. Kleinman. "parametric interaction of focused gaussian light beams". J. Appl. Phys., 39(8):3597-3639, 1968.

[94] P. Horowitz and W. Hill. The Art of Electronics. Cambridge University Press, 1989.

[95] E. D. Black. "an introduction to pound-drever-hall laser frequency stabilization". American Journal of Physics, 69(1):79-87, 2001.

[96] F. A. S. Barbosa, A. J. de Faria, A. S. Coelho, K. N. Cassemiro, A. S. Villar, P. Nussenzveig, and M. Martinelli. "disentanglement in bipartite continuousvariable systems". Phys. Rev. A, 84:052330, Nov 2011.

[97] R. Horodecki, P. Horodecki, M. Horodecki, and K. Horodecki. "quantum entanglement". Rev. Mod. Phys., 81(865), Jun 2009.

[98] K. Ball. An Elementary Introduction to Modern Convex Geometry - Flavors of Geometry vol. 31". MSRI Publications, 1997.

[99] M. Horodecki, P. Horodecki, and R. Horodecki. "separability of mixed states: Necessary and sufficient conditions". Phys. Lett. A, 223:1-8, 1996.

[100] V. Vedral. "the role of relative entropy in quantum information theory". Rev. Mod. Phys., 74(197), Mar 2002.

[101] A. Peres. "separability criterion for density matrices". Phys. Rev. Lett., 77:1413-1416, 1996. 
[102] R. F. Werner and M. M. Wolf. "bound entangled gaussian states". Phys. Rev. Lett., 86(3658), Abr 2001.

[103] V. Giovannetti, S. Mancini, D. Vitali, and P. Tombesi. "inseparability criterion for continuous variable systems". Phys. Rev. A, 67:022320, 2003.

[104] W. Vogel E. Shchukin. "inseparability criteria for continuous bipartite quantum states". Phys. Rev. Lett., 95(230502), Nov 2005.

[105] "entropic entanglement criteria for continuous variables".

[106] A. Bohm and K. Kraus. States Effects and Operations: Fundamental Notions of Quantum Theory (Lecture Notes in Physics). Spring-Verlag, 1983.

[107] R. Simon. "peres-horodecki separability criterion for continuous variable systems". Phys. Rev. Lett., 84:2726, 2000.

[108] W. Schleich. Quantum Optics in Phase Space. Wiley-VCH, 2001.

[109] J. E. Moyal. Quantum mechanics as a statistical theory. Mathematical Proceedings of the Cambridge Philosophical Society, 45:99-124, Jan 1949.

[110] W. Vogel and D.-G. Welsch. Quantum Optics. Wiley, 2001.

[111] E. Wigner. "on the quantum correction for thermodynamic equilibrium". Phys. Rev., 40(749), Jun 1932.

[112] R.L. Hudson. "when is the wigner quasi-probability density non-negative?". Reports on Mathematical Physics, 6(2):249 - 252, 1974.

[113] S. L. Braustein and P. van Loock. "quantum information with continuous variables". Rev. Mod. Phys., 77:513-577, 2005.

[114] C. Weedbrook, S. Pirandola, R. García-Patrón, N. J. Cerf, T. C. Ralph, J. H. Shapiro, and S. Lloyd. "gaussian quantum information". Rev. Mod. Phys., 84:621-669, May 2012.

[115] M. G. A. Paris, F. Illuminati, A. Serafini, and S. Siena. "purity of gaussian states: Measurement schemes and time evolution in noisy channel". 68, 2003.

[116] H. P. Robertson. "the uncertainty principle". Phys. Rev., 34(163), 1929.

[117] V. Arnold. Mathematical Methods of Classical Mechanics. Springer, 1989.

[118] R. Simon, N. Mukunda, and B. Dutta. "quantum-noise matrix for multimode systems: U(n) invariance, squeezing, and normal forms". Phys. Rev. A, 49:1567, 1994.

[119] J. Williamson. "on the algebraic problem concerning the normal forms of linear dynamical systems". American Journal of Mathematics, 58:141-163, Jan 1936.

[120] K. Huang. Statistical Mechanics. Wiley, 1987.

[121] S. R. A. Salinas. Mecânica Estatística. Edusp, 2005. 
[122] R. Simon, E. C. G. Sudarshan, and N. Mukunda. "gaussian-wigner distributions in quantum mechanics and optics". Phys. Rev. A, 36:3868-3880, 1987.

[123] R. Simon., E. C. G. Sudarshan, and N. Mukunda. "gaussian pure states in quantum mechanics and the symplectic group". Phys. Rev. A, 37:3028-3038, 1988.

[124] W. P. Bowen, R. Schnabel, P. K. Lam, and T. C. Ralph. "experimental investigation of criteria for continuous variable entanglement". Phys. Rev. Lett., 90:043601, Jan 2003.

[125] M. Horodecki and P. Horodecki. "reduction criterion of separability and limits for a class of distillation protocols". Phys. Rev. A, 59:4206-4216, Jun 1999.

[126] "open problems in quantum information theory". http://qig.itp.unihannover.de/qiproblems/2.

[127] J. Sperling and W. Vogel. "non-existence of bipartite bound entanglement with negative partial transpositions".

[128] D. F. Walls and G. J. Milburn. Quantum Optics. Spring-Verlag, 1994.

[129] D. Wang, Y. Shang, Z. Yan, W. Wang, X. Jia, C. Xie, and K. Peng. “experimental investigation about the influence of pump phase noise on phase-correlation of output optical fields from a non-degenerate parametric oscillator". EPL (Europhysics Letters), 82(2):24003, 2008.

[130] T. Yu and J. H. Eberly. "sudden death of entanglement". Science, 323(5914):598601, 2009.

[131] M. P. Almeida, F. de Melo, M. Hor-Meyll, A. Salles, S. P. Walborn, P. H. Souto Ribeiro, and L. Davidovich. "environment-induced sudden death of entanglement". Science, 316(5824):579-582, 2007.

[132] J. Eisert, S. Scheel, and M. B. Plenio. "distilling gaussian states with gaussian operations is impossible". Phys. Rev. Lett., 89:137903, Sep 2002.

[133] G. Giedke and J. I. Cirac. "characterization of gaussian operations and distillation of gaussian states". Phys. Rev. A, 66:032316, Sep 2002.

[134] J. Fiurášek. "gaussian transformations and distillation of entangled gaussian states". Phys. Rev. Lett., 89:137904, Sep 2002.

[135] E.L. Lehmann and G. Casella. Theory of Point Estimation. Springer, 2ed, 1988.

[136] E.L. Lehmann and J. P. Romano. Testing Statistical Hypotheses. Springer, 6ed, 2005.

[137] S. S. Shapiro and M. B. Wilk. "an analysis of variance test for normality (complete samples)". Biometrika, 52(3 and 4):591 - 611, 1965.

[138] J. Zhang and S. L. Braunstein. "continuous-variable gaussian analog of cluster states". Phys. Rev. A, 73:032318, Mar 2006. 